\title{
Incremental Scannerless Generalized LR Parsing
}

\author{
Master's Thesis
}

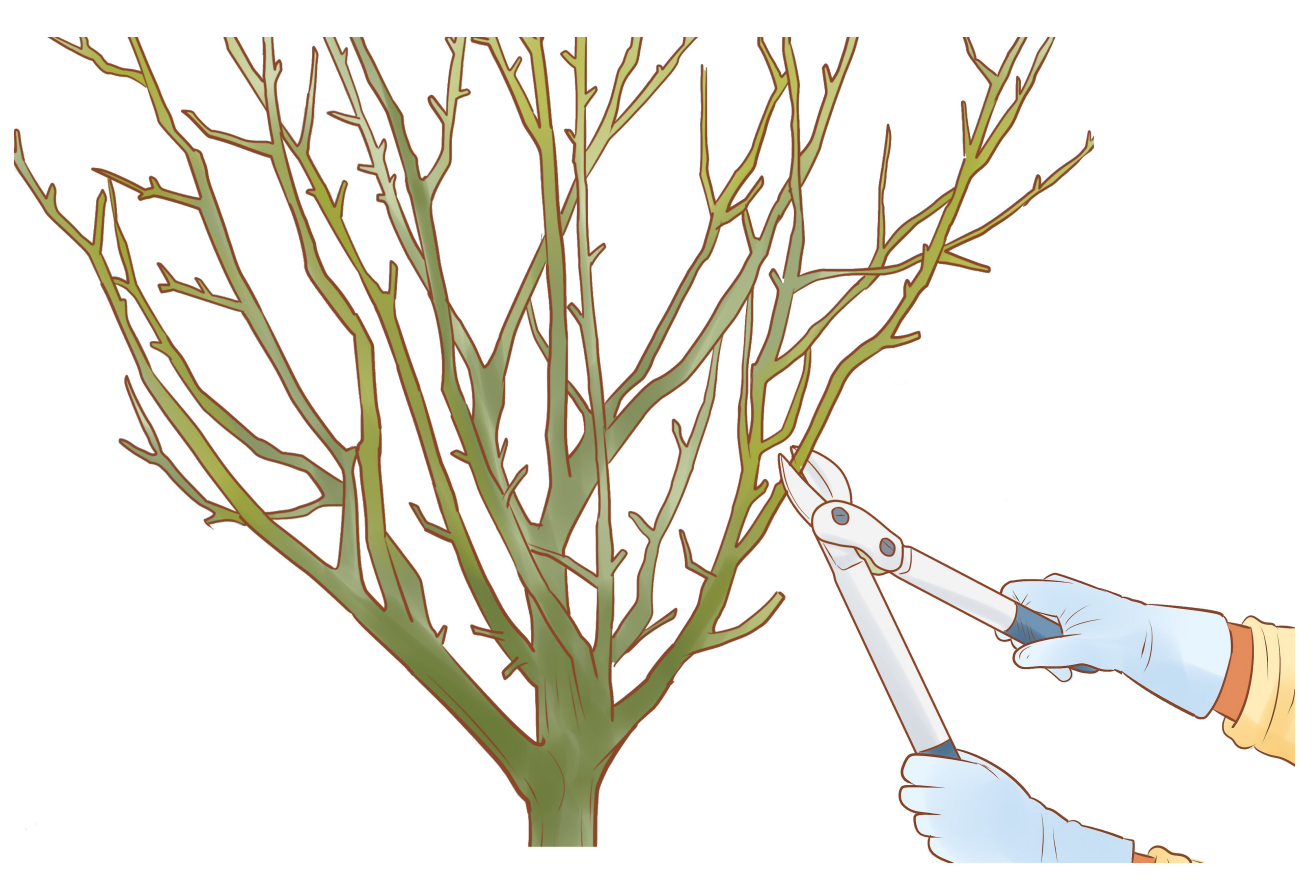

Maarten Peter Sijm 
This page is intentionally left blank. 


\section{Incremental Scannerless Generalized LR Parsing}

THESIS

submitted in partial fulfillment of the

requirements for the degree of

MASTER OF SCIENCE

in

COMPUTER SCIENCE

by

Maarten Peter Sijm

born in Honselersdijk, The Netherlands

To be defended publicly on

the 14th of July, 2021 at 10:00

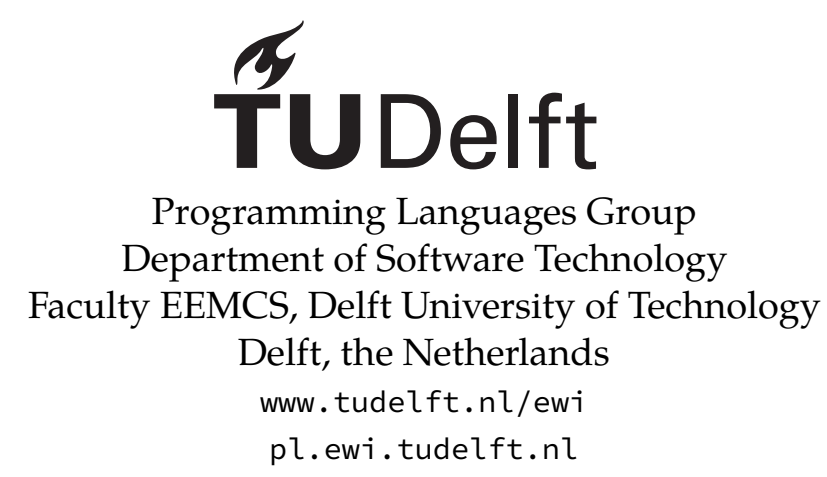


() 2021 Maarten Peter Sijm, CC-BY 4.0.

Cover picture: Pruning a tree.

Source: https: //www.wikihow.com/Prune-a-Fruit-Tree, CC BY-NC-SA 3.0.

All URLs have been archived at https://web. archive.org/ on 2021-07-07. 


\title{
Incremental Scannerless Generalized LR Parsing
}

\begin{abstract}
The Scannerless Generalized LR (SGLR) parsing algorithm supports the development of composed languages seamlessly but does not support incremental parsing. The Incremental Generalized LR (IGLR) parsing algorithm, on the other hand, does not support the seamless composition of languages. This thesis presents the Incremental Scannerless Generalized LR (ISGLR) parsing algorithm and investigates the effects of combining the SGLR and IGLR parsing algorithms. While the algorithmic differences are orthogonal, the fact that scannerless parsing relies on non-deterministic parsing for disambiguation has a negative impact on incrementality. Nonetheless, we show that the ISGLR parsing algorithm performs better than the batch SGLR parsing algorithm in typical scenarios. On average, the ISGLR parser can reuse $99 \%$ of a previous parse result. When parsing from scratch, the ISGLR parser has a $24 \%$ run time overhead compared to the SGLR parser, but when parsing incrementally for changes that are smaller than $1 \%$ of the input size on average, it has a $9 \times$ speedup.
\end{abstract}

\section{Keywords}

incremental, scannerless, parsing, GLR, IGLR, SGLR, ISGLR, imploding, syntax, Spoofax

\section{Thesis committee}

Chair:

Committee Member:

Committee Member:

Committee Member:

University Supervisor:
Prof. Dr. E. Visser,

Dr. C. Bach Poulsen,

Prof. Dr. T. van der Storm,

Prof. Emiritus P.D. Mosses,

J. Denkers MSc,
Faculty EEMCS, TU Delft Faculty EEMCS, TU Delft CWI / University of Groningen Swansea University / TU Delft Faculty EEMCS, TU Delft 
This page is intentionally left blank. 


\section{Preface}

This thesis marks the end of my seven years of studying at the TU Delft, of which the final two-and-a-half years I should have worked on my thesis. There have been many distractions for me during this final period, but they have also helped me through it, in one way or another. The list of people I want to thank is long, but I will make an attempt to mention most of them.

Firstly, I would like to thank Eelco Visser and Jasper Denkers for supervising me throughout my thesis. Our many meetings have helped me reflect on and sort out ideas for my research. The hallway conversations with other members of the Programming Languages (PL) group have also played a big part in this. I am thankful that Eelco suggested presenting my work at the SPLASH 2019 conference in Athens (see Appendix B), where I got to meet other researchers who were also interested in parsing and liked to discuss ideas. Also, thanks to the PL group for letting me use one of their servers to run the evaluation scripts (for which Danny and Daniël were a great help) and for providing helpful feedback on my enhancements to Spoofax (see Appendix C).

One of the larger "distractions" was being a teaching assistant (TA) for the Bachelor courses of Computer Science and Engineering at the TU Delft. I would like to thank the many people I have worked with, both my colleague TAs and the teachers. The lab sessions and grading sessions provided some welcome social interactions.

I would also like to thank my friends and rubber ducks ${ }^{1}$ for listening to my ramblings about parsing, providing suggestions for improvements and commenting on drafts of this thesis. My friends from the PL group, Bram, Chiel, Jeff, Luka, and Taico in particular, have provided some well-appreciated social distractions at the fourth floor and online. In addition, big thanks to Mathias and Mitchell, my buddies from the Westland with whom I have worked together since day one at the university, and hope to work with for many years to come at our startup company with Roy and Ruben.

I would be nowhere near where I am today without my supporting family, especially my parents and sister. Talking with them about working with parse trees has earned me the jocular title softwarehovenier ("software gardener"). And last, but definitely not least, my biggest thanks go out to my girlfriend Davina, who can make me calm even in the most stressful times.

Maarten Peter Sijm

Honselersdijk, the Netherlands

7 th July 2021

Disclaimer: no real trees were harmed during the making of this thesis.

\footnotetext{
${ }^{1}$ https://rubberduckdebugging.com/ or https://en.wikipedia.org/wiki/Rubber_duck_debugging
} 
This page is intentionally left blank. 


\section{Contents}

Preface

iii

Contents $\quad$ v

List of Figures $\quad$ vii

List of Tables $\quad$ ix

1 Introduction 1

2 Background 3

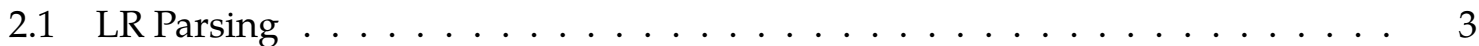

2.2 Generalized LR Parsing . . . . . . . . . . . . . . . . . . . . 6

2.3 Incremental Generalized LR Parsing . . . . . . . . . . . . . . . . . . 8

2.4 Scannerless Generalized LR Parsing . . . . . . . . . . . . . . . . . . . 10

3 Incremental Scannerless Generalized LR Parsing $\quad 17$

3.1 ISGLR Parsing by Example . . . . . . . . . . . . . . . . . . . . 17

3.2 Non-determinism in ISGLR Parsing . . . . . . . . . . . . . . . . . . . 24

3.3 Valid Parse Node Reuse . . . . . . . . . . . . . . . . . . . . . . . . . . . . 29

3.4 ISGLR Parsing Algorithm . . . . . . . . . . . . . . . . . . . . 31

3.5 Implementation in Modular Architecture . . . . . . . . . . . . . . . 36

4 Incremental Post-Processing 39

4.1 Imploding . . . . . . . . . . . . . . . . . . . . . . 39

4.2 Tokenization . . . . . . . . . . . . . . . . . . 43

5 Performance Evaluation $\quad 49$

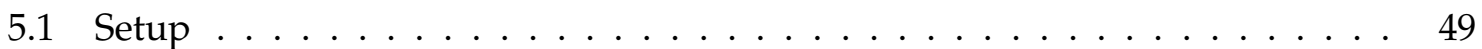

5.2 Measurements Results . . . . . . . . . . . . . . . . . . . . . . . 52

5.3 Time Benchmark Results . . . . . . . . . . . . . . . . . . . . . . 54

5.4 Memory Benchmark Results . . . . . . . . . . . . . . . . . . . . . 57

5.5 Threats to Validity . . . . . . . . . . . . . . . . . . . . . . 60

6 Related Work $\quad 61$

6.1 Incremental LR Parsers . . . . . . . . . . . . . . . . . . . . . . . 61

6.2 Incremental Recursive Descent Parsers . . . . . . . . . . . . . . . . . . . 63

6.3 Incremental Parser Combinators . . . . . . . . . . . . . . . . . . . . . 64 
7 Conclusion $\quad 65$

7.1 Future Work . . . . . . . . . . . . . . . . . . . . . . 65

$\begin{array}{lr}\text { Bibliography } & 67\end{array}$

$\begin{array}{ll}\text { Acronyms } & 71\end{array}$

$\begin{array}{ll}\text { Glossary } & 73\end{array}$

A Full Evaluation Results

A.1 Measurements . . . . . . . . . . . . . . . . . . . . 77

A.2 Time Benchmarks . . . . . . . . . . . . . . . . . . . . . . 92

B SPLASH Conference 2019 - ACM Student Research Competition 103

B.1 Extended Abstract . . . . . . . . . . . . . . . . . . . . . . . . . . 104

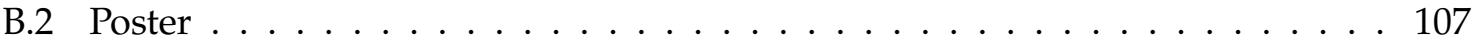

B.3 Grand Finals . . . . . . . . . . . . . . . . . . . . . . . . 108

$\begin{array}{ll}\text { C Spoofax Enhancements } & 113\end{array}$ 


\section{List of Figures}

2.1 A small expression grammar, $G_{\mathrm{Exp}}$, and its corresponding parse table. . . . . . 4

2.2 A parse tree for the input " $a+a^{\prime \prime}$, parsed according to the grammar $G_{\operatorname{Exp}}$ from Figure 2.1. . . . . . . . . . . . . . . . . . . .

2.3 The process of parsing the input " $a+a$ " according to the context-free grammar

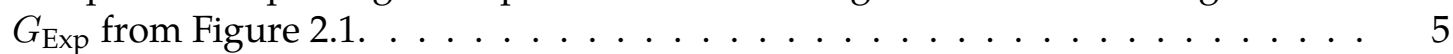

2.4 A part of the process of parsing " $a+a \times a^{\prime \prime}$ using $G_{\text {Exp }}$ from Figure 2.1. . . . . . 7

2.5 A screenshot of Harmonia, an IDE that uses the IGLR parsing algorithm (Wagner

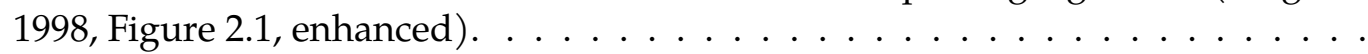

2.6 A snapshot of the state of an IGLR parser after a programmer has inserted the character ' $b$ ' in an existing identifier "acd". . . . . . . . . . . 9

2.7 A small expression grammar, specified using SDF3 syntax. . . . . . . . . . . . . . 11

2.8 The normalization of optionals and lists in SDF3 . . . . . . . . . . . . . . . 12

2.9 An SDF3 example that uses lexical disambiguation constructs. . . . . . . . . . . . . 13

2.10 The current parsing pipeline in the Spoofax IDE. . . . . . . . . . . . . . . . 13

3.1 The SDF3 definition of a small grammar. . . . . . . . . . . . . . . . . . . 18

3.2 The parse tree for input " $\mathrm{ab}=42 \star 42$ " according to the grammar in Figure 3.1(b). 20

3.3 The diff between version 1 and version $2 \ldots \ldots \ldots \ldots \ldots \ldots$

3.4 The parse stack (left) and the input stack (right) during an incremental parse. . 22

3.5 The resulting parse tree after first parsing " $a b=42 \star 42$ " and then incrementally

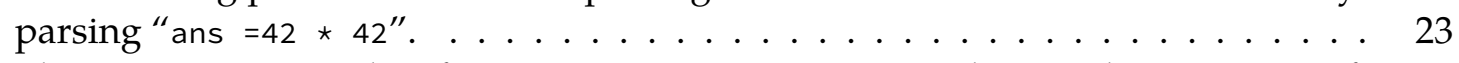

3.6 The active parse stacks after parsing " $a=x+y$ ", according to the grammar of Figure 3.1. . . . . . . . . . . . . . . . . 25

3.7 The active parse stacks at two points during the parsing of " $x=3 y=4 z=5$ ", according to the grammar of Figure 3.1. . . . . . . . . . . . . 27

3.8 Two production rules, added to the grammar of Figure 3.1, specified using SDF3

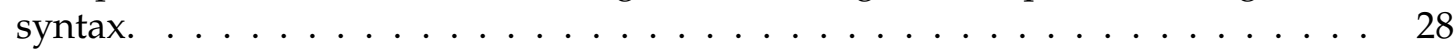

3.9 The active parse stacks at two points during the parsing of "return ab", according to the grammar of Figure 3.1, extended with the production rules of Figure 3.8. . $\quad 28$

3.10 A small expression grammar defined using kernel syntax. . . . . . . . . . . 30

3.11 The active parse stack and remaining input stack, after parsing " $x \star$ ", according to the grammar of Figure $3.10 \ldots \ldots \ldots \ldots$

3.12 The parsing pipeline in Spoofax, updated from Figure 2.10 for incremental pars-

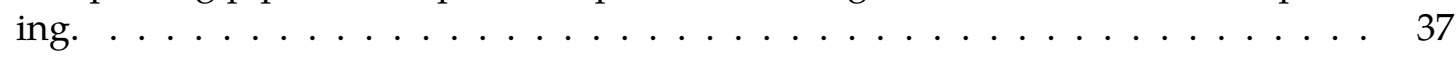

4.1 The imploded AST corresponding to the parse tree of Figure 3.2. . . . . . . . . 40

4.2 An example of the FlattenLists function of Algorithm 12. . . . . . . . . . . 42

4.3 The tokens that are generated from the parse tree of Figure 3.2. . . . . . . . . 43

4.4 An example token tree, generated from the parse tree of Figure 3.2 . . . . . . . . 45 
5.3 Parse times of the last 16 versions of several files and repositories from GitHub, excluding imploding and tokenization. . . . . . . . . . . . . 54

5.5 Parse times of the last 16 versions of several files and repositories from GitHub, including imploding and tokenization. . . . . . . . . . . . 56

5.7 Size of memory allocations during parsing for three variants of the JSGLR2 parser. 58

5.9 Size of the cache after parsing a file for three variants of the JSGLR2 parser. . . . 59

C.1 A screenshot of Spoofax in Eclipse, showing a language that uses Unicode characters. . . . . . . . . . . . . . . . . . . . 113

C.2 A screenshot of Spoofax in Eclipse, using a dark theme. . . . . . . . . . . . . 114 


\section{List of Tables}

5.1 Corpus used to evaluate the performance of the ISGLR parser. . . . . . . . . . 51

5.2 Measurements for parse nodes and breakdowns for the different languages in the evaluation corpus. . . . . . . . . . . . . . . . . . . . 52

5.4 Average parse times for the different languages in the evaluation corpus, excluding imploding and tokenization. . . . . . . . . . . . . . . 54

5.6 Average parse times for the different languages in the evaluation corpus, including imploding and tokenization. . . . . . . . . . . . . 56

5.8 Average memory allocations for the different languages in the evaluation corpus. 57

5.10 Average memory size of the cache for the different languages in the evaluation corpus. 
This page is intentionally left blank. 


\section{Chapter 1}

\section{Introduction}

The development of a new programming language follows a predefined set of stages. The language needs a parser, static semantics, and a translation to some other language or machine code to run programs written in the language. In addition, an advanced editor for a programming language has features like incremental parsing, syntax highlighting, refactoring actions, and identifier lookup. Developing all these features for a new programming language takes a lot of effort, and this makes experimenting with language development cumbersome.

Using a tool that generates a parser from a declarative grammar specification simplifies the first stage of developing a programming language. These generated parsers can be integrated into an editor, which then supports writing programs in the new language. One such tool is Syntax Definition Formalism 3 (SDF3) (Vollebregt, Kats, and Visser 2012; de Souza Amorim and Visser 2020), which generates parsers that make use of the Scannerless Generalized LR (SGLR) parsing algorithm by Visser (1997). While traditional parsers require both a lexing and a parsing phase, SGLR parsing merges these two phases by using grammars that are defined in terms of single characters. Any disambiguation that the lexer normally performs is encoded in the SDF3 grammar and is consequently handled by the SGLR parser. The main advantage of this parsing technique is that it reduces the complexity of composing grammars for different languages into a single grammar. This simplifies the development of languages that have a different language embedded in them, such as the markup language HTML, which has CSS and JavaScript embedded into the language.

However, SDF3 and the SGLR parsers it generates do not support incremental parsing. Wagner and Graham (1997b) created an Incremental Generalized LR (IGLR) parsing algorithm that aims to reuse as much as possible from a previous parse result when reparsing a changed file. The time it requires to reparse a file containing a textual change is proportional to the size of this change. Just like SGLR parsing, the IGLR parsing algorithm is based on the Generalized LR (GLR) parsing algorithm by Tomita (1985) and Rekers (1992). We provide the background on all these parsing techniques in Chapter 2.

In this thesis, we apply Wagner's incremental parsing technique to Visser's SGLR parsing algorithm. The different disambiguation methods of SGLR might interfere with incremental parsing in how much of the parse result can be reused. Therefore, in this thesis, we answer the following research question:

\section{What are the Effects of Combining Scannerless and Incremental GLR Parsing?}

To answer this research question, we have investigated the differences of both SGLR parsing and IGLR parsing compared to GLR parsing. In terms of their algorithms, these differences are orthogonal to each other and can be combined without difficulties. We present the resulting Incremental Scannerless Generalized LR (ISGLR) parsing algorithm in Chapter 3. While the algorithmic differences are orthogonal, we show that there exist non-trivial interactions 
between these two techniques: scannerless parsing introduces non-determinism in parsing that would not occur in non-scannerless parsing. This has a negative impact on the incrementality of the ISGLR parser, meaning that it can reuse less of the previous parse result than the non-scannerless IGLR parser.

To simplify the integration of the SGLR parser into an editor, its parse result is passed through two post-processing tasks: imploding removes irrelevant details from the parse result, and tokenization generates a list of tokens from the parse result. To our knowledge, these two tasks have not yet been described in the literature, so we provide their full description and algorithm in Chapter 4 before presenting an incremental algorithm for each post-processing task.

We have evaluated the performance of the ISGLR parsing algorithm using an automated benchmark suite that performs measurements and benchmarks on the ISGLR parser, based on a configuration file that describes the languages and sources used for the evaluation. In Chapter 5, we show that the ISGLR parser can reuse $99 \%$ of a previous parse result on average. We benchmark the run time performance using two scenarios: parsing files from scratch and parsing files incrementally. We show that ISGLR has a $24 \%$ run time overhead compared to SGLR when parsing from scratch, but when parsing incrementally for changes that are smaller than $1 \%$ of the input size on average, it has a $9 \times$ speedup.

We compare our approach with existing incremental parsing algorithms in Chapter 6. In Chapter 7, we conclude this work and state possibilities for future research.

Context We have developed the ISGLR parsing algorithm in the context of Spoofax (Kats and Visser 2010; MetaBorg 2016), a language workbench that simplifies the development of programming languages by generating many of the common features for a language based on declarative specifications. Besides generating an SGLR parser based on a grammar specification written in SDF3, it also generates a type checker based on a declarative specification of static semantics rules written in Statix (van Antwerpen et al. 2018), a compiler based on declarative transformation rules written in Stratego (Bravenboer et al. 2008), and an editor plugin that contains all of these features. Recent developments in Spoofax include incremental type checking (Aerts 2019) and incremental compilation (Smits, Konat, and Visser 2020), but it still lacks incremental parsing.

Contributions In summary, this thesis presents the following contributions.

- The Incremental Scannerless Generalized LR (ISGLR) parsing algorithm (Chapter 3).

- The negative impact of disambiguation in SGLR on incremental parsing (Chapter 3).

- A description and algorithm for imploding and tokenization (Chapter 4).

- Incremental algorithms for these two post-processing tasks (Chapter 4).

- An evaluation suite for measuring and benchmarking the performance of incremental parsers (Chapter 5).

- Insight into the performance of the ISGLR parser on representative inputs (Chapter 5). 


\section{Chapter 2}

\section{Background}

This chapter provides background for the Incremental Scannerless Generalized LR (ISGLR) parsing algorithm. Section 2.1 explains the Left-to-right Rightmost-derivation (LR) parsing algorithm by Knuth (1965) and Section 2.2 explains the Generalized LR (GLR) parsing algorithm by Tomita (1985) and Rekers (1992). Based on GLR parsing, Section 2.3 explains the Incremental Generalized LR (IGLR) parsing algorithm by Wagner and Graham (1997b) and Section 2.4 explains the Scannerless Generalized LR (SGLR) parsing algorithm by Visser (1997).

\subsection{LR Parsing}

The $\operatorname{LR}(k)$ parsing algorithm by Knuth (1965) is a bottom-up approach for parsing input streams into parse trees. This algorithm parses an input stream according to a given language, where any language can be specified using context-free grammars. Regarding context-free grammars, we will use the following terminology, adapted from Sipser (2012):

A context-free grammar is a 4-tuple $(N, \Sigma, R, S)$, where

1. $N$ is a finite set called the non-terminals;

2. $\Sigma$ is a finite set, disjoint from $N$, called the terminals;

- $N \cup \Sigma$ is called the set of symbols;

- $\$$ is a special terminal called the End-of-File (EOF) symbol, used to indicate the end of the input;

3. $R$ is a finite set of production rules, each of the form $A \rightarrow \alpha$ where $A$ is a non-terminal and $\alpha$ is a string of symbols; and

4. $S \in N$ is the start symbol.

\subsubsection{Lexical Analysis}

The input to the parser is a stream of text characters. Since the terminals of an LR grammar typically correspond to several characters in the text, a lexer will analyse the stream of text characters and transform it into a stream of tokens that can be used as terminals. The lexer will skip any layout characters, as they are just used as separation between tokens.

Depending on the lexical analysis system, language designers can define tokens using regular expressions (W. L. Johnson et al. 1968) or using an entirely different formalism. As an example, when they use YACC (S. C. Johnson 1975) to describe an LR grammar, they will usually define the tokens using Lex (Lesk and Schmidt 1975). 


\subsubsection{Parse Table Generation}

Before a context-free grammar can be used for LR parsing, a parse table generator generates a finite push-down automaton that will represent the LR parser. A two-dimensional parse table represents the possible state transitions for every state of the automaton, as shown in Figure 2.1. A parse table consists of two parts: an action table that maps state-terminal pairs to actions and a goto table that maps state-non-terminal pairs to other states.

In the action table, not all entries contain a valid action; trying to look up an empty entry will result in an error in the parser. In the bottom-left of the table in Figure 2.1(b), some entries contain multiple actions; these are called conflicts and will also result in an error.

$$
\begin{gathered}
G_{\operatorname{Exp}}= \\
(\{S, E\},\{a,+, \times\}, R, S)
\end{gathered}
$$

The set of rules, $R$, is:

$$
\begin{aligned}
& S \rightarrow E \\
& E \rightarrow E+E \\
& E \rightarrow E \times E \\
& E \rightarrow a
\end{aligned}
$$

\begin{tabular}{c||c|c|c|c||c|c}
\multicolumn{1}{c||}{ state } & \multicolumn{5}{c||}{ actions } & \multicolumn{2}{c|}{ gotos } \\
\cline { 2 - 5 } & + & $\times$ & $a$ & $\$$ & $S$ & $E$ \\
\hline 0 & & & $S(2)$ & & & 1 \\
1 & $S(3)$ & $S(4)$ & & Accept & & \\
2 & $R\left(E_{a}\right)$ & $R\left(E_{a}\right)$ & & $R\left(E_{a}\right)$ & & \\
3 & & & $S(2)$ & & & 5 \\
4 & & & $S(2)$ & & & 6 \\
5 & $S(3) / R\left(E_{+}\right)$ & $S(4) / R\left(E_{+}\right)$ & & $R\left(E_{+}\right)$ & & \\
6 & $S(3) / R\left(E_{\times}\right)$ & $S(4) / R\left(E_{\times}\right)$ & & $R\left(E_{\times}\right)$ & &
\end{tabular}

(b) The parse table of $G_{\text {Exp }}$. Shift actions are abbreviated to 'S' and Reduce (a) The formal definition of $G_{\text {Exp. }}$ actions to ' $R$ '. The actions will be explained in Section 2.1.3.

Figure 2.1: A small expression grammar, $G_{\mathrm{Exp}}$, and its corresponding parse table. The parse table is generated using http://jsmachines. sourceforge. net/machines/lr1.html.

\subsubsection{Parsing Algorithm}

The two inputs to the LR parsing algorithm are a parse table and an input stream of tokens. The output of the parser is a parse tree, an example of which is shown in Figure 2.2. In this tree, the leaf nodes correspond to the terminals of the grammar and the non-leaf nodes, also called parse nodes, correspond to the production rules.

During parsing, the parser maintains a parse stack. The parse stack stores references to states in the parse table, always having the start state at the bottom of the stack. The current state of the parser is always at the top of the parse stack. Each link between two stack nodes stores a parse node or terminal.

The parser uses the current state and the first terminal from the input stream to get an action from the action table, as shown in Figure 2.3. The three different types of actions decide how

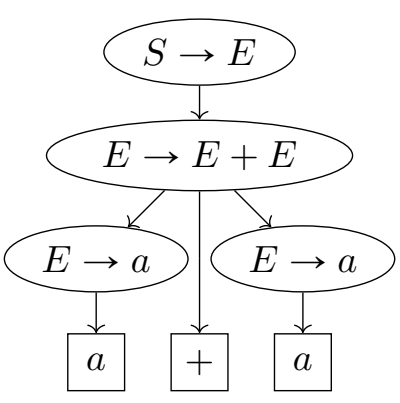

Figure 2.2: A parse tree for the input " $a+a^{\prime \prime}$, parsed according to the grammar $G_{\text {Exp }}$ from Figure 2.1. the parser should continue:

Shift( $s$ ) Consume a terminal $t$ from the input stream, push state $s$ onto the parse stack, and store $t$ on the created link to the previous stack node.

$\operatorname{Reduce}(A \rightarrow \alpha)$ Pop $|\alpha|$ items from the parse stack and create parse node $n$ corresponding to production rule $A \rightarrow \alpha$ with the popped nodes as children. Get state $s^{\prime}$ from the goto table using the state on top of the stack and non-terminal $A$, push state $s^{\prime}$ onto the parse stack, and store $n$ on the created link to the previous stack node.

Accept Finish parsing and return the parse tree that is stored on the link between the only two remaining nodes on the parse stack. 
Parse stack

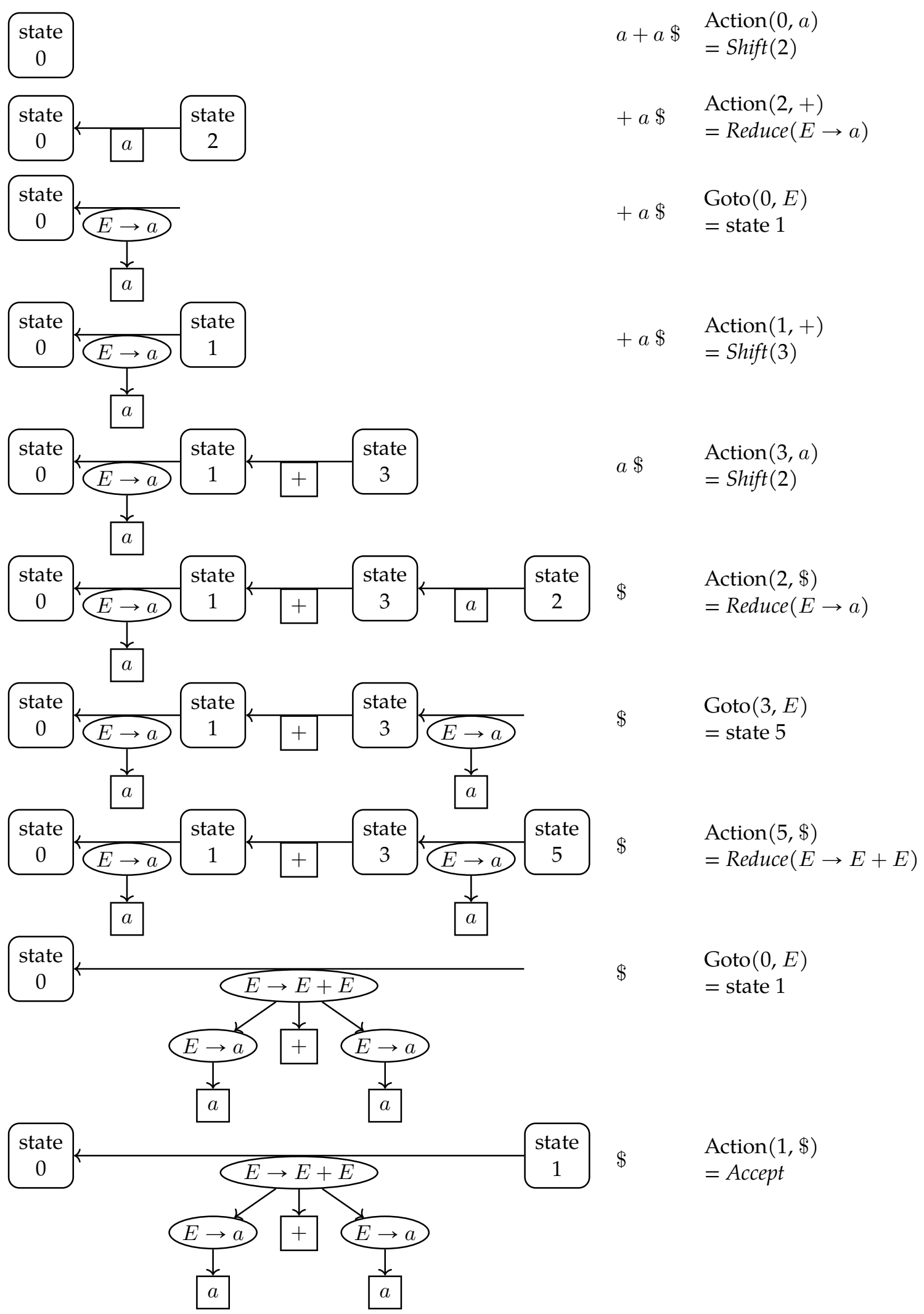

\section{Remaining input Action / Goto}

$$
\begin{array}{ll}
a+a \$ & \begin{array}{l}
\text { Action }(0, a) \\
=\operatorname{Shift}(2)
\end{array} \\
+a \$ & \begin{array}{l}
\text { Action }(2,+) \\
=\operatorname{Reduce}(E \rightarrow a)
\end{array} \\
& \\
+a \$ \quad \begin{array}{l}
\text { Goto }(0, E) \\
=\text { state } 1
\end{array}
\end{array}
$$

$\operatorname{Action}(1,+)$

$=\operatorname{Shift}(3)$

$\operatorname{Action}(2, \$)$

$=\operatorname{Reduce}(E \rightarrow a)$

$\operatorname{Goto}(3, E)$

$\operatorname{Action}(5, \$)$

$=\operatorname{Reduce}(E \rightarrow E+E)$

Goto $(0, E)$

Action $(1, \$)$

Accep

Figure 2.3: The process of parsing the input " $a+a^{\prime \prime}$ according to the context-free grammar $G_{\text {Exp }}$ from Figure 2.1. The EOF symbol $\$$ is appended to the input before parsing. In this figure, Reduce actions are processed in two steps: the first step shows popping items from the parse stack and the second step shows pushing a new state, determined via the goto table. 


\subsubsection{Bounded Versus Unbounded Lookahead}

The $k$ in "LR $(k)$ parsing" stands for the number of terminals that the parser is allowed to look ahead when determining the next action from the parse table. The parser that we covered so far only looks at the first terminal in the input stream to determine the next action, so it is an LR(1) parser. In general, we will assume that a parser has a lookahead of one terminal when $k$ is not specified.

All $\operatorname{LR}(k)$ parsers (with $k \geqslant 1$ ) are equally powerful because an $\operatorname{LR}(k)$ grammar can always be transformed into an LR(1) grammar (Mickunas, Lancaster, and Schneider 1976). However, $\operatorname{LR}(k)$ parsers cannot handle all context-free grammars. We say that a grammar requires a parser with unbounded lookahead if there exists no $\operatorname{LR}(k)$ parser that can parse all strings in the language of the grammar. For example, parsing $\mathrm{C}^{++}$requires unbounded lookahead: the statement int (a) , b , c , d , ...; can be interpreted either as a variable declaration list (the first being redundantly parenthesized) or as a comma-separated list of expressions (the first being a cast to int), depending on the final expression in the list. Since there can be arbitrarily many identifiers preceding this final expression, the parser theoretically needs infinite lookahead to disambiguate (Willink 2001, p. 147).

\subsection{Generalized LR Parsing}

Rekers (1992) created the GLR parsing algorithm based on the natural language parsing algorithm by Tomita (1985). Rekers' algorithm overcomes the limitation of LR parsing by simulating unbounded lookahead, so it can handle all context-free grammars.

GLR parsing makes use of existing LR parse table generators. Whereas parse table entries with multiple actions generated an error for plain LR parsing, GLR parsing will instead explore all possible actions. The parser will immediately apply any possible Reduce actions, while it saves up Shift actions for the different stacks and only applies them once it has processed all Reduce actions. Effectively, this means that all active parse stacks are synchronized at the same location in the input stream.

Rekers uses a Graph-Structured Stack (GSS) to represent the parse stacks. The active parse stacks each have a different top in the GSS. All stacks have the same root, represented by the start state, just like the linear parse stack used in LR parsing. Some examples of GSSs are shown in Figure 2.4. Besides the regular pushing to and popping from the stack, three other things can happen during GLR parsing:

Forking The parser forks new parse stacks from an existing one whenever it can execute more than one action. This happens when it encounters a conflict in the parse table, as shown in Figure 2.4(a). The parser also forks the parse stacks when a Reduce action can be applied over multiple paths in the GSS, as shown in Figure 2.4(d).

Merging The parser merges parse stacks back together when actions from different parse stacks result in the same state. Examples of this are shown in Figures 2.4(b) and 2.4(e).

Discarding The parser discards a parse stack when it has no available actions. The parser halts and returns an error when all parse stacks have been discarded.

If the GLR parser needs to merge parse stacks and does not discard the resulting stack until it finished parsing, an ambiguity remains in the final result. This ambiguity is represented in a parse node by storing multiple derivations in it. When a parse tree contains (or may contain) ambiguities, it is referred to as parse forest since multiple non-ambiguous parse trees could be constructed from it.

When a parser needs to fork the parse stack during parsing to achieve unbounded lookahead, we say that it is parsing non-deterministically. This does not imply that the final result contains ambiguities or that the grammar is ambiguous at all. 


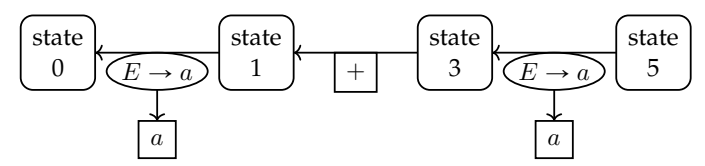

(a) The next terminal in the input stream is $\times$ and $\operatorname{Action}(5, \times)=\{\operatorname{Shift}(4), \operatorname{Reduce}(E \rightarrow E+E)\}$. The Reduce action is applied immediately using $\operatorname{Goto}(0, E)=$ state 1 and the parse stack forks. The Shift $(4)$ action for the original stack will be applied after the Reduce action has been processed.

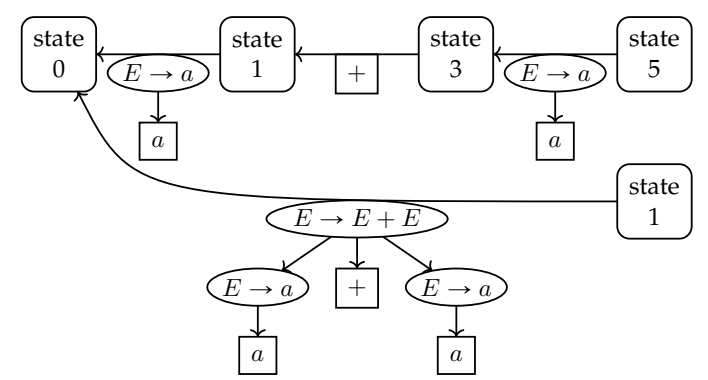

(b) For the new parse stack, Action $(1, \times)=$ Shift (4). The Shift actions for both stacks can now be applied. Since the new state is 4 in both cases, the stacks merge.

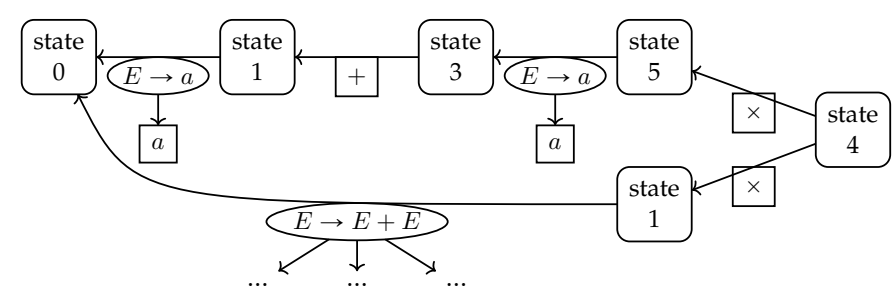

(c) After processing $\operatorname{Action}(4, a)=\operatorname{Shift}(2)$, Action $(2, \$)=\operatorname{Reduce}(E \rightarrow a)$, and $\operatorname{Goto}(4, E)=6$, the parse stack looks as follows:

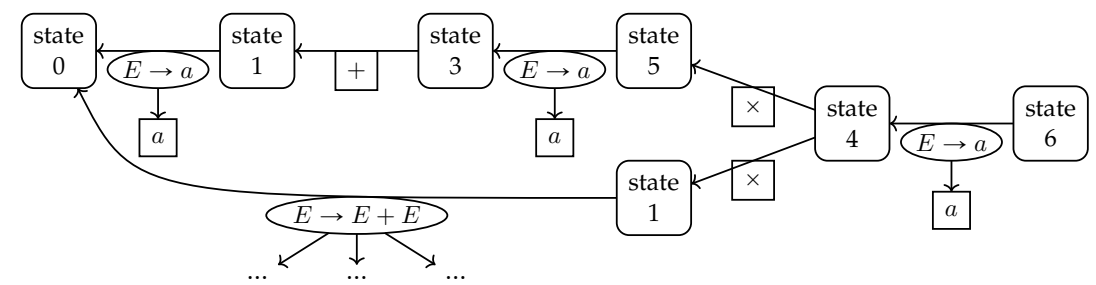

(d) With $\operatorname{Action}(6, \$)=\operatorname{Reduce}(E \rightarrow E \times E)$, there is only one possible action. However, there are two possible paths to reduce over: $3-5-4-6$ and $0-1-4-6$. Therefore, two new stacks are created using $\operatorname{Goto}(3, E)=$ state 5 and $\operatorname{Goto}(0, E)=$ state 1 .

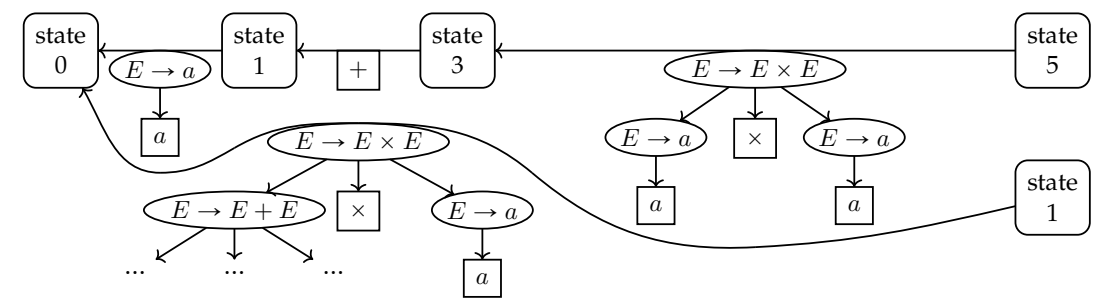

(e) The first stack has Action $(5, \$)=\operatorname{Reduce}(E \rightarrow E+E)$ left to process and with Goto $(0, E)=$ state 1 , the two stacks are merged again. The parse node on the only remaining edge will then contain two derivations. At this point, Action $(1, \$)=$ Accept and the parser returns this parse node. The second derivation is not shown, as it is symmetric with the first derivation. Note that if the Accept action would be executed first, the other stack would still be processed, to get both derivations.

Figure 2.4: A part of the process of parsing " $a+a \times a$ " using $G_{\text {Exp }}$ from Figure 2.1. 


\subsection{Incremental Generalized LR Parsing}

Wagner (1998) introduced the IGLR parsing algorithm, which extends the GLR parsing algorithm by Tomita (1985) and Rekers (1992). Boshernitsan (2001) applied this parsing algorithm in the interactive setting of an Integrated Development Environment (IDE) called Harmonia (a screenshot is shown in Figure 2.5). When a software developer that uses Harmonia makes a small change to a file that they are working with, the incremental parser only performs work for the changed regions of the input. The reduced parsing time greatly speeds up the development process.

We will call subsequent invocations of the parser in interactive settings incremental parses. On the other hand, we use the term batch parse for a single invocation of the parser on a file without reusing previous results.

Just like LR and GLR parsing, the IGLR parser has two phases: a lexer and a context-free parser.

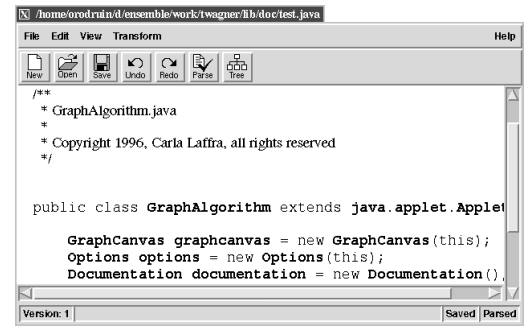

Figure 2.5: A screenshot of Harmonia, an IDE that uses the IGLR parsing algorithm (Wagner 1998, Figure 2.1, enhanced).

Incremental Lexical Analysis Wagner and Graham (1997a) describe an incremental lexer that maintains a mapping between the input character stream and the corresponding token stream. When the user changes the input, only the tokens nearby the changed regions need an updated lexical analysis to restore the mapping's consistency.

The incremental lexer updates the tokens directly in the leaf nodes of the previous version of the parse tree. Therefore, the time to find which tokens need to be updated is proportional to the height of the parse tree for every changed region of the input. In this process, all parse nodes that are ancestors of the changed token nodes can be marked as changed.

Incremental Parsing After the incremental lexer has updated the tokens in the leaf nodes of the parse tree, the IGLR parser by Wagner and Graham (1997b) performs a traversal along this tree, starting at the root node. For every parse node that the parser encounters, it checks whether it is unchanged and valid for reuse. If this is the case, it shifts this node onto the parse stack, determining the new state from the goto table using the non-terminal symbol on the left-hand side of the production rule of the shifted parse node. The parser then performs one of the following two movements: ${ }^{1}$

Descend If the parser can not reuse the current parse node, it moves to the first child node of the current parse node. In other words, the parser "breaks down" the current parse node to check its children.

Next If the parser can reuse the current parse node, it moves to the right sibling of the current parse node. If the current parse node is the last child in its parent, it moves to the right sibling of the closest ancestor that is not the last child in its parent.

Figure 2.6 shows an IGLR parser during such a traversal after a programmer has inserted the character ' $b$ ' in an existing identifier "acd" (Wagner 1998, Figure 6.1). It shows how the parser has already traversed the left part of the parse tree and has reused the parse nodes that have not changed, with the top of the parse stack (TOS) currently right before the changed token.

\footnotetext{
${ }^{1}$ Wagner (1998) named these movements leftBreakdown and popLookahead, respectively.
} 


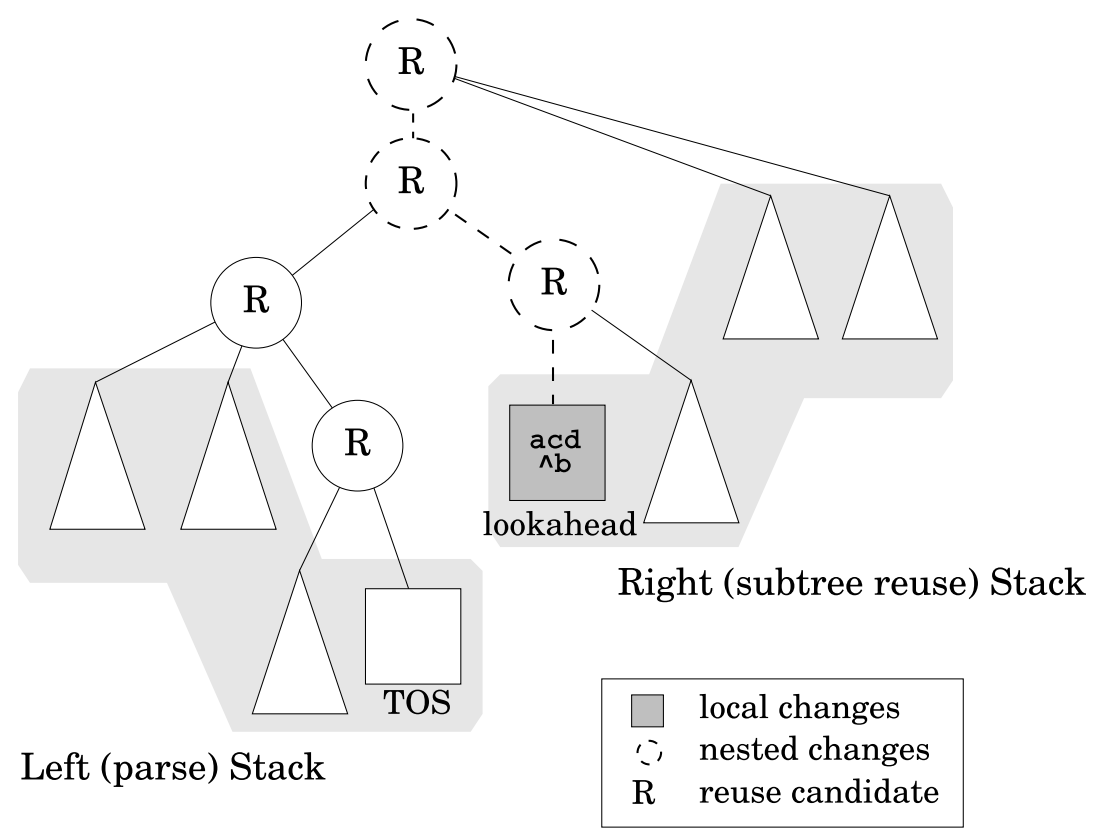

Figure 2.6: A snapshot of the state of an IGLR parser after a programmer has inserted the character ' $b$ ' in an existing identifier "acd" (Wagner 1998, Figure 6.1). The grey area on the left shows which parse nodes are on the parse stack right before the parser encounters the changed identifier, with "TOS" as the top of this stack. The grey area on the right shows which subtrees will be reused. This is not an explicit stack: the IGLR parser uses the Next movement to traverse over this part of the parse tree.

Valid Parse Node Reuse The IGLR parser can only reuse an unchanged parse node if the context surrounding this node allows this. To this end, it performs two tests, checking the context to the left and the context to the right. In other words, the parse node must be allowed to follow the part that has already been parsed, and the remaining input following this parse node must not have changed, respectively. We will give a more detailed example for both tests in Section 3.3 since the ISGLR parser uses the same tests.

Firstly, the IGLR parser uses a state matching test (Jalili and Gallier 1982) to check the context to the left, because the parse states capture this context by construction of the parse table. To implement this test, each parse node stores a reference to the state that was at the top of the parse stack before the node is pushed onto this stack. In a subsequent incremental parse, a parse node can only be reused if the state reference stored in the parse node is the same as the current parse state.

Secondly, the parser tests if the lookahead of the current parse node has changed, to check the context to the right. If this lookahead did change, the parser might need to take a different action from the parse table and cannot simply reuse this node. In a deterministic setting, only one ${ }^{2}$ token following the current parse node needs to be checked. However, during non-deterministic parsing, the number of lookahead tokens is unbounded, as explained in Section 2.2. In a subsequent incremental parse, there could be changes following a nondeterministic region that would cause a different parse stack to survive. Therefore, parse nodes that are created while multiple parse stacks are active, are marked as irreusable. Wagner and Graham (1997b) implement this by not storing a reference to a parse state in them. In this way, the state matching test will always fail for this parse node, which causes the parser to Descend from the node to check its children.

\footnotetext{
${ }^{2}$ The parser only needs to check one token ahead in the case of an $\operatorname{LR}(1)$ parser. An $\operatorname{LR}(k)$ parser would have to check the following $k$ tokens to see if they changed.
} 


\subsection{Scannerless Generalized LR Parsing}

Visser (1997) introduced the SGLR parsing algorithm, which is based on the scannerless variant of the Noncanonical Simple LR (NSLR) parsing algorithm by Salomon and Cormack (1989) combined with the GLR parsing algorithm by Tomita (1985) and Rekers (1992).

Unlike the other parsing approaches discussed so far, scannerless parsing uses only one parsing phase: it does not use a lexer. All elements of a language can be described using a single context-free grammar. This reduces the complexity of composing grammars for different languages into a single grammar, such as embedding a grammar for JavaScript or CSS into the grammar of HTML. Referencing symbols from another grammar is enough to merge one grammar into another. In contrast, composing grammars for a two-phase parser would also entail writing a lexer that produces tokens for both grammars. We will further compare language composition with non-scannerless parsers in Section 6.1.1.

Specification of Character-Level Grammars LR grammars are token-level grammars because they use tokens as the terminals of the context-free grammar. SGLR parsing is scannerless, meaning that it does not use a lexer, so it uses character-level grammars. Language designers can describe character-level grammars using characters as terminals. The resulting parse tree has character nodes instead of tokens as leaves. We will use the metalanguage Syntax Definition Formalism 3 (SDF3) (Vollebregt, Kats, and Visser 2012; de Souza Amorim and Visser 2020) to describe character-level grammars. See Figure 2.7(a) for an example grammar specified using SDF3. Grammars written in SDF3 can be converted to an LR parse table after a preprocessing step called grammar normalization, as described in Section 2.4.1.

Context-Free Versus Lexical Syntax SDF3 distinguishes between context-free syntax and lexical syntax sections of a grammar. The context-free syntax sections describe production rules that are analogous to the rules defined in a token-level grammar. The lexical syntax sections describe the syntax of lexical elements in the language, e.g., identifier names, numbers, strings, and operators. Language designers can describe the lexical elements of the grammar in a context-free way, just like the context-free part of the grammar. Constructs to disambiguate lexical elements will be discussed in Section 2.4.2.

Parser Implementation The ISGLR parser presented in Chapter 3 is implemented as an extension to the JSGLR2 implementation of the SGLR parsing algorithm. This implementation is written in Java and integrated into the Spoofax language workbench (Kats and Visser 2010; MetaBorg 2016). The JSGLR2 parser uses parse tables generated from SDF3 for parsing. The implementation of JSGLR2 is described in more detail in Section 2.4.3.

\subsubsection{Grammar Normalization}

A preprocessing step called grammar normalization converts the SDF3 specification to a minimal subset of SDF3 to simplify the parse table generator. Because of grammar normalization, SDF3 can have high-level features that do not need to be supported by the parse table generator, thus simplifying the grammar specification process for language designers. Using Figure 2.7, we will discuss some of the steps that grammar normalization executes.

Kernel Syntax Grammar normalization transforms all production rules in context-free syntax and lexical syntax sections to rules in a third type of section: kernel syntax. The kernel syntax section is denoted in SDF3 using the keyword syntax. In kernel syntax, the sort symbols (representing the non-terminals) are annotated with -CF and -LEX to remember the origin of the symbol. An injection is added for sorts used in both syntax sections, stating that the context-free sort is equal to the lexical sort. In the grammar of Figure 2.7, this happens for the Int sort, among others. 
context-free start-symbols

Exp

context-free syntax

Exp.Add = Exp "+" Exp

Exp.Num = Int

lexical syntax

LAYOUT $=[\backslash \backslash t \backslash n \backslash r]$

Int $=[0-9]+$

(a) The grammar, defined using highlevel SDF3.

(b) The same grammar, where grammar normalization transformed it to kernel syntax.

Figure 2.7: A small expression grammar, specified using SDF3 syntax. The grammar allows expressions (with the sort symbol Exp) to be either an addition or a number. Numbers can only be integers that consist of one or more numeric characters.

Character Classes The terminal symbols of an SDF3 grammar are character classes, which describe a set of characters in a compact notation. ${ }^{3}$ Grammar normalization will transform all character classes to use decimal Unicode values. For example, in the grammar of Figure 2.7(a), the Int sort is described by the character class [0-9], which gets normalized to $[\backslash 48-\backslash 57]$.

Literal Expansion The literals of the grammar, consisting of non-varying lexical elements like keywords and operators, are normalized to a list of character classes corresponding to the characters of the literal. In the example of Figure 2.7, the "+" literal that is used as an operator in the addition rule gets normalized to the rule $"+"=[\backslash 43]$. A literal keyword like "if" would get $" i f "=[\backslash 105][\backslash 102]$ as normalized production rule.

Layout Insertion A scannerless parser processes the input character by character. Therefore, the full grammar must also model the layout (whitespace characters) between grammar symbols. Manually adding this layout explicitly for every production rule would greatly decrease the readability of a grammar specification. Therefore, grammar normalization will insert optional layout symbols (denoted using "LAYOUT?-CF") between the symbols on the right-hand side of production rules that are defined in context-free syntax sections. For example, the addition rule (Exp. Add) gets explicit LAYOUT?-CF symbols around the "+" symbol in Figure 2.7(b).

\footnotetext{
${ }^{3}$ https://www.metaborg.org/en/latest/source/langdev/meta/lang/sdf3/reference.html\#character-classes
} 
Lists and Optionals SDF3 allows for three operators that indicate the allowed number of occurrences of a sort: ? is used for "zero or one" $^{\prime}$, is used for "zero or more", and + is used for "one or more". Each of these operators is normalized to two rules, as shown in Figure 2.8. When the list operators are used in a context-free syntax section, optional layout is also inserted between two sorts on the right-hand side of the normalized production rules.

Layout Production Rules To let the optional context-free layout symbol (LAYOUT?-CF) make use of the LAYOUT rule as defined in Figure 2.7(a), grammar normalization adds the production rules shown at the bottom of Figure 2.7(b). The normalization of LAYOUT?-CF is a combination of the optional and list normalizations as discussed in the previous paragraph. Also, the injection between the context-free and lexical is added here to make the final connection with the lexical definition of LAYOUT before normalization.

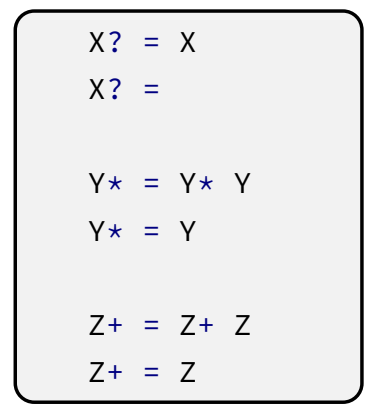

Figure 2.8: The normalization of optionals and lists in SDF3.

Start Symbol Grammar normalization adds two global start symbols to the normalized grammar. The first (<start $\rangle$ ) is used in the first production rule in Figure 2.7(b), which has the second start symbol (〈START>) and the EOF symbol (<EOF>) on the right-hand side. For the second start symbol, one rule is added for each start symbol defined in the start-symbols sections. Additionally, for start symbols defined in a context-free start-symbols section, this rule will also allow optional layout before and after the start symbol.

\subsubsection{Lexical Disambiguation Constructs}

Character-level grammars contain certain lexical ambiguities that do not occur in token-level grammars because these ambiguities are usually resolved by the lexer. In SDF3, reject rules and follow-restriction rules can be used to resolve two types of lexical ambiguities.

Reject Rules One type of lexical ambiguity that can occur, is that a string of tokens can be interpreted as different lexical elements that are all valid at that position. A typical example of this is that keywords (like if and return) can also be parsed as an identifier. To solve this, language designers using SDF3 can mark production rules with the keyword reject, causing the parser to reject these productions for the non-terminal on the left-hand side of the rule. Figure 2.9 shows an example of this.

Follow-Restriction Rules Another common ambiguity that arises in character-level grammars is that single identifiers could be split up into several identifiers, while there is no layout between those parts. To solve this, follow-restriction rules (denoted with -/-) can be used to prefer the longest match for lexical elements. Figure 2.9 shows an example with a followrestriction rule which states that an identifier cannot be followed by a letter because that would mean that this letter should be part of that identifier.

\subsubsection{Modular Parser Implementation}

The JSGLR2 parser in the Spoofax IDE implements the SGLR parsing algorithm in a modular way (Denkers 2018). This allows the implementation of extensions to the parser with as little code duplication as possible, increases the maintainability of the code, and allows composing variants if their modules do not have any overlapping changes.

The architecture of the JSGLR2 parser is modular in two dimensions. First, the parser can be configured to use variants of the runtime data structures for parsing. Second, the parsing algorithm is spread over modules that each contain an implementation of one or more of the subroutines of SGLR. We will refer to Denkers $(2018, \S 2.5)$ for the full SGLR algorithm. 
context-free syntax

Exp. Var $=$ Id

lexical syntax

$I d=[A-Z a-z]+$

Id = "IF" | "THEN" | "ELSE" | "ENDIF" \{reject

lexical restrictions

Id $-/-[A-Z a-z]$

Figure 2.9: An SDF3 example that uses lexical disambiguation constructs. The rule annotated with $\{$ reject $\}$ forbids that an identifier is equal to one of the strings in that rule. The followrestriction rule in the lexical restrictions section makes sure that an identifier cannot be followed by another letter.

Runtime Data Structures The JSGLR2 parser can use variants of the runtime data structures as described below. Constructing a variant of the parser requires passing factories for these data structures to the constructor of the Parser class. These factories will then instantiate the data structures during a parse.

ParseForest, ParseNode, Derivation, and CharacterNode are the data structures that make up the parse forest that the parser produces for a successful parse. Both ParseNode and CharacterNode are implementations of ParseForest. A ParseNode stores a list of Derivations, each of which stores a list of ParseForests again. A Derivation also stores for which production rule it was created. A CharacterNode stores which character it represents.

StackNode and StackLink make up the parse stack. A stackNode stores a state reference and a list of outgoing StackLinks. A StackLink stores references to the two StackNodes that it connects between, a ParseForest, and whether it is marked as rejected.

Parsestate stores the global variables used during parsing: active-stacks, accepting-stack, for-actor, for-actor-delayed, for-shifter, and the input stream.

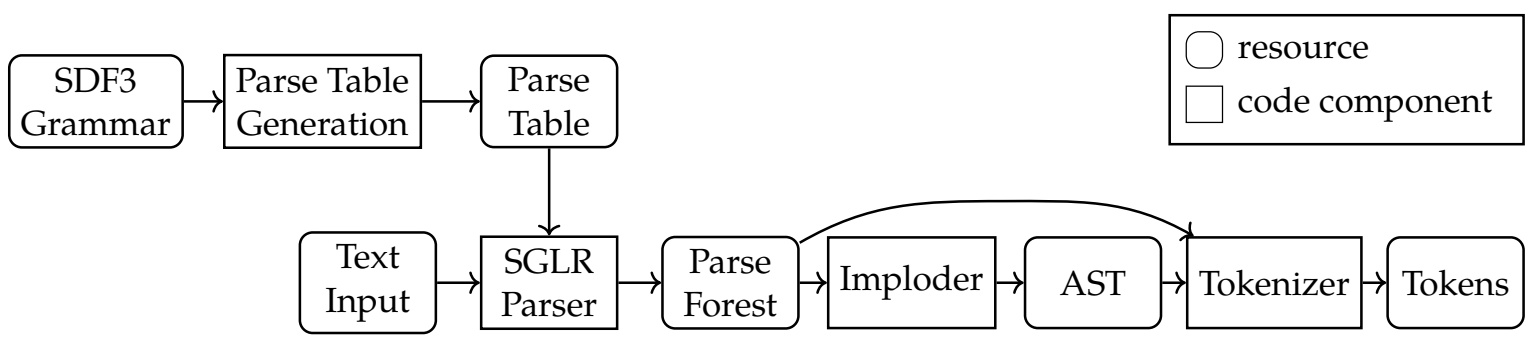

Figure 2.10: The current parsing pipeline in the Spoofax IDE. The top row of the pipeline is executed during language development, while the bottom row is executed every time a file is parsed. 
Parser Modules The Java class JSGLR2Implementation is the entry point to the complete parsing pipeline. It ties the SGLR parsing algorithm, the imploder, and the tokenizer together. An overview of the Java classes that implement parts of the parsing pipeline is given below. Additionally, Figure 2.10 shows a schematic overview of the complete parsing pipeline from input to output. Note that the parse table generator in the top row of this figure is implemented in the SDF module in Spoofax, i.e., it is not a part of JSGLR2.

Parser contains the main parse loop, deferring several parts of the algorithm to other components. It has methods for the following procedures of the SGLR algorithm: PARSE, calls PARSeCharacter for all characters in the input stream;

ParseCharacter, calls Actor for every active parse stack and calls Shifter after that; AстоR, fetches an action from the parse table and acts upon it; and

SHIFTER, pushes the current character onto all remaining active stacks.

Reducemanager has methods for the DoReductions and DoLimitedReductions procedures, which calculate the possible reduction paths in the GSS and call ReDUCER for every valid path. It also has a method that covers a large part of the REDUCER procedure. This method determines which branch of the Reducer to call, depending on other active parse stacks and their links to other stacks.

Reducer contains the code for the three branches of the REDUCER procedure: the case where both the new stack node and stack link already exist, the case where only the new stack node already exists, and the case where neither already exist.

StackManager handles the creation of stack nodes and stack links.

ParseForestManager handles the creation of parse nodes and derivations, and adding derivations to parse nodes.

Imploder transforms a parse forest to an Abstract Syntax Tree (AST). More details will be given in Section 4.1.

Tokenizer transforms the parse forest to a list of tokens and attaches these tokens to the AST. More details will be given in Section 4.2.

Parser Variants The current implementation of JSGLR2 supports the following parser variants, which have been implemented as modular extensions to the standard parser:

Elkhound McPeak and Necula (2004) introduced Elkhound parsing, an optimization to GLR parsers where it can fall back to the more efficient LR parsing algorithm when there is only one active parse stack. Denkers $(2018, \S 5.2)$ describes the implementation of Elkhound in the JSGLR2 parser.

Optimized Parse Forest Denkers (2018) introduced a variant of the JSGLR2 parser that skips the creation of parse nodes when they are not needed for the final AST. This includes parse nodes that would be created for lexical production rules and reject rules.

Data-Dependent de Souza Amorim, Steindorfer, and Visser (2018) extend SDF3 by allowing symbols to be parameterized by data and allow arbitrary computation at parse time. They use this extension to solve deep priority conflicts, which are priority conflicts between parse nodes that are not directly connected, but instead are separated by arbitrarily many levels of parse nodes, so regular priority declarations cannot be used to resolve these conflicts. 
Layout-Sensitive de Souza Amorim, Steindorfer, Erdweg, et al. (2018) extend SDF3 with layout constraint annotations, which allows parsing languages that are sensitive to layout. For example, Python uses indentation to indicate nesting in blocks of code, requiring this layout-sensitive parsing approach.

Error Recovery de Jonge et al. (2012) add error recovery to SGLR and implemented this in JSGLR1, the previous version of the SGLR implementation in Spoofax. The implementation of this extension in JSGLR2 is a work in progress.

We implemented the ISGLR parser presented in Chapter 3 in the modular architecture of JSGLR2. Details of the implementation in this architecture will be given in Section 3.5. 
This page is intentionally left blank. 


\section{Chapter 3}

\section{Incremental Scannerless Generalized LR Parsing}

This chapter presents and analyses the Incremental Scannerless Generalized LR (ISGLR) parsing algorithm to answer the research question stated in Chapter 1 . This parsing algorithm combines Scannerless Generalized LR (SGLR) parsing and Incremental Generalized LR (IGLR) parsing. The differences of these two parsing algorithms with respect to GLR parsing are orthogonal to each other and are combined without difficulties. While the algorithmic differences are orthogonal, we show that there exist non-trivial interactions between these two techniques.

The ISGLR parsing algorithm works as follows. First, it calculates a list of changes (called a diff) between the old and the new version of the input string. Then, starting at the root node, the parser tests whether parse nodes can be reused in the current context, and if not, breaks them down and continues with their children. The parser will always break down parse nodes that contain any of the changed positions as calculated by the diff. When the parser encounters irreusable parse nodes, which were created while the parser was parsing non-deterministically (see Section 2.3), it will break those down as well.

In Section 3.1, we apply the ISGLR parsing algorithm to an example to show how it works in practice. We show that increased non-determinism caused by scannerless parsing results in less reuse during an incremental parse using more examples in Section 3.2. The ISGLR parsing algorithm uses the same state matching test and lookahead test as Wagner and Graham (1997b) (see Section 2.3) to test whether reusing a parse node is valid, as we show in Section 3.3.

We write out the entire parsing algorithm in detail in Section 3.4. In particular, we highlight the changes required to the SGLR parser algorithm by Visser (1997). In Section 3.5, we describe the required changes to the JSGLR2 implementation by Denkers (2018), as described in Section 2.4.3.

\subsection{ISGLR Parsing by Example}

In this section, we will give an intuition of the ISGLR parsing algorithm using an example. The example is based on a small grammar that allows a list of variable assignment statements of the shape Stmt.Assign = ID "=" Exp. The allowed expressions are additions, multiplications, numbers and variables. The full SDF3 grammar is shown in Figure 3.1(a) and the normalized grammar in Figure 3.1(b).

In this example, we will consider the incremental parse of the input string "ab $=42$ * 42 " followed by "ans $=42 * 42$ " (hereafter called version 1 and version 2 , respectively). The parse tree of the first input string is shown in Figure 3.2. 


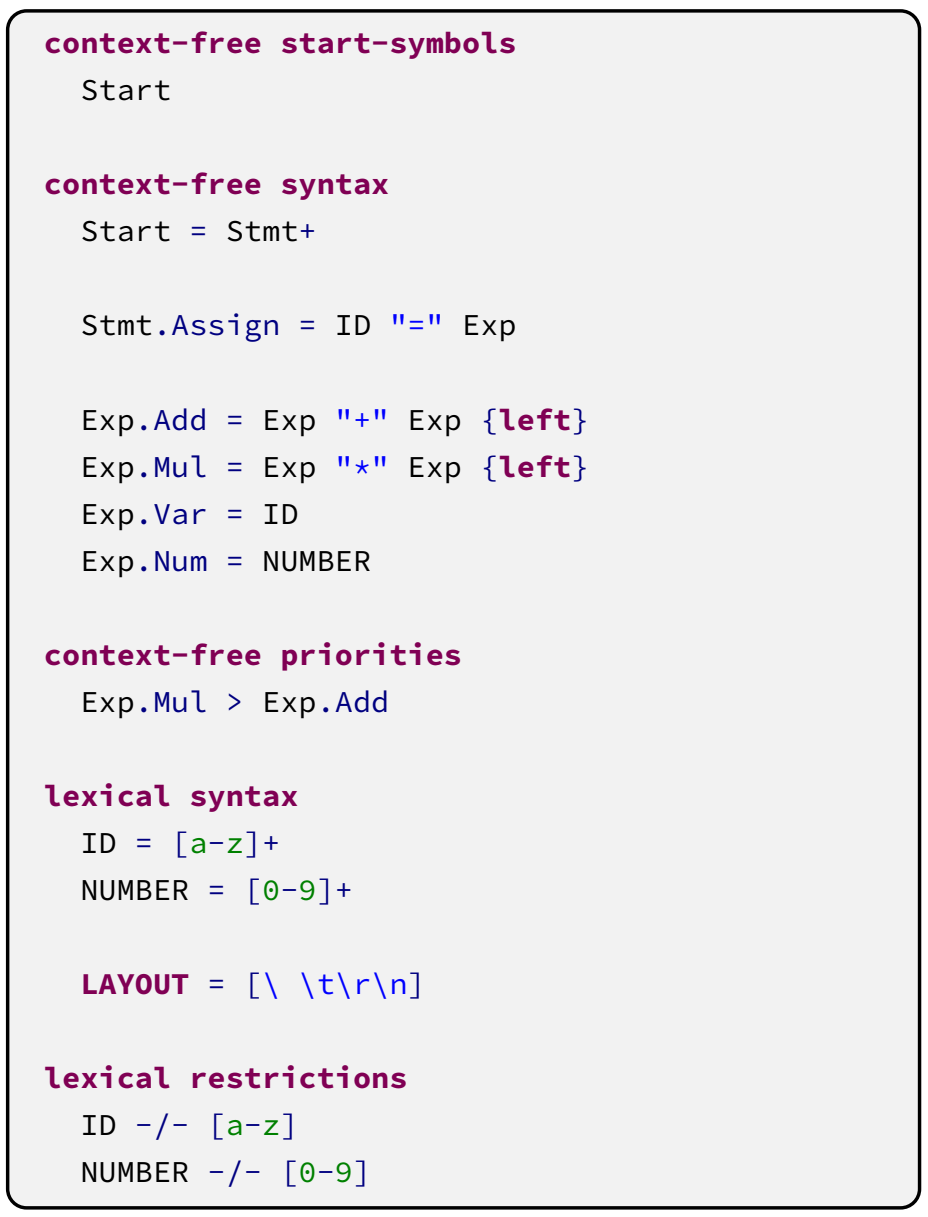

(a) The grammar, defined using high-level SDF3.

Figure 3.1: The SDF3 definition of a small grammar that allows a list of statements that assign an expression to a variable. The allowed expressions are additions, multiplications, numbers, and variables. 


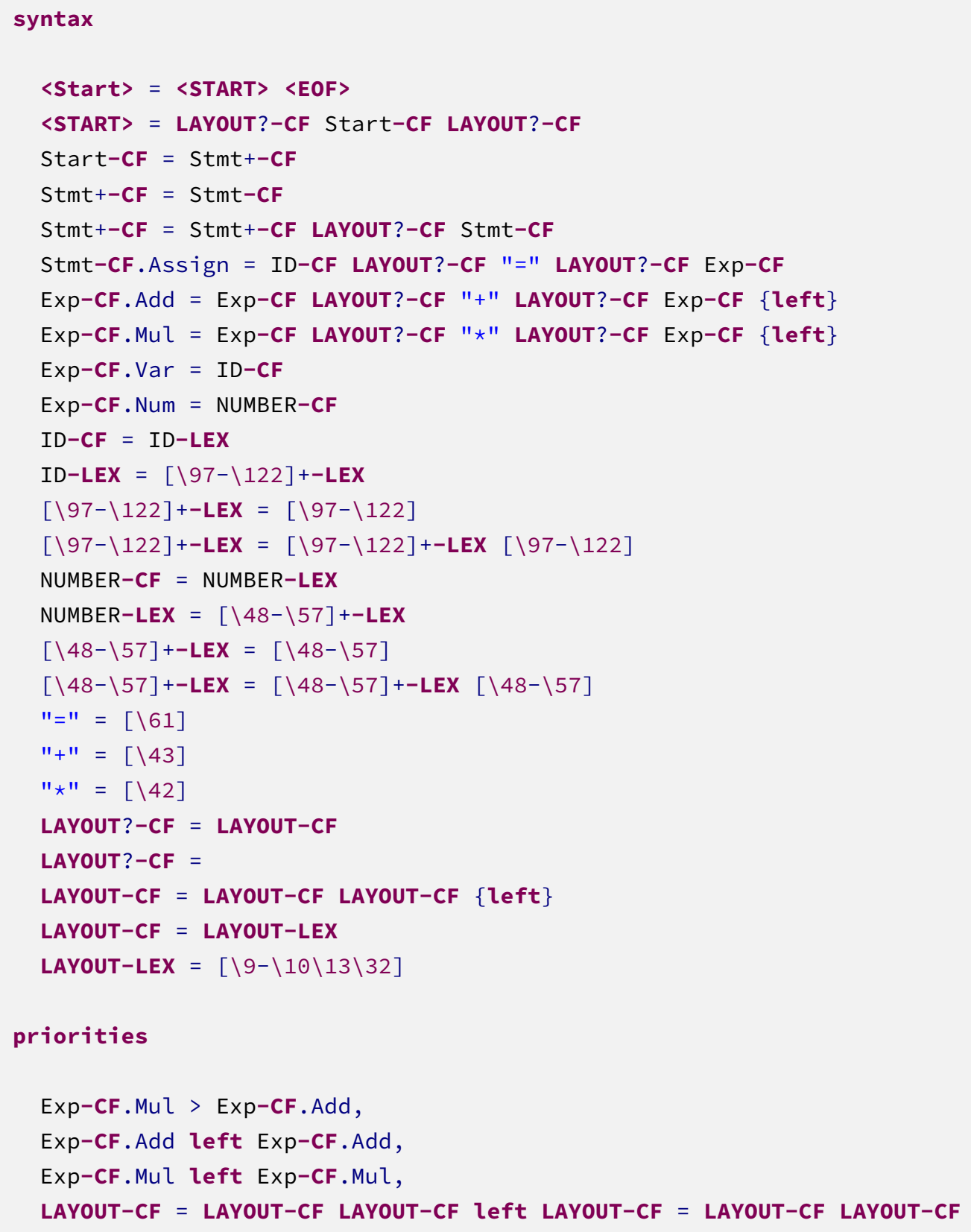

(b) The SDF3 grammar of Figure 3.1(a), normalized to kernel syntax.

Figure 3.1 (Continued) 


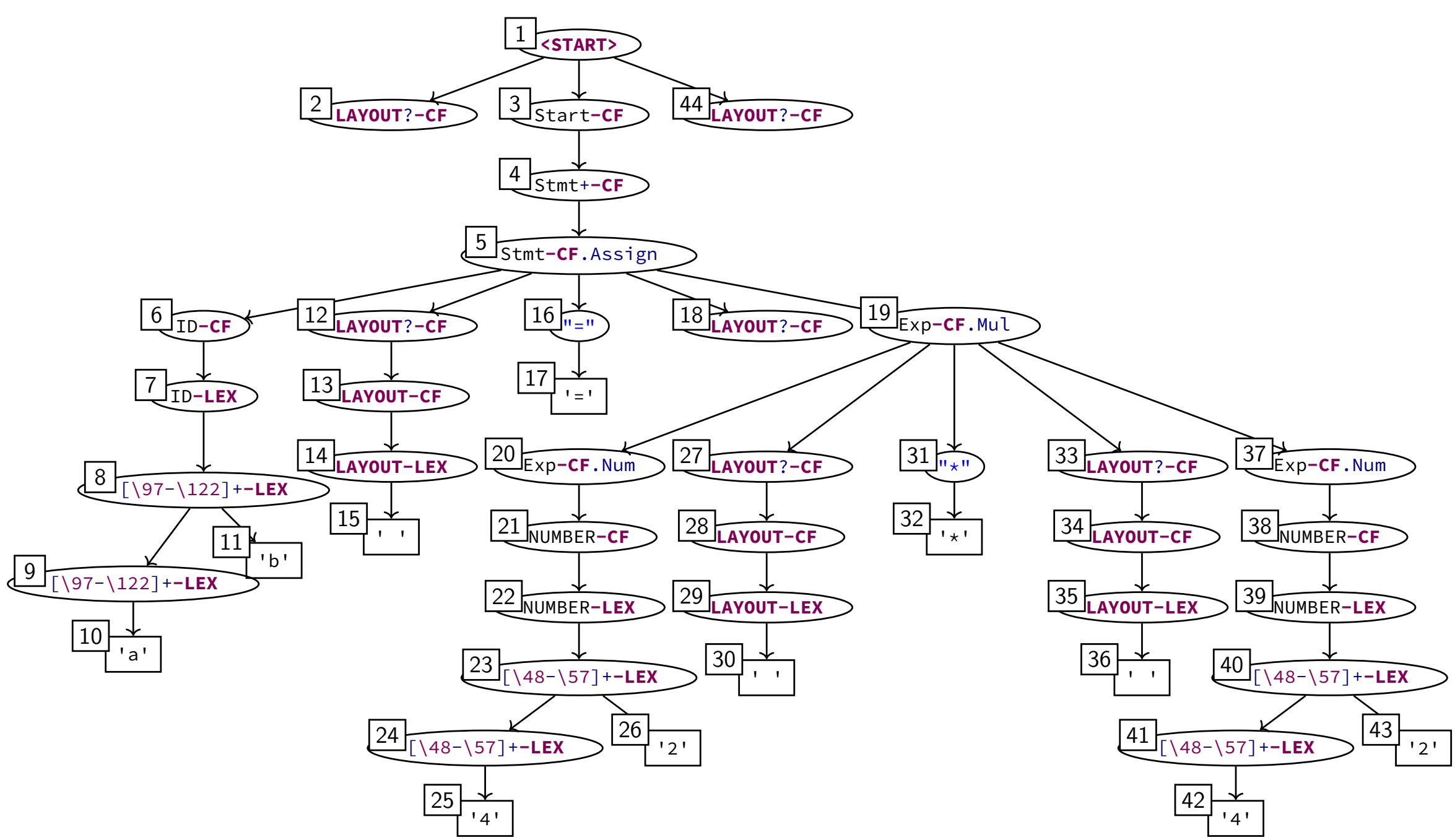

Figure 3.2: The parse tree for the input " $a b=42 \star 42$ " according to the grammar in Figure 3.1(b). 


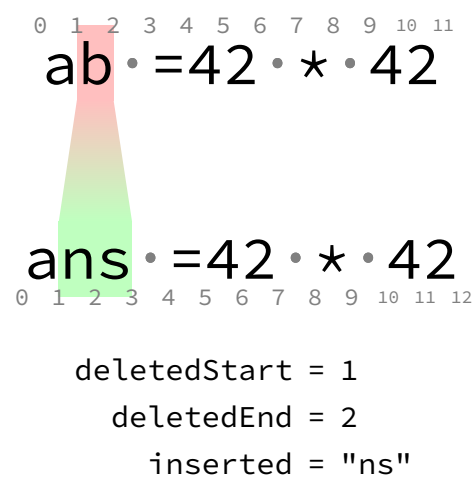

Figure 3.3: The diff between version 1 and version 2 .

Calculate Updates Using Diff As a first step, we compute the character-by-character difference between the two versions of the input string, using an existing diff algorithm. ${ }^{1}$ This algorithm gives a list of changes as a result. Calculating the diff between version 1 and version 2 yields a single change, as shown in Figure 3.3. This change indicates that the second character (the " $b$ ") is deleted and replaced with " $n$ s".

In general, each text change is modelled using three values: the start and end positions of the deleted text, and the string that is inserted at the start position. This models text replacements, insertions, and deletions. For insertions, the start and end index are equal, because for this change no text is deleted. For deletions, the string that is inserted is left empty.

When the calculated diff contains changes that have different lengths for the deleted and inserted text, the positions in subsequent changes become outdated. We will abstract over this detail since it only requires simple arithmetic to update these positions. In the remainder of this chapter, we assume that the parser can always correctly detect whether a certain position contains a change, based on this abstraction.

Incremental Input Stack The ISGLR parser uses an incremental input stack to consume input from. Whereas a regular input stream would store only characters, the incremental input stack can also store entire parse nodes. When the parse node at the top of the stack cannot be reused for whatever reason, it will be broken down and its children will be pushed back onto the stack in reverse order so that the first child ends up on top of the stack. The main reason for having to break down a parse node is when it contains a change from the calculated diff. Eventually, all parse nodes along the spine between the root node and the changes will be broken down. Other reasons for breaking down parse nodes will be discussed in the remainder of this chapter.

The approach of using an input stack differs from the IGLR parsing algorithm of Wagner and Graham (1998), which performs a walk along the parse tree after updating the tokens in the leaf nodes, as explained in Section 2.3. This requires the parse nodes to maintain a reference to their parent, which needs to be updated if a subtree is reused in a different node. Since we treat parse nodes as immutable, we use the incremental input stack to keep track of which parse nodes still need to be processed.

\footnotetext{
${ }^{1}$ In particular, the diff algorithm implemented in JGit: https : / / download . eclipse . org/jgit/ site / 5 . 6 . ๑ . 201912101111-r/apidocs/org/eclipse/jgit/diff/package-summary.html
} 
Incremental Parsing Now, we start parsing version 2. In Figure 3.4, we will follow the contents of the incremental input stack and the parse stack during parsing. The incremental input stack is initialized with the root of the parse tree of Figure 3.2 (node 1 ) and an EOF marker \$.

Node 1 contains the change that was calculated by the diff, so the parser breaks it down and pushes its children $(2,3$, and 44 ) onto the input stack. The parser then shifts node 2 onto the parse stack, since its symbol (LAYOUT?-CF) is in the goto table of the start state.

Following this, the parser has to break down multiple parse nodes successively, all the way until character node $a(10)$ is on top of the input stack. This node can then be shifted onto the parse stack. Note that its parent node 9 is not part of the change, but it is directly followed by a change, so it should still be broken down (more on this in Section 3.3.2).

Now that character node $b$ is at the top of the input stack, the update can be applied. Node $b$ is removed from the stack, while two new character nodes $n$ and $s$ are pushed back onto it.

With character node $n$ at the top of the input stack, the parser can reduce parse node $a$, creating node 47 with sort [\97-\122]+-LEx. On top of this new node, the parser shifts the $n$ onto the parse stack.

The parser then reduces the two nodes at the top of the parse stack into node 48 , which is another parse node with sort [\97-\122]+-LEX. After shifting the $s$ node, it performs the same reduction, creating node 49 .

Then, the parser performs two reductions, from parse node 49 to ID-LEX and from that node to ID-CF. Note that this is a node with the same sort as node 6, which was the first child of node 5 (with sort Stmt-CF. Assign) in the parse tree of the first version. Therefore, the parser can shift all other children of node 5 from the input stack onto the parse stack and reduce them all to a new parse node Stmt-CF.Assign.

Finally, the parser performs two more reductions, shifts parse node 44, and reduces the entire parse stack to the <START> symbol. With only the \$ node left in the input stack, the parser accepts the input. Figure 3.5 shows the resulting parse tree.

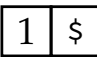

\begin{tabular}{|l|l|l|l|}
\hline 2 & 3 & 44 & $\$$ \\
\hline
\end{tabular}
2

2

2

2

2

2

2

2

\begin{tabular}{|l|l|}
\hline 2 & $a$ \\
\hline
\end{tabular}

\begin{tabular}{|l|l|}
\hline 2 & $\mathrm{a}$ \\
\hline
\end{tabular}

\begin{tabular}{|l|l|}
\hline 2 & 47 \\
\hline
\end{tabular}

\begin{tabular}{|l|l|l|}
\hline 2 & 47 & $n$ \\
\hline
\end{tabular}

\begin{tabular}{|l|l|}
\hline 2 & 48 \\
\hline
\end{tabular}

\begin{tabular}{|l|l|l|}
\hline 2 & 48 & $\mathrm{~s}$ \\
\hline
\end{tabular}

2049

\begin{tabular}{|l|l|}
\hline 2 & ID-LEX \\
\hline
\end{tabular}

\begin{tabular}{|l|l|}
\hline 2 & ID-CF \\
\hline
\end{tabular}

\begin{tabular}{|l|l|l|}
\hline 2 & ID-CF & 12 \\
\hline
\end{tabular} \begin{tabular}{|l|l|l|}
\hline 3 & 44 & $\$$ \\
\hline
\end{tabular}

\begin{tabular}{|l|l|l|}
\hline 4 & 44 & $\$$ \\
\hline
\end{tabular}

\begin{tabular}{|l|l|l|}
\hline 5 & 44 & $\$$ \\
\hline
\end{tabular}

\begin{tabular}{|l|l|l|l|l|l|l|}
\hline 6 & 12 & 16 & 18 & 19 & 44 & $\$$ \\
\hline
\end{tabular}

\begin{tabular}{|l|l|l|l|l|l|l|}
\hline 7 & 12 & 16 & 18 & 19 & 44 & $\$$ \\
\hline
\end{tabular}

\begin{tabular}{|l|l|l|l|l|l|l|}
\hline 8 & 12 & 16 & 18 & 19 & 44 & \$ \\
\hline
\end{tabular}

\begin{tabular}{|l|l|l|l|l|l|l|l|}
\hline 9 & $\mathrm{~b}$ & 12 & 16 & 18 & 19 & 44 & \$ \\
\hline
\end{tabular}

\begin{tabular}{|l|l|l|l|l|l|l|l|}
\hline $\mathrm{a}$ & $\mathrm{b}$ & 12 & 16 & 18 & 19 & 44 & \$ \\
\hline
\end{tabular}

\begin{tabular}{|l|l|l|l|l|l|l|}
\hline $\mathrm{b}$ & 12 & 16 & 18 & 19 & 44 & \$ \\
\hline
\end{tabular}

\begin{tabular}{|l|l|l|l|l|l|l|l|}
\hline $\mathrm{n}$ & $\mathrm{s}$ & 12 & 16 & 18 & 19 & 44 & \$ \\
\hline
\end{tabular}

\begin{tabular}{|l|l|l|l|l|l|l|l|}
\hline $\mathrm{n}$ & $\mathrm{s}$ & 12 & 16 & 18 & 19 & 44 & $\$$ \\
\hline
\end{tabular}

\begin{tabular}{|l|l|l|l|l|l|l|}
\hline s & 12 & 16 & 18 & 19 & 44 & \$ \\
\hline
\end{tabular}

\begin{tabular}{|l|l|l|l|l|l|l|}
\hline $\mathrm{s}$ & 12 & 16 & 18 & 19 & 44 & \$ \\
\hline
\end{tabular}

\begin{tabular}{|l|l|l|l|l|l|}
\hline 12 & 16 & 18 & 19 & 44 & $\$$ \\
\hline
\end{tabular}

\begin{tabular}{|l|l|l|l|l|l|}
\hline 12 & 16 & 18 & 19 & 44 & $\$$ \\
\hline
\end{tabular}

\begin{tabular}{|l|l|l|l|l|l|}
\hline 12 & 16 & 18 & 19 & 44 & $\$$ \\
\hline
\end{tabular}

\begin{tabular}{|l|l|l|l|l|l|}
\hline 12 & 16 & 18 & 19 & 44 & $\$$ \\
\hline
\end{tabular}

\begin{tabular}{|l|l|l|l|l|}
\hline 16 & 18 & 19 & 44 & $\$$ \\
\hline
\end{tabular}

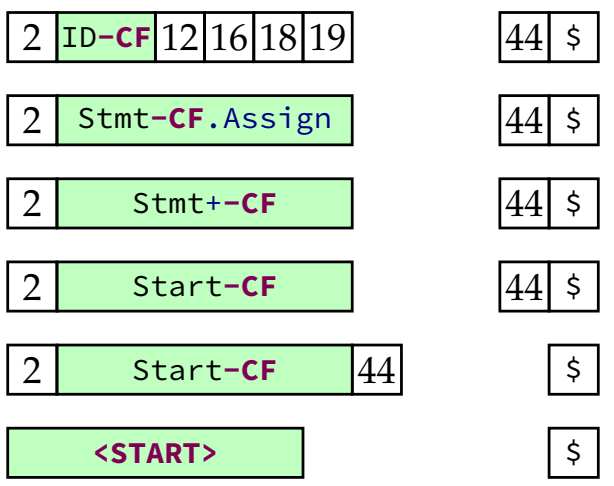

Figure 3.4: The parse stack (left) and the input stack (right) during an incremental parse, using the change from Figure 3.3. 


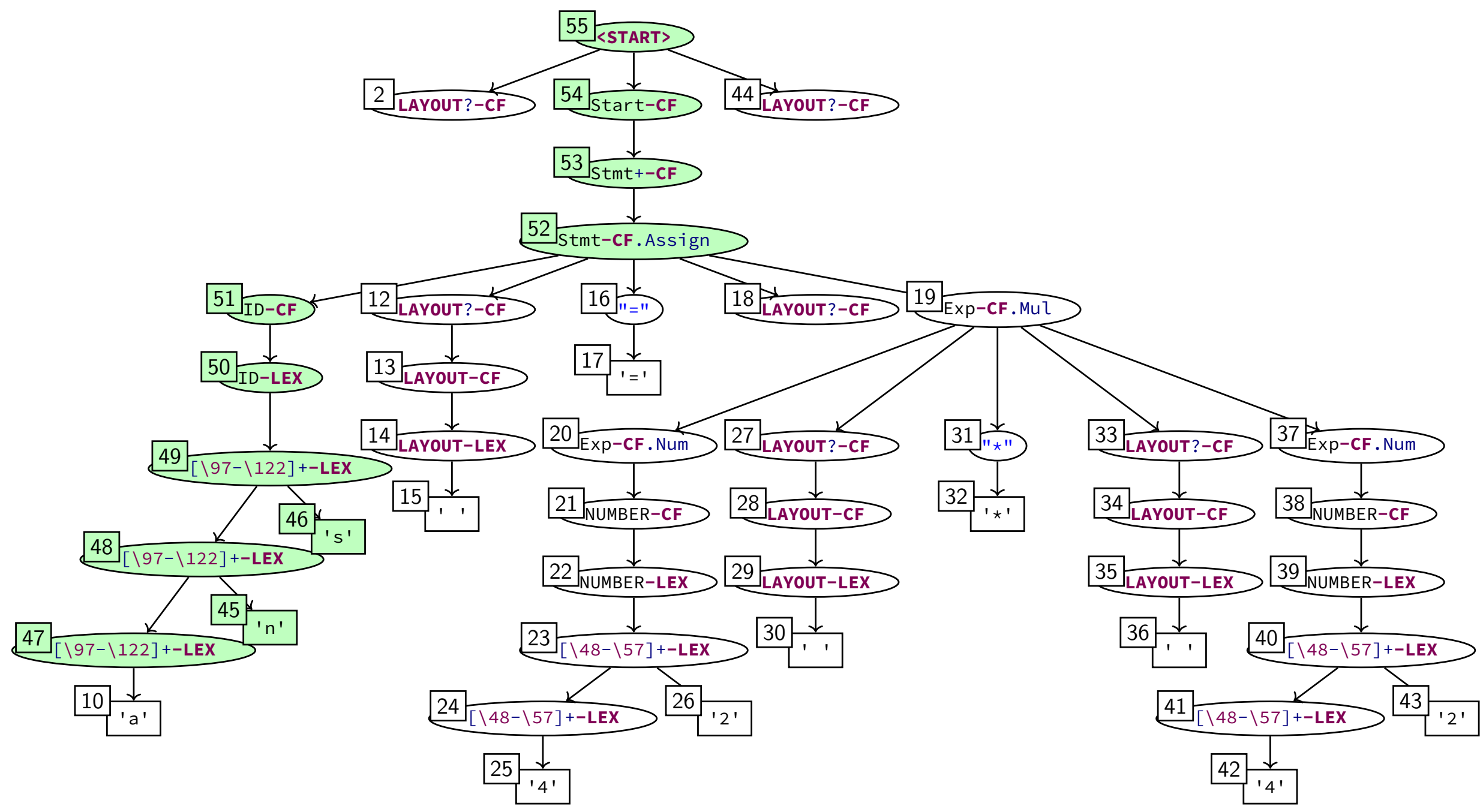

Figure 3.5: The resulting parse tree after first parsing " $a b=42 \star 42$ " and then incrementally parsing "ans $=42 \star 42$ ". The white nodes were created during the initial parse (see Figure 3.2) and the green nodes were created during the incremental parse (see Figure 3.4). 


\subsection{Non-determinism in ISGLR Parsing}

As explained for IGLR parsing in Section 2.3, non-deterministic parsing results in irreusable parse nodes, which can never be reused in a subsequent incremental parse if they end up on top of the input stack. While Wagner and Graham (1997b, §5) showed that non-deterministic parsing rarely occurs for token-based parsers, the parsing of character-level grammars relies more on non-deterministic parsing for disambiguation (Visser 1997, §2.2). In this section, we show some examples of this, using the grammar of Figure 3.1.

\subsubsection{Example With Layout Between Operators}

Consider a batch parse of the input " $a=x+y+z$ ". After parsing " $a=x+y$ ", the parse stack contains one parse node for each character, as in stack (1) in Figure 3.6.

With ' ' as the next character, the parser can take four successive Reduce actions. First, the top five nodes can be reduced to Exp-CF. Add (2), which can then be reduced together with four other parse nodes to Stmt-CF. Assign (3), which can, in turn, be reduced to Stmt-CF+ (4) and further to start (5). Figure 3.6 shows how these parse stacks look like, together with the parse nodes that are stored in the stack links. Of the five parse stacks, all of them can shift the ' ' except for stack (3):

(1) expects a '*' following the space, since Exp-CF.Mul has priority over Exp-CF.Add.

(2) expects a ' + ' following the space, since Exp-CF. Add is left-associative.

(4) expects another stmt following the space.

(5) expects the End-of-File (EOF) following the space.

The reason why so many parse stacks stay active is that the parser can only use one character as lookahead. It cannot look after the space to see that it is followed by a ' + '. After all, there could be an arbitrary number of spaces between the ' $y$ ' and the ' ' ', and with a different grammar, there could even be comments (which, in SDF3 grammars, are generally defined in a context-free way as part of the LAYOUT). Only after reducing the space via LAYOUT-LEX and LAYOUT-CF to LAYOUT?-CF and seeing that it is followed by a ' + ', the parser can discard all parse stacks except stack (2).

In the resulting parse tree, the Exp-CF. Add node corresponding to " $x+y$ " is an irreusable parse node because it was created when there were two parse stacks active, both expecting to shift layout (stacks (3)-(5) did not exist yet). The Exp-CF. Add node corresponding to " $x+$ $y+z$ " is also marked as irreusable. In this case, there was only one active parse stack, but the parser still had multiple actions: at the same time of creating this parse node, an empty LAYOUT?-CF node was created as well. This is because the latter parsing branch expects to find another ' + ' following the LAYOUT?-CF. ${ }^{2}$

Now consider an incremental parse with input " $a=x+y \star z$ ", where the second ' + ' changes to ' $*$ '. The Exp-CF. Add parse node corresponding to " $x+y$ " will be exposed on the top of the input stack and the parser will break it down since it is irreusable. However, most of the children of this parse node can be directly reused (except for the parse node corresponding to the space between ' $x$ ' and ' + ' because it was also irreusable, but that will be rebuilt), after which the parse stack looks exactly the same as stack (1). Before shifting the ' ' preceding the ' $*$ ', the parser can take the same four Reduce actions again. However, this

\footnotetext{
${ }^{2}$ This was also the case for the example in Section 3.1, but we ignored this there for simplicity. Also, note that SDF3 currently uses an LR(0) parse table generator. An LR(1) parse table would be able to see the EOF that follows it, and in that case, there is only one possible Reduce action and the empty LAYout?-CF parse node is not created. However, the parser can always be "tricked" into non-determinism by adding extra layout before the EOF: in that case, it will still try to create a layout node, this time being non-empty.
} 


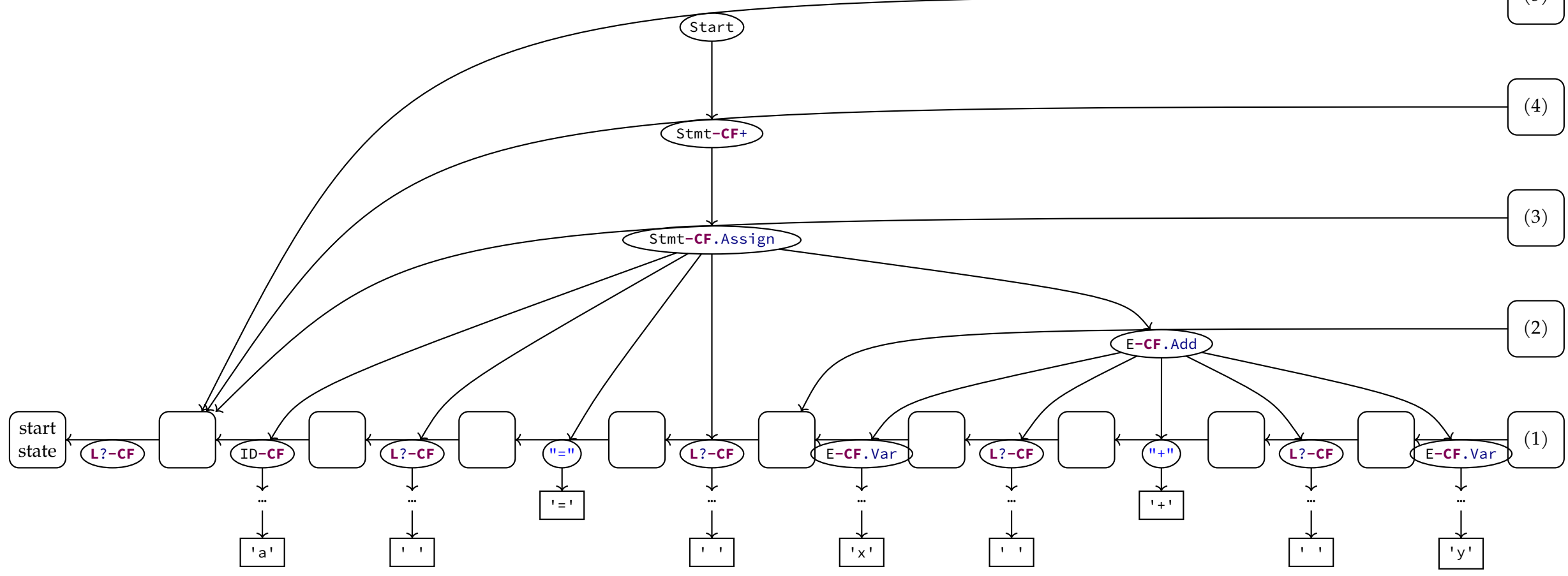

Figure 3.6: The active parse stacks after parsing " $a=x+y$ ", according to the grammar of Figure 3.1. The stacks are displayed right before a ' ' is shifted onto stacks (1), (2), (4), and (5). In the parse nodes, Exp is abbreviated to E and LAYout is abbreviated to L. 
time, only stack (1) will survive, and after processing ' ' and ' $z$ ' it creates an Exp-CF.Mul node. Note that this Exp-CF. Mul node is not irreusable because there is only one Reduce action possible: the grammar does not allow any (optional) layout following an Exp-CF. Mul node since it has the highest priority of all Exp production rules.

\subsubsection{Example With Layout Between List Items}

Consider a batch parse with input " $x=3 y=4 z=5$ ". After parsing " $x=3$ ", the parser is in a similar situation as in the start of the example in Section 3.2.1, shown as stack (1) in Figure 3.7(a). With one parse node on the parse stack for every character parsed so far, the parser is about to shift a ' '. Just like in the previous example, the parser can first reduce the current parse stack (1) multiple times, to Stmt-CF. Assign (2), Stmt-CF+ (3), and finally to start (4). At this point, all of these parse nodes are marked as irreusable, because stacks $(1),(3)$, and (4) can also shift a space as second action:

(1) expects a ' $\star$ ' or ' + ' following the space.

(3) expects another stmt following the space (this parse stack survives).

(4) expects the EOF following the space.

After parsing " $x=3 y=4$ ", the parse stack will look like stack (5) in Figure 3.7(b). Here, a similar thing happens as with the first statement: the top five parse node are reduced to Stmt-CF.Assign (6), after which the top three parse node are reduced to Stmt-CF+ (7), which is in turn reduced to start (8). Again, because a space follows next, stacks (5), (7), and (8) expect to shift it, so the parse nodes created from these reductions are again all irreusable.

The same thing happens when the parser creates the Stmt-CF+ parse node that spans the entire input, after also parsing the final " $z=5$ ". In the resulting parse tree, all parse nodes corresponding to Stmt-CF+ and Stmt-CF. Assign production rules are marked as irreusable. Now consider an incremental parse of the input " $x=3 y=4 z=7$ ". Even though only the very last character has changed, all stmt nodes will be broken down because they are irreusable and exposed on top of the input stack.

Do note that adding semicolons $(' ; ')$ at the end of a stmt production rule makes sure that a stmt-CF. Assign parse node is not marked as irreusable. ${ }^{3}$ The semicolon cannot be parsed as anything else than the end of a statement, and since it always consists of exactly one character, the parser does not need to consume any further characters before it can create a parse node for the statement. Similarly, blocks of statements enclosed in curly braces ( ' $\{$ ' and ' $\}$ ') are also not irreusable.

\subsubsection{Example With Identifier Versus Keyword}

For this example, we will extend the grammar of Figure 3.1 with two new production rules as shown in Figure 3.8. We add a return statement (stmt. Return) that consists of the keyword "return" and an expression (Exp). Note that this keyword could also be parsed as an identifier (ID), according to the rule ID $=[a-z]+$. Therefore, we also add a $\{$ reject $\}$ rule to disambiguate this.

Consider a batch parse of the input "return ab". The "return" part can be parsed in two ways: either as the keyword "return" (1) or as [\97-\122]+-LEX (2). Figure 3.9(a) shows these two parse stacks right after shifting the final ' $n$ '.

Following this Shift action, multiple Reduce actions follow, of which the final result is shown in Figure 3.9(b). First, the six character nodes of parse stack (1) are reduced to the

\footnotetext{
${ }^{3}$ Of course, before a parser can actually reuse them, they must still pass the state matching test, but that will only fail when the context changed.
} 


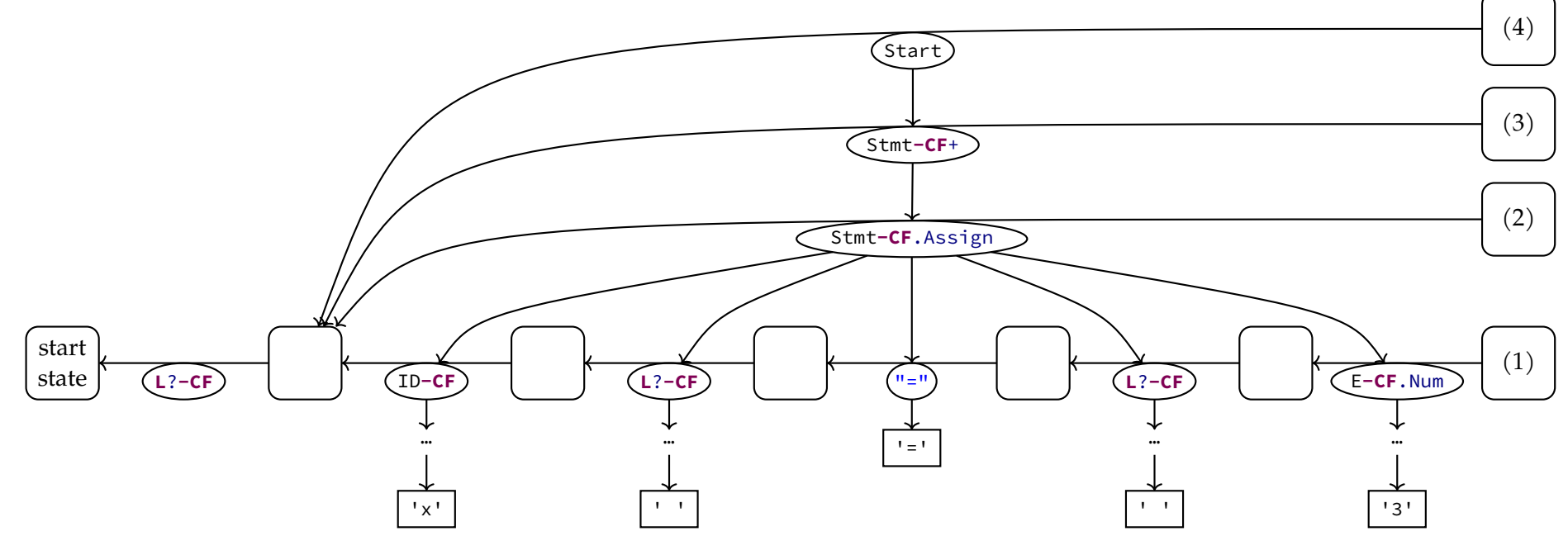

(a) The active parse stacks after parsing " $x=3$ ". The stacks are displayed right before a ' ' is shifted onto stacks (1), (3), and (4).

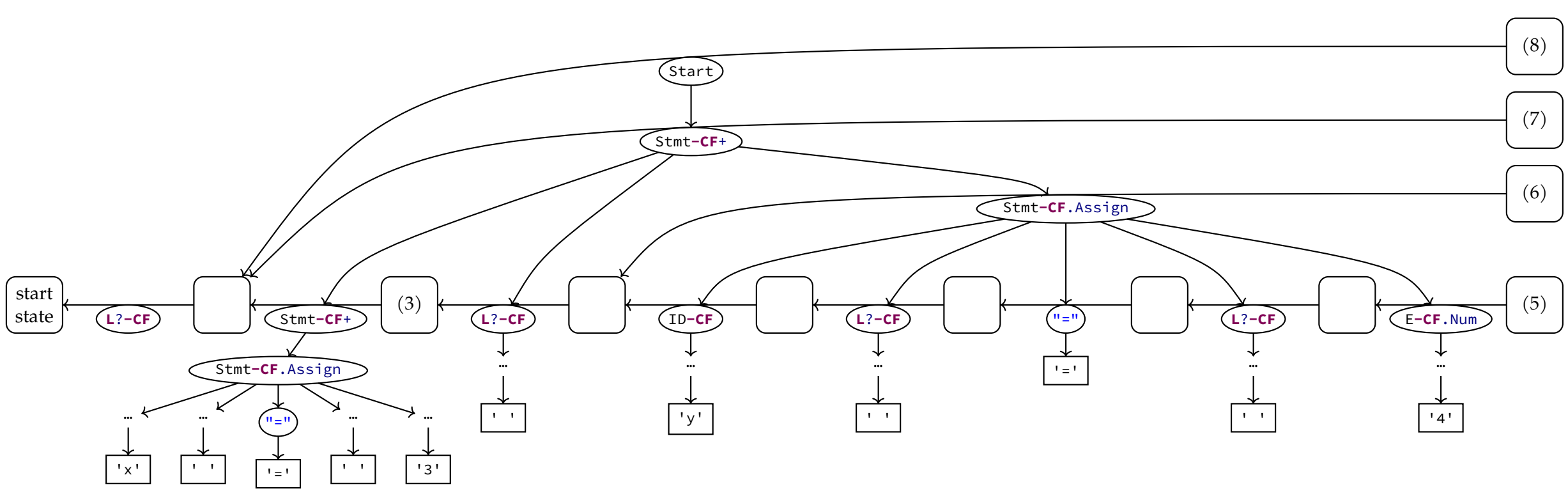

(b) The active parse stacks after parsing " $x=3 y=4$ ". The stacks are displayed right before a ' ' is shifted onto stacks (5), (7), and (8).

Figure 3.7: The active parse stacks at two points during the parsing of " $x=3 y=4 z=5$ ", according to the grammar of Figure 3.1. In the parse nodes, Exp is abbreviated to E and LAYOUT is abbreviated to $L$. 


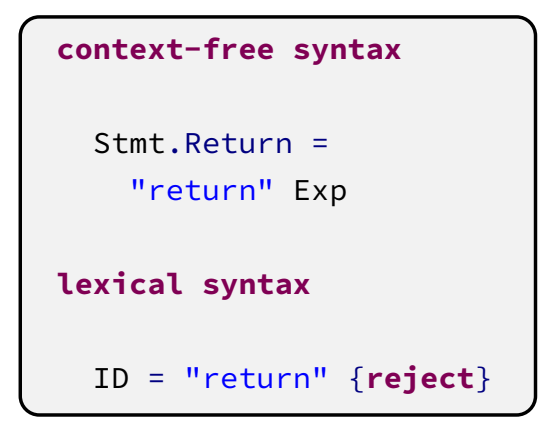

(a) The production rules, defined using high-level SDF3.

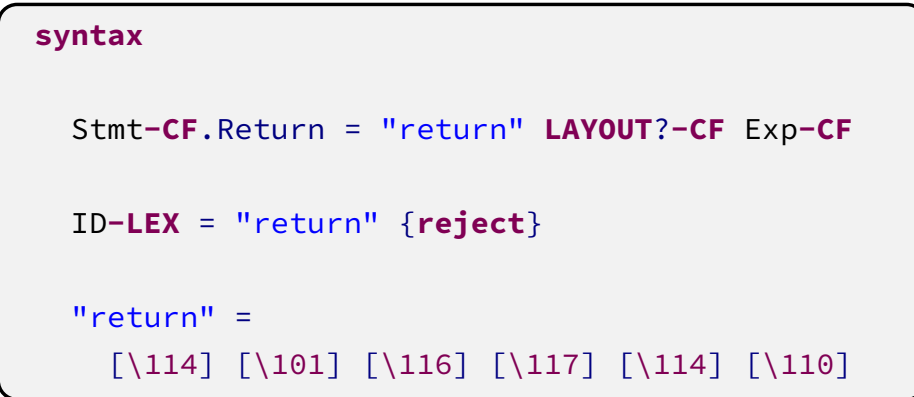

(b) The same production rules, where grammar normalization transformed them to kernel syntax.

Figure 3.8: Two production rules, added to the grammar of Figure 3.1, specified using SDF3 syntax. The first rule introduces a return statement and the second rule prevents the keyword "return" to be parsed as an identifier.

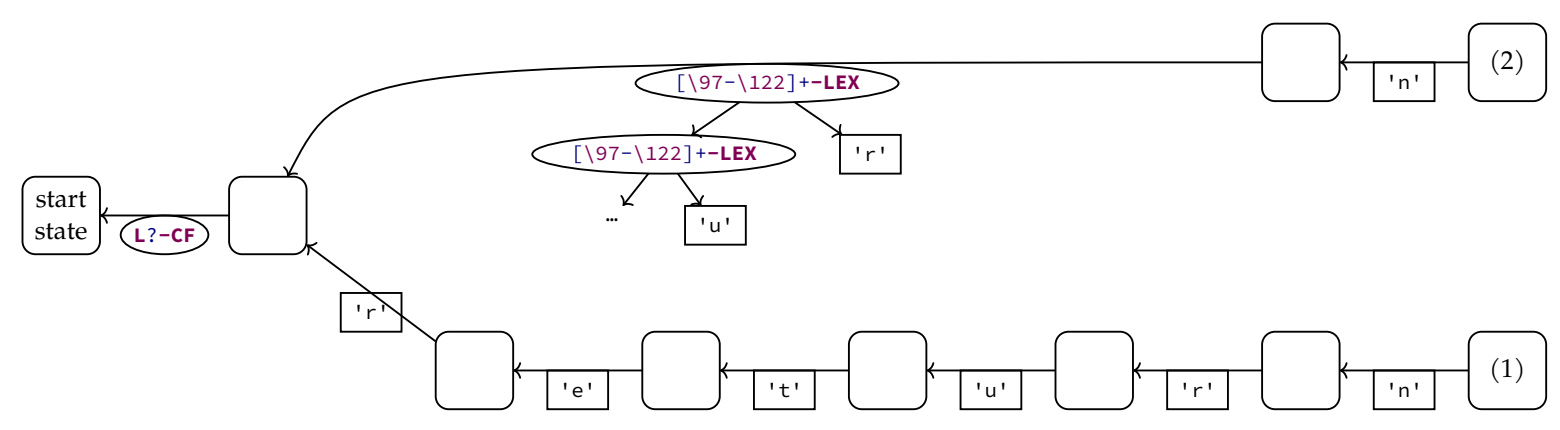

(a) The active parse stacks after parsing "return". The stacks are displayed right after shifting the ' $n$ '.

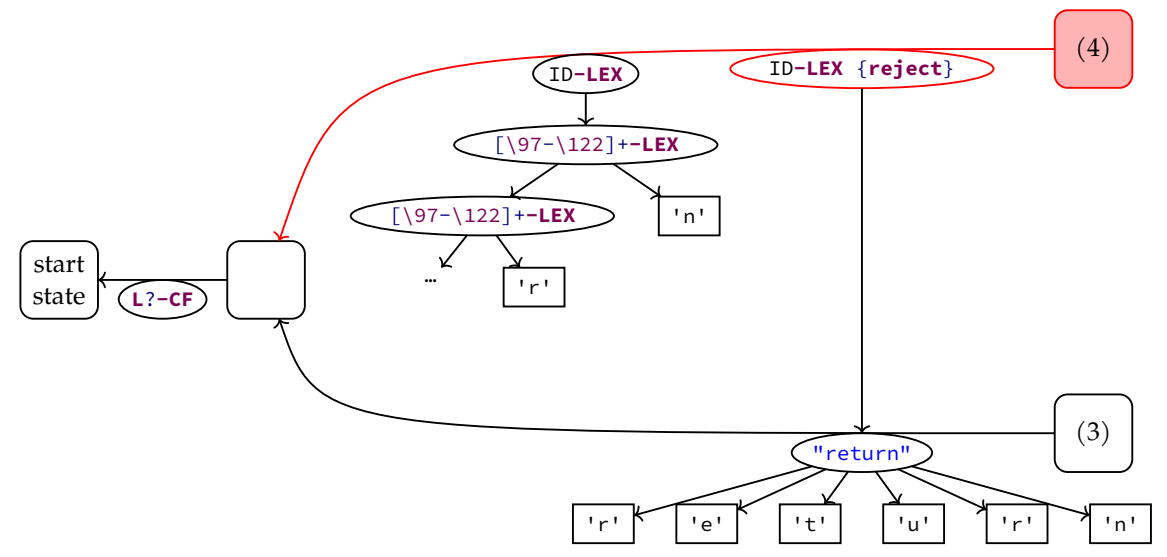

(b) The active parse stacks after parsing "return". The stacks are displayed after processing all possible Reduce actions based on Figure 3.9(a). The stack link of stack (4) contains two possible derivations for the same sort. This link is drawn in red because the second derivation marked it as rejected. Since stack (4) has no remaining valid stack links, the parser will no longer consider it for applying new actions. Therefore, the following ' ' can only be shifted onto stack (3).

Figure 3.9: The active parse stacks at two points during the parsing of "return ab", according to the grammar of Figure 3.1, extended with the production rules of Figure 3.8. In the parse nodes, LAYOUT is abbreviated to L. 
keyword "return" (3). Then, the [\97-\122]+-LEX parse node of stack (2) is reduced to ID-LEX (4). The "return" is also reduced to ID-LEX, because of the \{reject $\}$ rule. Note how this last reduction merges into stack (4) since it is another way of parsing the sort ID-LEX. This marks the stack link of stack (4) as rejected, which means that the parser will no longer consider it as an active parse stack since it has no remaining valid stack links.

Following these Reduce actions, parse stack (3) continues by shifting a ' ' as part of the LAYOUT?-CF of the production rule for Stmt-CF. Return. In the resulting parse tree, the parse node corresponding to the "return" keyword is marked as irreusable, because it was created while the parser was also exploring the possibility that it could have been an identifier.

Now consider an incremental parse for the input "return ans". The parser will definitely break down the Stmt-CF. Return parse node because its last child contains changes, making the "return" parse node end up on top of the parse stack. Because this is an irreusable parse node, the "return" keyword will need to be parsed from scratch.

\subsection{Valid Parse Node Reuse}

The ISGLR parser can only reuse an unchanged parse node if the context surrounding this node allows this. Following the approach of the IGLR parser by Wagner and Graham (1997b) as described in Section 2.3, we use a state matching test to check the context to the left and we test if the lookahead has changed to check the context to the right. For the lookahead test, we only need to test one character ahead in a deterministic setting. In order to also check the lookahead following a non-deterministic region, we will break down irreusable parse nodes that end up on top of the input stack to reconsider all possible ways of parsing that part of the input, as we discussed in Section 3.2.

In SDF3, it is possible to construct grammars that do not have optional layout inserted between context-free symbols, which avoids most occurrences of non-deterministic parsing. Figure 3.10 shows such a grammar, defined using kernel syntax, so that no optional layout is inserted during grammar normalization. In this expression grammar, the identifiers and operators always consist of exactly one character. This makes a scannerless parser behave exactly in the same way as a token-based parser would. We use this grammar to show two examples of how the state matching test (Section 3.3.1) and the lookahead test (Section 3.3.2) prevent invalid reuse of parse nodes in the ISGLR parsing algorithm.

\subsubsection{State Matching Test}

Consider a batch parse with input " $x+y \star z$ ". The priorities in the grammar of Figure 3.10 make sure that the resulting parse tree has the Exp-CF. Mul node as the right child of the Exp-CF. Add node, i.e., it could be parenthesized as " $x+(y \star z)$ ". Note that this input is parsed fully deterministically thanks to these priorities, so none of the parse nodes is marked as irreusable. This is regardless of the conflicts in the parse table, as we will explain in Section 3.3.3.

Now consider an incremental parse of the input " $x \star y \star z$ ". The " + " changes into a " $\star$ ", so the Exp-CF. Add parse node from the previous parse is broken down. After parsing " $x \star$ ", the parse stack contains one Exp-CF.Var node and one "*" node, as shown in Figure 3.11. Parse state 6 is on top of the stack, which is different from the first parse after the parsing " $x+$ ": the node for " $y \star z$ " (Exp-CF.Mul) was created on top of state 8 during the previous parse. Because this parse node fails the state matching test, it cannot be directly reused and it is broken down. The node for " $y$ " (Exp-CF.Var) and the node for " $\star$ " (" $* ")$ are also broken down because of a failing state matching test. After that, incremental parsing continues as normal, with a resulting parse tree that can be parenthesized as " $(x \star y) \star z$ ". 


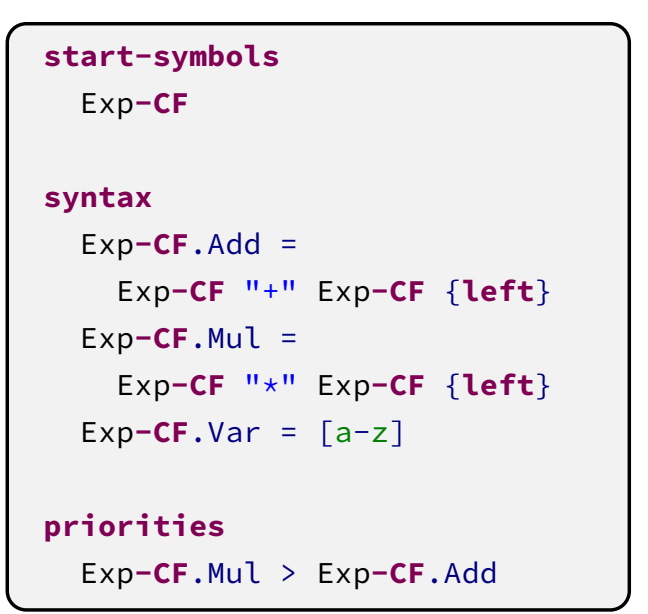

(a) The grammar, defined using SDF3 kernel syntax.

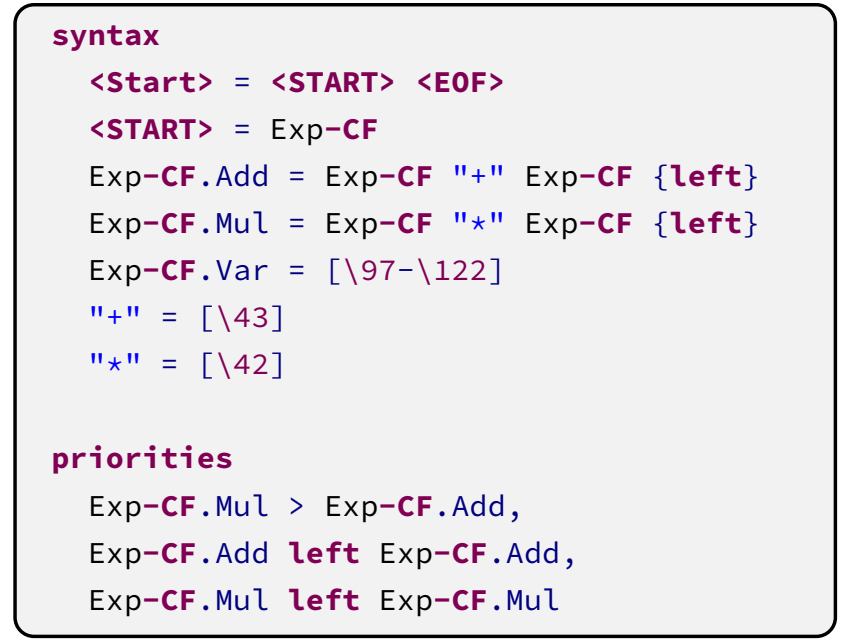

(b) The same grammar, after grammar normalization.

\begin{tabular}{|c|c|c|c|c|c|c|c|c|c|c|c|}
\hline \multirow{2}{*}{ state } & \multicolumn{5}{|c|}{ actions } & \multicolumn{6}{|c|}{ gotos } \\
\hline & {$[+]$} & {$[*]$} & {$[a-z]$} & $\langle$ <EF $\rangle$ & all & "+" & "*" & E.A & E.M & E.V & 〈START $\rangle$ \\
\hline 0 & & & $S(1)$ & & & & & 3 & 2 & 2 & 4 \\
\hline 1 & & & & & $R(\mathrm{E} . \mathrm{V})$ & & & & & & \\
\hline 2 & $S(7)$ & $S(5)$ & & & $R(\langle$ START $\rangle)$ & 8 & 6 & & & & \\
\hline 3 & $S(7)$ & & & & $R(\langle$ START $\rangle)$ & 8 & & & & & \\
\hline 4 & & & & Accept & & & & & & & \\
\hline 5 & & & & & $R(" \star ")$ & & & & & & \\
\hline 6 & & & $S(1)$ & & & & & & & 9 & \\
\hline 7 & & & & & $R("+")$ & & & & & & \\
\hline 8 & & & $S(1)$ & & & & & & 10 & 10 & \\
\hline 9 & & & & & $R(\mathrm{E} . \mathrm{M})$ & & & & & & \\
\hline 10 & & $S(5)$ & & & $R(\mathrm{E} . \mathrm{A})$ & & 6 & & & & \\
\hline
\end{tabular}

(c) The parse table of the grammar, as generated by SDF3. Shift actions are abbreviated to ' $S$ ' and Reduce actions to ' $R$ '. The sort and constructor names have been abbreviated to single letters. Note that the parse table generator of SDF3 produces one goto state per production rule (Visser 1997, §5.4), rather than one per sort (as for LR generators). The Reduce actions are valid for any lookahead since SDF3 currently uses LR(0), and we will prefer a Shift action over a Reduce action to resolve the conflicts, as will be explained in Section 3.3.3.

Figure 3.10: A small expression grammar defined using SDF3 kernel syntax, so that it does not get optional layout (LAYOUT?-CF) in between production symbols or surrounding the start symbol during grammar normalization.
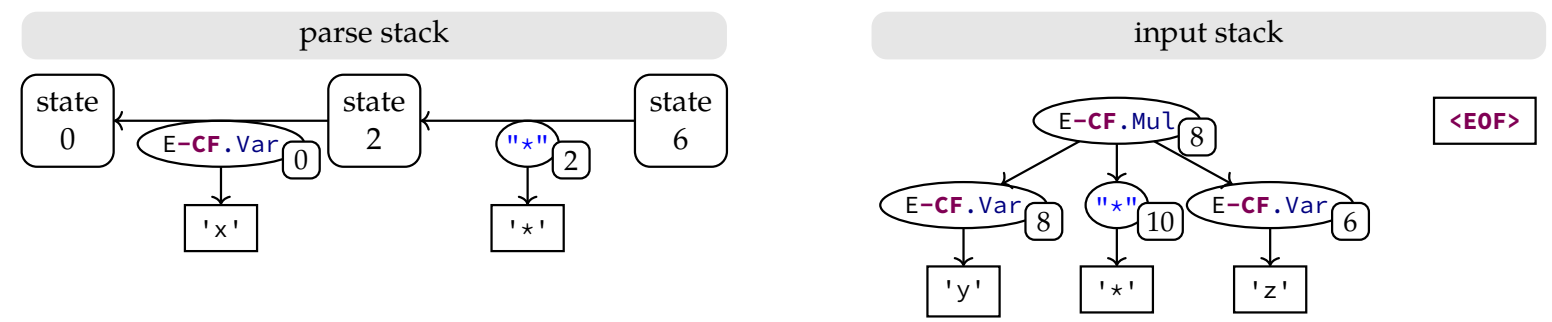

Figure 3.11: The active parse stack and remaining input stack during an incremental parse from " $x+y \star z$ " to " $x \star y \star z$ ", after parsing " $x \star$ ", according to the grammar of Figure 3.10. The parse state references stored in parse nodes are shown as labels at the bottom right of a node. In the parse nodes, Exp is abbreviated to $\mathrm{E}$. 


\subsubsection{Lookahead Test}

Consider a batch parse of the input " $x+y+z$ ". Because the Exp-CF. Add production rule is leftassociative, the resulting parse tree can be parenthesized as " $(x+y)+z$ ". Just as in the previous example, this input is parsed fully deterministically, so none of the parse nodes is marked as irreusable.

Now consider an incremental parse with input " $x+y \star z$ ". The priorities in the grammar of Figure 3.10 dictate that the new input should be parsed as " $x+(y \star z)$ ". After updating the second " + " to " $\star$ ", a parser without the lookahead test would see that the parse node corresponding to " $x+y$ " is not changed, and blindly reuse it. However, this parse node should actually be broken down because the lookahead changed, so the parser should take a different action this time. Instead of reducing to Exp-CF. Add, it should now shift the ' $\star$ '.

In the ISGLR parsing algorithm, we implement the lookahead test by considering the character preceding an edit as "changed" when calculating whether the parse node at the top of the input stack contains a change. In this example, this means that the parser considers the " $y$ " preceding the new " $\star$ " as changed. This will trigger the breakdown of the parse node corresponding to " $x+y$ " and allow the parser to pick a different action. For this approach, we do not need to remember what the previous lookahead was for every parse node, at the cost of potentially breaking down slightly more parse nodes than necessary.

\subsubsection{Conflicts in This Parse Table}

In this section, we have ignored the Shift/Reduce conflicts in the parse table of Figure 3.10(c). These conflicts are present because SDF3 currently uses an $\operatorname{LR}(0)$ parse table generator. In an LR(1) parse table, the Reduce actions would not overlap with the Shift actions.

For our examples, this means that the parser sometimes tries to reduce to Exp-CF.Add or <START> while it should only do a Shift action, so it temporarily creates a second parse stack, similar to the examples in Sections 3.2.1 and 3.2.2. However, because the Shift action can only be executed on the first parse stack, the other parse stack is immediately discarded. None of the irreusable parse nodes created during the small period where multiple parse stacks were active, ends up in the final result, because their parse stacks are discarded.

\subsection{ISGLR Parsing Algorithm}

In this section, we present the full ISGLR parsing algorithm, broken up into separate functions and procedures. The changes with respect to the SGLR parsing algorithm by Visser (1997) (and in particular, the implementation by Denkers $(2018, \S 2.5)$ ) are indicated using a vertical bar in the left margin of the page and will be explained per procedure. These changes mostly follow the ideas of Wagner and Graham (1997b), with some exceptions: we have a different way of applying changes to the parse tree (Algorithms 1 and 3) and we work around the fact that the parse table generated by SDF3 does not support directly indexing the action table using a production rule instead of a character (Algorithms 4 and 5).

The ISGLR parsing algorithm uses the functions PeEK, PoP, and BREAKDown, that act on an incremental input stack. PeEK returns the node on top of the stack without removing it, Pop removes the node on top of the stack and returns it, and BREAKDown breaks down the top node of the input stack and pushes back its children in reverse order.

The PARSE function in Algorithm 1 has a different signature than Visser's algorithm: it now allows passing the input string and parse result of the previous version. These two new parameters are allowed to be empty, in which case we start a batch parse by initializing the input stack with only character nodes. In the case of an incremental parse, we calculate the DifF between the previous and current version of the input string according to Figure 3.3. 
We initialize the incremental input stack with the EOF marker $(\$)$ and the root node of the previous parse tree. We break down the previous parse tree along the spine between the root node and the changes at the start of the PARSESTEP procedure of Algorithm 2. Additionally, we define a global boolean variable to remember whether the parser is in multiple states, i.e., whether it is parsing non-deterministically. Finally, the parse loop has been adapted to use the incremental input stack instead of iterating over all characters in the input.

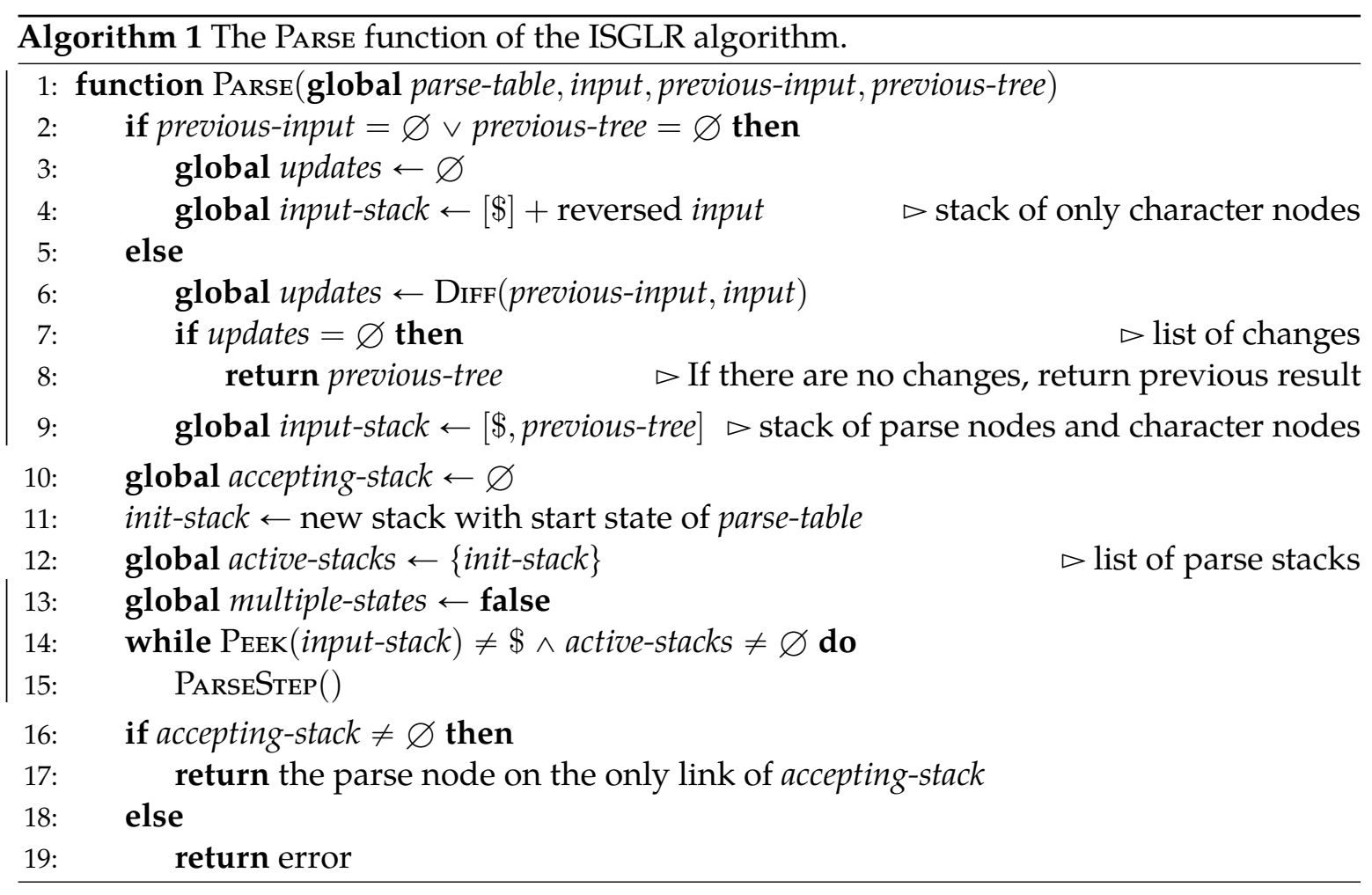

There are three changes to the PARSESTEP procedure in Algorithm 2. Firstly, the assignment to the variable current-character is removed, because we use the incremental input stack to get the lookahead instead. Secondly, the variable multiple-states is set to true if there is more than one active parse stack. Finally, we call the СнескUрDates procedure of Algorithm 3 before we start processing the for-actor stacks.

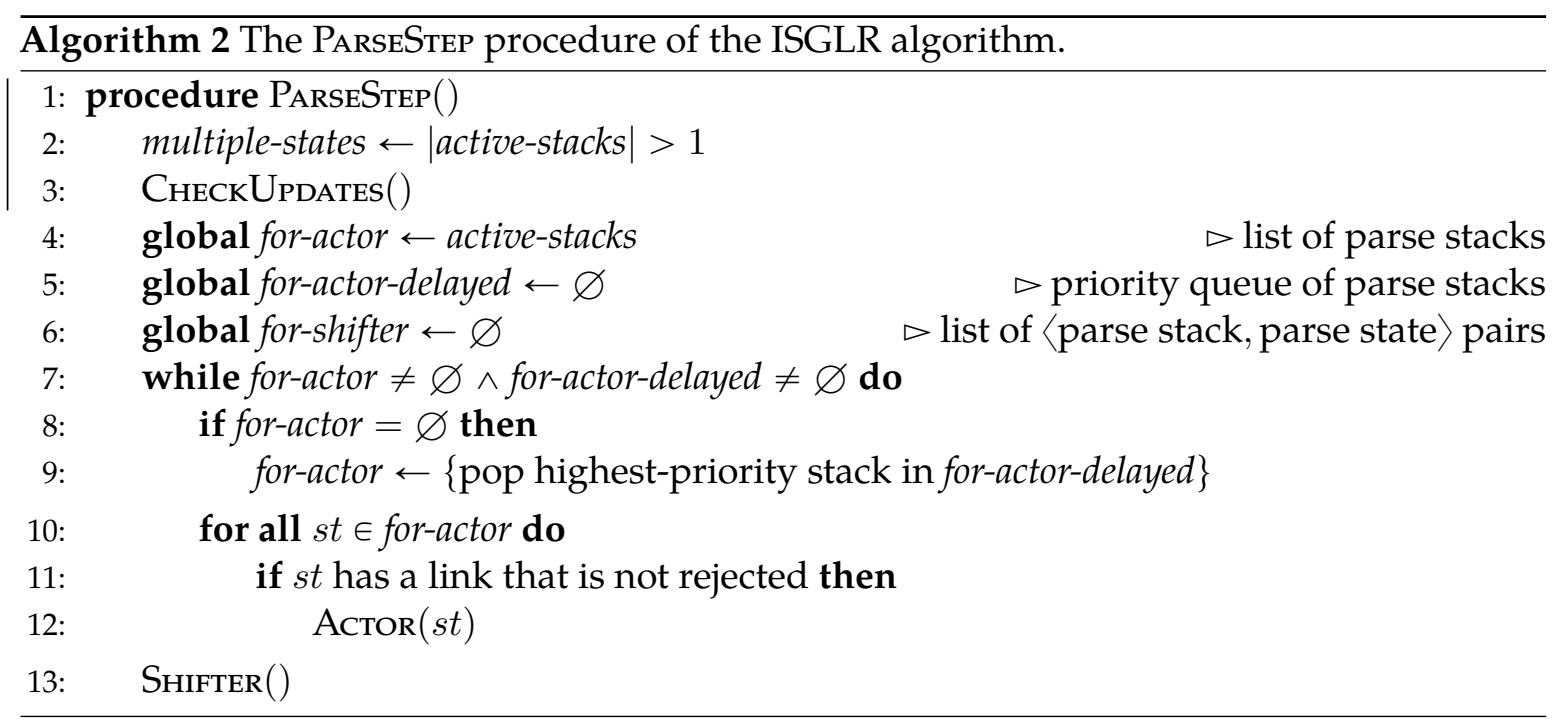


The СнескUрDATES procedure in Algorithm 3 checks whether the current lookahead contains any of the changes that were calculated by the diff. Firstly, it checks whether the current position of the parser is the start of a deletion. If this is the case, we pop all parse nodes that fall within the range of the deletion from the input stack. If the node at the top of the input stack covers the deletion only partially, we break it down and continue the loop for its children. After the deletion phase, we push all characters that are inserted by the change to the input stack. Secondly, in the case that the parse node on top of the input stack overlaps with any of the changes calculated by the diff, we break it down until this is no longer the case.

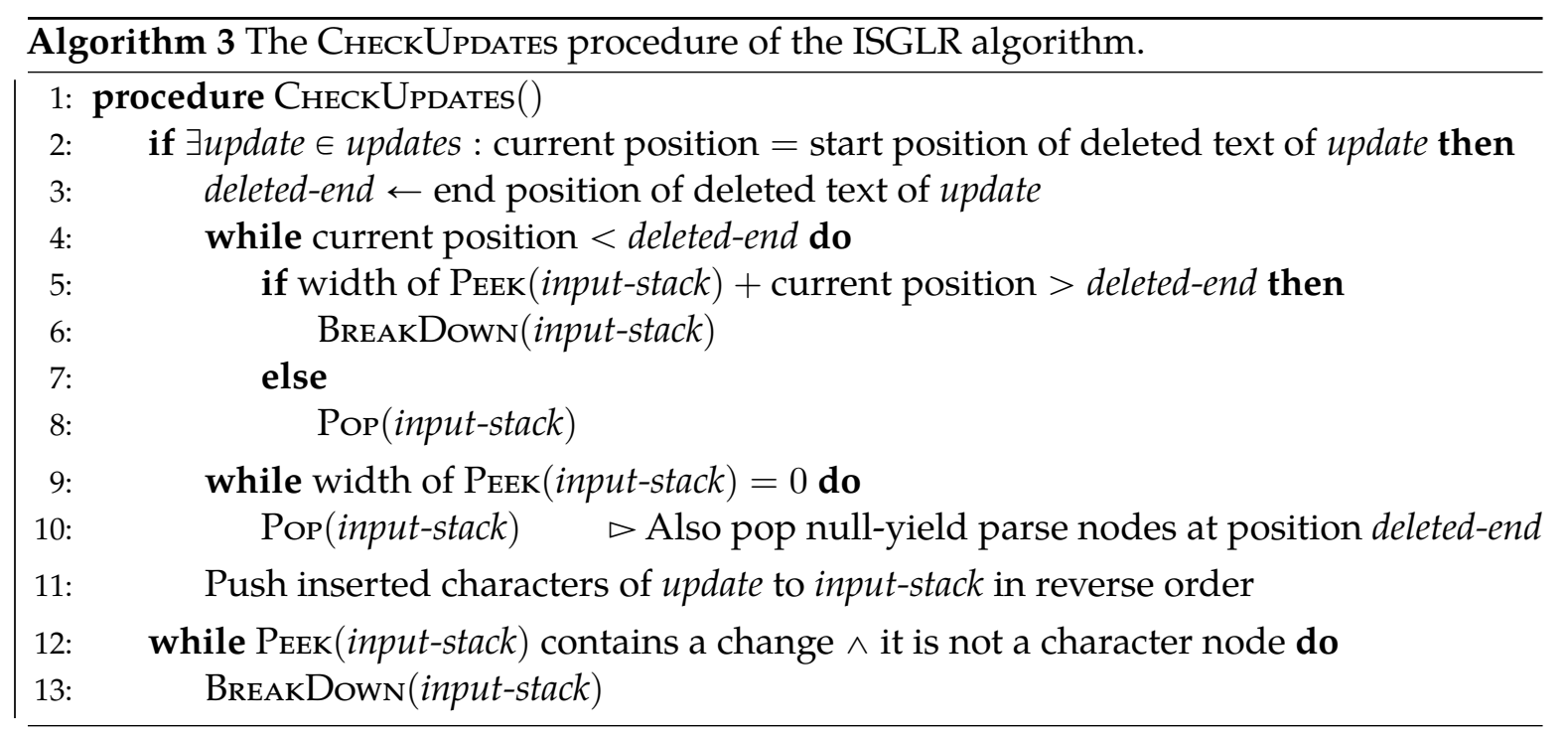

The Aстов procedure in Algorithm 4 has a different way of getting the actions from the parse table. Because the symbol of the top node in the incremental input stack can be either a terminal or not, the parse table can be indexed in different ways. This has been moved to a separate function BREAKDownUnTILVALIDActions which is shown in Algorithm 5. If that function needs to break down the current lookahead, any elements that we already added to for-shifter need to be updated. It is still valid to shift the new lookahead onto the parse stacks in for-shifter by construction of the parse table, but the stacks will go to a different state. Finally, if there are multiple possible actions, the multiple-states variable is set to true.

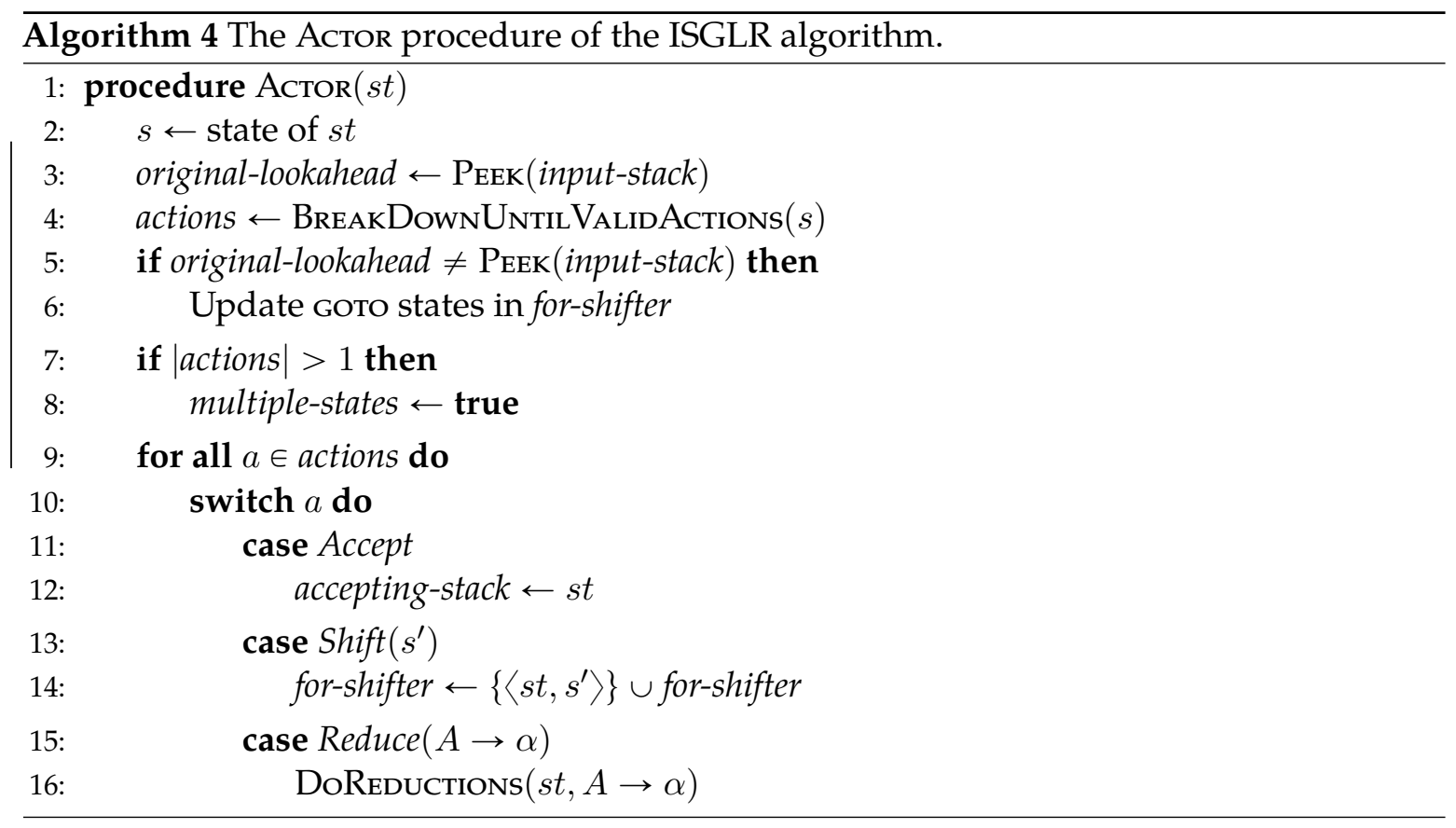


The BreakDownUntil ValidActions function in Algorithm 5 determines the possible actions for the parser based on the current top of the incremental input stack (called lookahead). We use the first character of the lookahead to index the action table of the parse table. If the lookahead is a character node, we don't enter the loop and immediately return the list of actions. If the lookahead is a parse node, we enter the loop to check if we can reuse it.

If the state matching test succeeds (see Section 3.3.1), we substitute the normal Shift action with a new one that uses the state from the goto table which matches the production rule of the lookahead. We also do an optimization here in the case that the only Reduce action would create a parse node without children and the leftmost descendant of the lookahead has the same production rule as this Reduce action. In this case, we can remove this action to avoid setting multiple-states to true, because this action will eventually result in the same parse node as the one that we want to shift.

We break down the lookahead if it is an irreusable parse node or when there are Shift actions. Note that at this point, we know that the state matching test failed, so if we can do different Shift actions based on the first character in the lookahead, this lookahead possibly needs to be parsed in a different way. If the lookahead is not irreusable and we only have Reduce actions, we can safely return the current list of actions, which saves us from breaking down the lookahead.

If the lookahead that we broke down had no children, this is similar to popping a node from the input stack, so we need to do two more things. Firstly, we check if the new lookahead contains any changes and break it down again until this is no longer the case because the next parse node on the input stack might span a part of the input that has changed. Secondly, we empty for-shifter because the parse node that these parse stacks want to shift no longer exists. These parse stacks will be recreated by Reduce actions with arity 0 . Note that this does not interfere with the optimization of line 10, because that optimization only occurs when the parser is not in multiple states, meaning that for-shifter must be empty by definition in that case.

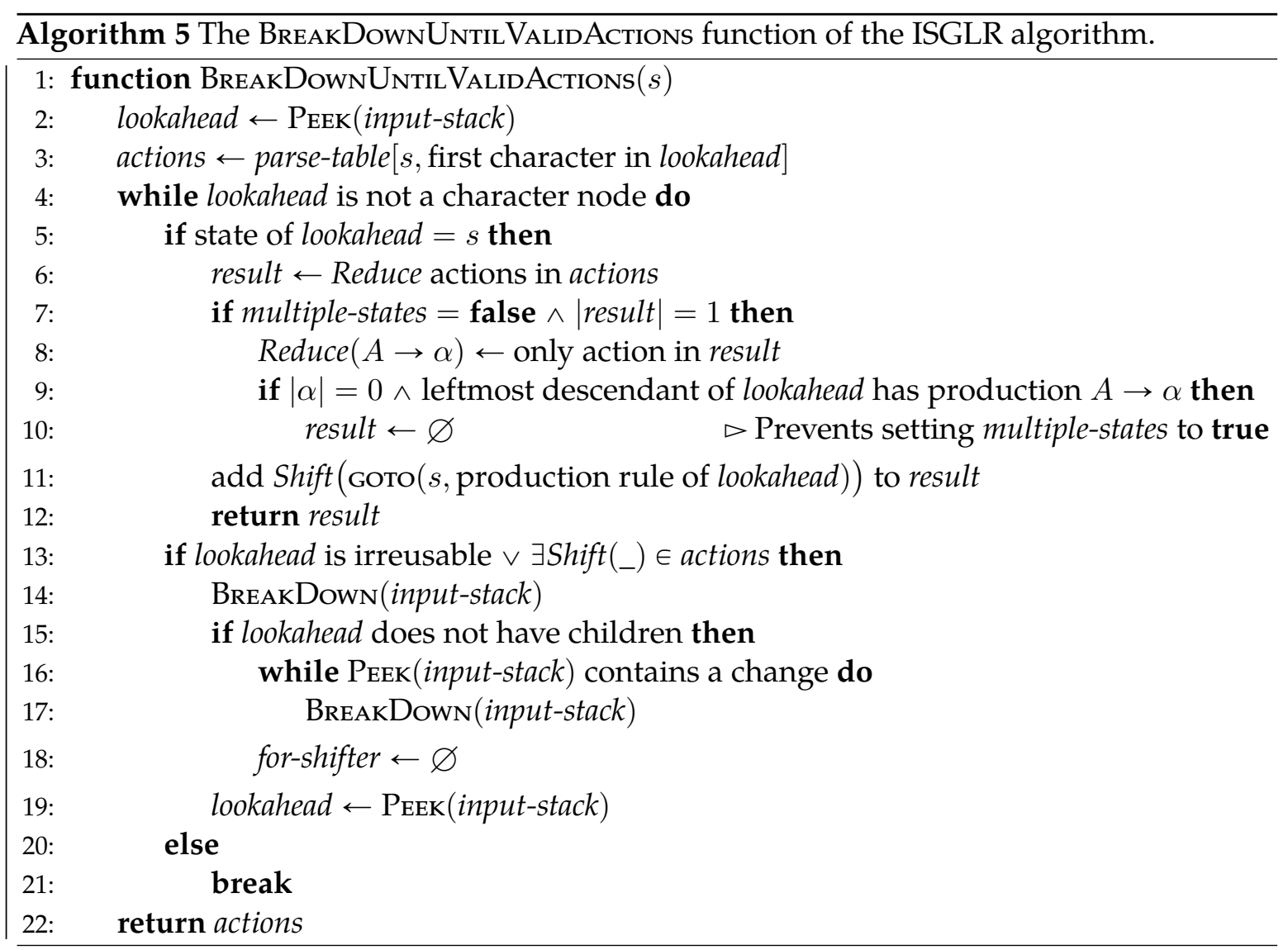


In DoReductions and DoLimitedReductions (Algorithms 6 and 8), the parser records that it is in multiple states when this reduction leads to more than one Goto state. This can occur when multiple parse stacks have previously been merged into a single stack, and the multiple valid paths would make the REDUCER procedure add multiple new stacks to for-actor.
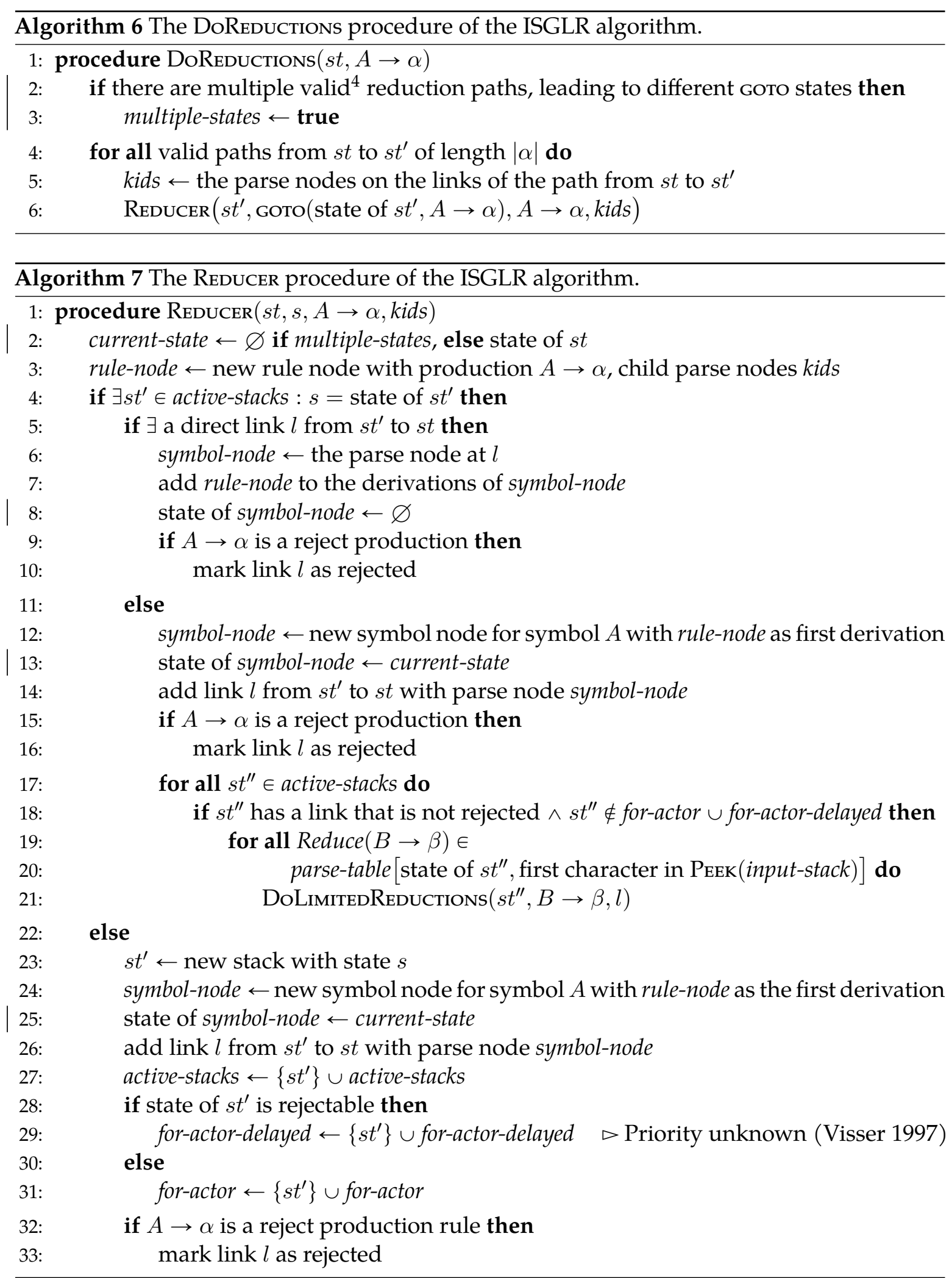

\footnotetext{
${ }^{4}$ These "valid" paths are those that do not include rejected links. Neither Visser (1997) nor Denkers (2018) mention this in the SGLR parsing algorithm, but Denkers added this in the JSGLR2 implementation to fix a reported issue: https://github.com/metaborg/jsglr/commit/5395a5870d51fd08578016fbdd0a756072799ed7
} 
The REDUCER procedure in Algorithm 7 has one change: we save the parse state of the current parse stack in the newly created rule-node (or no state if the parser is in multiple states, to mark it as irreusable).

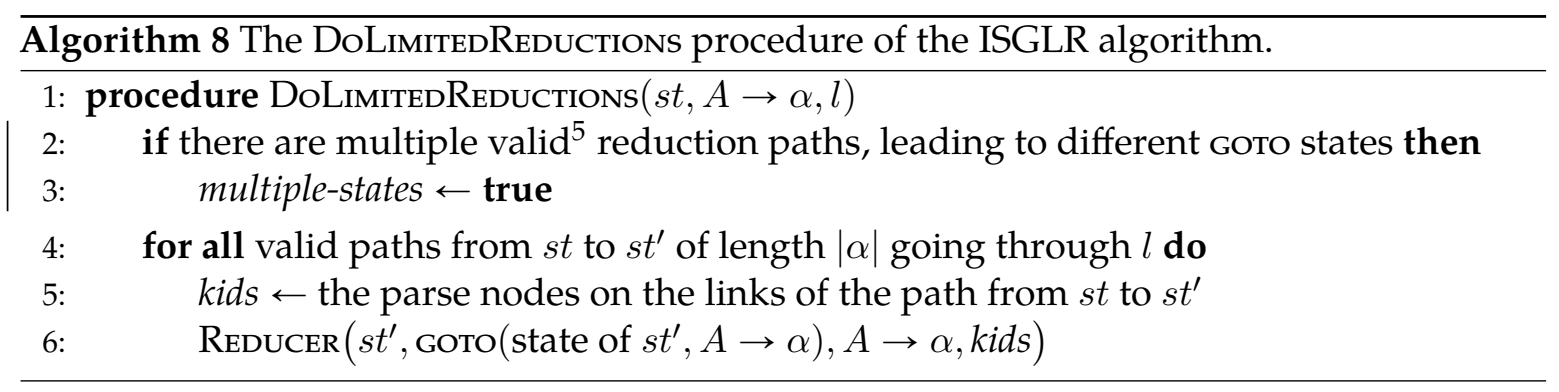

The SHIFTER procedure in Algorithm 9 has one main change. It no longer needs to create a new character node for the current character, but instead, we shift the character node or parse node that is at the top of the input stack.

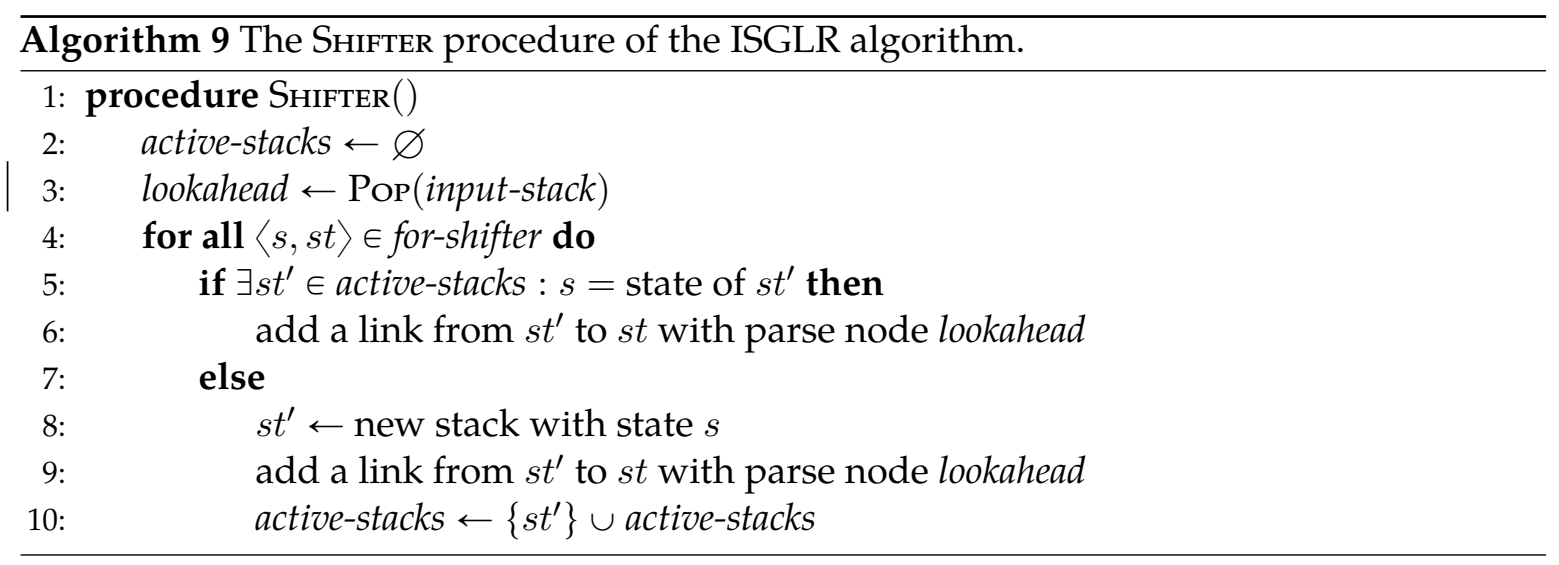

\subsection{Implementation in Modular Architecture}

In this section, we describe the implementation of the ISGLR parsing algorithm in the modular JSGLR2 implementation by Denkers (2018) (see Section 2.4.3). Figure 3.12 shows the changes to the parsing pipeline of JSGLR2. This figure also shows the Imploder and Tokenizer components, which will be discussed in detail in Chapter 4.

The implementation of the ISGLR parser in the modular architecture of JSGLR2 requires extensions for several data structures (input stack, parse state, and parse forest) and code components (parser, reduce manager, and parse forest manager). We discuss each of these in the paragraphs below, as well as how we manage the caching of previous results between incremental parses.

Input Stack The ISGLR parser requires a specialization of the input stream because it consumes a stream of parse nodes instead of a stream of characters. Also, the input stream should allow breaking down parse nodes. Because of this, the implementation of this component can be better described as an input stack, rather than a stream.

The implementation of the incremental input stack also handles the processing of changes as shown in the CHескU PDATES procedure in Algorithm 3. This encapsulation simplifies the other parsing procedures since they can always assume that the top of the input stack does not contain any changes.

\footnotetext{
${ }^{5}$ See footnote 4 in DoReductions.
} 


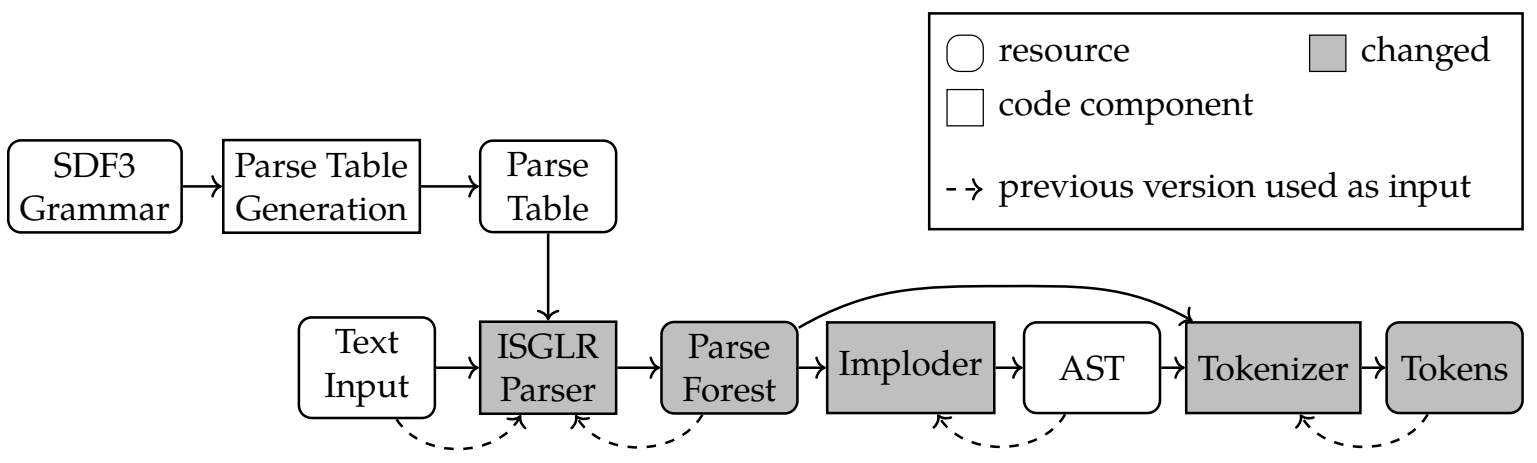

Figure 3.12: The parsing pipeline in Spoofax, updated from Figure 2.10 for incremental parsing. The top row of the pipeline is executed during language development, while the bottom row is executed every time a file is parsed. The dashed arrows indicate that a previous version of this resource is required as input to the incremental components.

Parse State The Parsestate class for the ISGLR parser requires two modifications. Firstly, the basic input stream is replaced with the incremental input stack. Secondly, it needs to store the multiple-states global variable, which determines whether newly created parse nodes are irreusable or not.

Parse Forest (Manager) The Parseforest class and its subclasses have two modifications for the ISGLR parser. Firstly, each ParseNode stores the parse state that the parser was in during the creation, or no parse state if the parser was in multiple states, making the parse node irreusable. Secondly, we store the width of each ParseForest, i.e., the number of characters in its yield, to optimize the processing of updates and the imploding and tokenization steps. Because of these extensions to the ParseForest, the ISGLR parser also requires a specialized ParseForestManager, which makes sure that all the required data is passed to the constructor of the ParseForest classes.

Parser and Reduce Manager The changes for the ISGLR parsing algorithm with respect to SGLR, as indicated in Section 3.4, have been implemented in an extension of the Parser class. At the start of the PARSE procedure, it instantiates the new data structures. In the AстоR procedure, getting actions from the parse table is extended to work if the current top of the input stack is a parse node instead of a character. Also, this procedure triggers the breakdown of the parse node that is currently on top of the input stack if it cannot reuse this parse node or if it finds no applicable actions in the parse table. Keeping track of the multiplestates variable requires changes in multiple places: at the start of the PARSESYMBOL procedure, after fetching the actions in the AстоR procedure, and after calculating the number of valid reduction paths in the Do(Limited)Productions procedures (in the Reducemanager class).

Cache We set up the ISGLR parsing algorithm and the Imploder and Tokenizer components such that they take the previous result as input, i.e., they are agnostic of how these results are cached. Users of the JSGLR2 implementation are free to determine their caching strategy, but we also implemented a default caching strategy that can be used. This strategy maintains four HashMaps, one for each resource that needs to be cached (see the dashed arrows in Figure 3.12). These HashMaps are indexed using a file name, combined with some metadata of the parsing request. They are only updated when the parser produces a valid result, i.e., this caching strategy ignores parse failures. 
This page is intentionally left blank. 


\section{Chapter 4}

\section{Incremental Post-Processing}

After parsing, the JSGLR2 implementation performs two post-processing tasks, as shown in Section 2.4.3 and Figure 2.10, to simplify the integration of the parser into an editor. These tasks can also be performed incrementally, making use of the result of the Incremental Scannerless Generalized LR (ISGLR) parser, as shown in Section 3.5 and Figure 3.12. This chapter describes these tasks and shows how we made them incremental.

First, the imploder (Section 4.1) reduces the parse forest from a Concrete Syntax Tree (CST) into an Abstract Syntax Tree (AST). The AST only contains elements that are useful for further processing, discarding any layout and redundant lexical elements. The AST is used for type checking and code transformations. Because the imploding of a parse node does not require information about its context, the incremental imploding algorithm can directly reuse previous imploding results for unchanged parse nodes.

Second, the tokenizer (Section 4.2) transforms the parse forest to a list of tokens and attaches these tokens to the AST. It uses the production rule that was used to create a parse node to determine the type of the token. The tokens are used for syntax highlighting and error reporting. Similar to imploding, the incremental tokenization algorithm can reuse previous tokenization results for unchanged parse nodes. However, instead of storing absolute positions in tokens, we create a tree-shaped data structure that allows calculating the absolute positions on the fly.

\subsection{Imploding}

The parse forest that the parser produces is a Concrete Syntax Tree (CST), containing parse nodes for all kinds of production rules of the context-free grammar. However, many of these parse nodes are not necessary for further processing steps of the parse result, such as transformation and code generation in Stratego (Bravenboer et al. 2008; MetaBorg 2016) or type checking in Statix (van Antwerpen et al. 2018; MetaBorg 2016). For example, layout and literals do not add any semantic meaning to the program. Therefore, these elements are discarded when the imploder transforms the CST into an AST.

As an example, see the imploded AST of the parse tree in Figure 3.2 in Figure 4.1. In the CST of Figure 4.1(a), all parse nodes that are not needed to build the AST are left out. The remaining relevant nodes can be divided into three categories: those representing lists (node 4 ), those containing constructors (nodes $5,39,20,37$ ), and characters that are not part of literals or layout (nodes $10,11,25,26,42,43$ ). As shown in Figure 4.1(b), the list nodes are converted into lists in the AST using square brackets $([\ldots])$, the parse nodes with constructors are converted into applications of their children, and the characters are imploded into strings. This last step is specific to scannerless parsing since parsing techniques that require a lexer would already have combined separate characters into larger tokens. 

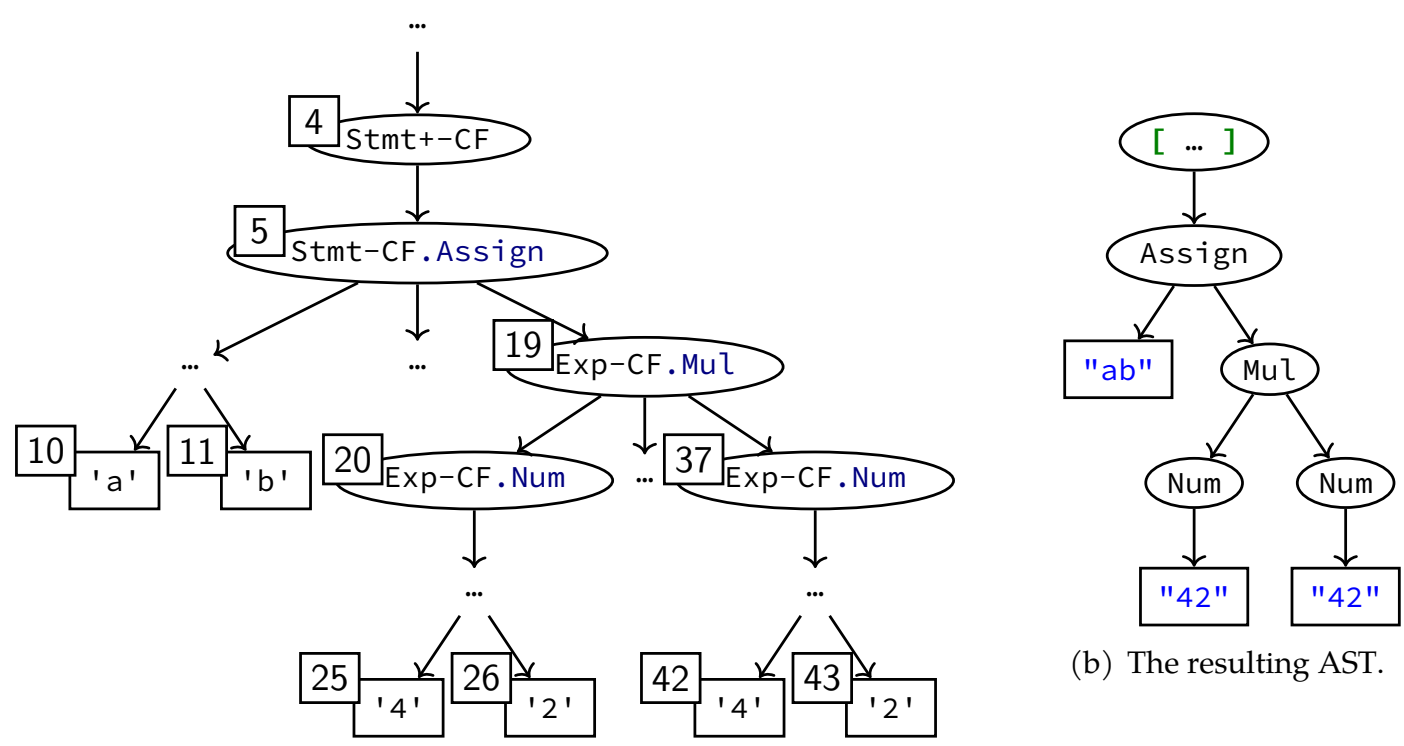

(b) The resulting AST.

(a) The relevant parse nodes of the CST to build the AST.

Figure 4.1: The imploded AST corresponding to the parse tree of Figure 3.2. In textual form, this AST would be written as [Assign("ab", Mul (Num("42"), Num("42")))] .

\subsubsection{Imploding Algorithm}

The current, non-incremental imploder in JSGLR2 works as shown in Algorithms 10 to 12.

The IMPLODE function of Algorithm 10 will decide how to implode the given parse-tree based on its grammar production. If the production is context-free, the imploder will recursively implode the children of parse-tree. In the case that parse-tree is an ambiguous parse node, the imploder will do this for all alternatives. If the production is not context-free, the imploder creates a leaf of the AST. This leaf is empty in the case that the production is a literal or layout production.

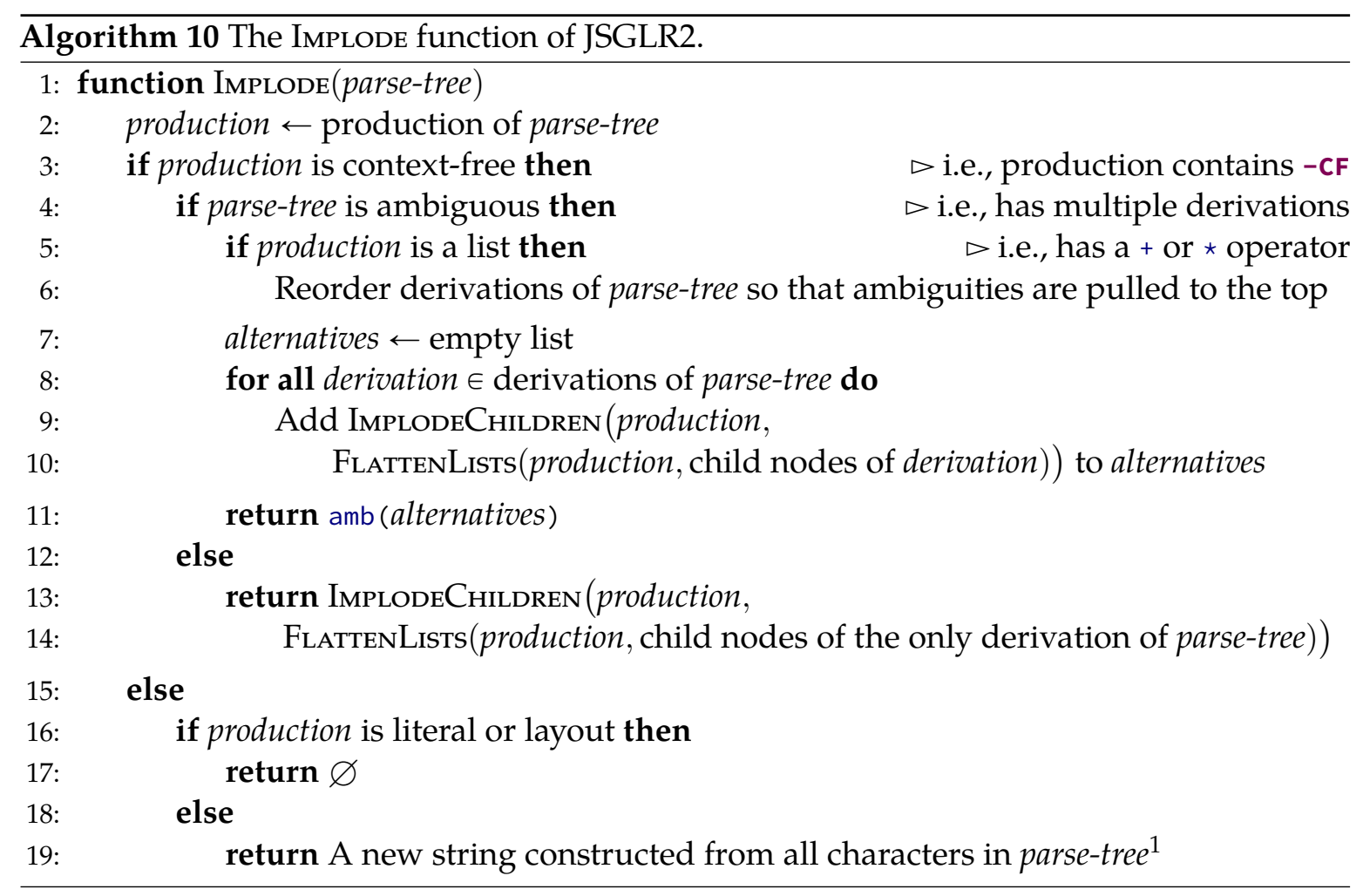


The IMPLODECHILDREN function of Algorithm 11 implodes all children of a parse node. All resulting ASTs that are not empty are added to the list of child-asts. Then, based on the production rule of the parent node, one of the possible types of AST nodes is created: this can be a constructor application, a list, an optional, or a tuple; or, if none of these cases applies, the production must be an injection and the only element of child-asts is returned.

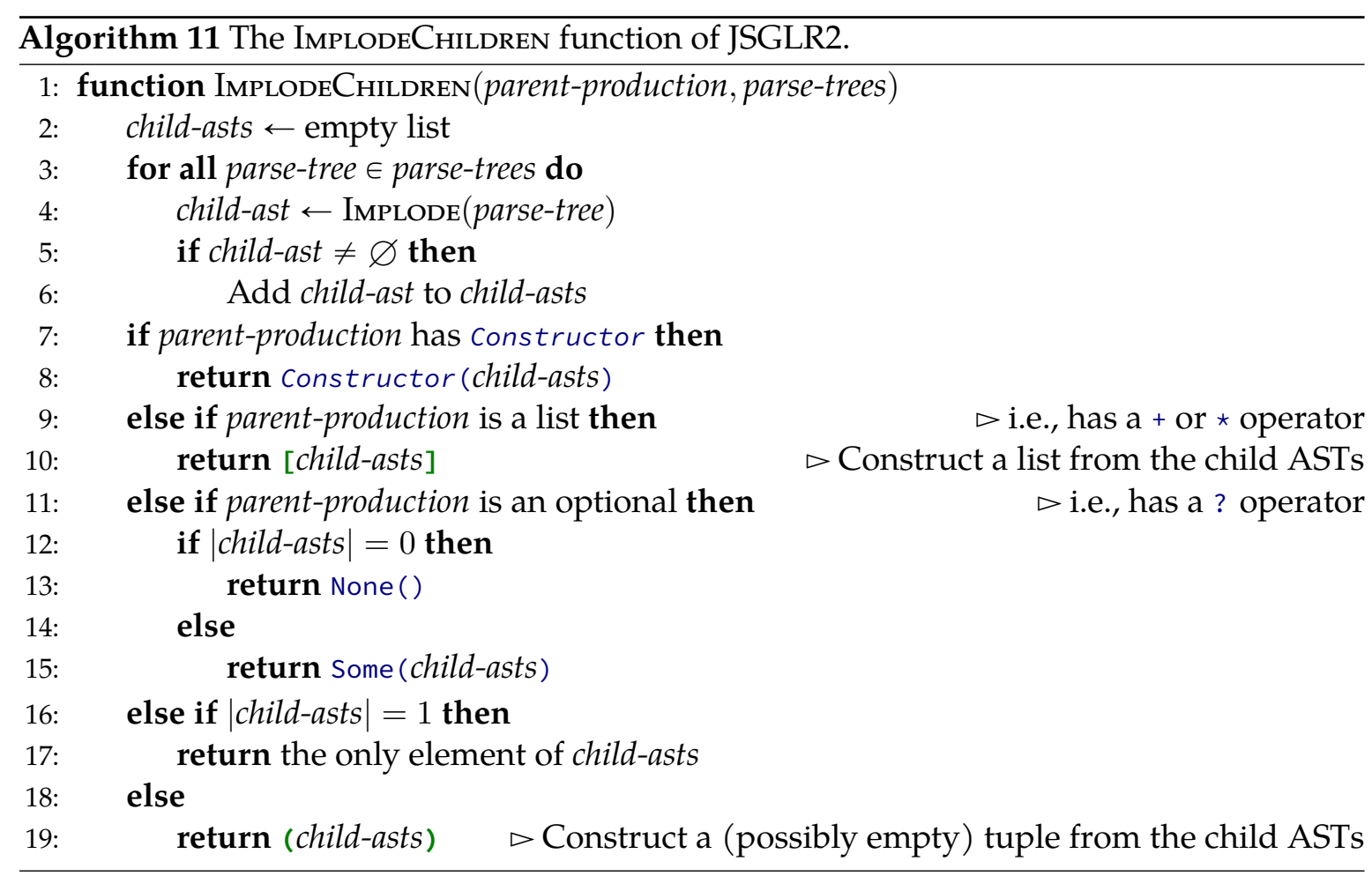

Finally, the FlattenLists function of Algorithm 12 makes sure that the left-recursive lists in the parse tree are flattened out. This function will remove all parse nodes that represent a list production (in SDF3, this is a production rule containing a + or $\star$ ) and return a list of all children of the removed parse nodes. Figure 4.2 shows an example of how this works. If the function receives parse nodes that did not belong to a list production, the original list of parse nodes is simply returned.

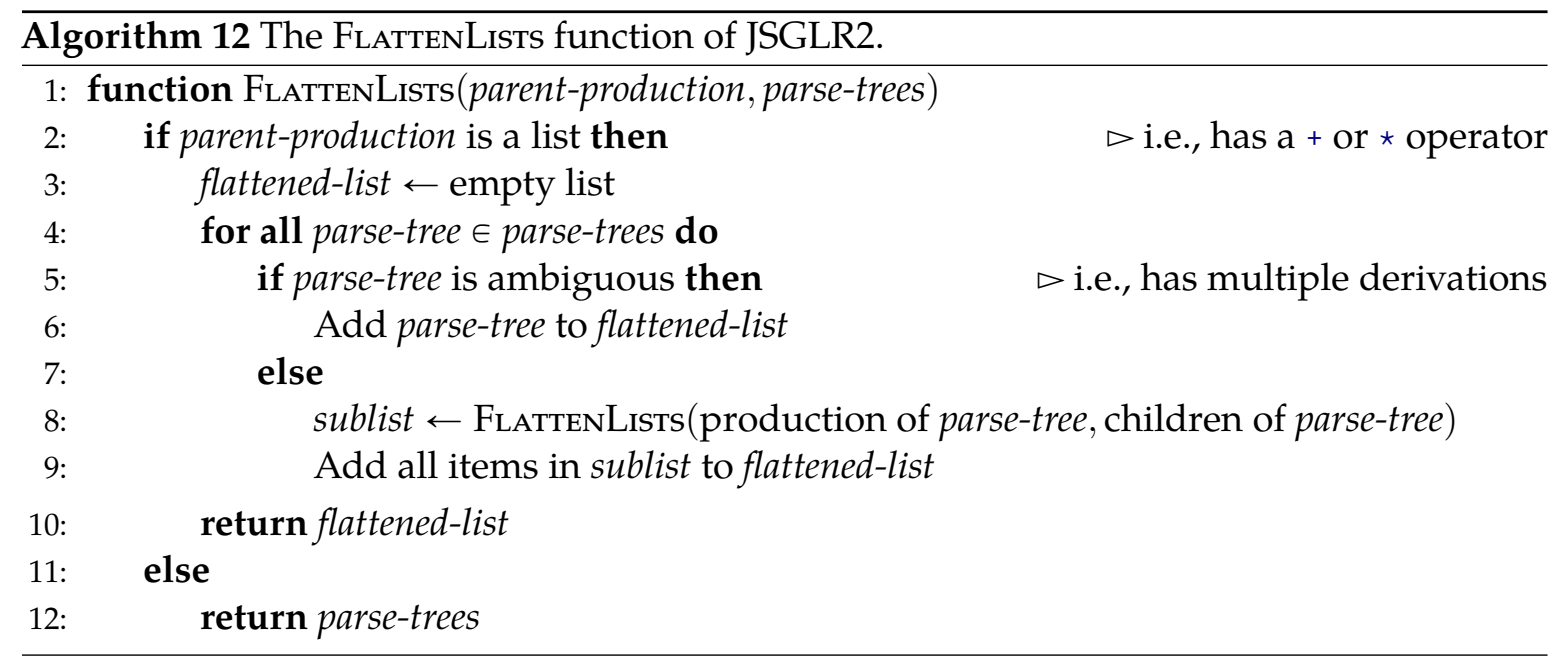

\footnotetext{
${ }^{1}$ In the implementation of the imploder in Java, this string is taken as a substring from the original input string, using an offset that is incremented based on the widths of processed subtrees. This detail is left out to simplify the pseudocode.
} 


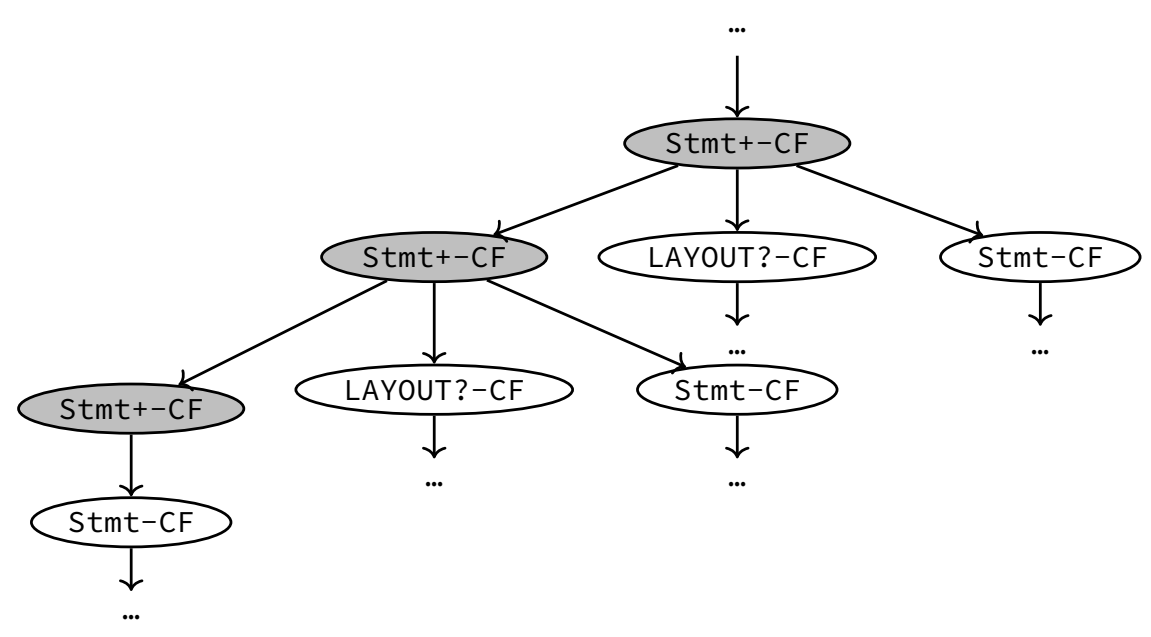

Figure 4.2: An example of the FLATtEnLists function of Algorithm 12. This function takes the parse nodes that represent list productions (the grey nodes) and returns a list containing all their children (the white nodes). In this example, this list thus contains all stmt-cF nodes, interleaved with the LAYOUT?-CF nodes.

\subsubsection{Incremental Imploding}

The incremental imploding algorithm builds upon the non-incremental algorithm outlined in Section 4.1.1. We make two observations that help in the design of the incremental imploding algorithm. Firstly, the result of Algorithm 10 does not depend on the ancestors or siblings of the parse node that is given as input. Secondly, we make use of the fact that parse nodes are immutable, meaning that their descendants cannot change. Therefore, if the imploder receives a parse node as input that has already been imploded before, the previous result can be reused.

The implementation of the incremental imploding algorithm is shown in Algorithm 13. This algorithm builds up a cache, using a dictionary that maps from CST nodes to AST nodes. If the input to the algorithm is already in this dictionary, we can directly return the stored result from it. Else, we call the IMPLODE algorithm from Algorithm 10 and store the result of that in the dictionary before returning it.

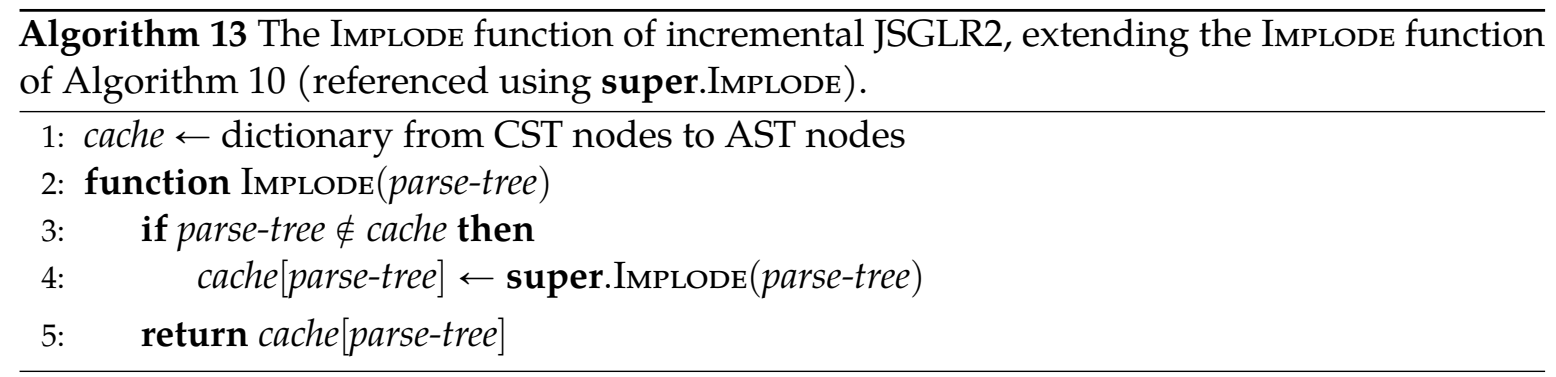

There are two implementation details worth noting about this algorithm. Firstly, because of how Java subclassing and dynamic dispatch works, this new IMPLODE function is executed when IMPLODECHILDREN recursively calls IMPLODE, instead of the original function. Secondly, we implemented the cache dictionary using a WeakHashMap, ${ }^{2}$ allowing the Java garbage collector to delete entries from the dictionary from which the parse nodes no longer exist. Therefore, this cache is not exposed in the interface of the IMPLODE function.

\footnotetext{
${ }^{2}$ https://docs.oracle.com/javase/8/docs/api/java/util/WeakHashMap.html
} 


\subsection{Tokenization}

Most IDEs (Spoofax included) use syntax highlighting to make the code they display easier to read, giving different types of elements in the code different colours. An IDE generates these colours using a list of tokens generated by the tokenizer. The tokenizer creates this list based on the information stored in the CST and the AST, as shown in Figure 4.3. The tokens are similar to those that a lexer would create before parsing token-level grammars, in the sense that they contain information about their location in the input and their type. However, creating the tokens after parsing has the advantage that the token types can be calculated based on their context, making this calculation more precise, especially when grammars of multiple languages are composed together.

\section{offset token kind}

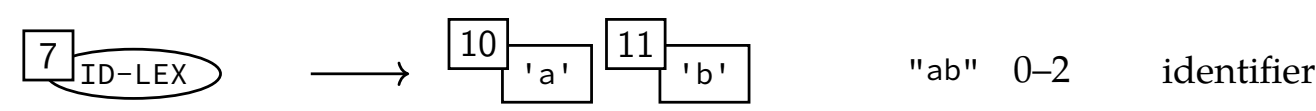

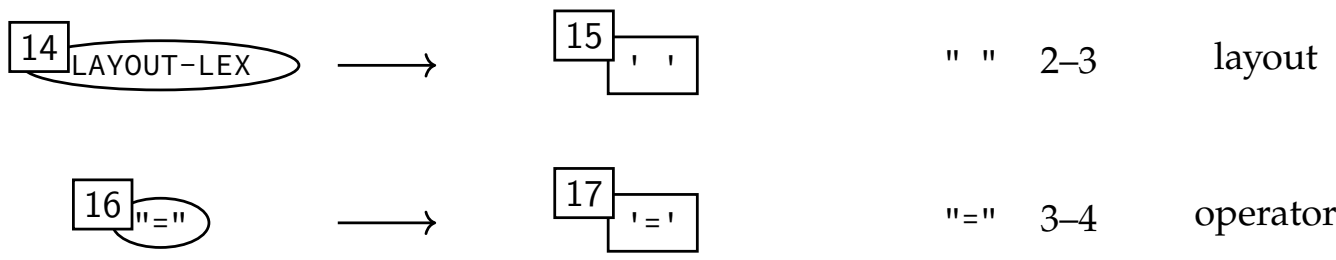

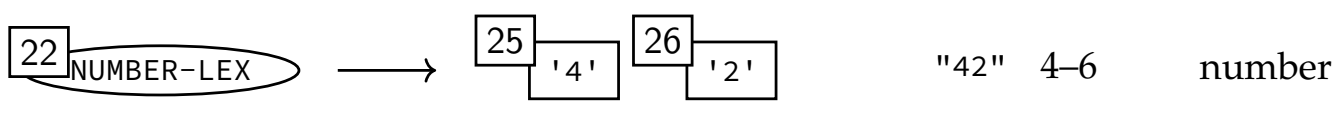

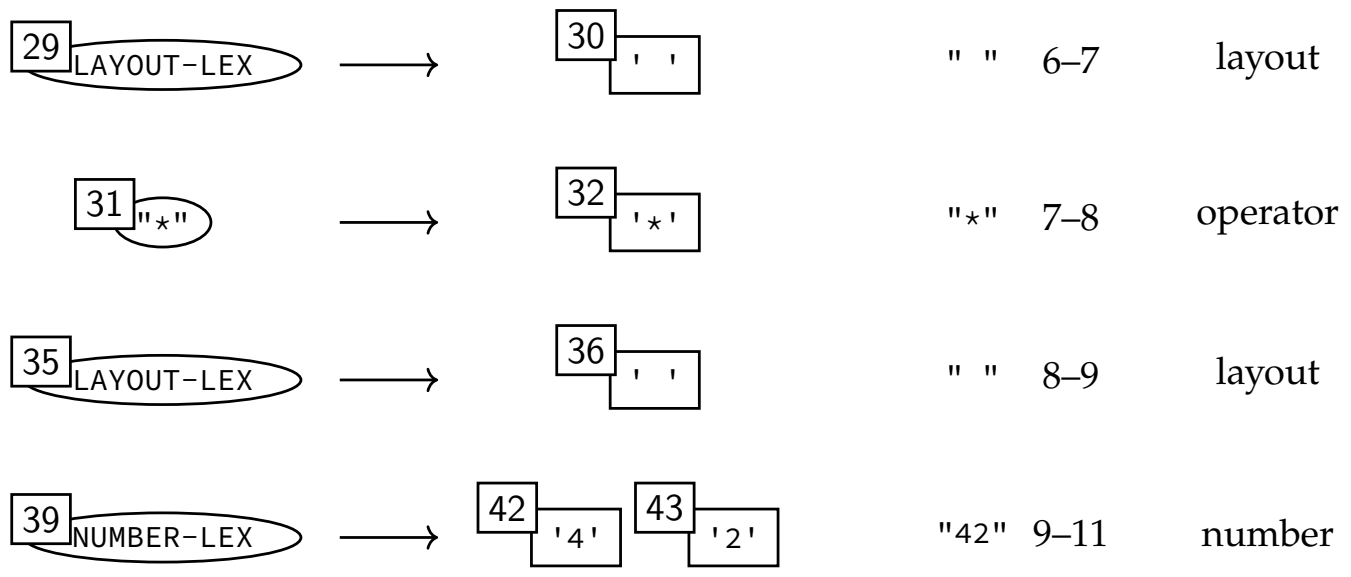

(a) The parse nodes of the CST that the tokenizer generates $\quad$ (b) The resulting tokens. tokens from.

Figure 4.3: The tokens that are generated from the parse tree of Figure 3.2.

An IDE can also use the tokens to generate the locations of error messages. Other parts of the IDE (e.g., the type checker) can report an error or warning by referencing an AST node instead of having to look up the exact position themselves. To accommodate this, the tokenizer attaches two tokens to each node in the AST, indicating the leftmost and rightmost position of the AST in the source code. 


\subsubsection{Tokenizer Algorithm}

The current, non-incremental tokenizer in JSGLR2 works as shown in Algorithms 14 and 15.

The TOKENIZE function of Algorithm 14 generates a list of tokens based on the input parse tree. Just like the imploder, this algorithm recursively generates tokens for all context-free parse nodes.

To make sure that all AST nodes get a left and right token, so-called empty tokens are created for AST nodes that do not correspond to any characters in the input (see line 12 in Algorithm 14 and lines 4-5 in Algorithm 15). These empty tokens span a width of 0 characters but still contain location and production information.

The tokenizer also keeps track of the positions of tokens. Positions of characters are composed of three integers: offset, the zero-based index of a character in the entire input; line, the one-based index of the line that the character appears on; and column, the one-based column index of the character on its line. ${ }^{3}$ The begin position of a token is the position of the first character of this token, while its end position is the position after the last character of the token. This means that for empty tokens, their begin position is equal to their end position.

The initial value for the start-position parameter is a position with offset 0 , line 1 , and column 1 . While iterating over the child trees, the position is advanced based on the position ranges of the subtrees (see line 11 in Algorithm 14 and line 2 in Algorithm 15).

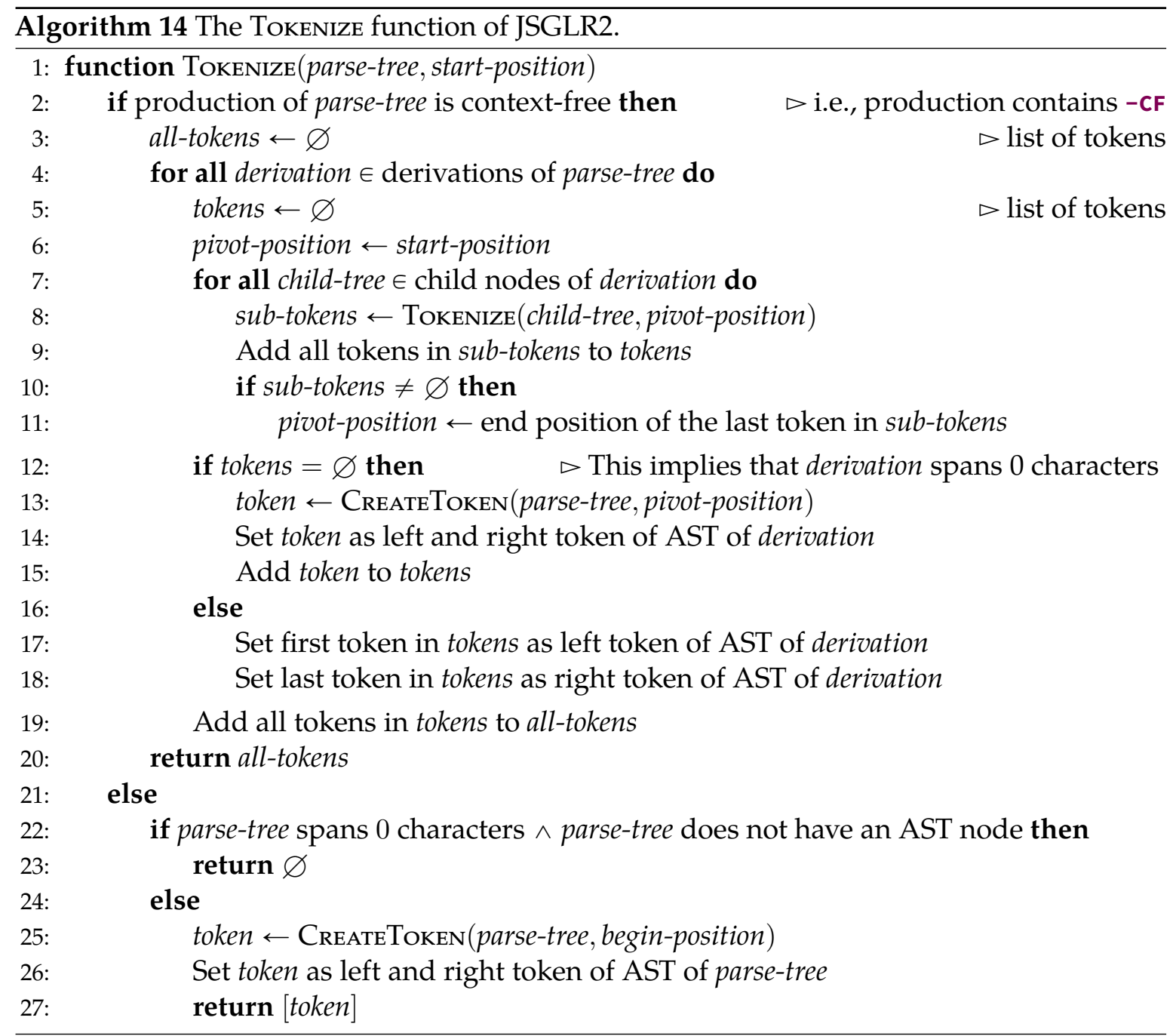

\footnotetext{
${ }^{3}$ The pseudocode does not show this level of detail to make it more readable. We assume that the + operator can add two position(-range)s together.
} 


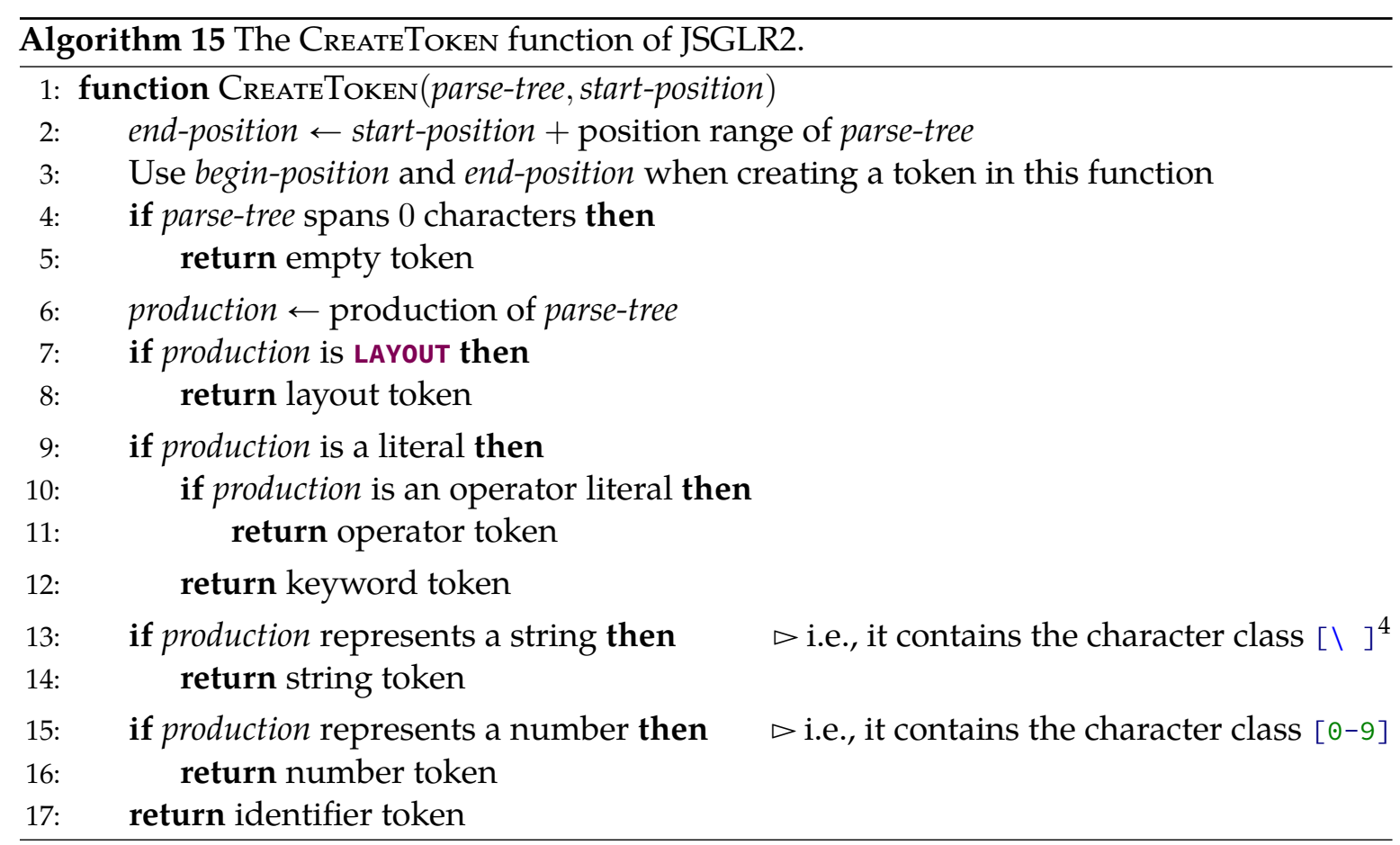

\subsubsection{Incremental Tokenization}

There is one main challenge related to incremental tokenization: storing the absolute positions of tokens would require updates in many tokens for even a small update in the input. As an example, when the input changes from "ab $=42 \star 42$ " to "ans $=42 \star 42$ ", an incremental tokenizer does not only need to replace the first token (representing "ab") by a new token (representing "ans"), but it would also need to increment the positions of all following tokens by one.

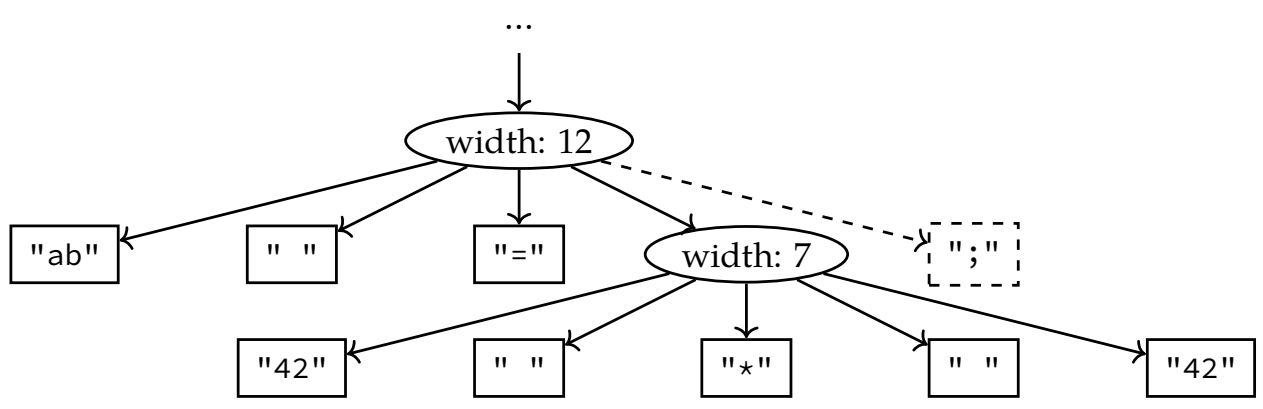

Figure 4.4: An example token tree, generated from the parse tree of Figure 3.2. An imaginary semicolon has been added for illustration purposes.

To solve this challenge, we turn the list of tokens into a tree-shaped data structure, with the tokens as leaf nodes. In this tree, every node stores its width in terms of the number of characters, the number of lines, and the number of columns in the last line. Absolute positions of tokens can then be calculated on the fly by summing up the widths of all siblings to the left along the spine of a token to the root. An example of such a token tree is shown in Figure 4.4. When calculating the start offset of the token "*", we sum up the widths of its left siblings $(2+1=3)$ and the left siblings of its parent node $(2+1+1=4)$, arriving at

\footnotetext{
${ }^{4}$ If the right-hand side of the production rule contains the space character (and it is not layout), it must be enquoted in some way, so it represents a string in its language.
} 
a start offset of 7 . When calculating the start offset of the imaginary "; " token, summing up the widths of its left siblings gives a start offset of $2+1+1+7=11$.

Using a tree-shaped data structure to store the tokens has the advantage that we can make the tokenizer incremental similar to the incremental IMPLODE function, as shown in the TREEToKEnIze function in Algorithm 16. If the tokenizer has already processed a parse tree, it can reuse that result from the cache. Note that the same implementation details apply as for the incremental IMPLODE function in Algorithm 13.

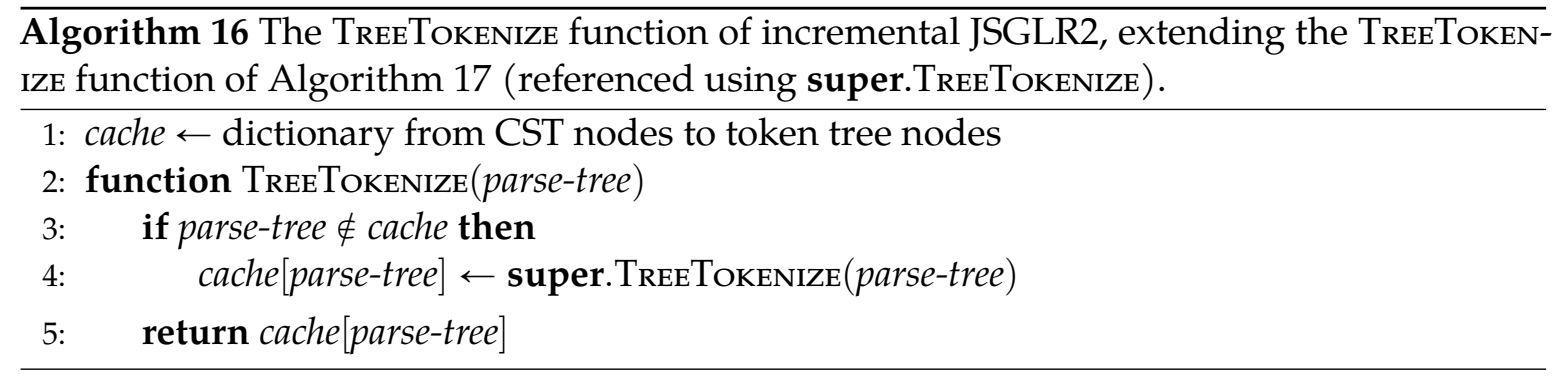

The non-incremental TreeToKenize function in Algorithm 17 works roughly the same as the non-incremental ToKenize function in Algorithm 14. The main difference is that the TREeToKenize function does not return a list of tokens but a token tree node. Each token tree node stores its width (equal to the sum of the widths of its children), its left- and rightmost tokens, and a reference to its parent.

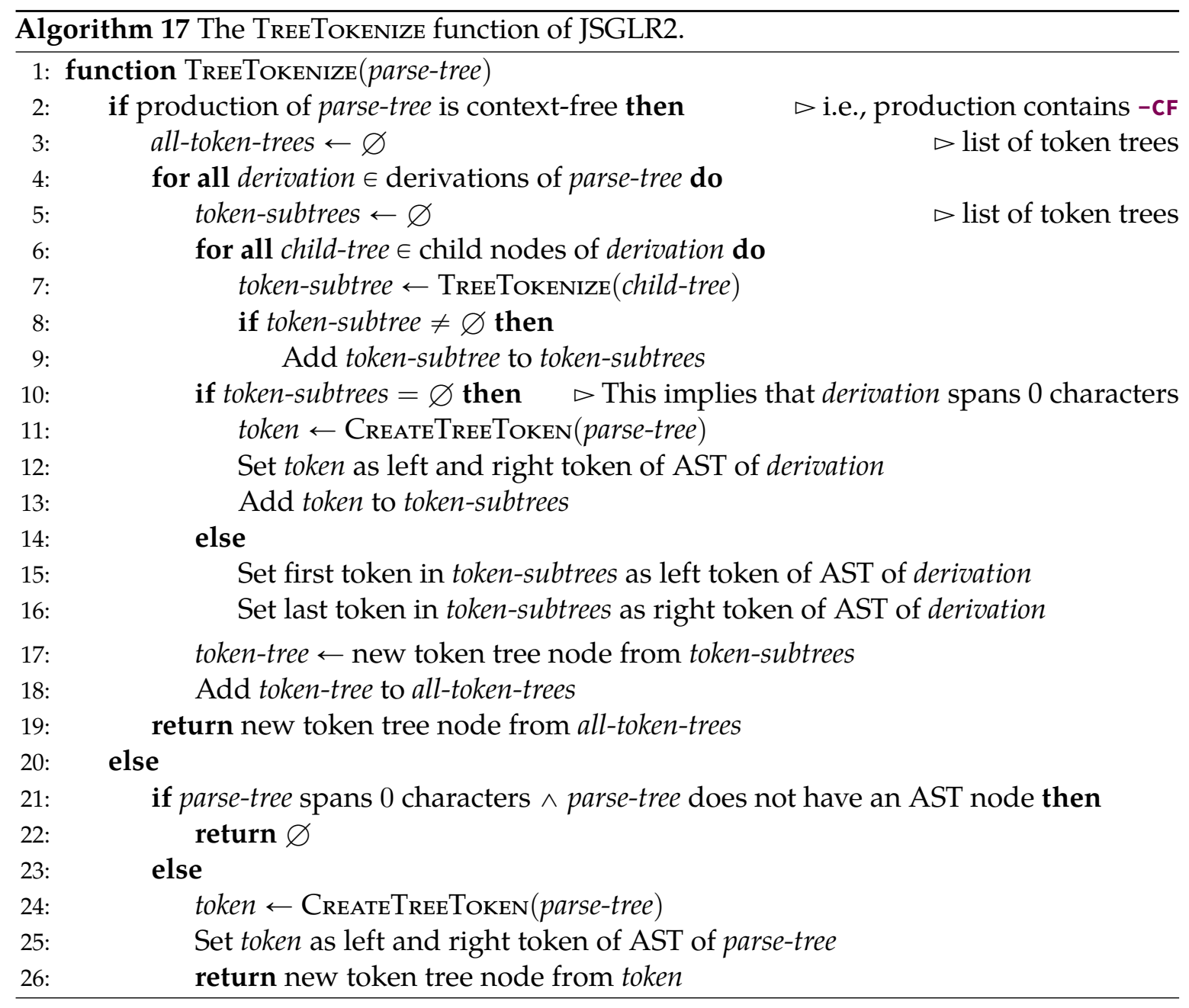


The CREATETREeToKen function in Algorithm 18 has one main difference compared to the creation of traditional tokens in the CREATEToKen function in Algorithm 15: instead of storing a begin- and end-position, a token in the token tree only stores its width. ${ }^{5}$

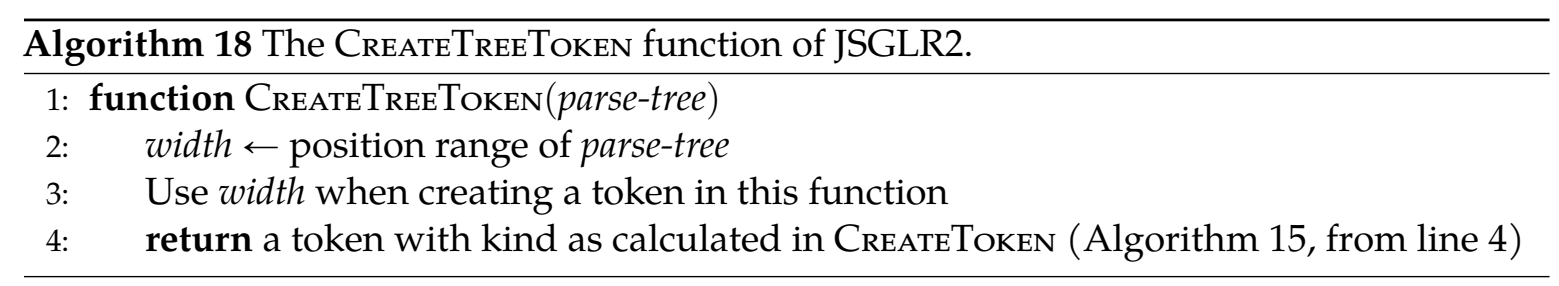

\footnotetext{
${ }^{5}$ Just like how the Java implementation of the imploder needs the original input string to do its calculations more efficiently (see footnote 1), the same goes for calculating the width of a tree token. Ironically, this calculation also requires knowing the absolute position of a token in its original input string, so the Java implementation of the tokenizer needs to keep track of this. However, since the tree tokens do not store their absolute position directly, this does not affect the incrementality of the algorithm.
} 
This page is intentionally left blank. 


\section{Chapter 5}

\section{Performance Evaluation}

We evaluate the ISGLR parser in two different ways: measurements and benchmarks. All evaluations use software repositories on $\mathrm{GitHub}^{1}$ as input to the parser, making use of the recorded history of the files. We describe the evaluation setup and corpus in Section 5.1.

We measure statistics for certain events happening during parsing and the parse nodes in the resulting parse forest in Section 5.2. We find that irreusable parse nodes make up $27 \%$ of the average parse forest, which causes the majority of the breakdown events during incremental parsing. Languages like Java and WebDSL, which use fence characters like curly brackets and semicolons, have less irreusable parse nodes in their parse forests and have more parse node reuse than a language like SDF3, which does not have such fences. The majority of the irreusable parse nodes is not exposed on top of the input stack during a subsequent incremental parse, so on average, the ISGLR parser can reuse $99 \%$ of a previous parse tree.

In Sections 5.3 and 5.4, we evaluate the run time and memory performance of the ISGLR parser, respectively. We consider both the scenario of batch parses and the scenario of incremental parses. In batch mode, we compare the performance of the ISGLR parser with two existing JSGLR2 parser variants: the Standard parser and the Elkhound variant (see Section 2.4.3). The run time/memory performance of the ISGLR parser in batch mode is $24 \%$ slower $/ 23 \%$ higher than the Standard parser and $60 \%$ slower $/ 68 \%$ higher than the Elkhound variant. In incremental mode, for changes that are smaller than $1 \%$ of the input size on average, the run time performance of the ISGLR parser is $9 \times$ faster than the Standard parser in batch mode.

In Section 5.5, we discuss possible threats to the validity of this evaluation and to what extent we countered them.

\subsection{Setup}

We executed the evaluation on a server machine with two 32-core AMD EPYC processors with a base frequency of $2.3 \mathrm{GHz}$ and 256 GB RAM provided by sixteen DDR4-2933 modules. The used operating system is Ubuntu 20.04 (kernel version 5.4.0-77), using OpenJDK version 1.8.0_275-b01 and Apache Maven 3.6.3.

We ran a series of evaluation scripts, written in Scala 2.13 unless noted otherwise, inside a Docker container ${ }^{2}$ using Docker version 20.10.7. The scripts are configurable using a YAML ${ }^{3}$ file describing the list of languages and sources to evaluate the parser with.

\footnotetext{
${ }^{1}$ https://github.com/

${ }^{2}$ The Docker container, including the used scripts, are publicly available on GitHub: https://github.com/metaborg/jsglr2evaluation

${ }^{3}$ https://yaml.org/
} 
We separated the evaluation scripts into the following steps:

Pull Languages Pull the latest version of the Spoofax languages used for evaluation from GitHub and build these projects using Maven.

Pull Sources Pull the latest versions of the sources in the evaluation corpus from GitHub. This only pulls versions from Git commits that have changes in source files with the file extension that belongs to the language, e.g., . j ava for the Java language.

Preprocessing Validate the evaluation corpus in several ways, see below.

Measurements Perform measurements while parsing and compare parse forests of subsequent versions, see below.

Time Benchmarks Execute benchmarks that measure the run time of the parsers, see below.

Memory Benchmarks Execute benchmarks that measure the memory usage and cache size of the parsers, see below.

Post-Processing Process the Comma-Separated Values (CSV) files that resulted from the various benchmarks, add extra data like file sizes and change sizes, and combine all this into larger CSV files.

Generate Figures Generate plots with Matplotlib, ${ }^{4}$ in Python 3.8.5.

Publish Generate a web page from the evaluation results and publish it to the website https://www.spoofax.dev/jsglr2evaluation-site/.

Preprocessing We validate the evaluation corpus in several ways.

For the batch scenario, we ensure the validity of inputs and parsers by parsing all inputs with all parser variants. Some files can be invalid due to, for example, a mismatch between language grammar versions and are automatically removed from the batch scenario corpus. We inspect those files manually to judge whether they are actually invalid or that the language grammar was incorrect. To validate consistency between the JSGLR2 variants, we check that all variants produce the same AST in batch mode.

For the incremental scenario, we validate that an incremental parse of a file has the same result as a batch parse of the same file. Naturally, this is only done for files that have a previous version in the evaluation corpus. If this validation fails, it would mean that the ISGLR parser is incorrect and the entire evaluation is aborted.

Measurements We performed measurements while parsing the input from the evaluation corpus. We counted the number of parse nodes in the resulting parse forests, recording their type (parse node or character node) and whether they are irreusable or not.

We also compared parse forests between subsequent versions. During an incremental parse, we count how many parse nodes are reused or broken down. We also count the number of parse nodes that are rebuilt, i.e., that were broken down during parsing, but were recreated with the exact same children as in the parse forest of the previous version. Of the parse nodes that are broken down, we record the reason for the breakdown.

Time Benchmarks The benchmarks that measure the run time of the parsers make use of the Java Microbenchmark Harness $(\mathrm{JMH}){ }^{5}$ version 1.25.2. The setup phase of a JMH benchmark initializes the parse table and parser for the given language. Also, when doing a benchmark of an incremental parse, the setup phase parses the first version of the input to populate the cache.

\footnotetext{
${ }^{4}$ https: //matplotlib.org/

${ }^{5}$ https://github.com/openjdk/jmh
} 
JMH runs a predefined action in multiple so-called iterations, where each iteration runs the action as often as possible for 10 seconds. We run the time benchmarks using 10 warmup iterations and 10 regular iterations. These warmup iterations execute the action but do not measure its run time, to warm up the Java Virtual Machine (JVM).

We separated the time benchmarks into multiple executions of JMH, where each of these benchmark runs considers two consecutive versions of the evaluation corpus. In the setup phase of the benchmark, we save the results of the first version (parse forests, ASTs, tokens). We defined the benchmark action as a call to the parser with the second version as input, using the results of the first version for incrementality.

Memory Benchmarks We run two kinds of memory benchmarks: one that measures the allocations during parsing and one that calculates the cache size after a parser saves a result to the cache. For every language, the benchmark script sampled 100 files from the evaluation corpus to run the memory benchmarks with.

We measure memory allocations by instrumenting the JVM to count every object constructor called during parsing, using a custom allocation instrumenter library ${ }^{6}$ forked from Google. This library instruments all constructors of all loaded Java classes with a call to an observable. To this observable, we attach an observer that sums the sizes of all objects that are created during parsing.

We measure the cache size using the Java Object Layout library. ${ }^{7}$ This library can calculate the size of an object, including the size of all of its references, recursively. For every file in the benchmark, we subtract a measurement taken before parsing from a measurement taken after parsing to obtain the impact on the memory usage of the cache for that file.

\subsubsection{Evaluation Corpus}

Table 5.1 shows the languages and sources that we used to evaluate the performance of the ISGLR parser. We chose three languages with different characteristics: Java as a GeneralPurpose Language (GPL), SDF3 as Domain-Specific Language (DSL) that is used as a metalanguage in Spoofax, and WebDSL as DSL that is used to generate websites. The grammars of these languages are publicly available on GitHub.

Table 5.1: Corpus used to evaluate the performance of the ISGLR parser. The numbers of files/lines and the (file/change) sizes are the average of all versions. The change sizes are equal to the number of removed characters plus the number of added characters.

\begin{tabular}{l|l|r|r|r|r|r|r} 
Language & Source & Versions & Files & Lines & Size (B) & $\begin{array}{c}\text { Mean file } \\
\text { size (B) }\end{array}$ & $\begin{array}{c}\text { Change } \\
\text { size (B) }\end{array}$ \\
\hline \multirow{3}{*}{ Java } & StringUtils & 16 & 1 & 9635 & 396297 & 396297 & 850 \\
& gson & 16 & 204 & 37163 & 1265001 & 6189 & 988 \\
& slf4j & 16 & 238 & 26632 & 893078 & 3756 & 8606 \\
\hline \multirow{3}{*}{ WebDSL } & builtin.app & 10 & 1 & 3403 & 98147 & 98147 & 650 \\
& YellowGrass & 16 & 53 & 6019 & 166767 & 3171 & 1168 \\
& elib-utils & 16 & 17 & 1422 & 39480 & 2322 & 172 \\
\hline \multirow{5}{*}{ SDF3 } & NaBL & 16 & 126 & 4386 & 98690 & 781 & 606 \\
& DynSem & 16 & 4 & 437 & 9716 & 2429 & 130 \\
& FlowSpec & 16 & 15 & 567 & 12209 & 816 & 516 \\
& Stratego & 7 & 64 & 2950 & 79224 & 1240 & 379 \\
& WebDSL & 16 & 26 & 3252 & 86032 & 3308 & 114
\end{tabular}

\footnotetext{
${ }^{6}$ https://github.com/mpsijm/simple-allocation-instrumenter

${ }^{7}$ https://github.com/openjdk/jol
} 
For two sources, we picked a single large file to investigate the effect of this on the performance of the parser. For Java, we chose the file stringutils.java, a file of almost $400 \mathrm{kB}$ from Apache's Commons Lang library. For WebDSL, we chose the file builtin.app, a file of almost $100 \mathrm{kB}$ from the WebDSL compiler project. For other sources, we picked entire repositories. All sources are publicly available on GitHub.

\subsection{Measurements Results}

In this section, we will discuss measurements about the parse nodes in the parse forest returned as result from the parser (Section 5.2.1) and the reasons for breaking down parse nodes (Section 5.2.2). Table 5.2 displays the most interesting results from the measurements, as an average of the measurements over all corpus sources of a language. See Appendix A.1 for the full measurement results.

Table 5.2: Measurements for parse nodes and breakdowns for the different languages in the evaluation corpus as shown in Table 5.1.

\begin{tabular}{c|c|c|c|c|c|c|c|c} 
& \multicolumn{3}{|c|}{ Parse nodes (\% of total nodes) } & \multicolumn{3}{c}{ Breakdowns $\%$ of total breakdowns) } \\
Language & $\begin{array}{c}\text { Irre- } \\
\text { usable }\end{array}$ & Reused & $\begin{array}{c}\text { Broken } \\
\text { down }\end{array}$ & Rebuilt & $\begin{array}{c}\text { Contains } \\
\text { Change }\end{array}$ & $\begin{array}{c}\text { Irre- } \\
\text { usable }\end{array}$ & $\begin{array}{c}\text { No } \\
\text { actions }\end{array}$ & $\begin{array}{c}\text { Wrong } \\
\text { state }\end{array}$ \\
\hline Average & $27.25 \%$ & $99.12 \%$ & $0.67 \%$ & $0.48 \%$ & $40.68 \%$ & $56.23 \%$ & $0.00 \%$ & $3.08 \%$ \\
\hline Java & $19.08 \%$ & $99.60 \%$ & $0.17 \%$ & $0.08 \%$ & $51.35 \%$ & $46.67 \%$ & $0.00 \%$ & $1.98 \%$ \\
WebDSL & $21.32 \%$ & $99.55 \%$ & $0.28 \%$ & $0.15 \%$ & $51.35 \%$ & $43.76 \%$ & $0.00 \%$ & $4.89 \%$ \\
SDF3 & $41.36 \%$ & $98.21 \%$ & $1.57 \%$ & $1.21 \%$ & $19.35 \%$ & $78.28 \%$ & $0.00 \%$ & $2.38 \%$
\end{tabular}

\subsubsection{Parse Nodes}

The left part of Table 5.2 shows four measurements as a percentage of the total number of parse nodes:

Irreusable is the percentage of parse nodes that is marked as irreusable because they were created while the parser was parsing non-deterministically.

Reused is the percentage of parse nodes that are reused during an incremental parse, i.e., parse nodes that the parser could shift directly together with all its descendent nodes.

Broken down is the percentage of parse nodes that the parser has to break down during an incremental parse.

Rebuilt is the percentage of parse nodes that are created during an incremental parse for the same production rule and with the same children as in the previous parse.

Note that the percentages "Reused" and "Broken down" do not fully sum up to $100 \%$ for any language, while intuitively, a parse node is either reused or broken down. However, the CнескUрDates procedure might skip larger parse nodes, as shown in line 8 of Algorithm 3. Any descendants of these skipped parse nodes are not counted in the "Broken down" percentage, because the parser does not actively break them down.

Java and WebDSL have over $99.5 \%$ parse node reuse, but SDF3 has only $98 \%$ parse node reuse. Similarly, parse forests for Java and WebDSL have around 20\% irreusable parse nodes, while in parse forests for SDF3 they make up $41 \%$ of all parse nodes. After manually inspecting parse forests for the different languages, we found that the higher number of irreusable parse nodes for SDF3 can be explained by the lack of fencing characters in the language. 
Languages like Java and WebDSL use curly brackets (\{\}) and semicolons (;) as fencing characters. Curly brackets indicate the nesting of parts of code, for example, the body of a function or the body of an if-statement. Similarly, semicolons terminate a statement within a block of code. However, in SDF3, code sections are bounded using keywords (like context-free syntax) and definitions of production rules do not have a terminating character. The parser will need to parse any word non-deterministically, as discussed in Section 3.2.2: a word can either be the next sort in the right-hand side of a production rule, the sort on the left-hand side of a new production rule, or a keyword that indicates the start of a new code section. Therefore, the parse nodes which correspond to code sections and production rules are marked as irreusable, which makes the ISGLR parser break down many of these parse nodes during an incremental parse when they end up at the top of the input stack. In languages that do use fencing characters, this does not happen, because the fences consist of a single character: when the parser encounters a fence, there is only one way of parsing the statement (block) that has this fence as the final character.

Of the parse nodes that are broken down, on average, $71 \%$ of them are rebuilt. ${ }^{8}$ This implies that the majority of the breakdowns are unnecessary in hindsight. However, because of the non-deterministic nature of scannerless parsing, these breakdowns are still necessary during parsing since the parser can only look one character ahead. Still, for changes that are smaller than $1 \%$ of the input size on average, we conclude that a reuse of $99 \%$ on average is acceptable for an incremental parser.

\subsubsection{Breakdowns}

The right part of Table 5.2 shows four measurements as a percentage of the total number of broken-down parse nodes:

Contains Change is the percentage of breakdowns that happen when that parse node contains a change as calculated by the diff.

Irreusable is the percentage of breakdowns that break down an irreusable parse node.

No actions is the percentage of breakdowns occurring when there are no valid actions in the parse table.

Wrong state is the percentage of breakdowns that break down a parse node because the state matching test failed, i.e., the parse state reference stored in the parse node is not the same as the current parse state.

The number of breakdowns caused by changed parse nodes is $41 \%$ on average. All other broken-down parse nodes are not located in the spine between the updated character nodes and the root of the parse forest. Ideally, an incremental parser would only break down parse nodes that are located in this spine, including the parse nodes that cannot be reused because the state matching test failed. In the evaluated corpus, another $3 \%$ of parse nodes fall in this latter category.

However, most of the breakdowns, 56\% on average, are caused by irreusable parse nodes. This can be explained by the fact that a quarter of all parse nodes is irreusable, so many of them end up exposed at the top of the input stack, causing them to be broken down. This effect is stronger in the parsing of SDF3 than for the parsing of Java or WebDSL, for the same reasons as mentioned in Section 5.2.1.

None of the breakdowns is caused by the absence of valid actions in the parse table. This scenario does not occur in this evaluation, since we only consider input files that are valid according to the grammar of the language.

\footnotetext{
${ }^{8}$ This value is calculated from Table 5.2 by dividing the value in the "Rebuilt" column by the value in the "Broken down" column.
} 


\subsection{Time Benchmark Results}

This section shows the performance results of the ISGLR parser in terms of run time, compared to other parser variants in JSGLR2 and Tree-sitter (Brunsfeld 2018). Tree-sitter is a parser based on the Incremental LR (ILR) parsing algorithm by Wagner and Graham (1998). We will describe Tree-sitter in more detail in Section 6.1.1.

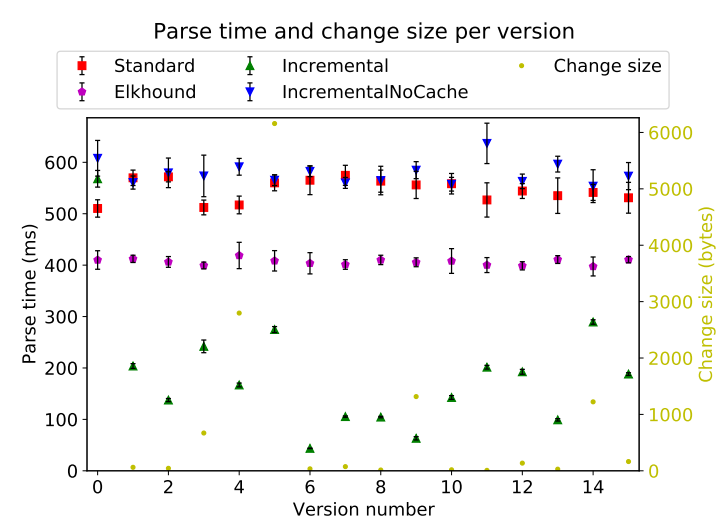

(a) Parse times of Stringutils.java from Apache's commons-lang library.

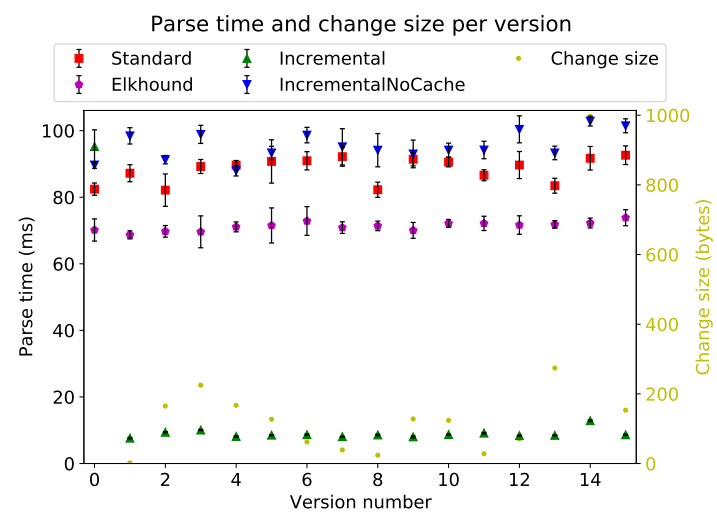

(c) Parse times of the elib-utils repository.

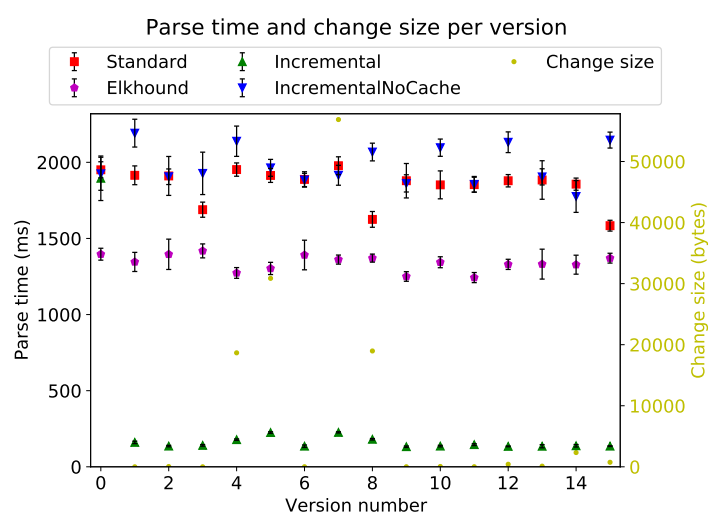

(b) Parse times of the $s l f 4 j$ repository.

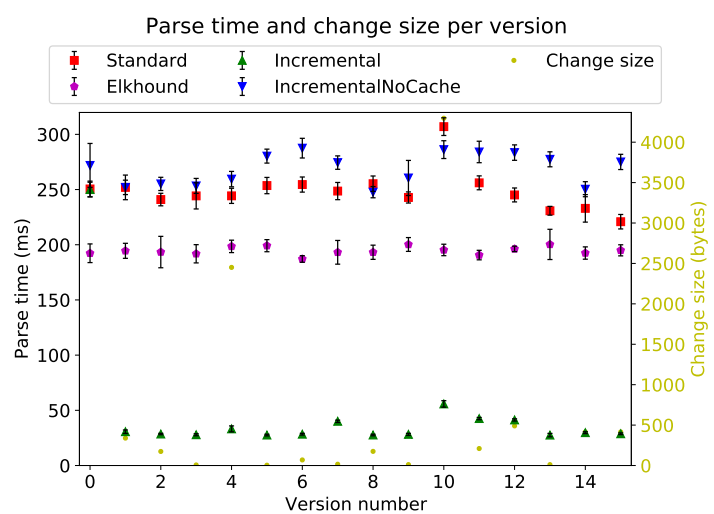

(d) Parse times of the nabl repository.

Figure 5.3: Parse times of the last 16 versions of several files and repositories from GitHub, excluding imploding and tokenization. The red squares represent the Standard JSGLR2 parser, the purple pentagons represent the Elkhound parser variant, the blue downwardpointing triangles represent the incremental parser in batch mode (i.e., without cache), and the green upward-pointing triangles represent the incremental parser in incremental mode. The yellow dots indicate the size of the changes in a particular version, which is the sum of the number of removed and inserted characters, on the second y-axis.

Table 5.4: Average parse times for the different languages in the evaluation corpus, as shown in Table 5.1, for three JSGLR2 parser variants. The values indicate the parse time in milliseconds, excluding imploding and tokenization.

\begin{tabular}{c|r|r|r|r} 
Language & Standard & Elkhound & $\begin{array}{r}\text { Incremental } \\
\text { (no cache) }\end{array}$ & Incremental \\
\hline Average & 785.510 & 597.235 & 839.299 & 82.503 \\
\hline Java & 1974.375 & 1497.341 & 2112.104 & 203.043 \\
WebDSL & 233.029 & 175.540 & 244.903 & 23.681 \\
SDF3 & 149.127 & 118.824 & 160.888 & 20.784
\end{tabular}


All benchmark results in this section show the average run time performance over 10 benchmark iterations, where the error bars in the plots indicate the $99 \%$-confidence interval. The full time benchmark results are shown in Appendix A.2.

We evaluate the run time performance separately with and without the imploding and tokenization steps, as shown in the bottom row of Figures 2.10 and 3.12. The Tree-sitter parser is only included in the second comparison, where for both parsers their full parsing pipeline is timed from start to end.

Parsing Only Figure 5.3 shows the parse times for four of the sources in the evaluation corpus, where the ISGLR parser is measured both in batch mode and in incremental mode. The parse times in this figure are measured for only parsing, i.e., excluding imploding and tokenization. The average parse times for the three languages of the evaluation corpus (Section 5.1.1) are shown in Table 5.4.

The overall result is that the ISGLR parser in incremental mode has a significant speedup compared to the Standard parser in batch mode of $9.5 \times$, with changes that are smaller than $1 \%$ of the input size, on average. The ISGLR parser in batch mode is $7 \%$ slower than the Standard JSGLR2 parser variant, and 41\% slower than the Elkhound variant.

Interestingly, Figure 5.3(a) shows some outliers where the ISGLR parser is significantly slower to parse incremental updates to stringutils.java than for the other sources of the evaluation corpus. Remember from Section 5.1.1 that Stringutils. java is a Java file of almost $400 \mathrm{kB}$. Some of the slower incremental parsing times can be attributed to the size of the diff, like version 5 . However, version 11 has only added eight characters with respect to version 10 , yet the incremental parsing time is one of the longest for this figure. Specifically, these eight characters were added in four different places throughout the file. Further inspection using a Java profiler ${ }^{9}$ showed that executing the diff algorithm took $7 \times$ more time than the incremental parsing for this particular diff. We consider optimizing the diff algorithm out of scope for this thesis.

With Imploding And Tokenization Figure 5.5 shows the parse times for four of the sources in the evaluation corpus. The parse times of the JSGLR2 parser variants are measured for the full JSGLR2 parsing pipeline, which includes imploding and tokenization. The average parse times for the three languages of the evaluation corpus (Section 5.1.1) are shown in Table 5.6. The parse times for Tree-sitter (Brunsfeld 2018) are only measured for the Java language since no Tree-sitter grammars for WebDSL and SDF3 are available.

The overall result is that the ISGLR parser in incremental mode has a significant speedup compared to the Standard parser in batch mode of $9.0 \times$, with changes that are smaller than $1 \%$ of the input size, on average. The ISGLR parser in batch mode is $24 \%$ slower than the Standard JSGLR2 parser variant, and 60\% slower than the Elkhound variant. Comparing this with the results of Table 5.4, we see that the parsing phase takes up $97 \%$ and $95 \%$ of the full JSGLR parsing pipeline for the Standard and Elkhound parser variants, respectively. For the ISGLR parser, this percentage drops to $83 \%$ in batch mode and $91 \%$ in incremental mode. This indicates that the incremental imploding and tokenization algorithms have more overhead compared to the incremental parsing algorithm than the original algorithms.

The ISGLR parser is roughly $11 \times$ slower than the Tree-sitter parser, both in batch mode and in incremental mode. It is almost impossible to conclude anything from this because Tree-sitter is written in the C programming language, while JSGLR2 is written in Java. Generally speaking, a program written in Java is slower than the same program written in C, but the slowdown factor varies heavily depending on the type of program (Fourment and Gillings 2008). However, the speedup of incremental parsing compared to batch parsing for Tree-sitter is similar, although slightly better $(12 \times)$ compared to the ISGLR parser.

\footnotetext{
${ }^{9}$ https://www.ej-technologies.com/products/jprofiler/overview.html
} 


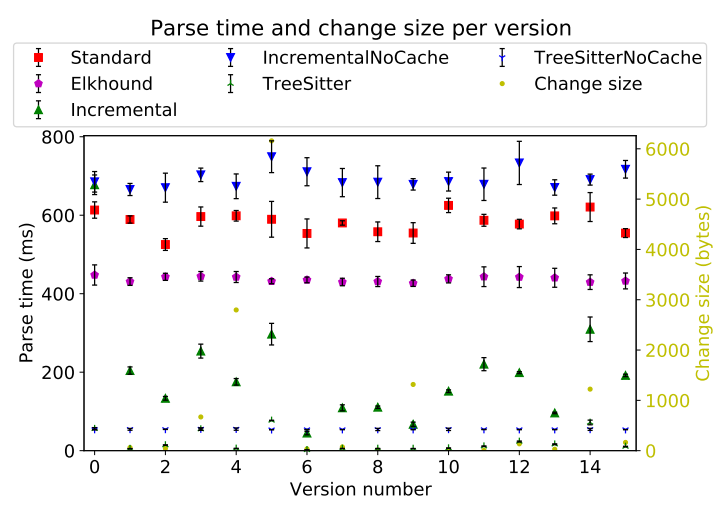

(a) Parse times of Stringutils.java from Apache's commons-lang library.

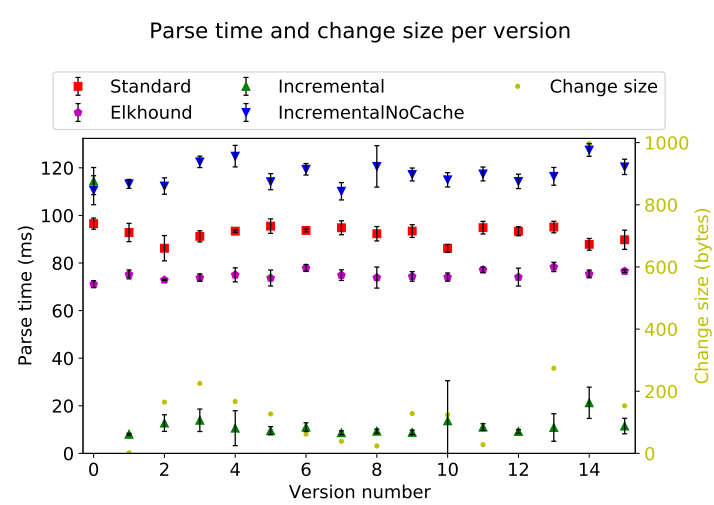

(c) Parse times of the elib-utils repository.

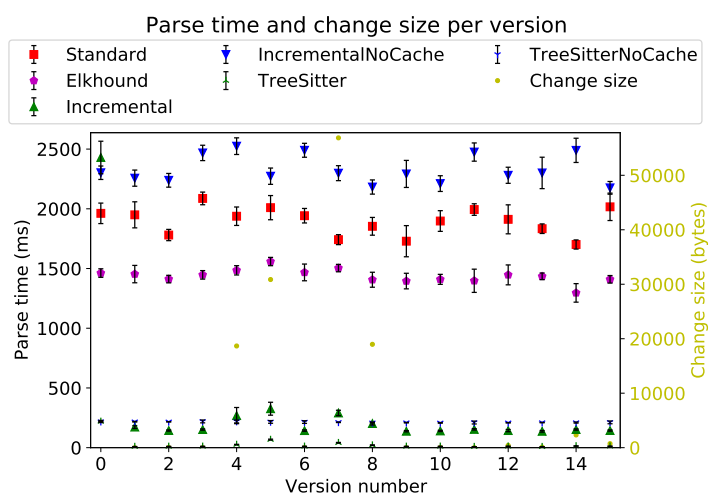

(b) Parse times of the $s l f 4 j$ repository.

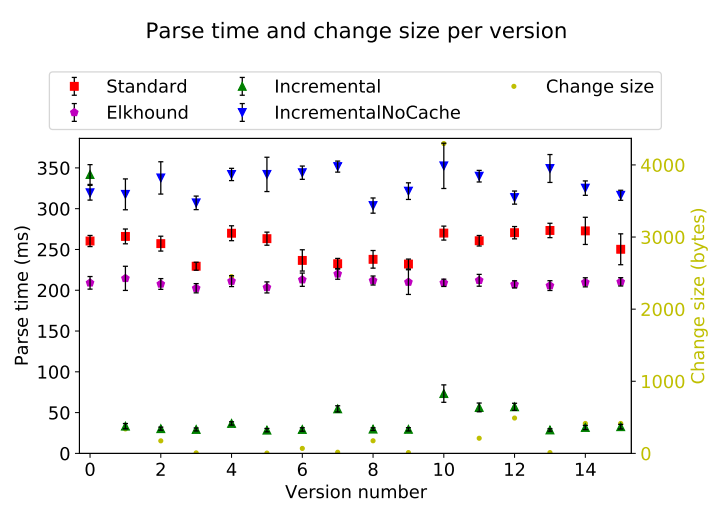

(d) Parse times of the nabl repository.

Figure 5.5: Parse times of the last 16 versions of several files and repositories from GitHub, including imploding and tokenization. The red squares represent the Standard JSGLR2 parser, the purple pentagons represent the Elkhound parser variant, the blue downward-pointing triangles represent the ISGLR parser in batch mode (i.e., without cache), the green upwardpointing triangles represent the ISGLR parser in incremental mode, the blue downwardpointing three-pointed stars represent the Tree-sitter parser in batch mode, and the green upward-pointing three-pointed stars represent the Tree-sitter parser in incremental mode. The yellow dots indicate the size of the changes in a particular version, which is the sum of the number of removed and inserted characters, on the second y-axis.

Table 5.6: Average parse times for the different languages in the evaluation corpus, as shown in Table 5.1, for three JSGLR2 parser variants and Tree-sitter. The values indicate the parse time in milliseconds, including imploding and tokenization.

\begin{tabular}{c|r|r|r|r|r|r} 
Language & Standard & Elkhound & $\begin{array}{r}\text { Incremental } \\
\text { (no cache) }\end{array}$ & Incremental & $\begin{array}{r}\text { Tree-sitter } \\
\text { (no cache) }\end{array}$ & Tree-sitter \\
\hline Average & \multicolumn{1}{|c|}{ (n10.266 } & 630.811 & 1007.738 & 90.302 & - & - \\
\hline Java & 2033.161 & 1579.048 & 2518.010 & 217.591 & 233.929 & 19.715 \\
WebDSL & 241.125 & 185.905 & 303.368 & 27.597 & - & - \\
SDF3 & 156.514 & 127.480 & 201.835 & 25.719 & - & -
\end{tabular}




\subsection{Memory Benchmark Results}

This section shows the performance results of the ISGLR parser in terms of memory usage, compared to other parser variants in JSGLR2. All benchmark results in this section show the average memory performance over 10 benchmark iterations, where the error bars in the plots indicate the minimum and maximum measurement for that data point. Note that the error bars are barely visible because the memory usage was almost constant in all cases. For these memory benchmarks, we measured the full JSGLR2 parsing pipeline, which includes imploding and tokenization, as shown in the bottom row of Figures 2.10 and 3.12. Note that we only measured the memory usage when running the parsers in batch mode.

Memory Allocations Figure 5.7 shows the memory usage in terms of memory allocations. The memory usage of all parser variants tends to increase linearly with respect to the size of the input files for almost all files of the corpus. The average memory usage is shown in Table 5.8. On average, the ISGLR parser uses $23 \%$ more memory allocations than the Standard JSGLR2 parser variant and $67 \%$ more than the Elkhound variant.

We observe that Figure 5.7(a) shows some outliers where the memory usage is lower than for other files. After manual inspection, these Java files appeared to be abstract classes with a large number of documentation comments. This results in a smaller AST compared to a file of similar size that contains less documentation, therefore reducing memory usage in the imploder and tokenizer.

Table 5.8: Average memory allocations for the different languages in the evaluation corpus, as shown in Table 5.1, for three JSGLR2 parser variants. The values indicate the number of bytes allocated in the memory per character in the input, averaged over 100 input files.

\begin{tabular}{c|r|r|r} 
Language & Standard & Elkhound & Incremental \\
\hline Average & 2480 & 1826 & 3042 \\
\hline Java & 2379 & 1713 & 2878 \\
WebDSL & 2287 & 1653 & 2782 \\
SDF3 & 2774 & 2113 & 3465
\end{tabular}

Cache Size Figure 5.9 shows the size of the parser cache after parsing, calculated as the difference between the memory footprints before and after parsing. We can see that the parsers that only support batch parsing indeed store no data that should be available during a subsequent parse. The incremental parser does store this data, the size of which seems to increase linearly with respect to the input file size, similar to the number of memory allocations. The average memory size of the cache is shown in Table 5.10.

Table 5.10: Average memory size of the cache for the different languages in the evaluation corpus, as shown in Table 5.1, for three JSGLR2 parser variants. The values indicate the number of bytes allocated in the memory per character in the input, averaged over 100 input files.

\begin{tabular}{c|r|r|r} 
Language & Standard & Elkhound & Incremental \\
\hline Average & 0 & 0 & 270 \\
\hline Java & 0 & 0 & 224 \\
WebDSL & 0 & 0 & 251 \\
SDF3 & 0 & 0 & 336
\end{tabular}




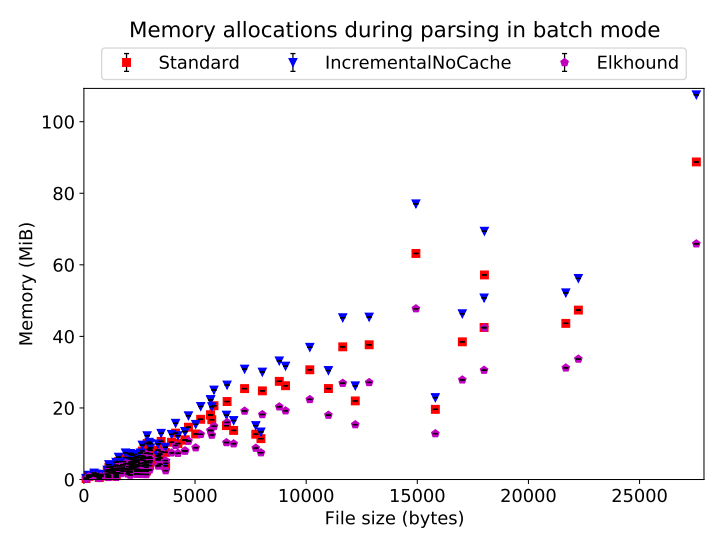

(a) When parsing Java.

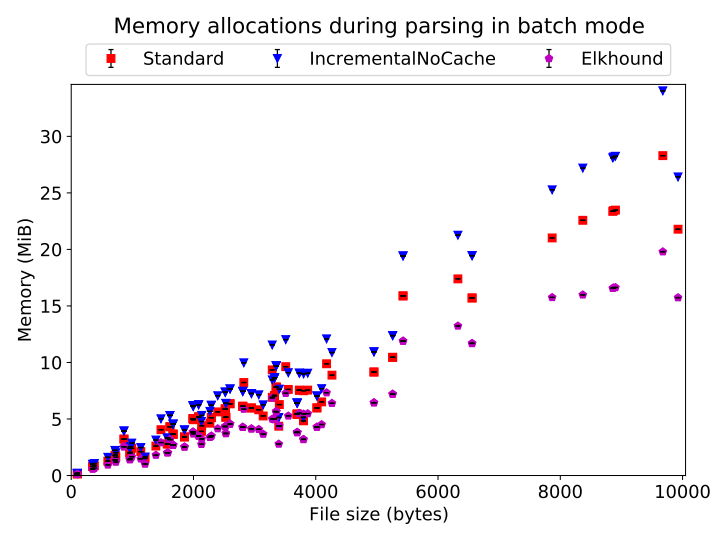

(b) When parsing WebDSL.

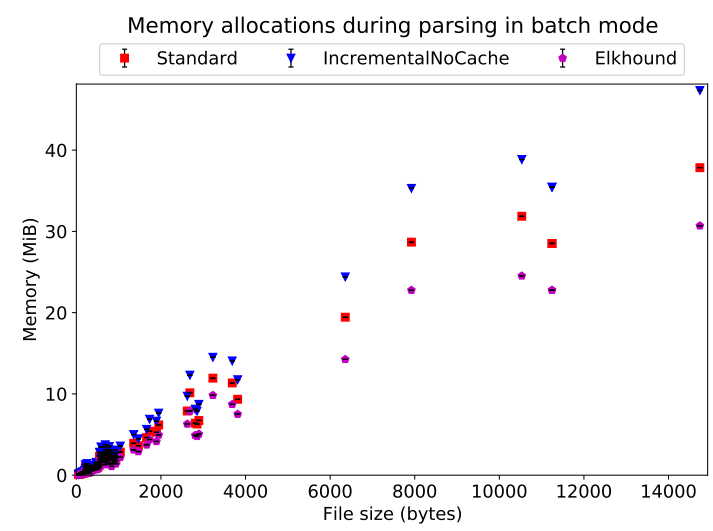

(c) When parsing SDF3.

Figure 5.7: Size of memory allocations during parsing for three variants of the JSGLR2 parser: the Standard parser (red squares), the Elkhound parser variant (purple pentagons), and the ISGLR parser in batch mode (blue triangles). Memory usage is calculated for 100 files of different sizes. 


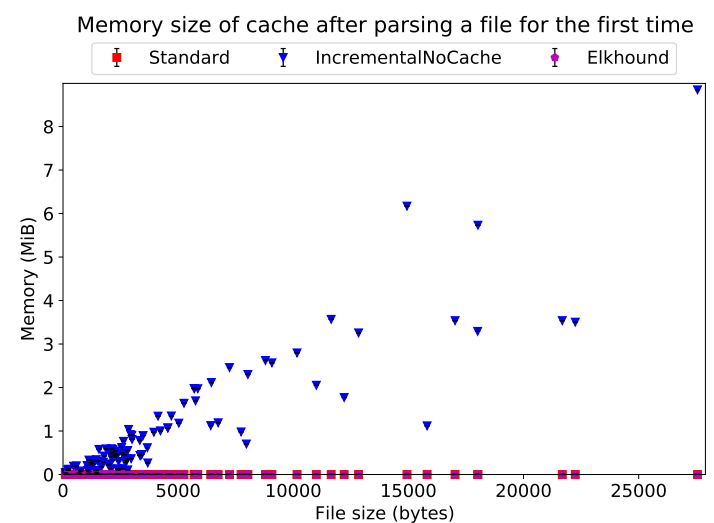

(a) When parsing Java.

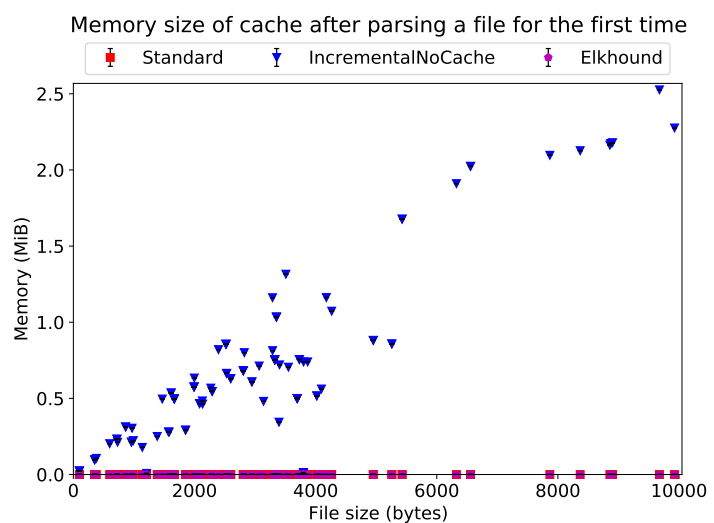

(b) When parsing WebDSL.

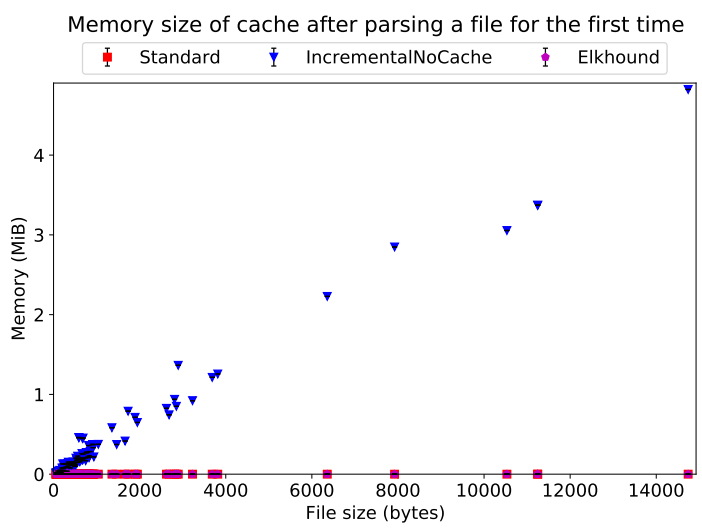

(c) When parsing SDF3.

Figure 5.9: Size of the cache after parsing a file for three variants of the JSGLR2 parser: the Standard parser (red squares), the Elkhound parser variant (purple pentagons), and the ISGLR parser in batch mode (blue triangles). Memory usage is calculated for 100 files of different sizes. 


\subsection{Threats to Validity}

The chosen method of performance evaluation has several concerns that might affect its validity. In this section, we discuss these threats to validity and to what extent we countered them.

Sampling Bias The selection of the evaluation corpus described in Section 5.1.1 raises the concern of sampling bias. It is not viable to evaluate the ISGLR parser with all possible languages and all available sources in that language, so selecting a subset of these as corpus is necessary, although it might not be representative. For the evaluation corpus, we selected languages that have different features and use cases to cover a range of languages that is as wide as possible.

Similarly, we need to select a part of the history of a project to use as input for the benchmark, as the full history of projects on GitHub consists of thousands of commits. Some commits may be smaller or larger than others, so by only taking the last 16 versions, we might introduce a bias towards a particular commit size. However, these commits typically still have larger change sizes than the size of a change would be when typing in the editor. The average change size of the sources in the chosen corpus is just below $1 \%$ of the input size, spread over multiple files, while typing in an editor leads to change sizes of only a couple of characters in a single file. Since we show that smaller change sizes typically take less time to parse incrementally, typing in the editor should not lead to performance issues. Conversely, a developer might switch branches in Git during development, which leads to larger change sizes than a single commit, since another branch may be multiple commits ahead or behind the current branch. However, we argue that the developer does not need an instant editor update in this scenario, implying that a slightly slower incremental update is acceptable in that case.

Finally, the parse time of the ISGLR parser does not only depend on the size of the change but also on what exactly changed. For example, changes to identifier names have less impact than changes that cause structural rearrangements in the AST. We have not attempted to quantify the "impactfulness" of a change, but we do suspect to have a sampling bias for this metric as well, besides the size of a change.

Correctness We do not prove the correctness of the ISGLR parser, so one might argue that the parser could return invalid results, possibly influencing the run time of the parser by taking certain shortcuts. Providing a full correctness proof of the ISGLR parser is outside the scope of this thesis, but we provide arguments for the necessity of certain parts of the ISGLR parsing algorithm in Chapter 3. However, we do have a suite of integration tests that is run automatically after pushing code to the main repository, ensuring that the basic functionality of the incremental parser is correct. In addition, the preprocessing step of the evaluation suite compares the resulting AST of an incremental parse with the AST of a batch parse for all files in the evaluation corpus. If the ASTs are not exactly equal, the entire benchmark is aborted. Of course, the concern of sampling bias also applies here: creating a test suite that covers all possible inputs is infeasible, and it remains possible that inputs outside the selected evaluation corpus might cause the ISGLR parser to return an incorrect result. 


\section{Chapter 6}

\section{Related Work}

Many other incremental parsing techniques have been published in the past years. In this chapter, we describe these approaches and compare them to our Incremental Scannerless Generalized LR (ISGLR) parsing algorithm. We make a distinction between LR parsers (Section 6.1), recursive descent parsers (Section 6.2), and parser combinators (Section 6.3).

\subsection{Incremental LR Parsers}

The incremental parsers described in this section are based on LR parsing, with a lexer that produces tokens, and a token-level grammar that the parser uses to generate a parse tree. Section 2.1 describes LR parsers in detail.

Merlin Bour, Refis, and Scherer (2018) present Merlin, a language server for OCaml. As a language server, it can answer queries from the user, like requesting the type of an identifier and navigating to the definition of an identifier.

To gain incrementality, Merlin stores checkpoints during parsing. These checkpoints are references to immutable parse stack objects, which only results in a small memory overhead since there is a lot of sharing within parse stacks. When the input to the parser is edited, Merlin restarts the parser from the last checkpoint belonging to the last unaffected token before the edit region. This approach has the disadvantage that an edit near the start of the input file would require almost a full reparse.

Harmonia Wagner and Graham (1998) present an Incremental LR (ILR) parsing algorithm as an improvement over several earlier approaches to incremental LR parsing. For a description of these earlier approaches and how their shortcomings are resolved, we refer to Wagner and Graham (1998, §2). Later, ${ }^{1}$ Wagner and Graham (1997b) extended this to allow nondeterministic parsing with the Incremental Generalized LR (IGLR) parsing algorithm, and Boshernitsan (2001) implemented an IGLR parser in the interactive setting of an IDE called Harmonia. Section 2.3 describes IGLR parsing in detail, as the ISGLR parsing algorithm presented in this work bases its incrementality on the IGLR parsing algorithm.

The ILR parser bases its incrementality on sentential-form parsing. It makes use of the assumption that the parse table reflects the grammar that it is generated from. The parser can reuse a parse node if the symbol that it represents is allowed to follow the parse state on top of the parse stack. The parse table generator provides information on which symbols are allowed to follow each parse state. This technique does not require state matching.

\footnotetext{
${ }^{1}$ These two papers appear to have been published in reverse order, but the paper of Wagner and Graham (1997b) on IGLR parsing refers back to the paper of Wagner and Graham (1998) on ILR parsing as "Tim A. Wagner and Susan L. Graham. Efficient and flexible incremental parsing, 1996. Submitted to ACM Trans. Program. Lang. Syst." In his PhD thesis, Wagner (1998, Chapters 6 \& 7) did present ILR parsing before IGLR parsing.
} 
However, this assumption of sentential-form parsing does not hold for production rules that have been disambiguated during parse table generation, e.g., using precedence and associativity rules. Wagner and Graham (1998) solve this issue by letting the ILR parser mark parse nodes as fragile when they represent such a production rule. If the parser encounters a fragile node during an incremental parse, it will always break it down. The IGLR parser does not need to track fragility, because it does use state matching, which (together with the lookahead test described in Section 2.3) also solves this issue.

\subsubsection{Parsers Based on Wagner's Approach}

The three incremental parsing approaches described in this section are all based on the ILR parsing algorithm by Wagner and Graham (1998). All of these approaches allow language composition, but in a less transparent way than scannerless parsing does. The first two approaches do not allow non-deterministic (IGLR) parsing, which is considered an advantage by their authors since it forces language designers to create non-ambiguous grammars, which prevents accidental ambiguities to cause errors in the editor. In contrast, scannerless parsing heavily relies on non-deterministic parsing, as described in Section 3.2.

Eco Diekmann and Tratt (2014) present Eco, a language composition editor. It acts as a normal text editor, but internally it uses structural (also known as syntax-directed) editing to make sure that the user always operates on a valid syntax tree. Adding custom grammars to Eco is possible, but this is "mainly used for testing" 2 and not documented.

The Eco editor has a fixed list of combinations of languages that it allows. A user can edit this list in configuration files. ${ }^{3}$ In the editor, a user can create fragments in a different language by creating language boxes, which are zero-width boundaries around text fragments that indicate which language a fragment is written in. Language boxes can be nested arbitrarily deep.

Tree-sitter Brunsfeld (2018) presents Tree-sitter, a parser generator that generates incremental parsers. It is integrated into widely-adopted editors like Atom and Neovim and supports many General-Purpose Languages (GPLs). Language designers can generate Treesitter parsers by writing a grammar in JavaScript and running the generator with that grammar as input. ${ }^{4}$

Language composition is possible in Tree-sitter by manually instructing the parser which ranges of the input should be parsed in which language. It is possible to automate this by adding explicit fences to the host language (e.g., "<?= . . ? ?" for the PHP language), then detecting parse nodes corresponding to those constructs, and use the range of such a parse node to call the parser for the embedded language. ${ }^{5}$

Lezer Haverbeke (2019) presents Lezer, a parser generator inspired by Tree-sitter that is built into CodeMirror, ${ }^{6}$ a code editor that can be run inside a web browser. Lezer supports GLR parsing in an opt-in fashion by allowing the grammar designer to annotate which production rules may allow the parser to fork. Lezer's lexer is integrated into the parser, allowing it to be contextual: token definitions are allowed to overlap, "as long as such tokens can't occur in the same place anywhere in the grammar". ${ }^{7}$ This also allows the layout definitions to depend on the context.

\footnotetext{
${ }^{2}$ https: //github.com/softdevteam/eco/pull/150

${ }^{3}$ https://github.com/softdevteam/eco/pull/238

${ }^{4}$ https://tree-sitter.github.io/tree-sitter/creating-parsers

${ }^{5}$ https://tree-sitter.github.io/tree-sitter/using-parsers\#multi-language-documents

${ }^{6}$ https://codemirror.net/

${ }^{7}$ https://lezer.codemirror.net/docs/guide/\#contextual-tokenization
} 
Lezer allows language composition directly in the grammar specification, as long as "the nested syntax has a clearly identifiable end token". ${ }^{8}$ However, unlike Tree-sitter, Lezer grammars allow directly importing a nested grammar and declaratively specifying the boundary tokens. This comes very close to the seamless language composition that scannerless parsing allows, and is sufficient for the grammars of GPLs that are currently published.

\subsection{Incremental Recursive Descent Parsers}

This section describes incremental parsing approaches based on recursive descent (or topdown) parsing. Unlike $\mathrm{LR}(k)$ parsing, which builds a parse tree from the bottom up, recursive descent parsers start parsing from the top down. Starting with the start symbol, they try to recognize the input by enumerating all production rules for that symbol and recursively do this search for all symbols in a production.

Incremental LL(1) Yang (1993) and Li (1995), among others, have introduced algorithms for incremental LL(1) parsing. Both incremental LL(1) parsing algorithms "cut" the previous parse tree at the location of an edit: say that the input $x y z$ is edited to $x \bar{y} z$, parse tree $X$ is equal to the previous parse tree with holes at the locations where the parse nodes for substrings $y$ and $z$ would be, and $Z$ is a list of subtrees that represent parts of $z$. Then, the parser initializes the LL(1) parsing algorithm as if it had already parsed $X$, with the sequences $\bar{y}$ and $Z$ as lookahead. To some extent, this is comparable with how ISGLR breaks down changed parse nodes and stores potentially reusable parse nodes on the input stack.

The algorithm of Yang (1993) decides whether it can reuse a subtree in $Z$ by using its first terminal node to index the parse table, whereas $\mathrm{Li}$ (1995) improved this by allowing the parse table to contain non-terminals so that the parser can use the symbol in the root of a subtree to determine the next parse action. The latter approach is equal to how the ILR parser by Wagner and Graham (1997b) tests if it can reuse a parse node.

Incremental Packrat Parsing Packrat parsing (Ford 2002) is an alternative approach to recursive descent parsing that memoizes intermediate results. The so-called memo table is a table that saves for every input position whether parsing a production rule at that position succeeds and how much of the input it spans. The memo table is sparse because it only stores the entries that the parser needs during parsing.

Dubroy and Warth (2017) add incrementality to packrat parsing by taking the memo table from a previous parse, removing entries from it that can conflict with the edit, and starting the parser with this updated memo table. They have implemented this parsing algorithm in Ohm, a parser generator toolkit based on Parsing Expression Grammars (Warth, Dubroy, and Garnock-Jones 2016). The source code for this parser and some visualizations can be found online. ${ }^{9}$

The algorithm that updates the memo table removes all entries that overlap with the positions touched by the edit, including entries that required to look forward to these positions to make a proper decision. For example, the parser can only recognize a number consisting of multiple digits if the position after the number is not a digit. Because recursive descent parsers use backtracking, the lookahead is theoretically unlimited, so in the worst case, the update algorithm needs to test for all entries of the memo table whether it should remove them. However, the performance of the incremental packrat parser in practice suggests that this is often not the case.

\footnotetext{
${ }^{8}$ https://lezer.codemirror.net/docs/guide/\#grammar-nesting

${ }^{9}$ https://ohmlang.github.io/sle17/
} 


\subsection{Incremental Parser Combinators}

Bernardy and Claessen (2015) present an incremental parsing algorithm that makes use of a divide-and-conquer algorithm using parser combinators. The algorithm is implemented in the Yi editor ${ }^{10}$ (Bernardy 2008). They also convert a grammar from Backus-Naur Form (BNF) to a variant of Chomsky Normal Form (CNF) where every production rule is transformed into a binary tree of productions to reduce the height of the parse tree.

The incremental parser of Bernardy and Claessen (2015) assumes lazy access to the parse tree. For example, when a user has an editor of a file open, they only view a small part of this file, and therefore only this part of the file needs to be parsed to perform syntax highlighting. When the user scrolls down in the file, the parser can resume from where it left off.

The parser of $\mathrm{Yi}$ is incremental because it caches intermediate parse results. Bernardy describes this in a blog post about Yi: "For given positions in the input (say every half-page), we will store a partially evaluated state of the parsing automaton. Whenever the input is modified, the new parsing result will be computed by using the most relevant cached state, and applying the new input to it. The cached states that became invalidated will also be recomputed on the basis of the most relevant state."11

\footnotetext{
${ }^{10}$ https://github.com/yi-editor/yi/

${ }^{11}$ https://yi-editor.github.io/posts/2014-๑9-๑4-incremental-parsing/
} 


\section{Chapter 7}

\section{Conclusion}

This thesis presents the Incremental Scannerless Generalized LR (ISGLR) parsing algorithm and answers the following research question:

What are the Effects of Combining Scannerless and Incremental GLR Parsing?

To answer this research question, we combined SGLR parsing and IGLR parsing into the ISGLR parsing algorithm and implemented it in the context of Spoofax. The differences of these two parsing algorithms with respect to GLR parsing are orthogonal to each other and are combined without difficulties. While the algorithmic differences are orthogonal, we show that there exist non-trivial interactions between these two techniques.

The fact that scannerless parsing (with character-level grammars) relies on non-deterministic parsing for disambiguation has a negative impact on incrementality. A parse node is irreusable when the parser creates it while exploring multiple possibilities to achieve unbounded lookahead, which is the case for $27 \%$ of all parse nodes on average in the evaluated corpus. The ISGLR parser creates many of these irreusable parse nodes when parsing the layout between symbols or when parsing literal keywords that overlap with identifiers. If a production rule ends with a fencing character (like curly brackets $(\{\})$ or semicolons $(;))$, the parse node that they terminate can be reused in a subsequent incremental parse. In general, an incremental parser can reuse more of a previous parse tree if the grammar allows it to parse the input more deterministically.

Nonetheless, we show that the ISGLR parsing algorithm performs better than the batch SGLR parsing algorithm in typical scenarios. The majority of the irreusable parse nodes does not get exposed on top of the input stack during a subsequent incremental parse, so on average, the ISGLR parser can reuse $99 \%$ of a previous parse tree. When parsing from scratch, the ISGLR parser has a $24 \%$ run time overhead compared to the SGLR parser, but when parsing incrementally for changes that are smaller than $1 \%$ of the input size on average, it has a $9 \times$ speedup.

\subsection{Future Work}

Balanced Lists Wagner and Graham (1998) represent list production rules as balanced binary trees in the parse tree, while SDF3 normalizes list productions as left-recursive constructs. Using balanced trees reduces the height of the parse tree to $\mathcal{O}(\log n)$ (where $n$ is the number of characters) instead of $\mathcal{O}(n)$ in the worst case. Reducing the height of the parse tree reduces the length of the spine that needs to be broken down during an incremental parse.

Index Parse Table With Productions Wagner and Graham (1998) adapt the parse table generator so that it allows their Incremental LR (ILR) parser to retrieve the applicable actions 
from the action table using a state-non-terminal pair. This information is already available during parse table generation, but SDF3 does not store this in the parse table. The ISGLR parsing algorithm currently calculates the applicable actions for a non-terminal based on the first character in its parse node. Only when a parse node can be reused, it accesses the goto table using the production rule of the parse node. For this thesis, it was out of scope to improve the parse table generator, but it would be interesting to see if this improves the performance of the ISGLR parser.

Layout as Skip Productions The lexer of Lezer (Haverbeke 2019) is integrated into its parser, and this allows layout definitions to depend on the context, among other things. Their parser essentially "skips" any layout that matches these definitions. Reusing some ideas from Lezer might reduce non-determinism during parsing that is related to layout.

Incremental Error Recovery De Jonge et al. (2012) implement error recovery for SGLR parsing, and Wagner (1998, Chapter 8) and Brunsfeld (2018) implement error recovery for IGLR parsing and ILR parsing, respectively. Error recovery is currently a work in progress for JSGLR2 (Denkers 2018), and it would be interesting to combine this implementation with the incremental parser.

Integrate Into Spoofax While Spoofax already supports incremental type checking (Aerts 2019) and incremental compilation (Smits, Konat, and Visser 2020), these implementations are coarse-grained: a changed file will need to be fully reprocessed to some degree. They would benefit from detecting which elements in the AST have changed in a file based on the result from the incremental parser.

Incremental Editor Update Some tasks in the editor plugin that is generated by Spoofax would also benefit from incremental updates from the parser. The editor plugin performs syntax highlighting based on the tokens produced by the tokenization post-processing task. In addition, it generates a program outline based on the AST. Both of these tasks can be made incremental using the result from the incremental parser. Conversely, an editor might already have a built-in way of detecting textual updates, which can be used to improve the diff part of the ISGLR parsing algorithm. 


\section{Bibliography}

Aerts, Taico (2019). 'Incrementalizing Statix: A Modular and Incremental Approach for Type Checking and Name Binding using Scope Graphs'. Master's thesis. Delft University of Technology. URL: https : / / resolver . tudelft . nl / uuid : 3e@ea516-3058-4b8c-bfb65 e846c4bd982.

van Antwerpen, Hendrik, Casper Bach Poulsen, Arjen Rouvoet, and Eelco Visser (2018). 'Scopes as types'. In: Proceedings of the ACM on Programming Languages 2.OOPSLA. Dor: $10.1145 / 3276484$.

Bernardy, Jean-Philippe (2008). 'Yi: an editor in haskell for haskell'. In: Proceedings of the 1st ACM SIGPLAN Symposium on Haskell, Haskell 2008, Victoria, BC, Canada, 25 September 2008. Edited by Andy Gill. ACM, pages 61-62. IsBN: 978-1-60558-064-7. Dor: 10 . 1145/1411286 . 1411294.

Bernardy, Jean-Philippe and Koen Claessen (2015). 'Efficient parallel and incremental parsing of practical context-free languages'. In: Journal of Functional Programming 25. Dor: 10 . $1017 /$ S0956796815000131.

Boshernitsan, Marat (2001). HARMONIA: A Flexible Framework for Constructing Interactive Language-Based Programming Tools. Technical report. URL: https : / / www2 . eecs . berkeley . edu/Pubs/TechRpts/2001/CSD-01-1149.pdf.

Bour, Frédéric, Thomas Refis, and Gabriel Scherer (2018). 'Merlin: a language server for OCaml (experience report)'. In: Proceedings of the ACM on Programming Languages 2.ICFP. DOI: $10.1145 / 3236798$.

Bravenboer, Martin, Karl Trygve Kalleberg, Rob Vermaas, and Eelco Visser (2008). 'Stratego/XT 0.17. A language and toolset for program transformation'. In: Science of Computer Programming 72.1-2, pages 52-70. DoI: 10.1016/j . scico.2007.11.003.

Brunsfeld, Max (GitHub) (2018-03-04). Tree-sitter. A New Parsing System for Programming Tools. Talk at: Free and Open source Software Developers' European Meeting, FOSDEM 2018, Brussels, Belgium, February 3-4, 2018. uRL: https: //archive. fosdem.org/2018/schedule/event/code_ tree_sitter/.

Denkers, Jasper (2018). 'A Modular SGLR Parsing Architecture for Systematic Performance Optimization'. Master's thesis. Delft University of Technology. URL: https : / / resolver . tudelft.nl/uuid:7d9f9bcc-117c-4617-860a-4e3e-0bbc8988.

Diekmann, Lukas and Laurence Tratt (2014). 'Eco: A Language Composition Editor'. In: Software Language Engineering - 7th International Conference, SLE 2014, Västeras, Sweden, September 15-16, 2014. Proceedings. Edited by Benoît Combemale, David J. Pearce, Olivier Barais, and Jurgen J. Vinju. Volume 8706. Lecture Notes in Computer Science. Springer, pages 82101. ISBN: 978-3-319-11244-2. DOI: 10.1007/978-3-319-11245-9_5.

Dubroy, Patrick and Alessandro Warth (2017). 'Incremental packrat parsing'. In: Proceedings of the 10th ACM SIGPLAN International Conference on Software Language Engineering, SLE 2017, Vancouver, BC, Canada, October 23-24, 2017. Edited by Benoît Combemale, Marjan 
Mernik, and Bernhard Rumpe. ACM, pages 14-25. ISBN: 978-1-4503-5525-4. Dor: 10.1145/ 3136014.3136022.

Ford, Bryan (2002). 'Packrat parsing: simple, powerful, lazy, linear time, functional pearl'. In: Proceedings of the seventh ACM SIGPLAN international conference on Functional Programming (ICFP 2002), pages 36-47. DOI: 10.1145/581478.581483.

Fourment, Mathieu and Michael R. Gillings (2008). 'A comparison of common programming languages used in bioinformatics'. In: BMC Bioinformatics 9. Dor: 10.1186/1471-2105-9-82.

Haverbeke, Marijn (2019-09-03). Lezer. URL: https : / / marijnhaverbeke. nl /blog/ lezer . html (visited on 2021-07-07).

Jalili, Fahimeh and Jean H. Gallier (1982). ‘Building Friendly Parsers'. In: POPL, pages 196206.

Johnson, S. C. (1975). YACC: Yet Another Compiler-Compiler. Technical report CS-32. Murray Hill, NJ, USA: AT\&T Bell Laboratories.

Johnson, Walter L., James H. Porter, Stephanie I. Ackley, and Douglas T. Ross (1968). 'Automatic generation of efficient lexical processors using finite state techniques'. In: Communications of the ACM 11.12, pages 805-813. DoI: 10.1145/364175.364185.

de Jonge, Maartje, Lennart C. L. Kats, Eelco Visser, and Emma Söderberg (2012). 'Natural and Flexible Error Recovery for Generated Modular Language Environments'. In: ACM Transactions on Programming Languages and Systems 34.4, page 15. Dor: 10.1145 / 2400676. 2400678.

Kats, Lennart C. L. and Eelco Visser (2010). 'The Spoofax language workbench: rules for declarative specification of languages and IDEs'. In: Proceedings of the 25th Annual ACM SIGPLAN Conference on Object-Oriented Programming, Systems, Languages, and Applications, OOPSLA 2010. Edited by William R. Cook, Siobhán Clarke, and Martin C. Rinard. Reno/Tahoe, Nevada: ACM, pages 444-463. ISBN: 978-1-4503-0203-6. Dor: 10 . 1145 / 1869459 . 1869497.

Knuth, Donald E. (1965). 'On the translation of languages from left to right'. In: Information and control 8.6.

Lesk, M. E. and E. Schmidt (1975). Lex - A Lexical Analyzer Generator. Technical report CS-39. Murray Hill, NJ, USA: AT\&T Bell Laboratories.

Li, Warren X. (1995). 'A Simple and Efficient Incremental LL(1) parsing'. In: SOFSEM 95, 22nd Seminar on Current Trends in Theory and Practice of Informatics, Milovy, Czech Republic, November 23 - December 1, 1995, Proceedings. Edited by Miroslav Bartosek, Jan Staudek, and Jirí Wiedermann. Volume 1012. Lecture Notes in Computer Science. Springer, pages 399404. ISBN: 3-540-60609-2.

McPeak, Scott and George C. Necula (2004). 'Elkhound: A Fast, Practical GLR Parser Generator'. In: Compiler Construction, 13th International Conference, CC 2004, Held as Part of the Joint European Conferences on Theory and Practice of Software, ETAPS 2004, Barcelona, Spain, March 29 - April 2, 2004, Proceedings. Volume 2985. Lecture Notes in Computer Science. Springer, pages 73-88. ISBN: 3-540-21297-3. DOI: 10.1007/978-3-540-24723-4_6.

MetaBorg (2016). The Spoofax Language Workbench. URL: https : / / www . metaborg . org / en / latest/ (visited on 2021-07-07).

Mickunas, M. Dennis, Ronald L. Lancaster, and Victor B. Schneider (1976). 'Transforming LR(k) Grammars to LR(1), SLR(1), and $(1,1)$ Bounded Right-Context Grammars'. In: Journal of the ACM 23.3, pages 511-533. Dor: 10.1145/321958.321972.

Rekers, Jan (1992-01). 'Parser Generation for Interactive Environments'. PhD thesis. Amsterdam, The Netherlands: University of Amsterdam. URL: https : / / homepages . cwi . nl / $\sim$ paulk/dissertations/Rekers.pdf.

Salomon, D. J. and G. V. Cormack (1989). 'Scannerless NSLR(1) parsing of programming languages'. In: SIGPLAN Not. 24.7. Dor: 10.1145/74818.74833.

Sipser, Michael (2012). Introduction to the Theory of Computation. 3rd. Cengage Learning. ISBN: 978-1-133-18779-0. 
Smits, Jeff, Gabriël Konat, and Eelco Visser (2020). ‘Constructing Hybrid Incremental Compilers for Cross-Module Extensibility with an Internal Build System'. In: Programming Journal 4.3, page 16. DOI: 10.22152/programming-journal .org/2020/4/16.

de Souza Amorim, Luis Eduardo, Michael J. Steindorfer, Sebastian Erdweg, and Eelco Visser (2018). 'Declarative specification of indentation rules: a tooling perspective on parsing and pretty-printing layout-sensitive languages'. In: Proceedings of the 11th ACM SIGPLAN International Conference on Software Language Engineering, SLE 2018, Boston, MA, USA, November 05-06, 2018. Edited by David Pearce 0005, Tanja Mayerhofer, and Friedrich Steimann. ACM, pages 3-15. ISBN: 978-1-4503-6029-6. DOI: 10.1145/3276604.3276607.

de Souza Amorim, Luis Eduardo, Michael J. Steindorfer, and Eelco Visser (2018). 'Towards Zero-Overhead Disambiguation of Deep Priority Conflicts'. In: Programming Journal 2.3, page 13. DOI: 10.22152/programming-journal .org/2018/2/13.

de Souza Amorim, Luis Eduardo and Eelco Visser (2020). 'Multi-purpose Syntax Definition with SDF3'. In: Software Engineering and Formal Methods - 18th International Conference, SEFM 2020, Amsterdam, The Netherlands, September 14-18, 2020, Proceedings. Edited by Frank S. de Boer and Antonio Cerone. Volume 12310. Lecture Notes in Computer Science. Springer, pages 1-23. IsBN: 978-3-030-58768-0. DOI: 10.1007/978-3-030-58768-๑_1.

Tomita, Masaru (1985). 'An Efficient Context-Free Parsing Algorithm for Natural Languages'. In: IJCAI, pages 756-764.

Visser, Eelco (1997-07). Scannerless Generalized-LR Parsing. Technical report P9707. Programming Research Group, University of Amsterdam.

Vollebregt, Tobi, Lennart C. L. Kats, and Eelco Visser (2012). 'Declarative specification of template-based textual editors'. In: International Workshop on Language Descriptions, Tools, and Applications, LDTA '12, Tallinn, Estonia, March 31 - April 1, 2012. Edited by Anthony Sloane and Suzana Andova. ACM, pages 1-7. ISBN: 978-1-4503-1536-4. DoI: $10.1145 / 2427048.2427056$.

Wagner, Tim A. (1998-03). 'Practical Algorithms for Incremental Software Development Environments'. PhD thesis. EECS Department, University of California, Berkeley. URL: https : //www2. eecs. berkeley. edu/Pubs/TechRpts/1998/5885.html.

Wagner, Tim A. and Susan L. Graham (1997a). 'General Incremental Lexical Analysis'.

Wagner, Tim A. and Susan L. Graham (1997b). 'Incremental Analysis of real Programming Languages'. In: PLDI, pages 31-43.

Wagner, Tim A. and Susan L. Graham (1998). 'Efficient and Flexible Incremental Parsing'. In: ACM Transactions on Programming Languages and Systems 20.5, pages 980-1013. DoI: $10.1145 / 293677.293678$.

Warth, Alessandro, Patrick Dubroy, and Tony Garnock-Jones (2016). 'Modular semantic actions'. In: Proceedings of the 12th Symposium on Dynamic Languages, DLS 2016, Amsterdam, The Netherlands, November 1, 2016. Edited by Roberto Ierusalimschy. ACM, pages 108-119. ISBN: 978-1-4503-4445-6. DOI: 10.1145/2989225.2989231.

Willink, Edward D. (2001). 'Meta-compilation for C++'. British Library, EThOS. PhD thesis. University of Surrey, Guildford, UK. URL: http: / / ethos.bl .uk/OrderDetails . do? uin=uk . bl. ethos. 370061.

Yang, Wuu (1993). 'An Incremental LL(1) Parsing Algorithm'. In: Inf. Process. Lett. 48.2, pages $67-72$. 
This page is intentionally left blank. 


\section{Acronyms}

AST Abstract Syntax Tree

BNF Backus-Naur Form

CNF Chomsky Normal Form

CST Concrete Syntax Tree

CSV Comma-Separated Values

DSL Domain-Specific Language

EOF End-of-File

GLR Generalized LR

GPL General-Purpose Language

GSS Graph-Structured Stack

IDE Integrated Development Environment

IGLR Incremental Generalized LR

ILR Incremental LR

ISGLR Incremental Scannerless Generalized LR

JMH Java Microbenchmark Harness

JVM Java Virtual Machine

LR Left-to-right Rightmost-derivation

NSLR Noncanonical Simple LR

SDF3 Syntax Definition Formalism 3 (Vollebregt, Kats, and Visser 2012;

de Souza Amorim and Visser 2020)

SGLR Scannerless Generalized LR

SLR Simple LR 
This page is intentionally left blank. 


\section{Glossary}

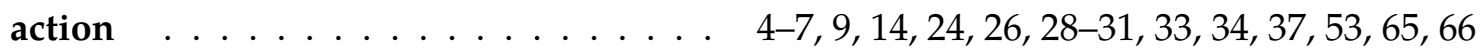

The three different types of actions listed in the action table that decide what the parser should do: Shift, Reduce, or Accept. Section 2.1.3 shows a full description of these actions.

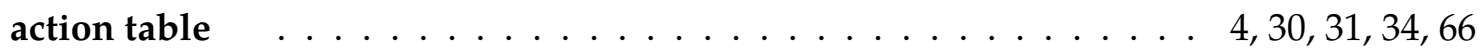

The part of the parse table that maps state-terminal pairs to actions.

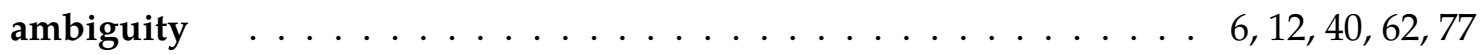

A part of the input stream that can be parsed in multiple ways, represented as a parse node with multiple derivations.

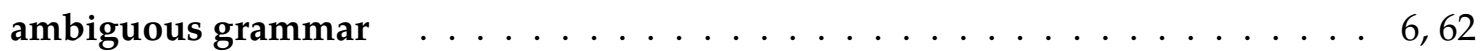
A context-free grammar for which there exists some string that can be parsed in multiple ways.

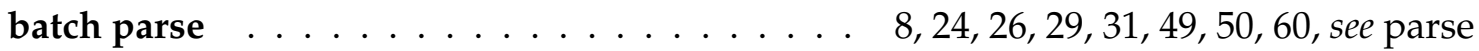
A single invocation of a parser without reusing previous results, i.e., not being incremental.

character class

A compact notation to describe a set of characters. For example, [A-Za-z] denotes the set of all letters in the English alphabet.

character node . . . . . . . . . . 10 13, 22, 26, 31-34, 36, 50, 53, 77

A leaf node of a parse tree for a scannerless parser, corresponding to a single character of the input string.

character-level grammar . . . . . . . . . . . . . . . . . 10, 12, 24, 65

A context-free grammar that is described using characters as terminal symbols.

conflict . . . . . . . . . . . . . . . . . . . . . 4, 6, 29-31

An entry in the action table that has more than one action.

context-free grammar . . . . 3-6, 10-12, 17-20, 24-31, 39, 43, 50, 51, 53, 55, 61-65, 115

A description of a language, as defined in Section 2.1.

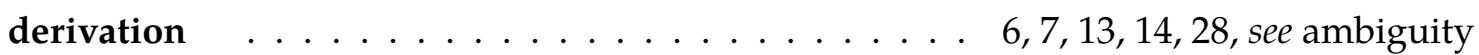
A possible way of parsing the input represented by the parse node that contains this derivation.

diff

$17,21,22,31,33,53,55,66$

A list of changes between two strings of characters. 


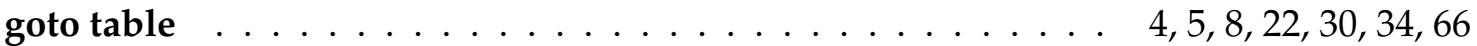
The part of the parse table that maps state-non-terminal pairs to other parse states.

grammar normalization . . . . . . . . . . . 10 12-12, 17, 19, 28-30, 65 The process of transforming a high-level SDF3 grammar specification to a specification that only uses core constructs of SDF3.

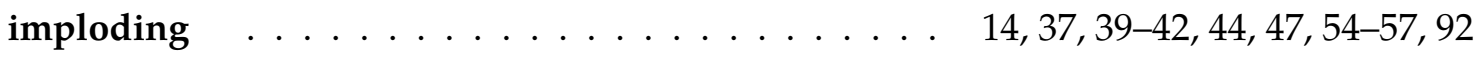
The process of reducing a Concrete Syntax Tree (CST) to an Abstract Syntax Tree (AST).

incremental parse . . . 8, 9, 17, 22-24, 26, 29-31, 36, 49, 50, 52, 53, 60, 62, 65, see parse An invocation of the parser on an input that received a change with respect to a previous version. During an incremental parse, the parser tries to reuse subresults of a previous parse.

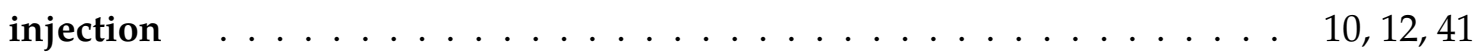
A production rule in a grammar that has only one symbol on its right-hand side.

input stack . . . . . . . . . . . 21, 22, 24, 26, 29-34, 36, 37, 49, 53, 63, 65 Used as input by the ISGLR parser. Contains both character nodes and parse nodes. If the parse node on top of the input stack may not be reused, it is broken down and its children are pushed back to the stack such that the first child ends up on top.

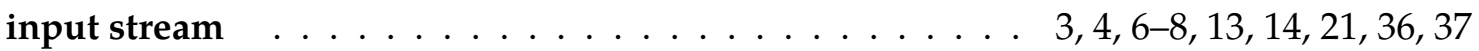

The input to a parser, usually a stream of characters. In non-scannerless parsers, a lexer transforms this input stream into a stream of tokens before parsing.

irreusable parse node $\quad \ldots . . .99,17,24,26,29,31,34,36,37,49,50,52,53,65,77$ A parse node that can never be reused during a subsequent incremental parse if it ends up at the top of the input stack, because it was created during non-deterministic parsing.

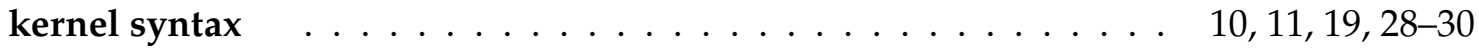

A low-level representation of production rules in SDF3, in which the sorts symbols (representing the non-terminals) are annotated with -CF or -LEX to remember whether it originates from context-free syntax or lexical syntax, respectively.

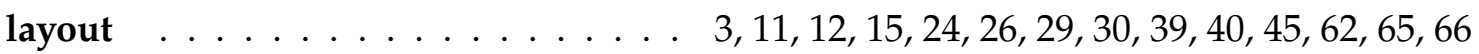
Parts of source code that do not contribute to the meaning of it, but only contribute to readability. Examples are comments (usually denoted with "//" or " $/ * * / ")$ and characters representing whitespace, such as space ( ' ', $U+0020)$, tab (' $\backslash t ', U+0009)$, and newline ( ' $\backslash n$ ', U+@०९A).

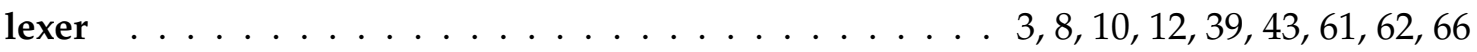
Used for parsers that recognize token-level grammars. Transforms an input stream of text characters into a stream of tokens that can be used as terminals.

lexical element . . . . . . . . . . . . . . . . . . . . . . . . 10-12,39 Elements in the grammar of a language that would correspond to a single token in tokenlevel grammars. Examples include identifier names, numbers, strings, and literals.

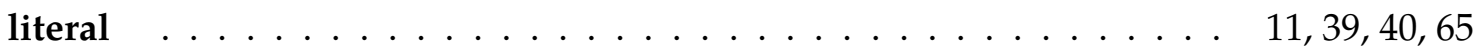

A non-varying lexical element in a context-free grammar. Examples include keywords and operators.

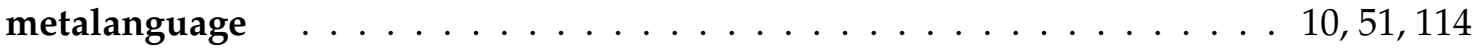

A language specifically used to describe another language, like SDF3. 
non-deterministic parsing $\ldots \ldots \ldots 6,9 \ldots \ldots, 17,24,29,32,52,53,61,62,65$ When a parser needs to fork the parse stack during parsing, exploring multiple parse states at once to achieve unbounded lookahead. This does not imply that the final result contains ambiguities.

non-terminal

$3,4,8,10,12,63,66$

Shorthand for non-terminal symbol. Non-terminal symbols can be used on both sides of production rules.

parse $\ldots \ldots \ldots . \ldots 8,13,23,57,63,77$, see batch parse \& incremental parse (noun) An invocation of the parser.

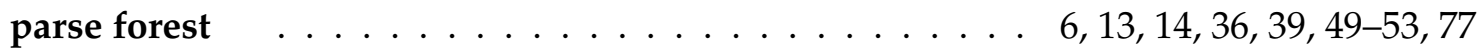
A parse tree that contains (or may contain) ambiguities. Multiple non-ambiguous parse trees could be constructed from a parse forest.

parse node . . . . . 4 4, 6-9, 13, 14, 17, 21-37, 39-44, 49, 50, 52, 53, 61-63, 65, 66, 77 A non-leaf node in a parse tree, corresponding to a production rule.

parse stack . . . . . . . . . . . . . . . . . 4-9, 13, 14, 22, 24-36, 61 Stores references to parse states, with the start state at the bottom and the current state at the top of the stack. Each link between two stack nodes stores a parse node or terminal node.

parse state $\ldots \ldots \ldots \ldots \ldots$. . . . . . . . . . 13, 22, 29, 30, 32-37, 53, 61, 66 A state of an LR-based parser automaton, used in the parse table and the parse stack.

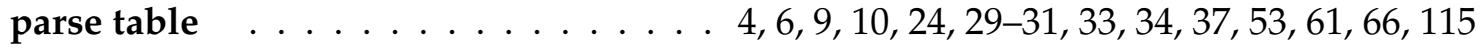
A two-dimensional table that represents the possible state transitions of an LR-based parser automaton. Consists of an action table and a goto table.

parse table generator . . . . . . . . . 4, 6, 10, 14, 24, 30, 31, 61, 62, 65, 66, 115 An algorithm that generates a parse table from the production rules of a context-free grammar.

parse tree $\ldots \ldots .3,4,6,8-10,17,20-24,26,29,31,32,39,40,43,44,49,61,63,65$ A tree-shaped representation of an input string, parsed according to a context-free grammar, containing parse nodes and terminal nodes.

production rule $\ldots . .33,4,8,10-14,26,28-31,34,35,39-41,44,45,52,53,62-66$ A rule in a context-free grammar of the form $A \rightarrow \alpha$ which maps non-terminal $A$ to a string of symbols $\alpha$.

sort $10-12,22,28-30,53$

(noun) A non-terminal symbol in SDF3.

spine

The path between a given node in a tree and the root of that tree.

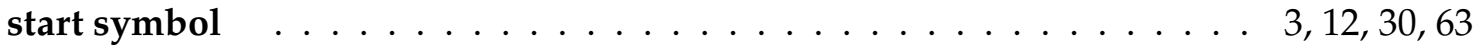

The root symbol of a context-free grammar.

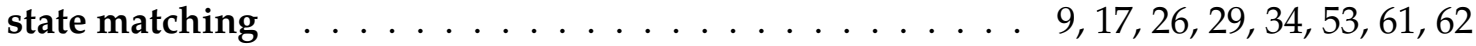
A test that determines whether unchanged parse nodes can be reused, which is the case when the parse state reference stored in the parse node is the same as the current parse state.

symbol . . . . . . . . . . . . . 3, 10-12, 14, 22, 29, 30, 33, 61, 63, 65

A symbol in a context-free grammar. Can be either a non-terminal symbol or a terminal symbol. 


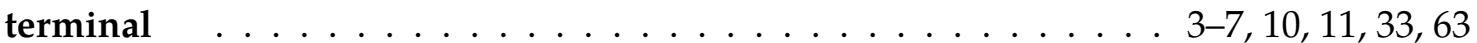

Shorthand for terminal symbol. Terminal symbols can only be used on the right-hand side of production rules.

token $\ldots \ldots \ldots \ldots$ 3, 4, 8-10, 12, 14, 21, 24, 39, 43-47, 51, 61-63, 66, 114 A substring of the input to the parser, used as terminal symbol when parsing tokenlevel grammars (Section 2.1) or generated after parsing with character-level grammars (Section 4.2).

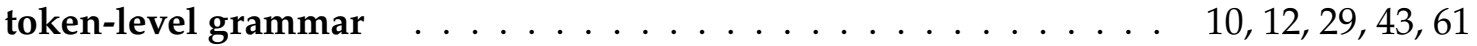
A context-free grammar that is described using tokens as terminal symbols.

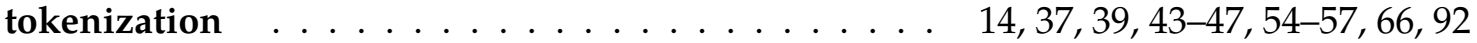
The process of generating a list of tokens from a parse tree and attaching these tokens to the corresponding Abstract Syntax Tree (AST).

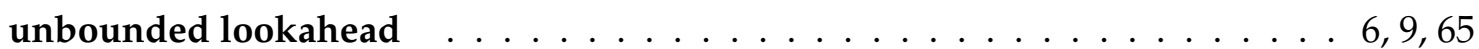

A property of a parser that allows it to look ahead an arbitrary number of terminal symbols during parsing. In Generalized LR (GLR) parsing, this is accomplished by non-deterministically parsing the input in pseudo-parallel, using multiple active parse stacks at once. 


\section{Appendix A}

\section{Full Evaluation Results}

This appendix contains the full evaluation results for the Incremental Scannerless Generalized LR (ISGLR) parser. These results are also available online at the Spoofax evaluation website for JSGLR2: https://www.spoofax.dev/jsglr2evaluation-site/2021-06-15_18:13/.

\section{A.1 Measurements}

The tables on the left are counts of parse nodes and character nodes after a parse for one version of the input has finished. For parse nodes, it also shows the number of ambiguous parse nodes and the number of irreusable parse nodes (percentages are relative to the "Parse Nodes Count" column).

The tables on the right are counts of certain events happening during parsing. It shows how many parse nodes were created during parsing and how many parse nodes were reused or rebuilt (percentages are relative to the "Parse Nodes Count" of the row at the same height in the left part of the table, i.e., the previous version of the parse forest). The "Shift" column shows how many parse nodes and character nodes the parser has shifted during the parse. The "Breakdown Count" column shows how many parse nodes were broken down during parsing (percentages are relative to the "Parse Nodes Count" column of the row at the same height in the left part of the table). The other "Breakdown" columns give a breakdown (pun intended) of the different types of breakdowns (percentages are relative to the "Breakdown Count" column), for which the full explanation can be found in Section 5.2.2.

A copy of Table 5.2 in Section 5.2.

\begin{tabular}{c|c|c|c|c|c|c|c|c}
\multirow{2}{*}{ Language } & \multicolumn{4}{|c|}{ Parse nodes $\%$ of total nodes) } & \multicolumn{3}{c}{ Breakdowns $\%$ of total breakdowns) } \\
& $\begin{array}{c}\text { Irre- } \\
\text { usable }\end{array}$ & Reused & $\begin{array}{c}\text { Broken } \\
\text { down }\end{array}$ & Rebuilt & $\begin{array}{c}\text { Contains } \\
\text { Change }\end{array}$ & $\begin{array}{c}\text { Irre- } \\
\text { usable }\end{array}$ & $\begin{array}{c}\text { No } \\
\text { actions }\end{array}$ & $\begin{array}{c}\text { Wrong } \\
\text { state }\end{array}$ \\
\hline Average & $27.25 \%$ & $99.12 \%$ & $0.67 \%$ & $0.48 \%$ & $40.68 \%$ & $56.23 \%$ & $0.00 \%$ & $3.08 \%$ \\
\hline Java & $19.08 \%$ & $99.60 \%$ & $0.17 \%$ & $0.08 \%$ & $51.35 \%$ & $46.67 \%$ & $0.00 \%$ & $1.98 \%$ \\
WebDSL & $21.32 \%$ & $99.55 \%$ & $0.28 \%$ & $0.15 \%$ & $51.35 \%$ & $43.76 \%$ & $0.00 \%$ & $4.89 \%$ \\
SDF3 & $41.36 \%$ & $98.21 \%$ & $1.57 \%$ & $1.21 \%$ & $19.35 \%$ & $78.28 \%$ & $0.00 \%$ & $2.38 \%$
\end{tabular}


Incremental parsing measurements for all languages.

\begin{tabular}{|c|c|c|c|c|c|c|c|c|c|c|c|c|c|c|c|}
\hline \multirow[b]{2}{*}{ Language } & \multicolumn{3}{|c|}{ Parse Nodes } & \multirow{2}{*}{$\begin{array}{c}\text { Character } \\
\text { Nodes } \\
\text { Count }\end{array}$} & \multirow[b]{2}{*}{ Language } & \multicolumn{3}{|c|}{ Parse Nodes } & \multicolumn{2}{|c|}{ Shift } & \multicolumn{5}{|c|}{ Breakdown } \\
\hline & Count & $\begin{array}{l}\text { Ambi- } \\
\text { guous }\end{array}$ & $\begin{array}{c}\text { Irre- } \\
\text { usable }\end{array}$ & & & Created & Reused & Rebuilt & $\begin{array}{l}\text { Parse } \\
\text { Node }\end{array}$ & $\begin{array}{c}\text { Character } \\
\text { Node }\end{array}$ & Count & $\begin{array}{l}\text { Contains } \\
\text { Change }\end{array}$ & $\begin{array}{c}\text { Irre- } \\
\text { usable }\end{array}$ & $\begin{array}{c}\text { No } \\
\text { Actions }\end{array}$ & $\begin{array}{l}\text { Wrong } \\
\text { State }\end{array}$ \\
\hline Average & 587518 & $0.34 \%$ & $27.25 \%$ & 336819 & Average & 7816 & $99.12 \%$ & $0.48 \%$ & 411 & 1072 & $0.67 \%$ & $40.68 \%$ & $56.23 \%$ & $0.00 \%$ & $3.08 \%$ \\
\hline Java & 1453416 & $0.00 \%$ & $19.08 \%$ & 852068 & Java & 13989 & $99.60 \%$ & $0.08 \%$ & 631 & 1849 & $0.17 \%$ & $51.35 \%$ & $46.67 \%$ & $0.00 \%$ & $1.98 \%$ \\
\hline WebDSL & 198475 & $0.00 \%$ & $21.32 \%$ & 101295 & WebDSL & 3733 & $99.55 \%$ & $0.15 \%$ & 324 & 589 & $0.28 \%$ & $51.35 \%$ & $43.76 \%$ & $0.00 \%$ & $4.89 \%$ \\
\hline SDF3 & 110664 & $1.01 \%$ & $41.36 \%$ & 57095 & SDF3 & 5725 & $98.21 \%$ & $1.21 \%$ & 279 & 778 & $1.57 \%$ & $19.35 \%$ & $78.28 \%$ & $0.00 \%$ & $2.38 \%$ \\
\hline
\end{tabular}

\section{A.1.1 Java}

Incremental parsing measurements for the Java language.

\begin{tabular}{|c|c|c|c|c|c|c|c|c|c|c|c|c|c|c|c|}
\hline \multirow[b]{2}{*}{ Source } & \multicolumn{3}{|c|}{ Parse Nodes } & \multirow{2}{*}{$\begin{array}{c}\text { Character } \\
\text { Nodes } \\
\text { Count }\end{array}$} & \multirow[b]{2}{*}{ Source } & \multicolumn{3}{|c|}{ Parse Nodes } & \multicolumn{2}{|c|}{ Shift } & \multicolumn{5}{|c|}{ Breakdown } \\
\hline & Count & $\begin{array}{l}\text { Ambi- } \\
\text { guous }\end{array}$ & $\begin{array}{c}\text { Irre- } \\
\text { usable }\end{array}$ & & & Created & Reused & Rebuilt & $\begin{array}{l}\text { Parse } \\
\text { Node }\end{array}$ & $\begin{array}{c}\text { Character } \\
\text { Node }\end{array}$ & Count & $\begin{array}{l}\text { Contains } \\
\text { Change }\end{array}$ & $\begin{array}{c}\text { Irre- } \\
\text { usable }\end{array}$ & $\begin{array}{c}\text { No } \\
\text { Actions }\end{array}$ & $\begin{array}{l}\text { Wrong } \\
\text { State }\end{array}$ \\
\hline Average & 1453416 & $0.00 \%$ & $19.08 \%$ & 852068 & Average & 13989 & $99.60 \%$ & $0.08 \%$ & 631 & 1849 & $0.17 \%$ & $51.35 \%$ & $46.67 \%$ & $0.00 \%$ & $1.98 \%$ \\
\hline StringUtils & 572516 & $0.00 \%$ & $15.27 \%$ & 396264 & StringUtils & 8186 & $99.65 \%$ & $0.14 \%$ & 607 & 826 & $0.28 \%$ & $47.76 \%$ & $51.46 \%$ & $0.00 \%$ & $0.78 \%$ \\
\hline gson & 2304220 & $0.00 \%$ & $23.36 \%$ & 1264778 & gson & 7883 & $99.93 \%$ & $0.02 \%$ & 534 & 907 & $0.05 \%$ & $53.68 \%$ & $45.10 \%$ & $0.00 \%$ & $1.22 \%$ \\
\hline slf $4 j$ & 1483511 & $0.00 \%$ & $18.61 \%$ & 895163 & slf $4 j$ & 25897 & $99.21 \%$ & $0.07 \%$ & 752 & 3814 & $0.19 \%$ & $52.63 \%$ & $43.44 \%$ & $0.00 \%$ & $3.93 \%$ \\
\hline
\end{tabular}


Incremental parsing measurements for Java source StringUtils.

\begin{tabular}{|c|c|c|c|c|c|c|c|c|c|c|c|c|c|c|c|}
\hline \multirow[b]{2}{*}{ Version } & \multicolumn{3}{|c|}{ Parse Nodes } & \multirow{2}{*}{$\begin{array}{c}\text { Character } \\
\text { Nodes } \\
\text { Count }\end{array}$} & \multirow[b]{2}{*}{ Version } & \multicolumn{3}{|c|}{ Parse Nodes } & \multicolumn{2}{|c|}{ Shift } & \multicolumn{5}{|c|}{ Breakdown } \\
\hline & Count & $\begin{array}{l}\text { Ambi- } \\
\text { guous }\end{array}$ & $\begin{array}{c}\text { Irre- } \\
\text { usable }\end{array}$ & & & Created & Reused & Rebuilt & $\begin{array}{l}\text { Parse } \\
\text { Node }\end{array}$ & $\begin{array}{c}\text { Character } \\
\text { Node }\end{array}$ & Count & $\begin{array}{l}\text { Contains } \\
\text { Change }\end{array}$ & $\begin{array}{c}\text { Irre- } \\
\text { usable }\end{array}$ & $\begin{array}{c}\text { No } \\
\text { Actions }\end{array}$ & $\begin{array}{l}\text { Wrong } \\
\text { State }\end{array}$ \\
\hline $\begin{array}{c}\text { Average } \\
(0 . .14)\end{array}$ & 572516 & $\begin{array}{r}0 \\
(0.00 \%)\end{array}$ & $\begin{array}{r}87442 \\
(15.27 \%)\end{array}$ & 396264 & $\begin{array}{c}\text { Average } \\
(1 . .15)\end{array}$ & 8186 & $\begin{array}{r}570514 \\
(99.65 \%) \\
\end{array}$ & $\begin{array}{r}802 \\
(0.14 \%) \\
\end{array}$ & 607 & 826 & $\begin{array}{r}1585 \\
(0.28 \%)\end{array}$ & $\begin{array}{r}809 \\
(47.76 \%) \\
\end{array}$ & $\begin{array}{r}745 \\
(51.46 \%) \\
\end{array}$ & $\begin{array}{r}0 \\
(0.00 \%)\end{array}$ & $\begin{array}{r}31 \\
(0.78 \%) \\
\end{array}$ \\
\hline 0 & 572123 & $\begin{array}{r}0 \\
(0.00 \%)\end{array}$ & $\begin{array}{r}87583 \\
(15.31 \%)\end{array}$ & 395110 & $\begin{aligned} & \rightarrow 0 \\
0 & \rightarrow 1\end{aligned}$ & $\begin{array}{r}1058237 \\
4125\end{array}$ & $\begin{array}{r}571624 \\
(99.91 \%)\end{array}$ & $\begin{array}{r}269 \\
(0.05 \%)\end{array}$ & $\begin{array}{r}0 \\
463\end{array}$ & $\begin{array}{r}395111 \\
86\end{array}$ & $\begin{array}{r}498 \\
(0.09 \%)\end{array}$ & $\begin{array}{r}225 \\
(45.18 \%)\end{array}$ & $\begin{array}{r}273 \\
(54.82 \%)\end{array}$ & $\begin{array}{r}0 \\
(0.00 \%)\end{array}$ & $\begin{array}{r}0 \\
(0.00 \%)\end{array}$ \\
\hline 1 & 572280 & $\begin{array}{r}0 \\
(0.00 \%)\end{array}$ & $\begin{array}{r}87611 \\
(15.31 \%)\end{array}$ & 395172 & $1 \rightarrow 2$ & 3430 & $\begin{array}{r}571811 \\
(99.92 \%)\end{array}$ & $\begin{array}{r}294 \\
(0.05 \%)\end{array}$ & 307 & 139 & $\begin{array}{r}468 \\
(0.08 \%)\end{array}$ & $\begin{array}{r}146 \\
(31.20 \%)\end{array}$ & $\begin{array}{r}318 \\
(67.95 \%)\end{array}$ & $\begin{array}{r}0 \\
(0.00 \%)\end{array}$ & $\begin{array}{r}4 \\
(0.85 \%)\end{array}$ \\
\hline 2 & 572360 & $\begin{array}{r}0 \\
(0.00 \%)\end{array}$ & $\begin{array}{r}87639 \\
(15.31 \%)\end{array}$ & 395216 & $2 \rightarrow 3$ & 10379 & $\begin{array}{r}569573 \\
(99.51 \%)\end{array}$ & $\begin{array}{r}1187 \\
(0.21 \%)\end{array}$ & 864 & 643 & $\begin{array}{r}1975 \\
(0.35 \%)\end{array}$ & $\begin{array}{r}815 \\
(41.27 \%)\end{array}$ & $\begin{array}{r}1136 \\
(57.52 \%)\end{array}$ & $\begin{array}{r}0 \\
(0.00 \%)\end{array}$ & $\begin{array}{r}24 \\
(1.22 \%)\end{array}$ \\
\hline 3 & 571559 & $\begin{array}{r}0 \\
(0.00 \%)\end{array}$ & $\begin{array}{r}87419 \\
(15.29 \%)\end{array}$ & 394856 & $3 \rightarrow 4$ & 10617 & $\begin{array}{r}571140 \\
(99.93 \%)\end{array}$ & $\begin{array}{r}207 \\
(0.04 \%)\end{array}$ & 345 & 2829 & $\begin{array}{r}418 \\
(0.07 \%)\end{array}$ & $\begin{array}{r}214 \\
(51.20 \%)\end{array}$ & $\begin{array}{r}203 \\
(48.56 \%)\end{array}$ & $\begin{array}{r}0 \\
(0.00 \%)\end{array}$ & $\begin{array}{r}1 \\
(0.24 \%)\end{array}$ \\
\hline 4 & 575679 & $\begin{array}{r}0 \\
(0.00 \%)\end{array}$ & $\begin{array}{r}88130 \\
(15.31 \%)\end{array}$ & 397653 & $4 \rightarrow 5$ & 38596 & $\begin{array}{r}560927 \\
(97.44 \%)\end{array}$ & $\begin{array}{r}4185 \\
(0.73 \%)\end{array}$ & 2107 & 5078 & $\begin{array}{r}11288 \\
(1.96 \%)\end{array}$ & $\begin{array}{r}7415 \\
(65.69 \%)\end{array}$ & $\begin{array}{r}3609 \\
(31.97 \%)\end{array}$ & $\begin{array}{r}0 \\
(0.00 \%)\end{array}$ & $\begin{array}{r}264 \\
(2.34 \%)\end{array}$ \\
\hline 5 & 571608 & $\begin{array}{r}0 \\
(0.00 \%)\end{array}$ & $\begin{array}{r}87203 \\
(15.26 \%)\end{array}$ & 395931 & $5 \rightarrow 6$ & 52 & $\begin{array}{r}571537 \\
(99.99 \%)\end{array}$ & $\begin{array}{r}12 \\
(0.00 \%)\end{array}$ & 9 & 2 & $\begin{array}{r}17 \\
(0.00 \%)\end{array}$ & $\begin{array}{r}14 \\
(82.35 \%)\end{array}$ & $\begin{array}{r}3 \\
(17.65 \%)\end{array}$ & $\begin{array}{r}0 \\
(0.00 \%)\end{array}$ & $\begin{array}{r}0 \\
(0.00 \%)\end{array}$ \\
\hline 6 & 571549 & $\begin{array}{r}0 \\
(0.00 \%)\end{array}$ & $\begin{array}{r}87192 \\
(15.26 \%)\end{array}$ & 395896 & $6 \rightarrow 7$ & 2400 & $\begin{array}{r}570976 \\
(99.90 \%)\end{array}$ & $\begin{array}{r}250 \\
(0.04 \%)\end{array}$ & 257 & 81 & $\begin{array}{r}464 \\
(0.08 \%)\end{array}$ & $\begin{array}{r}245 \\
(52.80 \%)\end{array}$ & $\begin{array}{r}218 \\
(46.98 \%)\end{array}$ & $\begin{array}{r}0 \\
(0.00 \%)\end{array}$ & $\begin{array}{r}1 \\
(0.22 \%)\end{array}$ \\
\hline 7 & 571380 & $\begin{array}{r}0 \\
(0.00 \%)\end{array}$ & $\begin{array}{r}87129 \\
(15.25 \%)\end{array}$ & 395842 & $7 \rightarrow 8$ & 3306 & $\begin{array}{r}570856 \\
(99.91 \%)\end{array}$ & $\begin{array}{r}323 \\
(0.06 \%)\end{array}$ & 358 & 84 & $\begin{array}{r}515 \\
(0.09 \%)\end{array}$ & $\begin{array}{r}149 \\
(28.93 \%)\end{array}$ & $\begin{array}{r}362 \\
(70.29 \%)\end{array}$ & $\begin{array}{r}0 \\
(0.00 \%)\end{array}$ & $\begin{array}{r}4 \\
(0.78 \%)\end{array}$ \\
\hline 8 & 571370 & $\begin{array}{r}0 \\
(0.00 \%)\end{array}$ & $\begin{array}{r}87120 \\
(15.25 \%)\end{array}$ & 395842 & $8 \rightarrow 9$ & 4113 & $\begin{array}{r}571080 \\
(99.95 \%)\end{array}$ & $\begin{array}{r}120 \\
(0.02 \%)\end{array}$ & 113 & 1415 & $\begin{array}{r}289 \\
(0.05 \%)\end{array}$ & $\begin{array}{r}171 \\
(59.17 \%)\end{array}$ & $\begin{array}{r}117 \\
(40.48 \%)\end{array}$ & $\begin{array}{r}0 \\
(0.00 \%)\end{array}$ & $\begin{array}{r}1 \\
(0.35 \%)\end{array}$ \\
\hline 9 & 573253 & $\begin{array}{r}0 \\
(0.00 \%)\end{array}$ & $\begin{array}{r}87429 \\
(15.25 \%)\end{array}$ & 397158 & $9 \rightarrow 10$ & 3055 & $\begin{array}{r}572754 \\
(99.91 \%)\end{array}$ & $\begin{array}{r}288 \\
(0.05 \%)\end{array}$ & 372 & 81 & $\begin{array}{r}498 \\
(0.09 \%)\end{array}$ & $\begin{array}{r}180 \\
(36.14 \%)\end{array}$ & $\begin{array}{r}311 \\
(62.45 \%)\end{array}$ & $\begin{array}{r}0 \\
(0.00 \%)\end{array}$ & $\begin{array}{r}7 \\
(1.41 \%)\end{array}$ \\
\hline 10 & 573271 & $\begin{array}{r}0 \\
(0.00 \%)\end{array}$ & $\begin{array}{r}87435 \\
(15.25 \%)\end{array}$ & 397176 & $10 \rightarrow 11$ & 3824 & $\begin{array}{r}572704 \\
(99.90 \%)\end{array}$ & $\begin{array}{r}253 \\
(0.04 \%)\end{array}$ & 505 & 49 & $\begin{array}{r}566 \\
(0.10 \%)\end{array}$ & $\begin{array}{r}317 \\
(56.01 \%)\end{array}$ & $\begin{array}{r}245 \\
(43.29 \%)\end{array}$ & $\begin{array}{r}0 \\
(0.00 \%)\end{array}$ & $\begin{array}{r}4 \\
(0.71 \%)\end{array}$ \\
\hline 11 & 573279 & $\begin{array}{r}0 \\
(0.00 \%)\end{array}$ & $\begin{array}{r}87435 \\
(15.25 \%)\end{array}$ & 397184 & $11 \rightarrow 12$ & 6150 & $\begin{array}{r}571851 \\
(99.75 \%)\end{array}$ & $\begin{array}{r}943 \\
(0.16 \%)\end{array}$ & 681 & 171 & $\begin{array}{r}1110 \\
(0.19 \%)\end{array}$ & $\begin{array}{r}393 \\
(35.41 \%)\end{array}$ & $\begin{array}{r}714 \\
(64.32 \%)\end{array}$ & $\begin{array}{r}0 \\
(0.00 \%)\end{array}$ & $\begin{array}{r}3 \\
(0.27 \%)\end{array}$ \\
\hline 12 & 572868 & $\begin{array}{r}0 \\
(0.00 \%)\end{array}$ & $\begin{array}{r}87432 \\
(15.26 \%)\end{array}$ & 397048 & $12 \rightarrow 13$ & 1941 & $\begin{array}{r}572391 \\
(99.92 \%)\end{array}$ & $\begin{array}{r}257 \\
(0.04 \%)\end{array}$ & 238 & 114 & $\begin{array}{r}476 \\
(0.08 \%)\end{array}$ & $\begin{array}{r}239 \\
(50.21 \%)\end{array}$ & $\begin{array}{r}236 \\
(49.58 \%)\end{array}$ & $\begin{array}{r}0 \\
(0.00 \%)\end{array}$ & $\begin{array}{r}1 \\
(0.21 \%)\end{array}$ \\
\hline 13 & 572909 & $\begin{array}{r}0 \\
(0.00 \%)\end{array}$ & $\begin{array}{r}87432 \\
(15.26 \%)\end{array}$ & 397077 & $13 \rightarrow 14$ & 26307 & $\begin{array}{r}566933 \\
(98.96 \%)\end{array}$ & $\begin{array}{r}3134 \\
(0.55 \%)\end{array}$ & 2027 & 1457 & $\begin{array}{r}4549 \\
(0.79 \%)\end{array}$ & $\begin{array}{r}1280 \\
(28.14 \%)\end{array}$ & $\begin{array}{r}3125 \\
(68.70 \%)\end{array}$ & $\begin{array}{r}0 \\
(0.00 \%)\end{array}$ & $\begin{array}{r}144 \\
(3.17 \%)\end{array}$ \\
\hline 14 & 572251 & $\begin{array}{r}0 \\
(0.00 \%)\end{array}$ & $\begin{array}{r}87448 \\
(15.28 \%)\end{array}$ & 396792 & $14 \rightarrow 15$ & 4491 & $\begin{array}{r}571559 \\
(99.88 \%)\end{array}$ & $\begin{array}{r}307 \\
(0.05 \%)\end{array}$ & 465 & 158 & $\begin{array}{r}643 \\
(0.11 \%)\end{array}$ & $\begin{array}{r}339 \\
(52.72 \%)\end{array}$ & $\begin{array}{r}304 \\
(47.28 \%)\end{array}$ & $(0.00 \%)$ & $\begin{array}{r}0 \\
(0.00 \%)\end{array}$ \\
\hline 15 & 572267 & $\begin{array}{r}0 \\
(0.00 \%)\end{array}$ & $\begin{array}{r}87424 \\
(15.28 \%)\end{array}$ & 396796 & & & & & & & & & & & \\
\hline
\end{tabular}


Incremental parsing measurements for Java source gson.

\begin{tabular}{|c|c|c|c|c|c|c|c|c|c|c|c|c|c|c|c|}
\hline \multirow[b]{2}{*}{ Version } & \multicolumn{3}{|c|}{ Parse Nodes } & \multirow{2}{*}{$\begin{array}{c}\text { Character } \\
\text { Nodes } \\
\text { Count }\end{array}$} & \multirow[b]{2}{*}{ Version } & \multicolumn{3}{|c|}{ Parse Nodes } & \multicolumn{2}{|c|}{ Shift } & \multicolumn{5}{|c|}{ Breakdown } \\
\hline & Count & $\begin{array}{l}\text { Ambi- } \\
\text { guous }\end{array}$ & $\begin{array}{c}\text { Irre- } \\
\text { usable }\end{array}$ & & & Created & Reused & Rebuilt & $\begin{array}{l}\text { Parse } \\
\text { Node }\end{array}$ & $\begin{array}{c}\text { Character } \\
\text { Node }\end{array}$ & Count & $\begin{array}{l}\text { Contains } \\
\text { Change }\end{array}$ & $\begin{array}{c}\text { Irre- } \\
\text { usable }\end{array}$ & $\begin{array}{c}\text { No } \\
\text { Actions }\end{array}$ & $\begin{array}{l}\text { Wrong } \\
\text { State }\end{array}$ \\
\hline $\begin{array}{c}\text { Average } \\
(0 . .14)\end{array}$ & 2304220 & $\begin{array}{r}0 \\
(0.00 \%) \\
\end{array}$ & $\begin{array}{r}538217 \\
(23.36 \%) \\
\end{array}$ & 1264778 & $\begin{array}{c}\text { Average } \\
(1 . .15)\end{array}$ & 7883 & $\begin{array}{l}2302621 \\
(99.93 \%) \\
\end{array}$ & $\begin{array}{r}430 \\
(0.02 \%) \\
\end{array}$ & 534 & 907 & $\begin{array}{r}1039 \\
(0.05 \%) \\
\end{array}$ & $\begin{array}{r}559 \\
(53.68 \%) \\
\end{array}$ & $\begin{array}{r}459 \\
(45.10 \%)\end{array}$ & $\begin{array}{r}0 \\
(0.00 \%)\end{array}$ & $\begin{array}{r}20 \\
(1.22 \%)\end{array}$ \\
\hline 0 & 2302158 & $\begin{array}{r}0 \\
(000 \%)\end{array}$ & $\begin{array}{r}537864 \\
(2336 \%)\end{array}$ & 1263518 & $\begin{aligned} & \rightarrow 0 \\
0 & \rightarrow 1\end{aligned}$ & $\begin{array}{r}6362523 \\
7120\end{array}$ & $\begin{array}{l}2299996 \\
(9991 \%)\end{array}$ & $\begin{array}{r}457 \\
(0,02 \%)\end{array}$ & $\begin{array}{r}0 \\
580\end{array}$ & $\begin{array}{r}1263722 \\
709\end{array}$ & $\begin{array}{r}1622 \\
(0.07 \%)\end{array}$ & $\begin{array}{r}1002 \\
(6178 \%\end{array}$ & $\begin{array}{r}560 \\
(3453 \%)\end{array}$ & $\begin{array}{r}0 \\
(000 \%)\end{array}$ & $\begin{array}{r}60 \\
(370 \%)\end{array}$ \\
\hline 1 & 2301731 & $\begin{array}{r}(0.00 \%) \\
0 \\
(0.00 \%)\end{array}$ & $\begin{array}{r}(23.36 \%) \\
537769 \\
(23.36 \%)\end{array}$ & 1263366 & $1 \rightarrow 2$ & 2253 & $\begin{array}{l}(99.91 \%) \\
2301180 \\
(99.98 \%)\end{array}$ & $\begin{array}{r}(0.02 \%) \\
270 \\
(0.01 \%)\end{array}$ & 346 & 182 & $\begin{array}{r}(0.07 \%) \\
477 \\
(0.02 \%)\end{array}$ & $\begin{array}{r}(61.78 \%) \\
214 \\
(44.86 \%)\end{array}$ & $\begin{array}{r}(34.53 \%) \\
232 \\
(48.64 \%)\end{array}$ & $\begin{array}{r}(0.00 \%) \\
0 \\
(0.00 \%)\end{array}$ & $\begin{array}{r}(3.70 \%) \\
31 \\
(6.50 \%)\end{array}$ \\
\hline 2 & 2301561 & $\begin{array}{r}0 \\
(0.00 \%)\end{array}$ & $\begin{array}{r}537742 \\
(23.36 \%)\end{array}$ & 1263290 & $2 \rightarrow 3$ & 3267 & $\begin{array}{l}2300876 \\
(99.97 \%)\end{array}$ & $\begin{array}{r}333 \\
(0.01 \%)\end{array}$ & 494 & 144 & $\begin{array}{r}701 \\
(0.03 \%)\end{array}$ & $\begin{array}{r}287 \\
(40.94 \%)\end{array}$ & $\begin{array}{r}410 \\
(58.49 \%)\end{array}$ & $\begin{array}{r}0 \\
(0.00 \%)\end{array}$ & $\begin{array}{r}4 \\
(0.57 \%)\end{array}$ \\
\hline 3 & 2301512 & $\begin{array}{r}0 \\
(0.00 \%)\end{array}$ & $\begin{array}{r}537746 \\
(23.36 \%)\end{array}$ & 1263286 & $3 \rightarrow 4$ & 36200 & $\begin{array}{l}2293631 \\
(99.66 \%)\end{array}$ & $\begin{array}{r}2972 \\
(0.13 \%)\end{array}$ & 2755 & 1742 & $\begin{array}{r}6093 \\
(0.26 \%)\end{array}$ & $\begin{array}{r}2519 \\
(41.34 \%)\end{array}$ & $\begin{array}{r}3408 \\
(55.93 \%)\end{array}$ & $\begin{array}{r}0 \\
(0.00 \%)\end{array}$ & $\begin{array}{r}166 \\
(2.72 \%)\end{array}$ \\
\hline 4 & 2300237 & $\begin{array}{r}0 \\
(000 \%)\end{array}$ & 537100 & 1262874 & $4 \rightarrow 5$ & 0 & & & 204 & 0 & & & & & \\
\hline & & $\begin{array}{r}(0.00 \%) \\
0\end{array}$ & $\begin{array}{r}(23.35 \%) \\
537100\end{array}$ & & $5 \rightarrow 6$ & 1504 & $\begin{array}{l}2300069 \\
(99.99 \%)\end{array}$ & $\begin{array}{r}74 \\
(0.00 \%)\end{array}$ & 309 & 160 & $\begin{array}{r}167 \\
(0.01 \%)\end{array}$ & $\begin{array}{r}61 \\
(36.53 \%)\end{array}$ & $\begin{array}{r}106 \\
(63.47 \%)\end{array}$ & $\begin{array}{r}0 \\
(0.00 \%)\end{array}$ & $\begin{array}{r}0 \\
(0.00 \%)\end{array}$ \\
\hline 5 & 2300237 & $\begin{array}{r}(0.00 \%) \\
0\end{array}$ & $\begin{array}{r}(23.35 \%) \\
537130\end{array}$ & 1262874 & $6 \rightarrow 7$ & 3474 & $\begin{array}{l}2299610 \\
(99.96 \%)\end{array}$ & $\begin{array}{r}260 \\
(0.01 \%)\end{array}$ & 442 & 161 & $\begin{array}{r}442 \\
(0.02 \%)\end{array}$ & $\begin{array}{r}304 \\
(68.78 \%)\end{array}$ & $\begin{array}{r}134 \\
(30.32 \%)\end{array}$ & $\begin{array}{r}0 \\
(0.00 \%)\end{array}$ & $\begin{array}{r}4 \\
(0.90 \%)\end{array}$ \\
\hline 6 & 2300455 & $\begin{array}{r}(0.00 \%) \\
0\end{array}$ & $\begin{array}{r}(23.35 \%) \\
537170\end{array}$ & 1263000 & $7 \rightarrow 8$ & 514 & $\begin{array}{r}2300290 \\
(100.00 \%)\end{array}$ & $\begin{array}{r}7 \\
(0.00 \%)\end{array}$ & 213 & 377 & $\begin{array}{r}13 \\
(0.00 \%)\end{array}$ & $\begin{array}{r}9 \\
(69.23 \%)\end{array}$ & $\begin{array}{r}4 \\
(30.77 \%)\end{array}$ & $\begin{array}{r}0 \\
(0.00 \%)\end{array}$ & $\begin{array}{r}0 \\
(0.00 \%)\end{array}$ \\
\hline 7 & 2300304 & $(0.00 \%)$ & $(23.35 \%)$ & 1262824 & & & 2297824 & 876 & & & 2943 & 2099 & 832 & $\begin{array}{r}(0.007) \\
0\end{array}$ & 12 \\
\hline 8 & 2300750 & $\begin{array}{r}0 \\
(0.00 \%)\end{array}$ & $\begin{array}{r}537218 \\
(23.35 \%)\end{array}$ & 1263198 & $8 \rightarrow 9$ & 3867 & $\begin{array}{l}(99.87 \%) \\
2300657\end{array}$ & $\begin{array}{r}(0.04 \%) \\
84\end{array}$ & 585 & 1741 & $\begin{array}{r}(0.13 \%) \\
169\end{array}$ & $\begin{array}{r}(71.32 \%) \\
83\end{array}$ & $\begin{array}{r}(28.27 \%) \\
86\end{array}$ & $\begin{array}{r}(0.00 \%) \\
0\end{array}$ & $\begin{array}{r}(0.41 \%) \\
0\end{array}$ \\
\hline 9 & 2300828 & $\begin{array}{r}0 \\
(0.00 \%)\end{array}$ & $\begin{array}{r}537218 \\
(23.35 \%)\end{array}$ & 1263276 & $\rightarrow 10$ & 31772 & $\begin{array}{l}(99.99 \%) \\
2310474\end{array}$ & $\begin{array}{r}(0.00 \%) \\
30\end{array}$ & 321 & 4648 & $\begin{array}{r}(0.01 \%) \\
62\end{array}$ & $\begin{array}{r}(49.11 \%) \\
34\end{array}$ & $\begin{array}{r}(50.89 \%) \\
28\end{array}$ & $\begin{array}{r}(0.00 \%) \\
0\end{array}$ & $\begin{array}{r}(0.00 \%) \\
0\end{array}$ \\
\hline 10 & 2310537 & $\begin{array}{r}0 \\
(0.00 \%)\end{array}$ & $\begin{array}{r}539822 \\
(2336 \%)\end{array}$ & 1267874 & $\rightarrow 11$ & 950 & $(100.00 \%)$ & $(0.00 \%)$ & 268 & 166 & $\begin{array}{r}(0.00 \%) \\
\end{array}$ & $(54.84 \%)$ & $(45.16 \%)$ & $(0.00 \%)$ & $(0.00 \%)$ \\
\hline 11 & 2330798 & & $\begin{array}{r}(20.36 \%) \\
539875\end{array}$ & 1268038 & $11 \rightarrow 12$ & 1192 & $\begin{array}{l}2310659 \\
(99.99 \%)\end{array}$ & $\begin{array}{r}84 \\
(0.00 \%)\end{array}$ & 296 & 102 & $\begin{array}{r}138 \\
(0.01 \%)\end{array}$ & $\begin{array}{r}32 \\
(23.19 \%)\end{array}$ & $\begin{array}{r}106 \\
(76.81 \%)\end{array}$ & $(0.00 \%)$ & $(0.00 \%)$ \\
\hline 11 & 2310798 & $\begin{array}{r}(0.00 \%) \\
0\end{array}$ & $\begin{array}{r}(23.36 \%) \\
539913\end{array}$ & 126803 & $12 \rightarrow 13$ & 7289 & $\begin{array}{l}2307965 \\
(99.87 \%)\end{array}$ & $\begin{array}{r}304 \\
(0.01 \%)\end{array}$ & 375 & 866 & $\begin{array}{r}805 \\
(0.03 \%)\end{array}$ & $\begin{array}{r}500 \\
(62.11 \%)\end{array}$ & $\begin{array}{r}299 \\
(37.14 \%)\end{array}$ & $\begin{array}{r}0 \\
(0.00 \%)\end{array}$ & $\begin{array}{r}6 \\
(0.75 \%)\end{array}$ \\
\hline 12 & 2310965 & $\begin{array}{r}(0.00 \%) \\
0\end{array}$ & $\begin{array}{r}(23.36 \%) \\
539656\end{array}$ & 126812 & $13 \rightarrow 14$ & 11558 & $\begin{array}{l}2307585 \\
(99.90 \%)\end{array}$ & $\begin{array}{r}397 \\
(0.02 \%)\end{array}$ & 441 & 1737 & $\begin{array}{r}1145 \\
(0.05 \%)\end{array}$ & $\begin{array}{r}748 \\
(65.33 \%)\end{array}$ & $\begin{array}{r}388 \\
(33.89 \%)\end{array}$ & $\begin{array}{r}0 \\
(0.00 \%)\end{array}$ & $\begin{array}{r}9 \\
(0.79 \%)\end{array}$ \\
\hline 13 & 2309965 & $(0.00 \%)$ & $(23.36 \%)$ & 1267740 & & & 2308257 & 304 & 375 & 866 & 805 & 500 & 299 & & \\
\hline 14 & 2311257 & $\begin{array}{r}0 \\
(0.00 \%)\end{array}$ & $\begin{array}{r}539929 \\
(23.36 \%)\end{array}$ & 1268390 & 10 & 1789 & $(99.87 \%)$ & $(0.01 \%)$ & 3/3 & 806 & $(0.03 \%)$ & $(62.11 \%)$ & $(37.14 \%)$ & $(0.00 \%)$ & $(0.75 \%)$ \\
\hline 15 & 2310257 & $\begin{array}{r}0 \\
(0.00 \%)\end{array}$ & $\begin{array}{r}539672 \\
(23.36 \%)\end{array}$ & 1268008 & & & & & & & & & & & \\
\hline
\end{tabular}


Incremental parsing measurements for Java source slf4j.

\begin{tabular}{|c|c|c|c|c|c|c|c|c|c|c|c|c|c|c|c|}
\hline \multirow[b]{2}{*}{ Version } & \multicolumn{3}{|c|}{ Parse Nodes } & \multirow{2}{*}{$\begin{array}{c}\text { Character } \\
\text { Nodes } \\
\text { Count } \\
\end{array}$} & \multirow[b]{2}{*}{ Version } & \multicolumn{3}{|c|}{ Parse Nodes } & \multicolumn{2}{|c|}{ Shift } & \multicolumn{5}{|c|}{ Breakdown } \\
\hline & Count & $\begin{array}{l}\text { Ambi- } \\
\text { guous }\end{array}$ & $\begin{array}{c}\text { Irre- } \\
\text { usable }\end{array}$ & & & Created & Reused & Rebuilt & $\begin{array}{l}\text { Parse } \\
\text { Node }\end{array}$ & $\begin{array}{c}\text { Character } \\
\text { Node }\end{array}$ & Count & $\begin{array}{l}\text { Contains } \\
\text { Change }\end{array}$ & $\begin{array}{c}\text { Irre- } \\
\text { usable }\end{array}$ & $\begin{array}{c}\text { No } \\
\text { Actions }\end{array}$ & $\begin{array}{l}\text { Wrong } \\
\text { State }\end{array}$ \\
\hline $\begin{array}{c}\text { Average } \\
(0 . .14)\end{array}$ & 1483511 & $\begin{array}{r}0 \\
(0.00 \%)\end{array}$ & $\begin{array}{r}276101 \\
(18.61 \%)\end{array}$ & 895163 & $\begin{array}{c}\text { Average } \\
(1 . .15)\end{array}$ & 25897 & $\begin{array}{l}1471637 \\
(99.21 \%)\end{array}$ & $\begin{array}{r}1053 \\
(0.07 \%)\end{array}$ & 752 & 3814 & $\begin{array}{r}2918 \\
(0.19 \%)\end{array}$ & $\begin{array}{r}1936 \\
(52.63 \%)\end{array}$ & $\begin{array}{r}773 \\
(43.44 \%) \\
\end{array}$ & $\begin{array}{r}0 \\
(0.00 \%)\end{array}$ & $\begin{array}{r}209 \\
(3.93 \%) \\
\end{array}$ \\
\hline 0 & 1519876 & $\begin{array}{r}0 \\
(0.00 \%)\end{array}$ & $\begin{array}{r}282515 \\
(18.59 \%)\end{array}$ & 912784 & $\begin{aligned} & \rightarrow 0 \\
0 & \rightarrow 1\end{aligned}$ & $\begin{array}{r}3584556 \\
13481\end{array}$ & $\begin{array}{l}1517062 \\
(99.81 \%)\end{array}$ & $\begin{array}{r}2548 \\
(0.17 \%)\end{array}$ & $\begin{array}{r}0 \\
1486\end{array}$ & $\begin{array}{r}913020 \\
1074\end{array}$ & $\begin{array}{r}2813 \\
(0.19 \%)\end{array}$ & $\begin{array}{r}9 \\
(0.32 \%)\end{array}$ & $\begin{array}{r}2384 \\
(84.75 \%)\end{array}$ & $\begin{array}{r}0 \\
(0.00 \%)\end{array}$ & $\begin{array}{r}420 \\
(14.93 \%)\end{array}$ \\
\hline 1 & 1519878 & $\begin{array}{r}0 \\
(0.00 \%)\end{array}$ & $\begin{array}{r}282515 \\
(18.59 \%)\end{array}$ & 912785 & $1 \rightarrow 2$ & 320 & $\begin{array}{l}1519682 \\
(99.99 \%)\end{array}$ & $\begin{array}{r}74 \\
(0.00 \%)\end{array}$ & 274 & 175 & $\begin{array}{r}203 \\
(0.01 \%)\end{array}$ & $\begin{array}{r}181 \\
(89.16 \%)\end{array}$ & $\begin{array}{r}21 \\
(10.34 \%)\end{array}$ & $\begin{array}{r}0 \\
(0.00 \%)\end{array}$ & $\begin{array}{r}1 \\
(0.49 \%)\end{array}$ \\
\hline 2 & 1519912 & $\begin{array}{r}0 \\
(0.00 \%)\end{array}$ & $\begin{array}{r}282519 \\
(18.59 \%)\end{array}$ & 912813 & $2 \rightarrow 3$ & 1626 & $\begin{array}{l}1519648 \\
(99.98 \%)\end{array}$ & $\begin{array}{r}160 \\
(0.01 \%)\end{array}$ & 451 & 40 & $\begin{array}{r}263 \\
(0.02 \%)\end{array}$ & $\begin{array}{r}81 \\
(30.80 \%)\end{array}$ & $\begin{array}{r}178 \\
(67.68 \%)\end{array}$ & $\begin{array}{r}0 \\
(0.00 \%)\end{array}$ & $\begin{array}{r}4 \\
(1.52 \%)\end{array}$ \\
\hline 3 & 1519914 & $\begin{array}{r}0 \\
(0.00 \%)\end{array}$ & $\begin{array}{r}282518 \\
(18.59 \%)\end{array}$ & 912814 & $3 \rightarrow 4$ & 62643 & $\begin{array}{l}1499575 \\
(98.66 \%)\end{array}$ & $\begin{array}{r}604 \\
(0.04 \%)\end{array}$ & 639 & 10153 & $\begin{array}{r}1719 \\
(0.11 \%)\end{array}$ & $\begin{array}{r}1134 \\
(65.97 \%)\end{array}$ & $\begin{array}{r}533 \\
(31.01 \%)\end{array}$ & $\begin{array}{r}0 \\
(0.00 \%)\end{array}$ & $\begin{array}{r}52 \\
(3.03 \%)\end{array}$ \\
\hline 4 & 1520978 & $\begin{array}{r}0 \\
(0.00 \%)\end{array}$ & $\begin{array}{r}282566 \\
(18.58 \%)\end{array}$ & 913852 & $4 \rightarrow 5$ & 121190 & $\begin{array}{l}1466834 \\
(96.44 \%)\end{array}$ & $\begin{array}{r}5295 \\
(0.35 \%)\end{array}$ & 2698 & 12787 & $\begin{array}{r}16988 \\
(1.12 \%)\end{array}$ & $\begin{array}{r}12645 \\
(74.43 \%)\end{array}$ & $\begin{array}{r}3903 \\
(22.98 \%)\end{array}$ & $\begin{array}{r}0 \\
(0.00 \%)\end{array}$ & $\begin{array}{r}440 \\
(2.59 \%)\end{array}$ \\
\hline 5 & 1496179 & $\begin{array}{r}0 \\
(0.00 \%)\end{array}$ & $\begin{array}{r}278176 \\
(18.59 \%)\end{array}$ & 902914 & $5 \rightarrow 6$ & 1012 & $\begin{array}{l}1495997 \\
(99.99 \%)\end{array}$ & $\begin{array}{r}80 \\
(0.01 \%)\end{array}$ & 339 & 42 & $\begin{array}{r}187 \\
(0.01 \%)\end{array}$ & $\begin{array}{r}86 \\
(45.99 \%)\end{array}$ & $\begin{array}{r}99 \\
(52.94 \%)\end{array}$ & $\begin{array}{r}0 \\
(0.00 \%)\end{array}$ & $\begin{array}{r}2 \\
(1.07 \%)\end{array}$ \\
\hline 6 & 1496177 & $\begin{array}{r}0 \\
(0.00 \%)\end{array}$ & $\begin{array}{r}278176 \\
(18.59 \%)\end{array}$ & 902912 & $6 \rightarrow 7$ & 125435 & $\begin{array}{l}1422510 \\
(95.08 \%)\end{array}$ & $\begin{array}{r}2978 \\
(0.20 \%)\end{array}$ & 1278 & 21699 & $\begin{array}{r}10631 \\
(0.71 \%)\end{array}$ & $\begin{array}{r}7981 \\
(75.07 \%)\end{array}$ & $\begin{array}{r}1693 \\
(15.93 \%)\end{array}$ & $\begin{array}{r}0 \\
(0.00 \%)\end{array}$ & $\begin{array}{r}957 \\
(9.00 \%)\end{array}$ \\
\hline 7 & 1464481 & $\begin{array}{r}0 \\
(0.00 \%)\end{array}$ & $\begin{array}{r}272295 \\
(18.59 \%)\end{array}$ & 885244 & $7 \rightarrow 8$ & 46590 & $\begin{array}{l}1440557 \\
(98.37 \%)\end{array}$ & $\begin{array}{r}3243 \\
(0.22 \%)\end{array}$ & 1579 & 7967 & $\begin{array}{r}9335 \\
(0.64 \%)\end{array}$ & $\begin{array}{r}6035 \\
(64.65 \%)\end{array}$ & $\begin{array}{r}2065 \\
(22.12 \%)\end{array}$ & $\begin{array}{r}0 \\
(0.00 \%)\end{array}$ & $\begin{array}{r}1235 \\
(13.23 \%)\end{array}$ \\
\hline 8 & 1455828 & $\begin{array}{r}0 \\
(0.00 \%)\end{array}$ & $\begin{array}{r}271349 \\
(18.64 \%)\end{array}$ & 881191 & $8 \rightarrow 9$ & 740 & $\begin{array}{l}1455669 \\
(99.99 \%)\end{array}$ & $\begin{array}{r}107 \\
(0.01 \%)\end{array}$ & 334 & 33 & $\begin{array}{r}144 \\
(0.01 \%)\end{array}$ & $\begin{array}{r}47 \\
(32.64 \%)\end{array}$ & $\begin{array}{r}94 \\
(65.28 \%)\end{array}$ & $\begin{array}{r}0 \\
(0.00 \%)\end{array}$ & $\begin{array}{r}3 \\
(2.08 \%)\end{array}$ \\
\hline 9 & 1455801 & $\begin{array}{r}0 \\
(0.00 \%)\end{array}$ & $\begin{array}{r}271330 \\
(18.64 \%)\end{array}$ & 881184 & $9 \rightarrow 10$ & 431 & $\begin{array}{l}1455639 \\
(99.99 \%)\end{array}$ & $\begin{array}{r}70 \\
(0.00 \%)\end{array}$ & 303 & 11 & $\begin{array}{r}84 \\
(0.01 \%)\end{array}$ & $\begin{array}{r}47 \\
(55.95 \%)\end{array}$ & $\begin{array}{r}35 \\
(41.67 \%)\end{array}$ & $\begin{array}{r}0 \\
(0.00 \%)\end{array}$ & $\begin{array}{r}2 \\
(2.38 \%)\end{array}$ \\
\hline 10 & 1455715 & $\begin{array}{r}0 \\
(0.00 \%)\end{array}$ & $\begin{array}{r}271317 \\
(18.64 \%)\end{array}$ & 881132 & $10 \rightarrow 11$ & 977 & $\begin{array}{l}1455512 \\
(99.99 \%)\end{array}$ & $\begin{array}{r}103 \\
(0.01 \%)\end{array}$ & 406 & 17 & $\begin{array}{r}197 \\
(0.01 \%)\end{array}$ & $\begin{array}{r}98 \\
(49.75 \%)\end{array}$ & $\begin{array}{r}99 \\
(50.25 \%)\end{array}$ & $\begin{array}{r}0 \\
(0.00 \%)\end{array}$ & $\begin{array}{r}0 \\
(0.00 \%)\end{array}$ \\
\hline 11 & 1455707 & $\begin{array}{r}0 \\
(0.00 \%)\end{array}$ & $\begin{array}{r}271322 \\
(18.64 \%)\end{array}$ & 881134 & $11 \rightarrow 12$ & 3895 & $\begin{array}{l}1455364 \\
(99.98 \%)\end{array}$ & $\begin{array}{r}179 \\
(0.01 \%)\end{array}$ & 381 & 494 & $\begin{array}{r}350 \\
(0.02 \%)\end{array}$ & $\begin{array}{r}155 \\
(44.29 \%)\end{array}$ & $\begin{array}{r}188 \\
(53.71 \%)\end{array}$ & $\begin{array}{r}0 \\
(0.00 \%)\end{array}$ & $\begin{array}{r}7 \\
(2.00 \%)\end{array}$ \\
\hline 12 & 1456442 & $\begin{array}{r}0 \\
(0.00 \%)\end{array}$ & $\begin{array}{r}271509 \\
(18.64 \%)\end{array}$ & 881518 & $12 \rightarrow 13$ & 281 & $\begin{array}{r}1456396 \\
(100.00 \%)\end{array}$ & $\begin{array}{r}30 \\
(0.00 \%)\end{array}$ & 265 & 112 & $\begin{array}{r}43 \\
(0.00 \%)\end{array}$ & $\begin{array}{r}20 \\
(46.51 \%)\end{array}$ & $\begin{array}{r}21 \\
(48.84 \%)\end{array}$ & $\begin{array}{r}0 \\
(0.00 \%)\end{array}$ & $\begin{array}{r}2 \\
(4.65 \%)\end{array}$ \\
\hline 13 & 1456563 & $\begin{array}{r}0 \\
(0.00 \%)\end{array}$ & $\begin{array}{r}271522 \\
(18.64 \%)\end{array}$ & 881618 & $13 \rightarrow 14$ & 7226 & $\begin{array}{l}1455937 \\
(99.96 \%)\end{array}$ & $\begin{array}{r}137 \\
(0.01 \%)\end{array}$ & 404 & 2192 & $\begin{array}{r}247 \\
(0.02 \%)\end{array}$ & $\begin{array}{r}97 \\
(39.27 \%)\end{array}$ & $\begin{array}{r}148 \\
(59.92 \%)\end{array}$ & $\begin{array}{r}0 \\
(0.00 \%)\end{array}$ & $\begin{array}{r}2 \\
(0.81 \%)\end{array}$ \\
\hline 14 & 1459217 & $\begin{array}{r}0 \\
(0.00 \%)\end{array}$ & $\begin{array}{r}271885 \\
(18.63 \%)\end{array}$ & 883547 & $14 \rightarrow 15$ & 2602 & $\begin{array}{l}1458180 \\
(99.93 \%)\end{array}$ & $\begin{array}{r}190 \\
(0.01 \%)\end{array}$ & 445 & 407 & $\begin{array}{r}567 \\
(0.04 \%)\end{array}$ & $\begin{array}{r}423 \\
(74.60 \%)\end{array}$ & $\begin{array}{r}137 \\
(24.16 \%)\end{array}$ & $\begin{array}{r}0 \\
(0.00 \%)\end{array}$ & $\begin{array}{r}7 \\
(1.23 \%)\end{array}$ \\
\hline 15 & 1458960 & $\begin{array}{r}0 \\
(0.00 \%)\end{array}$ & $\begin{array}{r}271819 \\
(18.63 \%)\end{array}$ & 883490 & & & & & & & & & & & \\
\hline
\end{tabular}




\section{$\stackrel{\infty}{\sim}$ A.1.2 WebDSL}

Incremental parsing measurements for the WebDSL language.

\begin{tabular}{|c|c|c|c|c|c|c|c|c|c|c|c|c|c|c|c|}
\hline \multirow[b]{2}{*}{ Source } & \multicolumn{3}{|c|}{ Parse Nodes } & \multirow{2}{*}{$\begin{array}{c}\text { Character } \\
\text { Nodes } \\
\text { Count }\end{array}$} & \multirow[b]{2}{*}{ Source } & \multicolumn{3}{|c|}{ Parse Nodes } & \multicolumn{2}{|c|}{ Shift } & \multicolumn{5}{|c|}{ Breakdown } \\
\hline & Count & $\begin{array}{l}\text { Ambi- } \\
\text { guous }\end{array}$ & $\begin{array}{c}\text { Irre- } \\
\text { usable }\end{array}$ & & & Created & Reused & Rebuilt & $\begin{array}{l}\text { Parse } \\
\text { Node }\end{array}$ & $\begin{array}{l}\text { Character } \\
\text { Node }\end{array}$ & Count & $\begin{array}{l}\text { Contains } \\
\text { Change }\end{array}$ & $\begin{array}{l}\text { Irre- } \\
\text { usable }\end{array}$ & $\begin{array}{c}\text { No } \\
\text { Actions }\end{array}$ & $\begin{array}{c}\text { Wrong } \\
\text { State }\end{array}$ \\
\hline Average & 198475 & $0.00 \%$ & $21.32 \%$ & 101295 & Average & 3733 & $99.55 \%$ & $0.15 \%$ & 324 & 589 & $0.28 \%$ & $51.35 \%$ & $43.76 \%$ & $0.00 \%$ & $4.89 \%$ \\
\hline builtin.app & 182107 & $0.00 \%$ & $23.09 \%$ & 98145 & builtin.app & 2693 & $99.28 \%$ & $0.15 \%$ & 457 & 343 & $0.27 \%$ & $56.16 \%$ & $39.28 \%$ & $0.00 \%$ & $4.56 \%$ \\
\hline YellowGrass & 332492 & $0.00 \%$ & $19.68 \%$ & 166333 & YellowGrass & 6632 & $99.79 \%$ & $0.09 \%$ & 334 & 1220 & $0.17 \%$ & $49.13 \%$ & $46.03 \%$ & $0.00 \%$ & $4.84 \%$ \\
\hline elib-utils & 80827 & $0.00 \%$ & $21.20 \%$ & 39406 & elib-utils & 1874 & $99.57 \%$ & $0.21 \%$ & 181 & 204 & $0.40 \%$ & $48.75 \%$ & $45.97 \%$ & $0.00 \%$ & $5.28 \%$ \\
\hline
\end{tabular}


Incremental parsing measurements for WebDSL source builtin.app.

\begin{tabular}{|c|c|c|c|c|c|c|c|c|c|c|c|c|c|c|c|}
\hline \multirow[b]{2}{*}{ Version } & \multicolumn{3}{|c|}{ Parse Nodes } & \multirow{2}{*}{$\begin{array}{c}\text { Character } \\
\text { Nodes } \\
\text { Count }\end{array}$} & \multirow[b]{2}{*}{ Version } & \multicolumn{3}{|c|}{ Parse Nodes } & \multicolumn{2}{|c|}{ Shift } & \multicolumn{5}{|c|}{ Breakdown } \\
\hline & Count & $\begin{array}{l}\text { Ambi- } \\
\text { guous }\end{array}$ & $\begin{array}{c}\text { Irre- } \\
\text { usable }\end{array}$ & & & Created & Reused & Rebuilt & $\begin{array}{l}\text { Parse } \\
\text { Node }\end{array}$ & $\begin{array}{c}\text { Character } \\
\text { Node }\end{array}$ & Count & $\begin{array}{l}\text { Contains } \\
\text { Change }\end{array}$ & $\begin{array}{c}\text { Irre- } \\
\text { usable }\end{array}$ & $\begin{array}{c}\text { No } \\
\text { Actions }\end{array}$ & $\begin{array}{c}\text { Wrong } \\
\text { State }\end{array}$ \\
\hline $\begin{array}{c}\text { Average } \\
(1 . .9)\end{array}$ & 182107 & $\begin{array}{r}0 \\
(0.00 \%)\end{array}$ & $\begin{array}{r}42046 \\
(23.09 \%)\end{array}$ & 98145 & $\begin{array}{c}\text { Average } \\
(2 . .10)\end{array}$ & 2693 & $\begin{array}{r}180774 \\
(99.28 \%) \\
\end{array}$ & $\begin{array}{r}277 \\
(0.15 \%) \\
\end{array}$ & 457 & 343 & $\begin{array}{r}497 \\
(0.27 \%) \\
\end{array}$ & $\begin{array}{r}222 \\
(56.16 \%)\end{array}$ & $\begin{array}{r}247 \\
(39.28 \%)\end{array}$ & $\begin{array}{r}0 \\
(0.00 \%)\end{array}$ & $\begin{array}{r}28 \\
(4.56 \%)\end{array}$ \\
\hline & & & & & $\rightarrow 1$ & 479900 & & & 0 & 100883 & & & & & \\
\hline 1 & 186944 & $\begin{array}{r}0 \\
(0.00 \%)\end{array}$ & $\begin{array}{r}43193 \\
(23.10 \%)\end{array}$ & 100882 & $1 \rightarrow 2$ & 249 & $\begin{array}{r}180143 \\
(96.36 \%)\end{array}$ & $\begin{array}{r}78 \\
(0.04 \%)\end{array}$ & 121 & 11 & $\begin{array}{r}120 \\
(0.06 \%)\end{array}$ & $\begin{array}{r}95 \\
(79.17 \%)\end{array}$ & $\begin{array}{r}18 \\
(15.00 \%)\end{array}$ & $\begin{array}{r}0 \\
(0.00 \%)\end{array}$ & $\begin{array}{r}7 \\
(5.83 \%)\end{array}$ \\
\hline 2 & 180226 & $\begin{array}{r}0 \\
(0.00 \%)\end{array}$ & $\begin{array}{r}41560 \\
(23.06 \%)\end{array}$ & 97219 & $2 \rightarrow 3$ & 5078 & $\begin{array}{r}180026 \\
(99.89 \%)\end{array}$ & $\begin{array}{r}59 \\
(0.03 \%)\end{array}$ & 303 & 883 & $\begin{array}{r}199 \\
(0.11 \%)\end{array}$ & $\begin{array}{r}143 \\
(71.86 \%)\end{array}$ & $\begin{array}{r}49 \\
(24.62 \%)\end{array}$ & $\begin{array}{r}0 \\
(0.00 \%)\end{array}$ & $\begin{array}{r}7 \\
(3.52 \%)\end{array}$ \\
\hline 3 & 181858 & $\begin{array}{r}0 \\
(0.00 \%)\end{array}$ & $\begin{array}{r}42160 \\
(23.18 \%)\end{array}$ & 98047 & $3 \rightarrow 4$ & 3060 & $\begin{array}{r}181400 \\
(99.75 \%)\end{array}$ & $\begin{array}{r}208 \\
(0.11 \%)\end{array}$ & 291 & 308 & $\begin{array}{r}447 \\
(0.25 \%)\end{array}$ & $\begin{array}{r}338 \\
(75.62 \%)\end{array}$ & $\begin{array}{r}88 \\
(19.69 \%)\end{array}$ & $\begin{array}{r}0 \\
(0.00 \%)\end{array}$ & $\begin{array}{r}21 \\
(4.70 \%)\end{array}$ \\
\hline 4 & 182288 & $\begin{array}{r}0 \\
(0.00 \%)\end{array}$ & $\begin{array}{r}42160 \\
(23.13 \%)\end{array}$ & 98231 & $4 \rightarrow 5$ & 6365 & $\begin{array}{r}179810 \\
(98.64 \%)\end{array}$ & $\begin{array}{r}1174 \\
(0.64 \%)\end{array}$ & 943 & 963 & $\begin{array}{r}1653 \\
(0.91 \%)\end{array}$ & $\begin{array}{r}516 \\
(31.22 \%)\end{array}$ & $\begin{array}{r}988 \\
(59.77 \%)\end{array}$ & $\begin{array}{r}0 \\
(0.00 \%)\end{array}$ & $\begin{array}{r}149 \\
(9.01 \%)\end{array}$ \\
\hline 5 & 181241 & $\begin{array}{r}0 \\
(0.00 \%)\end{array}$ & $\begin{array}{r}41808 \\
(23.07 \%)\end{array}$ & 97627 & $5 \rightarrow 6$ & 605 & $\begin{array}{r}181126 \\
(99.94 \%)\end{array}$ & $\begin{array}{r}24 \\
(0.01 \%)\end{array}$ & 198 & 66 & $\begin{array}{r}114 \\
(0.06 \%)\end{array}$ & $\begin{array}{r}89 \\
(78.07 \%)\end{array}$ & $\begin{array}{r}18 \\
(15.79 \%)\end{array}$ & $\begin{array}{r}0 \\
(0.00 \%)\end{array}$ & $\begin{array}{r}7 \\
(6.14 \%)\end{array}$ \\
\hline 6 & 181366 & $\begin{array}{r}0 \\
(0.00 \%)\end{array}$ & $\begin{array}{r}41853 \\
(23.08 \%)\end{array}$ & 97683 & $6 \rightarrow 7$ & 1855 & $\begin{array}{r}180898 \\
(99.74 \%)\end{array}$ & $\begin{array}{r}212 \\
(0.12 \%)\end{array}$ & 538 & 124 & $\begin{array}{r}467 \\
(0.26 \%)\end{array}$ & $\begin{array}{r}203 \\
(43.47 \%)\end{array}$ & $\begin{array}{r}255 \\
(54.60 \%)\end{array}$ & $\begin{array}{r}0 \\
(0.00 \%)\end{array}$ & $\begin{array}{r}9 \\
(1.93 \%)\end{array}$ \\
\hline 7 & 181437 & $\begin{array}{r}0 \\
(0.00 \%)\end{array}$ & $\begin{array}{r}41867 \\
(23.08 \%)\end{array}$ & 97721 & $7 \rightarrow 8$ & 2865 & $\begin{array}{r}180846 \\
(99.67 \%)\end{array}$ & $\begin{array}{r}320 \\
(0.18 \%)\end{array}$ & 724 & 308 & $\begin{array}{r}590 \\
(0.33 \%)\end{array}$ & $\begin{array}{r}230 \\
(38.98 \%)\end{array}$ & $\begin{array}{r}323 \\
(54.75 \%)\end{array}$ & $\begin{array}{r}0 \\
(0.00 \%)\end{array}$ & $\begin{array}{r}37 \\
(6.27 \%)\end{array}$ \\
\hline 8 & 181783 & $\begin{array}{r}0 \\
(0.00 \%)\end{array}$ & $\begin{array}{r}41902 \\
(23.05 \%)\end{array}$ & 97935 & $8 \rightarrow 9$ & 1773 & $\begin{array}{r}181355 \\
(99.76 \%)\end{array}$ & $\begin{array}{r}181 \\
(0.10 \%)\end{array}$ & 519 & 103 & $\begin{array}{r}427 \\
(0.23 \%)\end{array}$ & $\begin{array}{r}204 \\
(47.78 \%)\end{array}$ & $\begin{array}{r}214 \\
(50.12 \%)\end{array}$ & $\begin{array}{r}0 \\
(0.00 \%)\end{array}$ & $\begin{array}{r}9 \\
(2.11 \%)\end{array}$ \\
\hline 9 & 181824 & $\begin{array}{r}0 \\
(0.00 \%)\end{array}$ & $\begin{array}{r}41909 \\
(23.05 \%)\end{array}$ & 97956 & $9 \rightarrow 10$ & 2387 & $\begin{array}{r}181361 \\
(99.75 \%)\end{array}$ & $\begin{array}{r}236 \\
(0.13 \%)\end{array}$ & 474 & 318 & $\begin{array}{r}456 \\
(0.25 \%)\end{array}$ & $\begin{array}{r}179 \\
(39.25 \%)\end{array}$ & $\begin{array}{r}270 \\
(59.21 \%)\end{array}$ & $\begin{array}{r}0 \\
(0.00 \%)\end{array}$ & $\begin{array}{r}7 \\
(1.54 \%)\end{array}$ \\
\hline 10 & 182132 & $\begin{array}{r}0 \\
(0.00 \%)\end{array}$ & $\begin{array}{r}41944 \\
(23.03 \%)\end{array}$ & 98172 & & & & & & & & & & & \\
\hline
\end{tabular}


Incremental parsing measurements for WebDSL source YellowGrass.

\begin{tabular}{|c|c|c|c|c|c|c|c|c|c|c|c|c|c|c|c|}
\hline \multirow[b]{2}{*}{ Version } & \multicolumn{3}{|c|}{ Parse Nodes } & \multirow{2}{*}{$\begin{array}{c}\text { Character } \\
\text { Nodes } \\
\text { Count }\end{array}$} & \multirow[b]{2}{*}{ Version } & \multicolumn{3}{|c|}{ Parse Nodes } & \multicolumn{2}{|c|}{ Shift } & \multicolumn{5}{|c|}{ Breakdown } \\
\hline & Count & $\begin{array}{l}\text { Ambi- } \\
\text { guous }\end{array}$ & $\begin{array}{c}\text { Irre- } \\
\text { usable }\end{array}$ & & & Created & Reused & Rebuilt & $\begin{array}{l}\text { Parse } \\
\text { Node }\end{array}$ & $\begin{array}{c}\text { Character } \\
\text { Node }\end{array}$ & Count & $\begin{array}{l}\text { Contains } \\
\text { Change }\end{array}$ & $\begin{array}{l}\text { Irre- } \\
\text { usable }\end{array}$ & $\begin{array}{c}\text { No } \\
\text { Actions }\end{array}$ & $\begin{array}{l}\text { Wrong } \\
\text { State }\end{array}$ \\
\hline $\begin{array}{c}\text { Average } \\
(0 . .14)\end{array}$ & 332492 & $\begin{array}{r}0 \\
(0.00 \%)\end{array}$ & $\begin{array}{r}65441 \\
(19.68 \%)\end{array}$ & 166333 & $\begin{array}{c}\text { Average } \\
(1 . .15)\end{array}$ & 6632 & $\begin{array}{r}331816 \\
(99.79 \%)\end{array}$ & $\begin{array}{r}301 \\
(0.09 \%)\end{array}$ & 334 & 1220 & $\begin{array}{r}549 \\
(0.17 \%)\end{array}$ & $\begin{array}{r}237 \\
(49.13 \%)\end{array}$ & $\begin{array}{r}286 \\
(46.03 \%)\end{array}$ & $\begin{array}{r}0 \\
(0.00 \%)\end{array}$ & $\begin{array}{r}26 \\
(4.84 \%)\end{array}$ \\
\hline & & & & & $\rightarrow 0$ & 703238 & & & 0 & 159026 & & & & & \\
\hline 0 & 318753 & $\begin{array}{r}0 \\
(0.00 \%)\end{array}$ & $\begin{array}{r}63030 \\
(19.77 \%)\end{array}$ & 158975 & $0 \rightarrow 1$ & 11089 & $\begin{array}{r}317608 \\
(99.64 \%)\end{array}$ & $\begin{array}{r}563 \\
(0.18 \%)\end{array}$ & 653 & 2080 & $\begin{array}{r}1045 \\
(0.33 \%)\end{array}$ & $\begin{array}{r}486 \\
(46.51 \%)\end{array}$ & $\begin{array}{r}529 \\
(50.62 \%)\end{array}$ & $\begin{array}{r}0 \\
(0.00 \%)\end{array}$ & $\begin{array}{r}30 \\
(2.87 \%)\end{array}$ \\
\hline 1 & 321421 & $\begin{array}{r}0 \\
(0.00 \%)\end{array}$ & $\begin{array}{r}63523 \\
(19.76 \%)\end{array}$ & 160568 & $1 \rightarrow 2$ & 9470 & $\begin{array}{r}317565 \\
(98.80 \%)\end{array}$ & $\begin{array}{r}1344 \\
(0.42 \%)\end{array}$ & 970 & 1014 & $\begin{array}{r}2193 \\
(0.68 \%)\end{array}$ & $\begin{array}{r}790 \\
(36.02 \%)\end{array}$ & $\begin{array}{r}1245 \\
(56.77 \%)\end{array}$ & $\begin{array}{r}0 \\
(0.00 \%)\end{array}$ & $\begin{array}{r}158 \\
(7.20 \%)\end{array}$ \\
\hline 2 & 320082 & $\begin{array}{r}0 \\
(0.00 \%)\end{array}$ & $\begin{array}{r}63043 \\
(19.70 \%)\end{array}$ & 159970 & $2 \rightarrow 3$ & 4400 & $\begin{array}{r}319478 \\
(99.81 \%)\end{array}$ & $\begin{array}{r}306 \\
(0.10 \%)\end{array}$ & 388 & 670 & $\begin{array}{r}576 \\
(0.18 \%)\end{array}$ & $\begin{array}{r}246 \\
(42.71 \%)\end{array}$ & $\begin{array}{r}290 \\
(50.35 \%)\end{array}$ & $\begin{array}{r}0 \\
(0.00 \%)\end{array}$ & $\begin{array}{r}40 \\
(6.94 \%)\end{array}$ \\
\hline 3 & 321187 & $\begin{array}{r}0 \\
(0.00 \%)\end{array}$ & $\begin{array}{r}63228 \\
(19.69 \%)\end{array}$ & 160445 & $3 \rightarrow 4$ & 13573 & $\begin{array}{r}319903 \\
(99.60 \%)\end{array}$ & $\begin{array}{r}791 \\
(0.25 \%)\end{array}$ & 818 & 1714 & $\begin{array}{r}1297 \\
(0.40 \%)\end{array}$ & $\begin{array}{r}411 \\
(31.69 \%)\end{array}$ & $\begin{array}{r}853 \\
(65.77 \%)\end{array}$ & $\begin{array}{r}0 \\
(0.00 \%)\end{array}$ & $\begin{array}{r}33 \\
(2.54 \%)\end{array}$ \\
\hline 4 & 323904 & $\begin{array}{r}0 \\
(0.00 \%)\end{array}$ & $\begin{array}{r}64046 \\
(19.77 \%)\end{array}$ & 161720 & $4 \rightarrow 5$ & 936 & $\begin{array}{r}323678 \\
(99.93 \%)\end{array}$ & $\begin{array}{r}189 \\
(0.06 \%)\end{array}$ & 176 & 99 & $\begin{array}{r}229 \\
(0.07 \%)\end{array}$ & $\begin{array}{r}65 \\
(28.38 \%)\end{array}$ & $\begin{array}{r}126 \\
(55.02 \%)\end{array}$ & $\begin{array}{r}0 \\
(0.00 \%)\end{array}$ & $\begin{array}{r}38 \\
(16.59 \%)\end{array}$ \\
\hline 5 & 323900 & $\begin{array}{r}0 \\
(0.00 \%)\end{array}$ & $\begin{array}{r}64047 \\
(19.77 \%)\end{array}$ & 161716 & $5 \rightarrow 6$ & 6626 & $\begin{array}{r}323741 \\
(99.95 \%)\end{array}$ & $\begin{array}{r}81 \\
(0.03 \%)\end{array}$ & 157 & 1679 & $\begin{array}{r}157 \\
(0.05 \%)\end{array}$ & $\begin{array}{r}42 \\
(26.75 \%)\end{array}$ & $\begin{array}{r}96 \\
(61.15 \%)\end{array}$ & $\begin{array}{r}0 \\
(0.00 \%)\end{array}$ & $\begin{array}{r}19 \\
(12.10 \%)\end{array}$ \\
\hline 6 & 326852 & $\begin{array}{r}0 \\
(0.00 \%)\end{array}$ & $\begin{array}{r}64504 \\
(19.73 \%)\end{array}$ & 163359 & $6 \rightarrow 7$ & 345 & $\begin{array}{r}326756 \\
(99.97 \%)\end{array}$ & $\begin{array}{r}26 \\
(0.01 \%)\end{array}$ & 105 & 49 & $\begin{array}{r}96 \\
(0.03 \%)\end{array}$ & $\begin{array}{r}63 \\
(65.63 \%)\end{array}$ & $\begin{array}{r}33 \\
(34.38 \%)\end{array}$ & $\begin{array}{r}0 \\
(0.00 \%)\end{array}$ & $\begin{array}{r}0 \\
(0.00 \%)\end{array}$ \\
\hline 7 & 326874 & $\begin{array}{r}0 \\
(0.00 \%)\end{array}$ & $\begin{array}{r}64504 \\
(19.73 \%)\end{array}$ & 163370 & $7 \rightarrow 8$ & 21228 & $\begin{array}{r}326775 \\
(99.97 \%)\end{array}$ & $\begin{array}{r}72 \\
(0.02 \%)\end{array}$ & 148 & 5297 & $\begin{array}{r}98 \\
(0.03 \%)\end{array}$ & $\begin{array}{r}22 \\
(22.45 \%)\end{array}$ & $\begin{array}{r}72 \\
(73.47 \%)\end{array}$ & $\begin{array}{r}0 \\
(0.00 \%)\end{array}$ & $\begin{array}{r}4 \\
(4.08 \%)\end{array}$ \\
\hline 8 & 335813 & $\begin{array}{r}0 \\
(000 \%)\end{array}$ & $\begin{array}{r}65837 \\
(1961 \%)\end{array}$ & 168635 & $8 \rightarrow 9$ & 19230 & & & 53 & 4062 & & & & & \\
\hline 9 & 344778 & $\begin{array}{r}(0.00 \%) \\
0\end{array}$ & $\begin{array}{r}(19.61 \%) \\
67624 \\
\end{array}$ & 172696 & $9 \rightarrow 10$ & 977 & $\begin{array}{r}344565 \\
(99.94 \%)\end{array}$ & $\begin{array}{r}89 \\
(0.03 \%)\end{array}$ & 93 & 178 & $\begin{array}{r}183 \\
(0.05 \%)\end{array}$ & $\begin{array}{r}135 \\
(73.77 \%)\end{array}$ & $\begin{array}{r}32 \\
(17.49 \%)\end{array}$ & $\begin{array}{r}0 \\
(0.00 \%)\end{array}$ & $\begin{array}{r}16 \\
(8.74 \%)\end{array}$ \\
\hline & & $\begin{array}{r}(0.00 \%) \\
0\end{array}$ & $\begin{array}{r}(19.61 \%) \\
67645\end{array}$ & $17-00$ & $10 \rightarrow 11$ & 481 & $\begin{array}{r}344782 \\
(99.96 \%)\end{array}$ & $\begin{array}{r}32 \\
(0.01 \%)\end{array}$ & 121 & 102 & $\begin{array}{r}150 \\
(0.04 \%)\end{array}$ & $\begin{array}{r}120 \\
(80.00 \%)\end{array}$ & $\begin{array}{r}30 \\
(20.00 \%)\end{array}$ & $\begin{array}{r}0 \\
(0.00 \%)\end{array}$ & $\begin{array}{r}0 \\
(0.00 \%)\end{array}$ \\
\hline 10 & 344930 & $\begin{array}{r}(0.00 \%) \\
0\end{array}$ & $\begin{array}{r}(19.61 \%) \\
67645\end{array}$ & $1 / 2 / 9$ & $11 \rightarrow 12$ & 144 & $\begin{array}{r}344967 \\
(9999 \%)\end{array}$ & $\begin{array}{r}19 \\
(0.01 \%)\end{array}$ & 91 & 16 & $\begin{array}{r}45 \\
(001 \%)\end{array}$ & $\begin{array}{r}21 \\
(4667 \%)\end{array}$ & $\begin{array}{r}24 \\
(5333 \%\end{array}$ & $\begin{array}{r}0 \\
(000 \%)\end{array}$ & 0 \\
\hline 11 & 345013 & $(0.00 \%)$ & $(19.61 \%)$ & 172833 & 13 & 844 & 344215 & $\begin{array}{r}(0.0110) \\
157\end{array}$ & 170 & 70 & $\begin{array}{r}(0.01 \%) \\
799\end{array}$ & $\begin{array}{r}(46.6 / \%) \\
603\end{array}$ & $\begin{array}{r}(53.33 \%) \\
195\end{array}$ & $\begin{array}{r}(0.00 \%) \\
0\end{array}$ & $\begin{array}{r}(0.00 \%) \\
1\end{array}$ \\
\hline 12 & 345015 & $\begin{array}{r}0 \\
(0.00 \%)\end{array}$ & $\begin{array}{r}67645 \\
(1961 \%)\end{array}$ & 172834 & & 044 & $\begin{array}{l}(99.77 \%) \\
\end{array}$ & $\begin{array}{r}(0.05 \%) \\
41\end{array}$ & 170 & 10 & $(0.23 \%)$ & $\begin{array}{r}(75.47 \%) \\
\end{array}$ & $(24.41 \%)$ & $(0.00 \%)$ & $(0.13 \%)$ \\
\hline 13 & 344461 & $\begin{array}{r}(0.00 \%) \\
0\end{array}$ & $\begin{array}{r}(19.61 \%) \\
67645\end{array}$ & 557 & $13 \rightarrow 14$ & 420 & $\begin{array}{r}344235 \\
(99.93 \%)\end{array}$ & $\begin{array}{r}41 \\
(0.01 \%)\end{array}$ & 121 & 72 & $\begin{array}{r}142 \\
(0.04 \%)\end{array}$ & $\begin{array}{r}108 \\
(76.06 \%)\end{array}$ & $\begin{array}{r}30 \\
(21.13 \%)\end{array}$ & $(0.00 \%)$ & $\begin{array}{r}4 \\
(2.82 \%)\end{array}$ \\
\hline 14 & 344393 & $\begin{array}{r}(0.00 \%) \\
0 \\
(0.00 \%)\end{array}$ & $\begin{array}{r}(19.64 \%) \\
67645 \\
(19.64 \%)\end{array}$ & 172529 & $14 \rightarrow 15$ & 9710 & $\begin{array}{r}343164 \\
(99.64 \%)\end{array}$ & $\begin{array}{r}810 \\
(0.24 \%)\end{array}$ & 947 & 1202 & $\begin{array}{r}1222 \\
(0.35 \%)\end{array}$ & $\begin{array}{r}437 \\
(35.76 \%)\end{array}$ & $\begin{array}{r}740 \\
(60.56 \%)\end{array}$ & $\begin{array}{r}0 \\
(0.00 \%)\end{array}$ & $\begin{array}{r}45 \\
(3.68 \%)\end{array}$ \\
\hline 15 & 346034 & $\begin{array}{r}0 \\
(0.00 \%)\end{array}$ & $\begin{array}{r}67996 \\
(19.65 \%)\end{array}$ & 173274 & & & & & & & & & & & \\
\hline
\end{tabular}


Incremental parsing measurements for WebDSL source elib-utils.

\begin{tabular}{|c|c|c|c|c|c|c|c|c|c|c|c|c|c|c|c|}
\hline \multirow[b]{2}{*}{ Version } & \multicolumn{3}{|c|}{ Parse Nodes } & \multirow{2}{*}{$\begin{array}{c}\text { Character } \\
\text { Nodes } \\
\text { Count }\end{array}$} & \multirow[b]{2}{*}{ Version } & \multicolumn{3}{|c|}{ Parse Nodes } & \multicolumn{2}{|c|}{ Shift } & \multicolumn{5}{|c|}{ Breakdown } \\
\hline & Count & $\begin{array}{l}\text { Ambi- } \\
\text { guous }\end{array}$ & $\begin{array}{c}\text { Irre- } \\
\text { usable }\end{array}$ & & & Created & Reused & Rebuilt & $\begin{array}{l}\text { Parse } \\
\text { Node }\end{array}$ & $\begin{array}{l}\text { Character } \\
\text { Node }\end{array}$ & Count & $\begin{array}{l}\text { Contains } \\
\text { Change }\end{array}$ & $\begin{array}{c}\text { Irre- } \\
\text { usable }\end{array}$ & $\begin{array}{c}\text { No } \\
\text { Actions }\end{array}$ & $\begin{array}{l}\text { Wrong } \\
\text { State }\end{array}$ \\
\hline $\begin{array}{c}\text { Average } \\
(0 . .14)\end{array}$ & 80827 & $\begin{array}{r}0 \\
(0.00 \%)\end{array}$ & $\begin{array}{r}17136 \\
(21.20 \%)\end{array}$ & 39406 & $\begin{array}{c}\text { Average } \\
(1 . .15)\end{array}$ & 1874 & $\begin{array}{r}80481 \\
(99.57 \%) \\
\end{array}$ & $\begin{array}{r}172 \\
(0.21 \%)\end{array}$ & 181 & 204 & $\begin{array}{r}326 \\
(0.40 \%) \\
\end{array}$ & $\begin{array}{r}148 \\
(48.75 \%) \\
\end{array}$ & $\begin{array}{r}160 \\
(45.97 \%)\end{array}$ & $\begin{array}{r}0 \\
(0.00 \%)\end{array}$ & $\begin{array}{r}18 \\
(5.28 \%) \\
\end{array}$ \\
\hline & & & & & $\rightarrow 0$ & 187245 & & & 0 & 38972 & & & & & \\
\hline 0 & 80035 & $\begin{array}{r}0 \\
(0.00 \%)\end{array}$ & $\begin{array}{r}16982 \\
(21.22 \%)\end{array}$ & 38955 & $0 \rightarrow 1$ & 327 & $\begin{array}{r}79962 \\
(99.91 \%)\end{array}$ & $\begin{array}{r}65 \\
(0.08 \%)\end{array}$ & 73 & 22 & $\begin{array}{r}74 \\
(0.09 \%)\end{array}$ & $\begin{array}{r}41 \\
(55.41 \%)\end{array}$ & $\begin{array}{r}33 \\
(44.59 \%)\end{array}$ & $\begin{array}{r}0 \\
(0.00 \%)\end{array}$ & $\begin{array}{r}0 \\
(0.00 \%)\end{array}$ \\
\hline 1 & 80033 & $\begin{array}{r}0 \\
(0.00 \%)\end{array}$ & $\begin{array}{r}16982 \\
(21.22 \%)\end{array}$ & 38953 & $1 \rightarrow 2$ & 3529 & $\begin{array}{r}79550 \\
(99.40 \%)\end{array}$ & $\begin{array}{r}381 \\
(0.48 \%)\end{array}$ & 170 & 375 & $\begin{array}{r}482 \\
(0.60 \%)\end{array}$ & $\begin{array}{r}31 \\
(6.43 \%)\end{array}$ & $\begin{array}{r}449 \\
(93.15 \%)\end{array}$ & $\begin{array}{r}0 \\
(0.00 \%)\end{array}$ & $\begin{array}{r}2 \\
(0.41 \%)\end{array}$ \\
\hline 2 & 80255 & $\begin{array}{r}0 \\
(0.00 \%)\end{array}$ & $\begin{array}{r}16986 \\
(21.17 \%)\end{array}$ & 39118 & $2 \rightarrow 3$ & 3138 & $\begin{array}{r}79671 \\
(99.27 \%)\end{array}$ & $\begin{array}{r}328 \\
(0.41 \%)\end{array}$ & 227 & 323 & $\begin{array}{r}523 \\
(0.65 \%)\end{array}$ & $\begin{array}{r}165 \\
(31.55 \%)\end{array}$ & $\begin{array}{r}297 \\
(56.79 \%)\end{array}$ & $\begin{array}{r}0 \\
(0.00 \%)\end{array}$ & $\begin{array}{r}61 \\
(11.66 \%)\end{array}$ \\
\hline 3 & 80437 & $\begin{array}{r}0 \\
(0.00 \%)\end{array}$ & $\begin{array}{r}17030 \\
(21.17 \%)\end{array}$ & 39211 & $3 \rightarrow 4$ & 1085 & $\begin{array}{r}80424 \\
(99.98 \%)\end{array}$ & $\begin{array}{r}7 \\
(0.01 \%)\end{array}$ & 31 & 169 & $\begin{array}{r}12 \\
(0.01 \%)\end{array}$ & $\begin{array}{r}7 \\
(58.33 \%)\end{array}$ & $\begin{array}{r}5 \\
(41.67 \%)\end{array}$ & $\begin{array}{r}0 \\
(0.00 \%)\end{array}$ & $(0.00 \%)$ \\
\hline 4 & 80765 & $\begin{array}{r}0 \\
(0.00 \%)\end{array}$ & $\begin{array}{r}17147 \\
(21.23 \%)\end{array}$ & 39378 & $4 \rightarrow 5$ & 1125 & $\begin{array}{r}80417 \\
(99.57 \%)\end{array}$ & $\begin{array}{r}109 \\
(0.13 \%)\end{array}$ & 111 & 104 & $\begin{array}{r}308 \\
(0.38 \%)\end{array}$ & $\begin{array}{r}215 \\
(69.81 \%)\end{array}$ & $\begin{array}{r}86 \\
(27.92 \%)\end{array}$ & $\begin{array}{r}0 \\
(0.00 \%)\end{array}$ & $\begin{array}{r}7 \\
(2.27 \%)\end{array}$ \\
\hline 5 & 80669 & $\begin{array}{r}0 \\
(0.00 \%)\end{array}$ & $\begin{array}{r}17106 \\
(21.21 \%)\end{array}$ & 39317 & $5 \rightarrow 6$ & 1378 & $\begin{array}{r}80053 \\
(99.24 \%)\end{array}$ & $\begin{array}{r}376 \\
(0.47 \%)\end{array}$ & 296 & 71 & $\begin{array}{r}661 \\
(0.82 \%)\end{array}$ & $\begin{array}{r}512 \\
(77.46 \%)\end{array}$ & $\begin{array}{r}101 \\
(15.28 \%)\end{array}$ & $\begin{array}{r}0 \\
(0.00 \%)\end{array}$ & $\begin{array}{r}48 \\
(7.26 \%)\end{array}$ \\
\hline 6 & 80575 & $\begin{array}{r}0 \\
(0.00 \%)\end{array}$ & $\begin{array}{r}17112 \\
(21.24 \%)\end{array}$ & 39283 & $6 \rightarrow 7$ & 628 & $\begin{array}{r}80498 \\
(99.90 \%)\end{array}$ & $\begin{array}{r}45 \\
(0.06 \%)\end{array}$ & 71 & 57 & $\begin{array}{r}74 \\
(0.09 \%)\end{array}$ & $\begin{array}{r}38 \\
(51.35 \%)\end{array}$ & $\begin{array}{r}36 \\
(48.65 \%)\end{array}$ & $\begin{array}{r}0 \\
(0.00 \%)\end{array}$ & $\begin{array}{r}0 \\
(0.00 \%)\end{array}$ \\
\hline 7 & 80645 & $\begin{array}{r}0 \\
(0.00 \%)\end{array}$ & $\begin{array}{r}17140 \\
(21.25 \%)\end{array}$ & 39320 & $7 \rightarrow 8$ & 655 & $\begin{array}{r}80368 \\
(99.66 \%)\end{array}$ & $\begin{array}{r}99 \\
(0.12 \%)\end{array}$ & 133 & 84 & $\begin{array}{r}240 \\
(0.30 \%)\end{array}$ & $\begin{array}{r}45 \\
(18.75 \%)\end{array}$ & $\begin{array}{r}123 \\
(51.25 \%)\end{array}$ & $\begin{array}{r}0 \\
(0.00 \%)\end{array}$ & $\begin{array}{r}72 \\
(30.00 \%)\end{array}$ \\
\hline 8 & 80553 & $\begin{array}{r}0 \\
(0.00 \%)\end{array}$ & $\begin{array}{r}17093 \\
(21.22 \%)\end{array}$ & 39302 & $8 \rightarrow 9$ & 893 & $\begin{array}{r}80537 \\
(99.98 \%)\end{array}$ & $\begin{array}{r}9 \\
(0.01 \%)\end{array}$ & 34 & 131 & $\begin{array}{r}15 \\
(0.02 \%)\end{array}$ & $\begin{array}{r}7 \\
(46.67 \%)\end{array}$ & $\begin{array}{r}8 \\
(53.33 \%)\end{array}$ & $\begin{array}{r}0 \\
(0.00 \%)\end{array}$ & $(0.00 \%)$ \\
\hline 9 & 80843 & $\begin{array}{r}0 \\
(0.00 \%)\end{array}$ & $\begin{array}{r}17182 \\
(21.25 \%)\end{array}$ & 39430 & $9 \rightarrow 10$ & 1425 & $\begin{array}{r}80517 \\
(99.60 \%)\end{array}$ & $\begin{array}{r}150 \\
(0.19 \%)\end{array}$ & 193 & 166 & $\begin{array}{r}314 \\
(0.39 \%)\end{array}$ & $\begin{array}{r}122 \\
(38.85 \%)\end{array}$ & $\begin{array}{r}145 \\
(46.18 \%)\end{array}$ & $\begin{array}{r}0 \\
(0.00 \%)\end{array}$ & $\begin{array}{r}47 \\
(14.97 \%)\end{array}$ \\
\hline 10 & 80975 & $\begin{array}{r}0 \\
(0.00 \%)\end{array}$ & $\begin{array}{r}17182 \\
(21.22 \%)\end{array}$ & 39496 & $10 \rightarrow 11$ & 1260 & $\begin{array}{r}80610 \\
(99.55 \%)\end{array}$ & $\begin{array}{r}237 \\
(0.29 \%)\end{array}$ & 195 & 82 & $\begin{array}{r}354 \\
(0.44 \%)\end{array}$ & $\begin{array}{r}100 \\
(28.25 \%)\end{array}$ & $\begin{array}{r}236 \\
(66.67 \%)\end{array}$ & $\begin{array}{r}0 \\
(0.00 \%)\end{array}$ & $\begin{array}{r}18 \\
(5.08 \%)\end{array}$ \\
\hline 11 & 80921 & $\begin{array}{r}0 \\
(0.00 \%)\end{array}$ & $\begin{array}{r}17160 \\
(21.21 \%)\end{array}$ & 39480 & $11 \rightarrow 12$ & 1040 & $\begin{array}{r}80617 \\
(99.62 \%)\end{array}$ & $\begin{array}{r}106 \\
(0.13 \%)\end{array}$ & 267 & 124 & $\begin{array}{r}303 \\
(0.37 \%)\end{array}$ & $\begin{array}{r}183 \\
(60.40 \%)\end{array}$ & $\begin{array}{r}100 \\
(33.00 \%)\end{array}$ & $\begin{array}{r}0 \\
(0.00 \%)\end{array}$ & $\begin{array}{r}20 \\
(6.60 \%)\end{array}$ \\
\hline 12 & 81043 & $\begin{array}{r}0 \\
(0.00 \%)\end{array}$ & $\begin{array}{r}17160 \\
(21.17 \%)\end{array}$ & 39547 & $12 \rightarrow 13$ & 1425 & $\begin{array}{r}81008 \\
(99.96 \%)\end{array}$ & $\begin{array}{r}9 \\
(0.01 \%)\end{array}$ & 60 & 278 & $\begin{array}{r}34 \\
(0.04 \%)\end{array}$ & $\begin{array}{r}26 \\
(76.47 \%)\end{array}$ & $\begin{array}{r}8 \\
(23.53 \%)\end{array}$ & $\begin{array}{r}0 \\
(0.00 \%)\end{array}$ & $\begin{array}{r}0 \\
(0.00 \%)\end{array}$ \\
\hline 13 & 81539 & $\begin{array}{r}0 \\
(0.00 \%)\end{array}$ & $\begin{array}{r}17274 \\
(21.18 \%)\end{array}$ & 39821 & $13 \rightarrow 14$ & 8686 & $\begin{array}{r}80084 \\
(98.22 \%)\end{array}$ & $\begin{array}{r}569 \\
(0.70 \%)\end{array}$ & 742 & 900 & $\begin{array}{r}1285 \\
(1.58 \%)\end{array}$ & $\begin{array}{r}587 \\
(45.68 \%)\end{array}$ & $\begin{array}{r}698 \\
(54.32 \%)\end{array}$ & $\begin{array}{r}0 \\
(0.00 \%)\end{array}$ & $\begin{array}{r}0 \\
(0.00 \%)\end{array}$ \\
\hline 14 & 83116 & $\begin{array}{r}0 \\
(0.00 \%)\end{array}$ & $\begin{array}{r}17504 \\
(21.06 \%)\end{array}$ & 40480 & $14 \rightarrow 15$ & 1518 & $\begin{array}{r}82900 \\
(99.74 \%)\end{array}$ & $\begin{array}{r}84 \\
(0.10 \%)\end{array}$ & 108 & 168 & $\begin{array}{r}217 \\
(0.26 \%)\end{array}$ & $\begin{array}{r}143 \\
(65.90 \%)\end{array}$ & $\begin{array}{r}72 \\
(33.18 \%)\end{array}$ & $\begin{array}{r}0 \\
(0.00 \%)\end{array}$ & $\begin{array}{r}2 \\
(0.92 \%)\end{array}$ \\
\hline 15 & 83373 & $\begin{array}{r}0 \\
(0.00 \%)\end{array}$ & $\begin{array}{r}17593 \\
(21.10 \%)\end{array}$ & 40589 & & & & & & & & & & & \\
\hline
\end{tabular}




\section{$\stackrel{\text { A }}{\circ} \quad$ A.1.3 SDF3}

Incremental parsing measurements for the SDF3 language.

\begin{tabular}{|c|c|c|c|c|c|c|c|c|c|c|c|c|c|c|c|}
\hline \multirow[b]{2}{*}{ Source } & \multicolumn{3}{|c|}{ Parse Nodes } & \multirow{2}{*}{$\begin{array}{c}\text { Character } \\
\text { Nodes } \\
\text { Count }\end{array}$} & \multirow[b]{2}{*}{ Source } & \multicolumn{3}{|c|}{ Parse Nodes } & \multicolumn{2}{|c|}{ Shift } & \multicolumn{5}{|c|}{ Breakdown } \\
\hline & Count & $\begin{array}{l}\text { Ambi- } \\
\text { guous }\end{array}$ & $\begin{array}{c}\text { Irre- } \\
\text { usable }\end{array}$ & & & Created & Reused & Rebuilt & $\begin{array}{l}\text { Parse } \\
\text { Node }\end{array}$ & $\begin{array}{c}\text { Character } \\
\text { Node }\end{array}$ & Count & $\begin{array}{l}\text { Contains } \\
\text { Change }\end{array}$ & $\begin{array}{c}\text { Irre- } \\
\text { usable }\end{array}$ & $\begin{array}{c}\text { No } \\
\text { Actions }\end{array}$ & $\begin{array}{c}\text { Wrong } \\
\text { State }\end{array}$ \\
\hline Average & 110664 & $1.01 \%$ & $41.36 \%$ & 57095 & Average & 5725 & $98.21 \%$ & $1.21 \%$ & 279 & 778 & $1.57 \%$ & $19.35 \%$ & $78.28 \%$ & $0.00 \%$ & $2.38 \%$ \\
\hline $\mathrm{NaBL}$ & 194597 & $0.88 \%$ & $39.72 \%$ & 98595 & $\mathrm{NaBL}$ & 7174 & $99.40 \%$ & $0.37 \%$ & 374 & 912 & $0.44 \%$ & $18.74 \%$ & $79.14 \%$ & $0.00 \%$ & $2.12 \%$ \\
\hline DynSem & 20549 & $1.23 \%$ & $40.08 \%$ & 9680 & DynSem & 2200 & $98.15 \%$ & $1.25 \%$ & 132 & 300 & $1.72 \%$ & $20.64 \%$ & $78.00 \%$ & $0.00 \%$ & $1.36 \%$ \\
\hline FlowSpec & 24064 & $1.07 \%$ & $48.77 \%$ & 12079 & FlowSpec & 6187 & $95.14 \%$ & $3.04 \%$ & 254 & 798 & $4.05 \%$ & $21.18 \%$ & $77.06 \%$ & $0.00 \%$ & $1.77 \%$ \\
\hline Stratego & 151528 & $0.72 \%$ & $36.82 \%$ & 79123 & Stratego & 6874 & $99.25 \%$ & $0.62 \%$ & 303 & 864 & $0.73 \%$ & $24.81 \%$ & $69.59 \%$ & $0.00 \%$ & $5.60 \%$ \\
\hline WebDSL & 162581 & $1.14 \%$ & $41.39 \%$ & 85998 & WebDSL & 6191 & $99.10 \%$ & $0.79 \%$ & 334 & 1017 & $0.89 \%$ & $11.37 \%$ & $87.58 \%$ & $0.00 \%$ & $1.05 \%$ \\
\hline
\end{tabular}


Incremental parsing measurements for SDF3 source NaBL.

\begin{tabular}{|c|c|c|c|c|c|c|c|c|c|c|c|c|c|c|c|}
\hline \multirow[b]{2}{*}{ Version } & \multicolumn{3}{|c|}{ Parse Nodes } & \multirow{2}{*}{$\begin{array}{c}\text { Character } \\
\text { Nodes } \\
\text { Count } \\
\end{array}$} & \multirow[b]{2}{*}{ Version } & \multicolumn{3}{|c|}{ Parse Nodes } & \multicolumn{2}{|c|}{ Shift } & \multicolumn{5}{|c|}{ Breakdown } \\
\hline & Count & $\begin{array}{l}\text { Ambi- } \\
\text { guous }\end{array}$ & $\begin{array}{c}\text { Irre- } \\
\text { usable }\end{array}$ & & & Created & Reused & Rebuilt & $\begin{array}{l}\text { Parse } \\
\text { Node }\end{array}$ & $\begin{array}{c}\text { Character } \\
\text { Node }\end{array}$ & Count & $\begin{array}{l}\text { Contains } \\
\text { Change }\end{array}$ & $\begin{array}{c}\text { Irre- } \\
\text { usable }\end{array}$ & $\begin{array}{c}\text { No } \\
\text { Actions }\end{array}$ & $\begin{array}{l}\text { Wrong } \\
\text { State }\end{array}$ \\
\hline $\begin{array}{c}\text { Average } \\
(0 . .14)\end{array}$ & 194597 & $\begin{array}{r}1704 \\
(0.88 \%)\end{array}$ & $\begin{array}{r}77292 \\
(39.72 \%) \\
\end{array}$ & 98595 & $\begin{array}{c}\text { Average } \\
(1 . .15)\end{array}$ & 7174 & $\begin{array}{r}193424 \\
(99.40 \%)\end{array}$ & $\begin{array}{r}731 \\
(0.37 \%)\end{array}$ & 374 & 912 & $\begin{array}{r}866 \\
(0.44 \%)\end{array}$ & $\begin{array}{r}91 \\
(18.74 \%)\end{array}$ & $\begin{array}{r}761 \\
(79.14 \%)\end{array}$ & $\begin{array}{r}0 \\
(0.00 \%)\end{array}$ & $\begin{array}{r}13 \\
(2.12 \%)\end{array}$ \\
\hline & & & & & $\rightarrow 0$ & 512465 & & & 0 & 96664 & & & & & \\
\hline 0 & 190950 & $\begin{array}{r}1675 \\
(0.88 \%)\end{array}$ & $\begin{array}{r}75884 \\
(39.74 \%)\end{array}$ & 96540 & $0 \rightarrow 1$ & 5005 & $\begin{array}{r}190304 \\
(99.66 \%)\end{array}$ & $\begin{array}{r}561 \\
(0.29 \%)\end{array}$ & 278 & 763 & $\begin{array}{r}644 \\
(0.34 \%)\end{array}$ & $\begin{array}{r}49 \\
(7.61 \%)\end{array}$ & $\begin{array}{r}588 \\
(91.30 \%)\end{array}$ & $\begin{array}{r}0 \\
(0.00 \%)\end{array}$ & $\begin{array}{r}7 \\
(1.09 \%)\end{array}$ \\
\hline 1 & 191566 & $\begin{array}{r}1681 \\
(0.88 \%)\end{array}$ & $\begin{array}{r}76083 \\
(39.72 \%)\end{array}$ & 96879 & $1 \rightarrow 2$ & 1335 & $\begin{array}{r}190919 \\
(99.66 \%)\end{array}$ & $\begin{array}{r}262 \\
(0.14 \%)\end{array}$ & 222 & 125 & $\begin{array}{r}374 \\
(0.20 \%)\end{array}$ & $\begin{array}{r}136 \\
(36.36 \%)\end{array}$ & $\begin{array}{r}235 \\
(62.83 \%)\end{array}$ & $\begin{array}{r}0 \\
(0.00 \%)\end{array}$ & $\begin{array}{r}3 \\
(0.80 \%)\end{array}$ \\
\hline 2 & 191211 & $\begin{array}{r}1677 \\
(0.88 \%)\end{array}$ & $\begin{array}{r}75900 \\
(39.69 \%)\end{array}$ & 96705 & $2 \rightarrow 3$ & 933 & $\begin{array}{r}191006 \\
(99.89 \%)\end{array}$ & $\begin{array}{r}156 \\
(0.08 \%)\end{array}$ & 191 & 134 & $\begin{array}{r}207 \\
(0.11 \%)\end{array}$ & $\begin{array}{r}38 \\
(18.36 \%)\end{array}$ & $\begin{array}{r}169 \\
(81.64 \%)\end{array}$ & $\begin{array}{r}0 \\
(0.00 \%)\end{array}$ & $\begin{array}{r}0 \\
(0.00 \%)\end{array}$ \\
\hline 3 & 191212 & $\begin{array}{r}1677 \\
(0.88 \%)\end{array}$ & $\begin{array}{r}75901 \\
(39.69 \%)\end{array}$ & 96706 & $3 \rightarrow 4$ & 11041 & $\begin{array}{r}190313 \\
(99.53 \%)\end{array}$ & $\begin{array}{r}101 \\
(0.05 \%)\end{array}$ & 184 & 2217 & $\begin{array}{r}275 \\
(0.14 \%)\end{array}$ & $\begin{array}{r}165 \\
(60.00 \%)\end{array}$ & $\begin{array}{r}108 \\
(39.27 \%)\end{array}$ & $\begin{array}{r}0 \\
(0.00 \%)\end{array}$ & $\begin{array}{r}2 \\
(0.73 \%)\end{array}$ \\
\hline 4 & 194181 & $\begin{array}{r}1707 \\
(0.88 \%)\end{array}$ & $\begin{array}{r}77100 \\
(39.71 \%)\end{array}$ & 98475 & $4 \rightarrow 5$ & 658 & $\begin{array}{r}194024 \\
(99.92 \%)\end{array}$ & $\begin{array}{r}139 \\
(0.07 \%)\end{array}$ & 179 & 85 & $\begin{array}{r}160 \\
(0.08 \%)\end{array}$ & $\begin{array}{r}37 \\
(23.13 \%)\end{array}$ & $\begin{array}{r}123 \\
(76.88 \%)\end{array}$ & $\begin{array}{r}0 \\
(0.00 \%)\end{array}$ & $\begin{array}{r}0 \\
(0.00 \%)\end{array}$ \\
\hline 5 & 194177 & $\begin{array}{r}1707 \\
(0.88 \%)\end{array}$ & $\begin{array}{r}77096 \\
(39.70 \%)\end{array}$ & 98471 & $5 \rightarrow 6$ & 1492 & $\begin{array}{r}193915 \\
(99.87 \%)\end{array}$ & $\begin{array}{r}245 \\
(0.13 \%)\end{array}$ & 165 & 234 & $\begin{array}{r}261 \\
(0.13 \%)\end{array}$ & $\begin{array}{r}10 \\
(3.83 \%)\end{array}$ & $\begin{array}{r}251 \\
(96.17 \%)\end{array}$ & $\begin{array}{r}0 \\
(0.00 \%)\end{array}$ & $\begin{array}{r}0 \\
(0.00 \%)\end{array}$ \\
\hline 6 & 194255 & $\begin{array}{r}1707 \\
(0.88 \%)\end{array}$ & $\begin{array}{r}77096 \\
(39.69 \%)\end{array}$ & 98540 & $6 \rightarrow 7$ & 13604 & $\begin{array}{r}192231 \\
(98.96 \%)\end{array}$ & $\begin{array}{r}1797 \\
(0.93 \%)\end{array}$ & 741 & 1236 & $\begin{array}{r}2023 \\
(1.04 \%)\end{array}$ & $\begin{array}{r}37 \\
(1.83 \%)\end{array}$ & $\begin{array}{r}1967 \\
(97.23 \%)\end{array}$ & $\begin{array}{r}0 \\
(0.00 \%)\end{array}$ & $\begin{array}{r}19 \\
(0.94 \%)\end{array}$ \\
\hline 7 & 194265 & $\begin{array}{r}1707 \\
(0.88 \%)\end{array}$ & $\begin{array}{r}77099 \\
(39.69 \%)\end{array}$ & 98556 & $7 \rightarrow 8$ & 1832 & $\begin{array}{r}194072 \\
(99.90 \%)\end{array}$ & $\begin{array}{r}145 \\
(0.07 \%)\end{array}$ & 188 & 281 & $\begin{array}{r}192 \\
(0.10 \%)\end{array}$ & $\begin{array}{r}30 \\
(15.63 \%)\end{array}$ & $\begin{array}{r}157 \\
(81.77 \%)\end{array}$ & $\begin{array}{r}0 \\
(0.00 \%)\end{array}$ & $\begin{array}{r}5 \\
(2.60 \%)\end{array}$ \\
\hline 8 & 194617 & $\begin{array}{r}1717 \\
(0.88 \%)\end{array}$ & $\begin{array}{r}77231 \\
(39.68 \%)\end{array}$ & 98731 & $8 \rightarrow 9$ & 1149 & $\begin{array}{r}194411 \\
(99.89 \%)\end{array}$ & $\begin{array}{r}167 \\
(0.09 \%)\end{array}$ & 192 & 120 & $\begin{array}{r}205 \\
(0.11 \%)\end{array}$ & $\begin{array}{r}22 \\
(10.73 \%)\end{array}$ & $\begin{array}{r}173 \\
(84.39 \%)\end{array}$ & $\begin{array}{r}0 \\
(0.00 \%)\end{array}$ & $\begin{array}{r}10 \\
(4.88 \%)\end{array}$ \\
\hline 9 & 194634 & $\begin{array}{r}1717 \\
(0.88 \%)\end{array}$ & $\begin{array}{r}77231 \\
(39.68 \%)\end{array}$ & 98742 & $9 \rightarrow 10$ & 33184 & $\begin{array}{r}188957 \\
(97.08 \%)\end{array}$ & $\begin{array}{r}2682 \\
(1.38 \%)\end{array}$ & 1091 & 4545 & $\begin{array}{r}3105 \\
(1.60 \%)\end{array}$ & $\begin{array}{r}306 \\
(9.86 \%)\end{array}$ & $\begin{array}{r}2760 \\
(88.89 \%)\end{array}$ & $\begin{array}{r}0 \\
(0.00 \%)\end{array}$ & $\begin{array}{r}39 \\
(1.26 \%)\end{array}$ \\
\hline 10 & 197569 & $\begin{array}{r}1717 \\
(0.87 \%)\end{array}$ & $\begin{array}{r}78553 \\
(39.76 \%)\end{array}$ & 100108 & $10 \rightarrow 11$ & 15001 & $\begin{array}{r}195195 \\
(98.80 \%)\end{array}$ & $\begin{array}{r}2002 \\
(1.01 \%)\end{array}$ & 792 & 1452 & $\begin{array}{r}2238 \\
(1.13 \%)\end{array}$ & $\begin{array}{r}71 \\
(3.17 \%)\end{array}$ & $\begin{array}{r}2140 \\
(95.62 \%)\end{array}$ & $\begin{array}{r}0 \\
(0.00 \%)\end{array}$ & $\begin{array}{r}27 \\
(1.21 \%)\end{array}$ \\
\hline 11 & 197573 & $\begin{array}{r}1717 \\
(0.87 \%)\end{array}$ & $\begin{array}{r}78551 \\
(39.76 \%)\end{array}$ & 100110 & $11 \rightarrow 12$ & 15405 & $\begin{array}{r}195000 \\
(98.70 \%)\end{array}$ & $\begin{array}{r}1964 \\
(0.99 \%)\end{array}$ & 789 & 1564 & $\begin{array}{r}2220 \\
(1.12 \%)\end{array}$ & $\begin{array}{r}105 \\
(4.73 \%)\end{array}$ & $\begin{array}{r}2091 \\
(94.19 \%)\end{array}$ & $\begin{array}{r}0 \\
(0.00 \%)\end{array}$ & $\begin{array}{r}24 \\
(1.08 \%)\end{array}$ \\
\hline 12 & 197557 & $\begin{array}{r}1717 \\
(0.87 \%)\end{array}$ & $\begin{array}{r}78553 \\
(39.76 \%)\end{array}$ & 100104 & $12 \rightarrow 13$ & 1149 & $\begin{array}{r}197351 \\
(99.90 \%)\end{array}$ & $\begin{array}{r}167 \\
(0.08 \%)\end{array}$ & 192 & 120 & $\begin{array}{r}205 \\
(0.10 \%)\end{array}$ & $\begin{array}{r}22 \\
(10.73 \%)\end{array}$ & $\begin{array}{r}173 \\
(84.39 \%)\end{array}$ & $\begin{array}{r}0 \\
(0.00 \%)\end{array}$ & $\begin{array}{r}10 \\
(4.88 \%)\end{array}$ \\
\hline 13 & 197574 & $\begin{array}{r}1717 \\
(0.87 \%)\end{array}$ & $\begin{array}{r}78553 \\
(39.76 \%)\end{array}$ & 100115 & $13 \rightarrow 14$ & 2793 & $\begin{array}{r}196875 \\
(99.65 \%)\end{array}$ & $\begin{array}{r}242 \\
(0.12 \%)\end{array}$ & 202 & 394 & $\begin{array}{r}337 \\
(0.17 \%)\end{array}$ & $\begin{array}{r}118 \\
(35.01 \%)\end{array}$ & $\begin{array}{r}200 \\
(59.35 \%)\end{array}$ & $\begin{array}{r}0 \\
(0.00 \%)\end{array}$ & $\begin{array}{r}19 \\
(5.64 \%)\end{array}$ \\
\hline 14 & 197617 & $\begin{array}{r}1717 \\
(0.87 \%)\end{array}$ & $\begin{array}{r}78553 \\
(39.75 \%)\end{array}$ & 100148 & $14 \rightarrow 15$ & 3036 & $\begin{array}{r}196783 \\
(99.58 \%)\end{array}$ & $\begin{array}{r}340 \\
(0.17 \%)\end{array}$ & 207 & 410 & $\begin{array}{r}539 \\
(0.27 \%)\end{array}$ & $\begin{array}{r}216 \\
(40.07 \%)\end{array}$ & $\begin{array}{r}287 \\
(53.25 \%)\end{array}$ & $\begin{array}{r}0 \\
(0.00 \%)\end{array}$ & $\begin{array}{r}36 \\
(6.68 \%)\end{array}$ \\
\hline 15 & 197574 & $\begin{array}{r}1717 \\
(0.87 \%)\end{array}$ & $\begin{array}{r}78553 \\
(39.76 \%)\end{array}$ & 100115 & & & & & & & & & & & \\
\hline
\end{tabular}


Incremental parsing measurements for SDF3 source DynSem.

\begin{tabular}{|c|c|c|c|c|c|c|c|c|c|c|c|c|c|c|c|}
\hline \multirow[b]{2}{*}{ Version } & \multicolumn{3}{|c|}{ Parse Nodes } & \multirow{2}{*}{$\begin{array}{c}\text { Character } \\
\text { Nodes } \\
\text { Count }\end{array}$} & \multirow[b]{2}{*}{ Version } & \multicolumn{3}{|c|}{ Parse Nodes } & \multicolumn{2}{|c|}{ Shift } & \multicolumn{5}{|c|}{ Breakdown } \\
\hline & Count & $\begin{array}{l}\text { Ambi- } \\
\text { guous }\end{array}$ & $\begin{array}{c}\text { Irre- } \\
\text { usable }\end{array}$ & & & Created & Reused & Rebuilt & $\begin{array}{l}\text { Parse } \\
\text { Node }\end{array}$ & $\begin{array}{c}\text { Character } \\
\text { Node }\end{array}$ & Count & $\begin{array}{l}\text { Contains } \\
\text { Change }\end{array}$ & $\begin{array}{c}\text { Irre- } \\
\text { usable }\end{array}$ & $\begin{array}{c}\text { No } \\
\text { Actions }\end{array}$ & $\begin{array}{l}\text { Wrong } \\
\text { State }\end{array}$ \\
\hline $\begin{array}{c}\text { Average } \\
(0 . .14)\end{array}$ & 20549 & $\begin{array}{r}253 \\
(1.23 \%) \\
\end{array}$ & $\begin{array}{r}8237 \\
(40.08 \%) \\
\end{array}$ & 9680 & $\begin{array}{c}\text { Average } \\
(1 . .15)\end{array}$ & 2200 & $\begin{array}{r}20169 \\
(98.15 \%) \\
\end{array}$ & $\begin{array}{r}256 \\
(1.25 \%) \\
\end{array}$ & 132 & 300 & $\begin{array}{r}353 \\
(1.72 \%) \\
\end{array}$ & $\begin{array}{r}75 \\
(20.64 \%) \\
\end{array}$ & $\begin{array}{r}273 \\
(78.00 \%) \\
\end{array}$ & $\begin{array}{r}0 \\
(0.00 \%) \\
\end{array}$ & $\begin{array}{r}5 \\
(1.36 \%) \\
\end{array}$ \\
\hline & & & & & $\rightarrow 0$ & 50935 & & & 0 & 9070 & & & & & \\
\hline 0 & 19331 & $\begin{array}{r}239 \\
(1.24 \%)\end{array}$ & $\begin{array}{r}7763 \\
(40.16 \%)\end{array}$ & 9066 & $0 \rightarrow 1$ & 4425 & $\begin{array}{r}18864 \\
(97.58 \%)\end{array}$ & $\begin{array}{r}349 \\
(1.81 \%)\end{array}$ & 180 & 655 & $\begin{array}{r}466 \\
(2.41 \%)\end{array}$ & $\begin{array}{r}86 \\
(18.45 \%)\end{array}$ & $\begin{array}{r}373 \\
(80.04 \%)\end{array}$ & $\begin{array}{r}0 \\
(0.00 \%)\end{array}$ & $\begin{array}{r}7 \\
(1.50 \%)\end{array}$ \\
\hline 1 & 20227 & $\begin{array}{r}252 \\
(1.25 \%)\end{array}$ & $\begin{array}{r}8091 \\
(40.00 \%)\end{array}$ & 9540 & $1 \rightarrow 2$ & 2218 & $\begin{array}{r}19750 \\
(97.64 \%)\end{array}$ & $\begin{array}{r}340 \\
(1.68 \%)\end{array}$ & 196 & 200 & $\begin{array}{r}476 \\
(2.35 \%)\end{array}$ & $\begin{array}{r}86 \\
(18.07 \%)\end{array}$ & $\begin{array}{r}387 \\
(81.30 \%)\end{array}$ & $\begin{array}{r}0 \\
(0.00 \%)\end{array}$ & $\begin{array}{r}3 \\
(0.63 \%)\end{array}$ \\
\hline 2 & 20241 & $\begin{array}{r}252 \\
(1.24 \%)\end{array}$ & $\begin{array}{r}8088 \\
(39.96 \%)\end{array}$ & 9550 & $2 \rightarrow 3$ & 1061 & $\begin{array}{r}20056 \\
(99.09 \%)\end{array}$ & $\begin{array}{r}155 \\
(0.77 \%)\end{array}$ & 85 & 167 & $\begin{array}{r}186 \\
(0.92 \%)\end{array}$ & $\begin{array}{r}32 \\
(17.20 \%)\end{array}$ & $\begin{array}{r}148 \\
(79.57 \%)\end{array}$ & $\begin{array}{r}0 \\
(0.00 \%)\end{array}$ & $\begin{array}{r}6 \\
(3.23 \%)\end{array}$ \\
\hline 3 & 20231 & $\begin{array}{r}251 \\
(1.24 \%)\end{array}$ & $\begin{array}{r}8083 \\
(39.95 \%)\end{array}$ & 9546 & $3 \rightarrow 4$ & 1875 & $\begin{array}{r}19851 \\
(98.12 \%)\end{array}$ & $\begin{array}{r}288 \\
(1.42 \%)\end{array}$ & 133 & 165 & $\begin{array}{r}379 \\
(1.87 \%)\end{array}$ & $\begin{array}{r}44 \\
(11.61 \%)\end{array}$ & $\begin{array}{r}333 \\
(87.86 \%)\end{array}$ & $\begin{array}{r}0 \\
(0.00 \%)\end{array}$ & $\begin{array}{r}2 \\
(0.53 \%)\end{array}$ \\
\hline 4 & 20233 & $\begin{array}{r}251 \\
(1.24 \%)\end{array}$ & $\begin{array}{r}8083 \\
(39.95 \%)\end{array}$ & 9548 & $4 \rightarrow 5$ & 1857 & $\begin{array}{r}19847 \\
(98.09 \%)\end{array}$ & $\begin{array}{r}326 \\
(1.61 \%)\end{array}$ & 138 & 174 & $\begin{array}{r}387 \\
(1.91 \%)\end{array}$ & $\begin{array}{r}42 \\
(10.85 \%)\end{array}$ & $\begin{array}{r}331 \\
(85.53 \%)\end{array}$ & $\begin{array}{r}0 \\
(0.00 \%)\end{array}$ & $\begin{array}{r}14 \\
(3.62 \%)\end{array}$ \\
\hline 5 & 20222 & $\begin{array}{r}250 \\
(1.24 \%)\end{array}$ & $\begin{array}{r}8077 \\
(39.94 \%)\end{array}$ & 9544 & $5 \rightarrow 6$ & 1493 & $\begin{array}{r}19835 \\
(98.09 \%)\end{array}$ & $\begin{array}{r}217 \\
(1.07 \%)\end{array}$ & 124 & 225 & $\begin{array}{r}384 \\
(1.90 \%)\end{array}$ & $\begin{array}{r}176 \\
(45.83 \%)\end{array}$ & $\begin{array}{r}204 \\
(53.13 \%)\end{array}$ & $\begin{array}{r}0 \\
(0.00 \%)\end{array}$ & $\begin{array}{r}4 \\
(1.04 \%)\end{array}$ \\
\hline 6 & 20119 & $\begin{array}{r}249 \\
(1.24 \%)\end{array}$ & $\begin{array}{r}8008 \\
(39.80 \%)\end{array}$ & 9470 & $6 \rightarrow 7$ & 3458 & $\begin{array}{r}19538 \\
(97.11 \%)\end{array}$ & $\begin{array}{r}334 \\
(1.66 \%)\end{array}$ & 211 & 540 & $\begin{array}{r}478 \\
(2.38 \%)\end{array}$ & $\begin{array}{r}109 \\
(22.80 \%)\end{array}$ & $\begin{array}{r}368 \\
(76.99 \%)\end{array}$ & $\begin{array}{r}0 \\
(0.00 \%)\end{array}$ & $\begin{array}{r}1 \\
(0.21 \%)\end{array}$ \\
\hline 7 & 20438 & $\begin{array}{r}254 \\
(1.24 \%)\end{array}$ & $\begin{array}{r}8160 \\
(39.93 \%)\end{array}$ & 9652 & $7 \rightarrow 8$ & 1718 & $\begin{array}{r}20260 \\
(99.13 \%)\end{array}$ & $\begin{array}{r}135 \\
(0.66 \%)\end{array}$ & 93 & 285 & $\begin{array}{r}177 \\
(0.87 \%)\end{array}$ & $\begin{array}{r}21 \\
(11.86 \%)\end{array}$ & $\begin{array}{r}152 \\
(85.88 \%)\end{array}$ & $\begin{array}{r}0 \\
(0.00 \%)\end{array}$ & $\begin{array}{r}4 \\
(2.26 \%)\end{array}$ \\
\hline 8 & 20683 & $\begin{array}{r}255 \\
(1.23 \%)\end{array}$ & $\begin{array}{r}8285 \\
(40.06 \%)\end{array}$ & 9759 & $8 \rightarrow 9$ & 903 & $\begin{array}{r}20140 \\
(97.37 \%)\end{array}$ & $\begin{array}{r}148 \\
(0.72 \%)\end{array}$ & 78 & 173 & $\begin{array}{r}223 \\
(1.08 \%)\end{array}$ & $\begin{array}{r}104 \\
(46.64 \%)\end{array}$ & $\begin{array}{r}118 \\
(52.91 \%)\end{array}$ & $\begin{array}{r}0 \\
(0.00 \%)\end{array}$ & $\begin{array}{r}1 \\
(0.45 \%)\end{array}$ \\
\hline 9 & 20304 & $\begin{array}{r}249 \\
(1.23 \%)\end{array}$ & $\begin{array}{r}8119 \\
(39.99 \%)\end{array}$ & 9550 & $9 \rightarrow 10$ & 1789 & $\begin{array}{r}20146 \\
(99.22 \%)\end{array}$ & $\begin{array}{r}121 \\
(0.60 \%)\end{array}$ & 82 & 323 & $\begin{array}{r}157 \\
(0.77 \%)\end{array}$ & $\begin{array}{r}20 \\
(12.74 \%)\end{array}$ & $\begin{array}{r}136 \\
(86.62 \%)\end{array}$ & $\begin{array}{r}0 \\
(0.00 \%)\end{array}$ & $\begin{array}{r}1 \\
(0.64 \%)\end{array}$ \\
\hline 10 & 20650 & $\begin{array}{r}251 \\
(1.22 \%)\end{array}$ & $\begin{array}{r}8275 \\
(40.07 \%)\end{array}$ & 9711 & $10 \rightarrow 11$ & 4180 & $\begin{array}{r}20061 \\
(97.15 \%)\end{array}$ & $\begin{array}{r}398 \\
(1.93 \%)\end{array}$ & 159 & 603 & $\begin{array}{r}605 \\
(2.93 \%)\end{array}$ & $\begin{array}{r}180 \\
(29.75 \%)\end{array}$ & $\begin{array}{r}407 \\
(67.27 \%)\end{array}$ & $\begin{array}{r}0 \\
(0.00 \%)\end{array}$ & $\begin{array}{r}18 \\
(2.98 \%)\end{array}$ \\
\hline 11 & 21180 & $\begin{array}{r}256 \\
(1.21 \%)\end{array}$ & $\begin{array}{r}8540 \\
(40.32 \%)\end{array}$ & 9968 & $11 \rightarrow 12$ & 1736 & $\begin{array}{r}20926 \\
(98.80 \%)\end{array}$ & $\begin{array}{r}192 \\
(0.91 \%)\end{array}$ & 101 & 263 & $\begin{array}{r}253 \\
(1.19 \%)\end{array}$ & $\begin{array}{r}36 \\
(14.23 \%)\end{array}$ & $\begin{array}{r}210 \\
(83.00 \%)\end{array}$ & $\begin{array}{r}0 \\
(0.00 \%)\end{array}$ & $\begin{array}{r}7 \\
(2.77 \%)\end{array}$ \\
\hline 12 & 21312 & $\begin{array}{r}259 \\
(1.22 \%)\end{array}$ & $\begin{array}{r}8600 \\
(40.35 \%)\end{array}$ & 10027 & $12 \rightarrow 13$ & 2133 & $\begin{array}{r}20826 \\
(97.72 \%)\end{array}$ & $\begin{array}{r}367 \\
(1.72 \%)\end{array}$ & 166 & 199 & $\begin{array}{r}487 \\
(2.29 \%)\end{array}$ & $\begin{array}{r}80 \\
(16.43 \%)\end{array}$ & $\begin{array}{r}406 \\
(83.37 \%)\end{array}$ & $\begin{array}{r}0 \\
(0.00 \%)\end{array}$ & $\begin{array}{r}1 \\
(0.21 \%)\end{array}$ \\
\hline 13 & 21312 & $\begin{array}{r}259 \\
(1.22 \%)\end{array}$ & $\begin{array}{r}8602 \\
(40.36 \%)\end{array}$ & 10027 & $13 \rightarrow 14$ & 2630 & $\begin{array}{r}20990 \\
(98.49 \%)\end{array}$ & $\begin{array}{r}234 \\
(1.10 \%)\end{array}$ & 148 & 360 & $\begin{array}{r}321 \\
(1.51 \%)\end{array}$ & $\begin{array}{r}66 \\
(20.56 \%)\end{array}$ & $\begin{array}{r}254 \\
(79.13 \%)\end{array}$ & $\begin{array}{r}0 \\
(0.00 \%)\end{array}$ & $\begin{array}{r}1 \\
(0.31 \%)\end{array}$ \\
\hline 14 & 21750 & $\begin{array}{r}267 \\
(1.23 \%)\end{array}$ & $\begin{array}{r}8785 \\
(40.39 \%)\end{array}$ & 10248 & $14 \rightarrow 15$ & 1525 & $\begin{array}{r}21440 \\
(98.57 \%)\end{array}$ & $\begin{array}{r}239 \\
(1.10 \%)\end{array}$ & 93 & 166 & $\begin{array}{r}311 \\
(1.43 \%)\end{array}$ & $\begin{array}{r}39 \\
(12.54 \%)\end{array}$ & $\begin{array}{r}272 \\
(87.46 \%)\end{array}$ & $\begin{array}{r}0 \\
(0.00 \%)\end{array}$ & $\begin{array}{r}0 \\
(0.00 \%)\end{array}$ \\
\hline 15 & 21750 & $\begin{array}{r}267 \\
(1.23 \%)\end{array}$ & $\begin{array}{r}8785 \\
(40.39 \%)\end{array}$ & 10248 & & & & & & & & & & & \\
\hline
\end{tabular}


Incremental parsing measurements for SDF3 source FlowSpec.

\begin{tabular}{|c|c|c|c|c|c|c|c|c|c|c|c|c|c|c|c|}
\hline \multirow[b]{2}{*}{ Version } & \multicolumn{3}{|c|}{ Parse Nodes } & \multirow{2}{*}{$\begin{array}{c}\text { Character } \\
\text { Nodes } \\
\text { Count }\end{array}$} & \multirow[b]{2}{*}{ Version } & \multicolumn{3}{|c|}{ Parse Nodes } & \multicolumn{2}{|c|}{ Shift } & \multicolumn{5}{|c|}{ Breakdown } \\
\hline & Count & $\begin{array}{l}\text { Ambi- } \\
\text { guous }\end{array}$ & $\begin{array}{c}\text { Irre- } \\
\text { usable }\end{array}$ & & & Created & Reused & Rebuilt & $\begin{array}{l}\text { Parse } \\
\text { Node }\end{array}$ & $\begin{array}{c}\text { Character } \\
\text { Node }\end{array}$ & Count & $\begin{array}{l}\text { Contains } \\
\text { Change }\end{array}$ & $\begin{array}{c}\text { Irre- } \\
\text { usable }\end{array}$ & $\begin{array}{c}\text { No } \\
\text { Actions }\end{array}$ & $\begin{array}{l}\text { Wrong } \\
\text { State }\end{array}$ \\
\hline $\begin{array}{c}\text { Average } \\
(0 . .14)\end{array}$ & 24064 & $\begin{array}{r}259 \\
(1.07 \%)\end{array}$ & $\begin{array}{r}11733 \\
(48.77 \%)\end{array}$ & 12079 & $\begin{array}{c}\text { Average } \\
(1 . .15)\end{array}$ & 6187 & $\begin{array}{r}22937 \\
(95.14 \%)\end{array}$ & $\begin{array}{r}695 \\
(3.04 \%)\end{array}$ & 254 & 798 & $\begin{array}{r}925 \\
(4.05 \%)\end{array}$ & $\begin{array}{r}211 \\
(21.18 \%)\end{array}$ & $\begin{array}{r}695 \\
(77.06 \%)\end{array}$ & $\begin{array}{r}0 \\
(0.00 \%)\end{array}$ & $\begin{array}{r}19 \\
(1.77 \%)\end{array}$ \\
\hline & & & & & $\rightarrow 0$ & 59603 & & & 0 & 10180 & & & & & \\
\hline 0 & 19941 & $\begin{array}{r}210 \\
(1.05 \%)\end{array}$ & $\begin{array}{r}9753 \\
(48.91 \%)\end{array}$ & 10166 & $0 \rightarrow 1$ & 10431 & $\begin{array}{r}17770 \\
(89.11 \%)\end{array}$ & $\begin{array}{r}1408 \\
(7.06 \%)\end{array}$ & 474 & 1169 & $\begin{array}{r}2072 \\
(10.39 \%)\end{array}$ & $\begin{array}{r}533 \\
(25.72 \%)\end{array}$ & $\begin{array}{r}1520 \\
(73.36 \%)\end{array}$ & $\begin{array}{r}0 \\
(0.00 \%)\end{array}$ & $\begin{array}{r}19 \\
(0.92 \%)\end{array}$ \\
\hline 1 & 20465 & $\begin{array}{r}215 \\
(1.05 \%)\end{array}$ & $\begin{array}{r}9920 \\
(48.47 \%)\end{array}$ & 10398 & $1 \rightarrow 2$ & 5162 & $\begin{array}{r}19646 \\
(96.00 \%)\end{array}$ & $\begin{array}{r}653 \\
(3.19 \%)\end{array}$ & 271 & 583 & $\begin{array}{r}816 \\
(3.99 \%)\end{array}$ & $\begin{array}{r}103 \\
(12.62 \%)\end{array}$ & $\begin{array}{r}691 \\
(84.68 \%)\end{array}$ & $\begin{array}{r}0 \\
(0.00 \%)\end{array}$ & $\begin{array}{r}22 \\
(2.70 \%)\end{array}$ \\
\hline 2 & 20853 & $\begin{array}{r}219 \\
(1.05 \%)\end{array}$ & $\begin{array}{r}10127 \\
(48.56 \%)\end{array}$ & 10584 & $2 \rightarrow 3$ & 7087 & $\begin{array}{r}19710 \\
(94.52 \%)\end{array}$ & $\begin{array}{r}820 \\
(3.93 \%)\end{array}$ & 254 & 883 & $\begin{array}{r}1150 \\
(5.51 \%)\end{array}$ & $\begin{array}{r}246 \\
(21.39 \%)\end{array}$ & $\begin{array}{r}889 \\
(77.30 \%)\end{array}$ & $\begin{array}{r}0 \\
(0.00 \%)\end{array}$ & $\begin{array}{r}15 \\
(1.30 \%)\end{array}$ \\
\hline 3 & 21591 & $\begin{array}{r}230 \\
(1.07 \%)\end{array}$ & $\begin{array}{r}10563 \\
(48.92 \%)\end{array}$ & 10931 & $3 \rightarrow 4$ & 14153 & $\begin{array}{r}19357 \\
(89.65 \%)\end{array}$ & $\begin{array}{r}1738 \\
(8.05 \%)\end{array}$ & 605 & 1656 & $\begin{array}{r}2227 \\
(10.31 \%)\end{array}$ & $\begin{array}{r}384 \\
(17.24 \%)\end{array}$ & $\begin{array}{r}1787 \\
(80.24 \%)\end{array}$ & $\begin{array}{r}0 \\
(0.00 \%)\end{array}$ & $\begin{array}{r}56 \\
(2.51 \%)\end{array}$ \\
\hline 4 & 23044 & $\begin{array}{r}248 \\
(1.08 \%)\end{array}$ & $\begin{array}{r}11312 \\
(49.09 \%)\end{array}$ & 11530 & $4 \rightarrow 5$ & 7615 & $\begin{array}{r}22006 \\
(95.50 \%)\end{array}$ & $\begin{array}{r}852 \\
(3.70 \%)\end{array}$ & 355 & 845 & $\begin{array}{r}1036 \\
(4.50 \%)\end{array}$ & $\begin{array}{r}111 \\
(10.71 \%)\end{array}$ & $\begin{array}{r}891 \\
(86.00 \%)\end{array}$ & $\begin{array}{r}0 \\
(0.00 \%)\end{array}$ & $\begin{array}{r}34 \\
(3.28 \%)\end{array}$ \\
\hline 5 & 23832 & $\begin{array}{r}263 \\
(1.10 \%)\end{array}$ & $\begin{array}{r}11690 \\
(49.05 \%)\end{array}$ & 11891 & $5 \rightarrow 6$ & 8917 & $\begin{array}{r}22702 \\
(95.26 \%)\end{array}$ & $\begin{array}{r}797 \\
(3.34 \%)\end{array}$ & 408 & 1140 & $\begin{array}{r}1139 \\
(4.78 \%)\end{array}$ & $\begin{array}{r}338 \\
(29.68 \%)\end{array}$ & $\begin{array}{r}764 \\
(67.08 \%)\end{array}$ & $\begin{array}{r}0 \\
(0.00 \%)\end{array}$ & $\begin{array}{r}37 \\
(3.25 \%)\end{array}$ \\
\hline 6 & 25088 & $\begin{array}{r}275 \\
(1.10 \%)\end{array}$ & $\begin{array}{r}12362 \\
(49.27 \%)\end{array}$ & 12522 & $6 \rightarrow 7$ & 6956 & $\begin{array}{r}23570 \\
(93.95 \%)\end{array}$ & $\begin{array}{r}1113 \\
(4.44 \%)\end{array}$ & 426 & 785 & $\begin{array}{r}1439 \\
(5.74 \%)\end{array}$ & $\begin{array}{r}261 \\
(18.14 \%)\end{array}$ & $\begin{array}{r}1120 \\
(77.83 \%)\end{array}$ & $\begin{array}{r}0 \\
(0.00 \%)\end{array}$ & $\begin{array}{r}58 \\
(4.03 \%)\end{array}$ \\
\hline 7 & 25250 & $\begin{array}{r}275 \\
(1.09 \%)\end{array}$ & $\begin{array}{r}12372 \\
(49.00 \%)\end{array}$ & 12580 & $7 \rightarrow 8$ & 920 & $\begin{array}{r}24957 \\
(98.84 \%)\end{array}$ & $\begin{array}{r}218 \\
(0.86 \%)\end{array}$ & 86 & 116 & $\begin{array}{r}268 \\
(1.06 \%)\end{array}$ & $\begin{array}{r}85 \\
(31.72 \%)\end{array}$ & $\begin{array}{r}183 \\
(68.28 \%)\end{array}$ & $\begin{array}{r}0 \\
(0.00 \%)\end{array}$ & $\begin{array}{r}0 \\
(0.00 \%)\end{array}$ \\
\hline 8 & 25181 & $\begin{array}{r}273 \\
(1.08 \%)\end{array}$ & $\begin{array}{r}12345 \\
(49.03 \%)\end{array}$ & 12551 & $8 \rightarrow 9$ & 926 & $\begin{array}{r}24994 \\
(99.26 \%)\end{array}$ & $\begin{array}{r}133 \\
(0.53 \%)\end{array}$ & 62 & 114 & $\begin{array}{r}186 \\
(0.74 \%)\end{array}$ & $\begin{array}{r}30 \\
(16.13 \%)\end{array}$ & $\begin{array}{r}154 \\
(82.80 \%)\end{array}$ & $\begin{array}{r}0 \\
(0.00 \%)\end{array}$ & $\begin{array}{r}2 \\
(1.08 \%)\end{array}$ \\
\hline 9 & 25239 & $\begin{array}{r}273 \\
(1.08 \%)\end{array}$ & $\begin{array}{r}12369 \\
(49.01 \%)\end{array}$ & 12582 & $9 \rightarrow 10$ & 1948 & $\begin{array}{r}24817 \\
(98.33 \%)\end{array}$ & $\begin{array}{r}393 \\
(1.56 \%)\end{array}$ & 122 & 282 & $\begin{array}{r}421 \\
(1.67 \%)\end{array}$ & $\begin{array}{r}11 \\
(2.61 \%)\end{array}$ & $\begin{array}{r}395 \\
(93.82 \%)\end{array}$ & $\begin{array}{r}0 \\
(0.00 \%)\end{array}$ & $\begin{array}{r}15 \\
(3.56 \%)\end{array}$ \\
\hline 10 & 25304 & $\begin{array}{r}274 \\
(1.08 \%)\end{array}$ & $\begin{array}{r}12402 \\
(49.01 \%)\end{array}$ & 12620 & $10 \rightarrow 11$ & 670 & $\begin{array}{r}25216 \\
(99.65 \%)\end{array}$ & $\begin{array}{r}56 \\
(0.22 \%)\end{array}$ & 63 & 86 & $\begin{array}{r}87 \\
(0.34 \%)\end{array}$ & $\begin{array}{r}21 \\
(24.14 \%)\end{array}$ & $\begin{array}{r}66 \\
(75.86 \%)\end{array}$ & $\begin{array}{r}0 \\
(0.00 \%)\end{array}$ & $\begin{array}{r}0 \\
(0.00 \%)\end{array}$ \\
\hline 11 & 25373 & $\begin{array}{r}275 \\
(1.08 \%)\end{array}$ & $\begin{array}{r}12435 \\
(49.01 \%)\end{array}$ & 12659 & $11 \rightarrow 12$ & 857 & $\begin{array}{r}25179 \\
(99.24 \%)\end{array}$ & $\begin{array}{r}141 \\
(0.56 \%)\end{array}$ & 69 & 94 & $\begin{array}{r}201 \\
(0.79 \%)\end{array}$ & $\begin{array}{r}52 \\
(25.87 \%)\end{array}$ & $\begin{array}{r}147 \\
(73.13 \%)\end{array}$ & $\begin{array}{r}0 \\
(0.00 \%)\end{array}$ & $\begin{array}{r}2 \\
(1.00 \%)\end{array}$ \\
\hline 12 & 25368 & $\begin{array}{r}275 \\
(1.08 \%)\end{array}$ & $\begin{array}{r}12430 \\
(49.00 \%)\end{array}$ & 12654 & $12 \rightarrow 13$ & 20204 & $\begin{array}{r}20515 \\
(80.87 \%)\end{array}$ & $\begin{array}{r}1437 \\
(5.66 \%)\end{array}$ & 427 & 3248 & $\begin{array}{r}2037 \\
(8.03 \%)\end{array}$ & $\begin{array}{r}863 \\
(42.37 \%)\end{array}$ & $\begin{array}{r}1156 \\
(56.75 \%)\end{array}$ & $\begin{array}{r}0 \\
(0.00 \%)\end{array}$ & $\begin{array}{r}18 \\
(0.88 \%)\end{array}$ \\
\hline 13 & 26820 & $\begin{array}{r}283 \\
(1.06 \%)\end{array}$ & $\begin{array}{r}12771 \\
(47.62 \%)\end{array}$ & 13540 & $13 \rightarrow 14$ & 5218 & $\begin{array}{r}26220 \\
(97.76 \%)\end{array}$ & $\begin{array}{r}530 \\
(1.98 \%)\end{array}$ & 98 & 693 & $\begin{array}{r}599 \\
(2.23 \%)\end{array}$ & $\begin{array}{r}63 \\
(10.52 \%)\end{array}$ & $\begin{array}{r}530 \\
(88.48 \%)\end{array}$ & $\begin{array}{r}0 \\
(0.00 \%)\end{array}$ & $\begin{array}{r}6 \\
(1.00 \%)\end{array}$ \\
\hline 14 & 27616 & $\begin{array}{r}295 \\
(1.07 \%)\end{array}$ & $\begin{array}{r}13137 \\
(47.57 \%)\end{array}$ & 13980 & $14 \rightarrow 15$ & 1741 & $\begin{array}{r}27397 \\
(99.21 \%)\end{array}$ & $\begin{array}{r}143 \\
(0.52 \%)\end{array}$ & 92 & 280 & $\begin{array}{r}198 \\
(0.72 \%)\end{array}$ & $\begin{array}{r}57 \\
(28.79 \%)\end{array}$ & $\begin{array}{r}139 \\
(70.20 \%)\end{array}$ & $(0.00 \%)$ & $\begin{array}{r}2 \\
(1.01 \%)\end{array}$ \\
\hline 15 & 28023 & $\begin{array}{r}295 \\
(1.05 \%)\end{array}$ & $\begin{array}{r}13248 \\
(47.28 \%)\end{array}$ & 14154 & & & & & & & & & & & \\
\hline
\end{tabular}


Incremental parsing measurements for SDF3 source Stratego.

\begin{tabular}{|c|c|c|c|c|c|c|c|c|c|c|c|c|c|c|c|}
\hline \multirow[b]{2}{*}{ Version } & \multicolumn{3}{|c|}{ Parse Nodes } & \multirow{2}{*}{$\begin{array}{c}\text { Character } \\
\text { Nodes } \\
\text { Count }\end{array}$} & \multirow[b]{2}{*}{ Version } & \multicolumn{3}{|c|}{ Parse Nodes } & \multicolumn{2}{|c|}{ Shift } & \multicolumn{5}{|c|}{ Breakdown } \\
\hline & Count & $\begin{array}{l}\text { Ambi- } \\
\text { guous }\end{array}$ & $\begin{array}{c}\text { Irre- } \\
\text { usable }\end{array}$ & & & Created & Reused & Rebuilt & $\begin{array}{l}\text { Parse } \\
\text { Node }\end{array}$ & $\begin{array}{c}\text { Character } \\
\text { Node }\end{array}$ & Count & $\begin{array}{l}\text { Contains } \\
\text { Change }\end{array}$ & $\begin{array}{c}\text { Irre- } \\
\text { usable }\end{array}$ & $\begin{array}{c}\text { No } \\
\text { Actions }\end{array}$ & $\begin{array}{l}\text { Wrong } \\
\text { State }\end{array}$ \\
\hline $\begin{array}{c}\text { Average } \\
(9 . .14)\end{array}$ & 151528 & $\begin{array}{r}1094 \\
(0.72 \%) \\
\end{array}$ & $\begin{array}{r}55800 \\
(36.82 \%) \\
\end{array}$ & 79123 & $\begin{array}{c}\text { Average } \\
(10 . .15) \\
\end{array}$ & 6874 & $\begin{array}{r}150400 \\
(99.25 \%) \\
\end{array}$ & $\begin{array}{r}933 \\
(0.62 \%) \\
\end{array}$ & 303 & 864 & $\begin{array}{r}1108 \\
(0.73 \%) \\
\end{array}$ & $\begin{array}{r}171 \\
(24.81 \%) \\
\end{array}$ & $\begin{array}{r}879 \\
(69.59 \%) \\
\end{array}$ & $\begin{array}{r}0 \\
(0.00 \%) \\
\end{array}$ & $\begin{array}{r}58 \\
(5.60 \%) \\
\end{array}$ \\
\hline & & & & & $\rightarrow 9$ & 401368 & & & 0 & 78354 & & & & & \\
\hline 9 & 150091 & $\begin{array}{r}1080 \\
(0.72 \%)\end{array}$ & $\begin{array}{r}55045 \\
(36.67 \%)\end{array}$ & 78291 & $9 \rightarrow 10$ & 402 & $\begin{array}{r}149947 \\
(99.90 \%)\end{array}$ & $\begin{array}{r}31 \\
(0.02 \%)\end{array}$ & 74 & 122 & $\begin{array}{r}132 \\
(0.09 \%)\end{array}$ & $\begin{array}{r}110 \\
(83.33 \%)\end{array}$ & $\begin{array}{r}22 \\
(16.67 \%)\end{array}$ & $\begin{array}{r}0 \\
(0.00 \%)\end{array}$ & $\begin{array}{r}0 \\
(0.00 \%)\end{array}$ \\
\hline 10 & 150138 & $\begin{array}{r}1080 \\
(0.72 \%)\end{array}$ & $\begin{array}{r}55087 \\
(36.69 \%)\end{array}$ & 78333 & $10 \rightarrow 11$ & 19726 & $\begin{array}{r}147456 \\
(98.21 \%)\end{array}$ & $\begin{array}{r}2250 \\
(1.50 \%)\end{array}$ & 476 & 2558 & $\begin{array}{r}2696 \\
(1.80 \%)\end{array}$ & $\begin{array}{r}323 \\
(11.98 \%)\end{array}$ & $\begin{array}{r}2330 \\
(86.42 \%)\end{array}$ & $\begin{array}{r}0 \\
(0.00 \%)\end{array}$ & $\begin{array}{r}43 \\
(1.59 \%)\end{array}$ \\
\hline 11 & 151550 & $\begin{array}{r}1096 \\
(0.72 \%)\end{array}$ & $\begin{array}{r}55773 \\
(36.80 \%)\end{array}$ & 79231 & $11 \rightarrow 12$ & 2406 & $\begin{array}{r}151382 \\
(99.89 \%)\end{array}$ & $\begin{array}{r}153 \\
(0.10 \%)\end{array}$ & 109 & 209 & $\begin{array}{r}167 \\
(0.11 \%)\end{array}$ & $\begin{array}{r}15 \\
(8.98 \%)\end{array}$ & $\begin{array}{r}127 \\
(76.05 \%)\end{array}$ & $\begin{array}{r}0 \\
(0.00 \%)\end{array}$ & $\begin{array}{r}25 \\
(14.97 \%)\end{array}$ \\
\hline 12 & 151908 & $\begin{array}{r}1097 \\
(0.72 \%)\end{array}$ & $\begin{array}{r}55978 \\
(36.85 \%)\end{array}$ & 79378 & $12 \rightarrow 13$ & 4650 & $\begin{array}{r}151137 \\
(99.49 \%)\end{array}$ & $\begin{array}{r}572 \\
(0.38 \%)\end{array}$ & 231 & 634 & $\begin{array}{r}750 \\
(0.49 \%)\end{array}$ & $\begin{array}{r}109 \\
(14.53 \%)\end{array}$ & $\begin{array}{r}590 \\
(78.67 \%)\end{array}$ & $\begin{array}{r}0 \\
(0.00 \%)\end{array}$ & $\begin{array}{r}51 \\
(6.80 \%)\end{array}$ \\
\hline 13 & 152430 & $\begin{array}{r}1100 \\
(0.72 \%)\end{array}$ & $\begin{array}{r}56287 \\
(36.93 \%)\end{array}$ & 79672 & $13 \rightarrow 14$ & 13306 & $\begin{array}{r}149645 \\
(98.17 \%)\end{array}$ & $\begin{array}{r}2431 \\
(1.59 \%)\end{array}$ & 804 & 1562 & $\begin{array}{r}2681 \\
(1.76 \%)\end{array}$ & $\begin{array}{r}438 \\
(16.34 \%)\end{array}$ & $\begin{array}{r}2018 \\
(75.27 \%)\end{array}$ & $\begin{array}{r}0 \\
(0.00 \%)\end{array}$ & $\begin{array}{r}225 \\
(8.39 \%)\end{array}$ \\
\hline 14 & 153053 & $\begin{array}{r}1108 \\
(0.72 \%)\end{array}$ & $\begin{array}{r}56632 \\
(37.00 \%)\end{array}$ & 79832 & $14 \rightarrow 15$ & 756 & $\begin{array}{r}152835 \\
(99.86 \%)\end{array}$ & $\begin{array}{r}163 \\
(0.11 \%)\end{array}$ & 125 & 96 & $\begin{array}{r}219 \\
(0.14 \%)\end{array}$ & $\begin{array}{r}30 \\
(13.70 \%)\end{array}$ & $\begin{array}{r}185 \\
(84.47 \%)\end{array}$ & $\begin{array}{r}0 \\
(0.00 \%)\end{array}$ & $\begin{array}{r}4 \\
(1.83 \%)\end{array}$ \\
\hline 15 & 153054 & $\begin{array}{r}1108 \\
(0.72 \%)\end{array}$ & $\begin{array}{r}56633 \\
(37.00 \%)\end{array}$ & 79833 & & & & & & & & & & & \\
\hline
\end{tabular}


Incremental parsing measurements for SDF3 source WebDSL.

\begin{tabular}{|c|c|c|c|c|c|c|c|c|c|c|c|c|c|c|c|}
\hline \multirow[b]{2}{*}{ Version } & \multicolumn{3}{|c|}{ Parse Nodes } & \multirow{2}{*}{$\begin{array}{c}\text { Character } \\
\text { Nodes } \\
\text { Count }\end{array}$} & \multirow[b]{2}{*}{ Version } & \multicolumn{3}{|c|}{ Parse Nodes } & \multicolumn{2}{|c|}{ Shift } & \multicolumn{5}{|c|}{ Breakdown } \\
\hline & Count & $\begin{array}{l}\text { Ambi- } \\
\text { guous }\end{array}$ & $\begin{array}{c}\text { Irre- } \\
\text { usable }\end{array}$ & & & Created & Reused & Rebuilt & $\begin{array}{l}\text { Parse } \\
\text { Node }\end{array}$ & $\begin{array}{c}\text { Character } \\
\text { Node }\end{array}$ & Count & $\begin{array}{l}\text { Contains } \\
\text { Change }\end{array}$ & $\begin{array}{c}\text { Irre- } \\
\text { usable }\end{array}$ & $\begin{array}{c}\text { No } \\
\text { Actions }\end{array}$ & $\begin{array}{c}\text { Wrong } \\
\text { State }\end{array}$ \\
\hline $\begin{array}{c}\text { Average } \\
(0 . .14)\end{array}$ & 162581 & $\begin{array}{r}1853 \\
(1.14 \%)\end{array}$ & $\begin{array}{r}67285 \\
(41.39 \%) \\
\end{array}$ & 85998 & $\begin{array}{c}\text { Average } \\
(1 . .15)\end{array}$ & 6191 & $\begin{array}{r}161117 \\
(99.10 \%)\end{array}$ & $\begin{array}{r}1287 \\
(0.79 \%)\end{array}$ & 334 & 1017 & $\begin{array}{r}1444 \\
(0.89 \%)\end{array}$ & $\begin{array}{r}99 \\
(11.37 \%)\end{array}$ & $\begin{array}{r}1331 \\
(87.58 \%)\end{array}$ & $\begin{array}{r}0 \\
(0.00 \%)\end{array}$ & $\begin{array}{r}14 \\
(1.05 \%)\end{array}$ \\
\hline & & & & & $\rightarrow 0$ & 422079 & & & 0 & 85526 & & & & & \\
\hline 0 & 161682 & $\begin{array}{r}1829 \\
(1.13 \%)\end{array}$ & $\begin{array}{r}67013 \\
(41.45 \%)\end{array}$ & 85500 & $0 \rightarrow 1$ & 1074 & $\begin{array}{r}161420 \\
(99.84 \%)\end{array}$ & $\begin{array}{r}87 \\
(0.05 \%)\end{array}$ & 82 & 169 & $\begin{array}{r}263 \\
(0.16 \%)\end{array}$ & $\begin{array}{r}175 \\
(66.54 \%)\end{array}$ & $\begin{array}{r}88 \\
(33.46 \%)\end{array}$ & $\begin{array}{r}0 \\
(0.00 \%)\end{array}$ & $\begin{array}{r}0 \\
(0.00 \%)\end{array}$ \\
\hline 1 & 161735 & $\begin{array}{r}1829 \\
(1.13 \%)\end{array}$ & $\begin{array}{r}67020 \\
(41.44 \%)\end{array}$ & 85515 & $1 \rightarrow 2$ & 2491 & $\begin{array}{r}161488 \\
(99.85 \%)\end{array}$ & $\begin{array}{r}190 \\
(0.12 \%)\end{array}$ & 115 & 426 & $\begin{array}{r}246 \\
(0.15 \%)\end{array}$ & $\begin{array}{r}34 \\
(13.82 \%)\end{array}$ & $\begin{array}{r}211 \\
(85.77 \%)\end{array}$ & $\begin{array}{r}0 \\
(0.00 \%)\end{array}$ & $\begin{array}{r}1 \\
(0.41 \%)\end{array}$ \\
\hline 2 & 162255 & $\begin{array}{r}1837 \\
(1.13 \%)\end{array}$ & $\begin{array}{r}67200 \\
(41.42 \%)\end{array}$ & 85783 & $2 \rightarrow 3$ & 16085 & $\begin{array}{r}158693 \\
(97.80 \%)\end{array}$ & $\begin{array}{r}3212 \\
(1.98 \%)\end{array}$ & 967 & 2217 & $\begin{array}{r}3558 \\
(2.19 \%)\end{array}$ & $\begin{array}{r}131 \\
(3.68 \%)\end{array}$ & $\begin{array}{r}3382 \\
(95.05 \%)\end{array}$ & $\begin{array}{r}0 \\
(0.00 \%)\end{array}$ & $\begin{array}{r}45 \\
(1.26 \%)\end{array}$ \\
\hline 3 & 162345 & $\begin{array}{r}1838 \\
(1.13 \%)\end{array}$ & $\begin{array}{r}67260 \\
(41.43 \%)\end{array}$ & 85846 & $3 \rightarrow 4$ & 7615 & $\begin{array}{r}160514 \\
(98.87 \%)\end{array}$ & $\begin{array}{r}1704 \\
(1.05 \%)\end{array}$ & 434 & 1254 & $\begin{array}{r}1830 \\
(1.13 \%)\end{array}$ & $\begin{array}{r}53 \\
(2.90 \%)\end{array}$ & $\begin{array}{r}1771 \\
(96.78 \%)\end{array}$ & $\begin{array}{r}0 \\
(0.00 \%)\end{array}$ & $\begin{array}{r}6 \\
(0.33 \%)\end{array}$ \\
\hline 4 & 162361 & $\begin{array}{r}1838 \\
(1.13 \%)\end{array}$ & $\begin{array}{r}67260 \\
(41.43 \%)\end{array}$ & 85856 & $4 \rightarrow 5$ & 8149 & $\begin{array}{r}160343 \\
(98.76 \%)\end{array}$ & $\begin{array}{r}1771 \\
(1.09 \%)\end{array}$ & 446 & 1323 & $\begin{array}{r}1982 \\
(1.22 \%)\end{array}$ & $\begin{array}{r}153 \\
(7.72 \%)\end{array}$ & $\begin{array}{r}1821 \\
(91.88 \%)\end{array}$ & $\begin{array}{r}0 \\
(0.00 \%)\end{array}$ & $\begin{array}{r}8 \\
(0.40 \%)\end{array}$ \\
\hline 5 & 162369 & $\begin{array}{r}1841 \\
(1.13 \%)\end{array}$ & $\begin{array}{r}67220 \\
(41.40 \%)\end{array}$ & 85844 & $5 \rightarrow 6$ & 4912 & $\begin{array}{r}161016 \\
(99.17 \%)\end{array}$ & $\begin{array}{r}1196 \\
(0.74 \%)\end{array}$ & 277 & 861 & $\begin{array}{r}1277 \\
(0.79 \%)\end{array}$ & $\begin{array}{r}73 \\
(5.72 \%)\end{array}$ & $\begin{array}{r}1196 \\
(93.66 \%)\end{array}$ & $\begin{array}{r}0 \\
(0.00 \%)\end{array}$ & $\begin{array}{r}8 \\
(0.63 \%)\end{array}$ \\
\hline 6 & 162273 & $\begin{array}{r}1840 \\
(1.13 \%)\end{array}$ & $\begin{array}{r}67184 \\
(41.40 \%)\end{array}$ & 85799 & $6 \rightarrow 7$ & 8917 & $\begin{array}{r}159740 \\
(98.44 \%)\end{array}$ & $\begin{array}{r}1845 \\
(1.14 \%)\end{array}$ & 479 & 1655 & $\begin{array}{r}2399 \\
(1.48 \%)\end{array}$ & $\begin{array}{r}457 \\
(19.05 \%)\end{array}$ & $\begin{array}{r}1874 \\
(78.12 \%)\end{array}$ & $\begin{array}{r}0 \\
(0.00 \%)\end{array}$ & $\begin{array}{r}68 \\
(2.83 \%)\end{array}$ \\
\hline 7 & 162457 & $\begin{array}{r}1856 \\
(1.14 \%)\end{array}$ & $\begin{array}{r}67210 \\
(41.37 \%)\end{array}$ & 85931 & $7 \rightarrow 8$ & 16962 & $\begin{array}{r}158781 \\
(97.74 \%)\end{array}$ & $\begin{array}{r}3490 \\
(2.15 \%)\end{array}$ & 528 & 2532 & $\begin{array}{r}3675 \\
(2.26 \%)\end{array}$ & $\begin{array}{r}29 \\
(0.79 \%)\end{array}$ & $\begin{array}{r}3642 \\
(99.10 \%)\end{array}$ & $\begin{array}{r}0 \\
(0.00 \%)\end{array}$ & $\begin{array}{r}4 \\
(0.11 \%)\end{array}$ \\
\hline 8 & 162615 & $\begin{array}{r}1857 \\
(1.14 \%)\end{array}$ & $\begin{array}{r}67304 \\
(41.39 \%)\end{array}$ & 86050 & $8 \rightarrow 9$ & 2066 & $\begin{array}{r}162256 \\
(99.78 \%)\end{array}$ & $\begin{array}{r}326 \\
(0.20 \%)\end{array}$ & 110 & 375 & $\begin{array}{r}358 \\
(0.22 \%)\end{array}$ & $\begin{array}{r}23 \\
(6.42 \%)\end{array}$ & $\begin{array}{r}334 \\
(93.30 \%)\end{array}$ & $\begin{array}{r}0 \\
(0.00 \%)\end{array}$ & $\begin{array}{r}1 \\
(0.28 \%)\end{array}$ \\
\hline 9 & 162878 & $\begin{array}{r}1864 \\
(1.14 \%)\end{array}$ & $\begin{array}{r}67403 \\
(41.38 \%)\end{array}$ & 86167 & $9 \rightarrow 10$ & 7783 & $\begin{array}{r}160967 \\
(98.83 \%)\end{array}$ & $\begin{array}{r}1751 \\
(1.08 \%)\end{array}$ & 445 & 1263 & $\begin{array}{r}1897 \\
(1.16 \%)\end{array}$ & $\begin{array}{r}67 \\
(3.53 \%)\end{array}$ & $\begin{array}{r}1826 \\
(96.26 \%)\end{array}$ & $\begin{array}{r}0 \\
(0.00 \%)\end{array}$ & $\begin{array}{r}4 \\
(0.21 \%)\end{array}$ \\
\hline 10 & 162841 & $\begin{array}{r}1864 \\
(1.14 \%)\end{array}$ & $\begin{array}{r}67376 \\
(41.38 \%)\end{array}$ & 86154 & $10 \rightarrow 11$ & 2079 & $\begin{array}{r}162477 \\
(99.78 \%)\end{array}$ & $\begin{array}{r}332 \\
(0.20 \%)\end{array}$ & 108 & 398 & $\begin{array}{r}363 \\
(0.22 \%)\end{array}$ & $\begin{array}{r}18 \\
(4.96 \%)\end{array}$ & $\begin{array}{r}345 \\
(95.04 \%)\end{array}$ & $\begin{array}{r}0 \\
(0.00 \%)\end{array}$ & $\begin{array}{r}0 \\
(0.00 \%)\end{array}$ \\
\hline 11 & 163063 & $\begin{array}{r}1868 \\
(1.15 \%)\end{array}$ & $\begin{array}{r}67436 \\
(41.36 \%)\end{array}$ & 86285 & $11 \rightarrow 12$ & 2814 & $\begin{array}{r}162257 \\
(99.51 \%)\end{array}$ & $\begin{array}{r}595 \\
(0.36 \%)\end{array}$ & 189 & 500 & $\begin{array}{r}797 \\
(0.49 \%)\end{array}$ & $\begin{array}{r}149 \\
(18.70 \%)\end{array}$ & $\begin{array}{r}607 \\
(76.16 \%)\end{array}$ & $\begin{array}{r}0 \\
(0.00 \%)\end{array}$ & $\begin{array}{r}41 \\
(5.14 \%)\end{array}$ \\
\hline 12 & 163158 & $\begin{array}{r}1876 \\
(1.15 \%)\end{array}$ & $\begin{array}{r}67445 \\
(41.34 \%)\end{array}$ & 86330 & $12 \rightarrow 13$ & 1632 & $\begin{array}{r}162778 \\
(99.77 \%)\end{array}$ & $\begin{array}{r}324 \\
(0.20 \%)\end{array}$ & 132 & 281 & $\begin{array}{r}379 \\
(0.23 \%)\end{array}$ & $\begin{array}{r}34 \\
(8.97 \%)\end{array}$ & $\begin{array}{r}331 \\
(87.34 \%)\end{array}$ & $\begin{array}{r}0 \\
(0.00 \%)\end{array}$ & $\begin{array}{r}14 \\
(3.69 \%)\end{array}$ \\
\hline 13 & 163210 & $\begin{array}{r}1876 \\
(1.15 \%)\end{array}$ & $\begin{array}{r}67445 \\
(41.32 \%)\end{array}$ & 86370 & $13 \rightarrow 14$ & 1606 & $\begin{array}{r}162913 \\
(99.82 \%)\end{array}$ & $\begin{array}{r}279 \\
(0.17 \%)\end{array}$ & 99 & 412 & $\begin{array}{r}296 \\
(0.18 \%)\end{array}$ & $\begin{array}{r}13 \\
(4.39 \%)\end{array}$ & $\begin{array}{r}283 \\
(95.61 \%)\end{array}$ & $\begin{array}{r}0 \\
(0.00 \%)\end{array}$ & $\begin{array}{r}0 \\
(0.00 \%)\end{array}$ \\
\hline 14 & 163466 & $\begin{array}{r}1876 \\
(1.15 \%)\end{array}$ & $\begin{array}{r}67495 \\
(41.29 \%)\end{array}$ & 86543 & $14 \rightarrow 15$ & 8683 & $\begin{array}{r}161111 \\
(98.56 \%)\end{array}$ & $\begin{array}{r}2208 \\
(1.35 \%)\end{array}$ & 605 & 1591 & $\begin{array}{r}2337 \\
(1.43 \%)\end{array}$ & $\begin{array}{r}78 \\
(3.34 \%)\end{array}$ & $\begin{array}{r}2248 \\
(96.19 \%)\end{array}$ & $\begin{array}{r}0 \\
(0.00 \%)\end{array}$ & $\begin{array}{r}11 \\
(0.47 \%)\end{array}$ \\
\hline 15 & 163466 & $\begin{array}{r}1876 \\
(1.15 \%)\end{array}$ & $\begin{array}{r}67495 \\
(41.29 \%)\end{array}$ & 86538 & & & & & & & & & & & \\
\hline
\end{tabular}




\section{는 $\quad$ A.2 Time Benchmarks}

The tables on the left show parse times for only the parsing phase, while the tables on the right show parse times for the full JSGLR2 parsing pipeline, which includes imploding and tokenization, as shown in the bottom row of Figures 2.10 and 3.12. All times are measured in milliseconds. The tables in the middle show the size of the input and the number of added/removed characters for each version.

Average parse time for all languages, excluding version 0.

\begin{tabular}{|c|c|c|c|c|c|c|c|c|c|c|c|c|c|c|}
\hline Language & Standard & Elkhound & $\begin{array}{r}\text { Incremental } \\
\text { (no cache) }\end{array}$ & Incremental & $\begin{array}{r}\text { Size } \\
\text { (B) }\end{array}$ & $\begin{array}{r}\text { Removed } \\
\text { (B) }\end{array}$ & $\begin{array}{r}\text { Added } \\
\text { (B) }\end{array}$ & Language & Standard & Elkhound & $\begin{array}{r}\text { Incremental } \\
\text { (no cache) }\end{array}$ & Incremental & $\begin{array}{l}\text { Tree-sitter } \\
\text { (no cache) }\end{array}$ & Tree-sitter \\
\hline Average & .510 & 235 & 839.299 & 82.50 & 6635 & 682 & 816 & Average & 810.266 & 630. & 1007.738 & 90.302 & - & - \\
\hline Java & 1974.375 & 1497.341 & 2112.104 & 203.043 & 851080 & 1757 & 1725 & Java & 2033.161 & 1579.048 & 2518.010 & 217.591 & 233.929 & 19.715 \\
\hline WebDSL & 233.029 & 175.540 & 244.903 & 23.681 & 101548 & 205 & 458 & WebDSL & 241.125 & 185.905 & 303.368 & 27.597 & - & - \\
\hline SDF3 & 149.127 & 118.824 & 160.888 & 20.784 & 57277 & 83 & 265 & SDF3 & 156.514 & 127.480 & 201.835 & 25.719 & - & - \\
\hline
\end{tabular}

\section{A.2.1 Java}

Average parse times for the Java language, excluding version 0 .

\begin{tabular}{|c|c|c|c|c|c|c|c|c|c|c|c|c|c|c|}
\hline Source & Standard & Elkhound & $\begin{array}{r}\text { Incremental } \\
\text { (no cache) }\end{array}$ & Incremental & $\begin{array}{r}\text { Size } \\
\text { (B) }\end{array}$ & $\begin{array}{r}\text { Removed } \\
\text { (B) }\end{array}$ & $\begin{array}{r}\text { Added } \\
\text { (B) }\end{array}$ & Source & Standard & Elkhound & $\begin{array}{r}\text { Incremental } \\
\text { (no cache) }\end{array}$ & Incremental & $\begin{array}{l}\text { Tree-sitter } \\
\text { (no cache) }\end{array}$ & Tree-sitter \\
\hline Average & \begin{tabular}{|l|}
1974.375 \\
\end{tabular} & 1497.341 & 2112.104 & 203.043 & 851080 & 1757 & 1725 & Average & 2033.161 & 1579.048 & 2518.010 & 217.591 & 233.929 & 19.715 \\
\hline StringUtils & 548.470 & 406.090 & 576.152 & 163.664 & 396376 & 369 & 481 & StringUtils & 580.587 & 436.086 & 692.993 & 170.913 & 54.080 & 21.040 \\
\hline gson & 3530.745 & 2748.975 & 3775.883 & 289.502 & 1265099 & 344 & 644 & gson & 3626.562 & 2866.369 & 4530.654 & 301.643 & 434.953 & 20.072 \\
\hline slf4j & 1843.908 & 1336.959 & 1984.278 & 155.963 & 891764 & 4557 & 4049 & slf4j & 1892.333 & 1434.687 & 2330.383 & 180.218 & 212.755 & 18.034 \\
\hline
\end{tabular}




\section{Parse times for Java source StringUtils.}

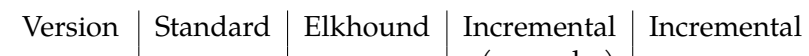

Size $\mid$ Removed $\mid$ Added

\begin{tabular}{r|r|r}
$(\mathrm{B})$ & $(\mathrm{B})$ & $(\mathrm{B})$ \\
\hline 396376 & 369 & 481 \\
\hline 395110 & - & - \\
395172 & 0 & 62 \\
395216 & 0 & 44 \\
394856 & 515 & 155 \\
397653 & 0 & 2797 \\
395931 & 3940 & 2218 \\
395896 & 35 & 0 \\
395842 & 64 & 10 \\
395842 & 7 & 7 \\
397158 & 0 & 1316 \\
397176 & 0 & 18 \\
397184 & 0 & 8 \\
397048 & 136 & 0 \\
397077 & 0 & 29 \\
396792 & 754 & 469 \\
396796 & 80 & 84
\end{tabular}

\begin{tabular}{l|l|l|l|l|l|l} 
Version & Standard & Elkhound & Incremental & Incremental & Tree-sitter & Tree-sitter
\end{tabular}

\begin{tabular}{c|r|r|r|r|r|r}
\hline Average & 580.587 & 436.086 & (no cache) & & (no cache) & \\
\hline 0 & 613.234 & 447.702 & 685.152 & 670.913 & 54.080 & 21.040 \\
1 & 589.096 & 430.918 & 665.578 & 204.115 & 53.953 & 55.989 \\
2 & 525.333 & 442.995 & 670.220 & 133.418 & 54.245 & 5.190 \\
3 & 596.581 & 444.350 & 702.911 & 253.706 & 55.092 & 54.311 \\
4 & 598.532 & 442.535 & 673.724 & 175.444 & 54.990 & 5.662 \\
5 & 589.638 & 431.712 & 749.065 & 296.903 & 53.480 & 77.045 \\
6 & 553.568 & 435.171 & 710.847 & 44.800 & 53.708 & 2.056 \\
7 & 580.305 & 429.797 & 683.152 & 109.176 & 53.764 & 5.572 \\
8 & 557.878 & 431.062 & 684.287 & 110.730 & 54.037 & 5.479 \\
9 & 554.663 & 426.705 & 678.664 & 67.274 & 53.735 & 5.411 \\
10 & 625.021 & 437.813 & 685.739 & 151.585 & 54.321 & 6.441 \\
11 & 587.075 & 443.293 & 678.918 & 220.436 & 53.922 & 11.363 \\
12 & 577.435 & 442.426 & 733.281 & 198.861 & 53.780 & 23.409 \\
13 & 598.358 & 440.583 & 670.686 & 96.101 & 53.761 & 16.372 \\
14 & 620.832 & 429.453 & 690.750 & 309.318 & 54.291 & 71.858 \\
15 & 554.494 & 432.485 & 717.076 & 191.827 & 53.704 & 10.500
\end{tabular}


Parse times for Java source gson.

Version $\mid$ Standard $\mid$ Elkhound $\mid$ Incremental $\mid$ Incremental

\begin{tabular}{r|r|r}
$\begin{array}{r}\text { Size } \\
(\mathrm{B})\end{array}$ & $\begin{array}{r}\text { Removed } \\
(\mathrm{B})\end{array}$ & $\begin{array}{r}\text { Added } \\
(\mathrm{B})\end{array}$ \\
\hline 1265099 & 344 & 644 \\
\hline 1263538 & 0 & 1263518 \\
1263386 & 573 & 421 \\
1263310 & 98 & 22 \\
1263306 & 24 & 20 \\
1262894 & 1083 & 671 \\
1262894 & 0 & 0 \\
1263022 & 0 & 126 \\
1262846 & 302 & 126 \\
1263220 & 0 & 374 \\
1263298 & 25 & 103 \\
1267896 & 0 & 4598 \\
1268060 & 0 & 164 \\
1268144 & 0 & 84 \\
1267762 & 1135 & 753 \\
1268412 & 788 & 1438 \\
1268030 & 1135 & 753
\end{tabular}

\begin{tabular}{c|r|r|r|r|r|r} 
Version & Standard & Elkhound & $\begin{array}{r}\text { Incremental } \\
\text { (no cache) }\end{array}$ & Incremental & $\begin{array}{r}\text { Tree-sitter } \\
\text { (no cache) }\end{array}$ & Tree-sitter \\
\hline Average & 3626.562 & 2866.369 & 4530.654 & 301.643 & 434.953 & 20.072 \\
\hline 0 & 3533.697 & 2877.887 & 4453.189 & 4786.196 & 432.136 & 443.473 \\
1 & 3278.285 & 2699.250 & 4267.664 & 303.627 & 447.886 & 19.127 \\
2 & 3822.314 & 2858.514 & 4484.484 & 289.680 & 438.720 & 16.571 \\
3 & 3386.830 & 2883.233 & 4454.842 & 293.020 & 431.946 & 19.386 \\
4 & 3821.525 & 2875.712 & 4656.754 & 353.911 & 433.808 & 34.124 \\
5 & 3816.020 & 2878.528 & 4804.442 & 294.436 & 434.877 & 15.768 \\
6 & 3900.653 & 2859.448 & 4307.176 & 288.156 & 431.076 & 20.862 \\
7 & 3797.019 & 2912.520 & 4339.391 & 307.115 & 439.001 & 21.316 \\
8 & 3459.621 & 3009.576 & 4827.296 & 285.400 & 431.967 & 18.220 \\
9 & 3361.746 & 2857.024 & 4297.740 & 299.716 & 429.407 & 20.830 \\
10 & 3464.597 & 2823.515 & 4449.837 & 309.913 & 433.013 & 21.895 \\
11 & 3370.940 & 2872.003 & 4797.996 & 289.960 & 436.452 & 18.685 \\
12 & 3820.651 & 2819.557 & 4560.324 & 290.045 & 434.129 & 18.649 \\
13 & 3864.571 & 2873.621 & 4452.984 & 293.021 & 436.982 & 18.472 \\
14 & 3822.406 & 2944.329 & 4553.043 & 312.105 & 433.934 & 19.199 \\
15 & 3411.260 & 2828.712 & 4705.833 & 314.536 & 431.090 & 17.972
\end{tabular}


Parse times for Java source slf4j.

\begin{tabular}{|c|c|c|c|c|c|c|c|c|c|c|c|c|c|c|}
\hline Version & Standard & Elkhound & $\begin{array}{r}\text { Incremental } \\
\text { (no cache) }\end{array}$ & Incremental & $\begin{array}{r}\text { Size } \\
\text { (B) }\end{array}$ & $\begin{array}{r}\text { Removed } \\
\text { (B) }\end{array}$ & $\begin{array}{r}\text { Added } \\
\text { (B) }\end{array}$ & Version & Standard & Elkhound & $\begin{array}{r}\text { Incremental } \\
\text { (no cache) }\end{array}$ & Incremental & $\begin{array}{l}\text { Tree-sitter } \\
\text { (no cache) }\end{array}$ & Tree-sitter \\
\hline Average & 1843.908 & 1336.959 & 1984.278 & 155.963 & 891764 & 4557 & 4049 & Average & 1892.333 & 1434.687 & 2330.383 & 180.218 & 212.755 & 18.034 \\
\hline 0 & 1950.869 & 1396.467 & 1924.703 & 1895.252 & 912784 & - & - & 0 & 1962.037 & 1461.973 & 2301.925 & 2429.781 & 217.761 & 219.257 \\
\hline 1 & 1914.720 & 1345.672 & 2191.663 & 160.343 & 912785 & 0 & 1 & 1 & 1949.528 & 1453.325 & 2256.715 & 172.429 & 217.246 & 8.911 \\
\hline 2 & 1910.553 & 1395.761 & 1905.263 & 135.988 & 912813 & 8 & 36 & 2 & 1780.389 & 1411.431 & 2238.574 & 143.523 & 216.544 & 10.035 \\
\hline 3 & 1688.898 & 1418.170 & 1927.477 & 140.715 & 912814 & 0 & 1 & 3 & 2086.934 & 1446.944 & 2467.858 & 151.674 & 219.741 & 10.524 \\
\hline 4 & 1952.318 & 1273.339 & 2138.546 & 178.288 & 913852 & 8814 & 9852 & 4 & 1937.893 & 1484.835 & 2524.441 & 268.604 & 218.903 & 25.982 \\
\hline 5 & 1913.849 & 1302.889 & 1963.789 & 225.093 & 902914 & 20902 & 9964 & 5 & 2009.672 & 1558.417 & 2273.049 & 326.079 & 215.636 & 67.605 \\
\hline 6 & 1887.770 & 1391.150 & 1883.856 & 135.516 & 902912 & 6 & 4 & 6 & 1942.201 & 1467.338 & 2490.050 & 142.852 & 214.497 & 10.747 \\
\hline 7 & 1977.712 & 1361.097 & 1914.908 & 226.041 & 863562 & 37270 & 19602 & 7 & 1741.697 & 1503.599 & 2298.376 & 290.557 & 212.140 & 41.282 \\
\hline 8 & 1625.192 & 1370.718 & 2067.581 & 180.761 & 881191 & 671 & 18300 & 8 & 1853.447 & 1406.083 & 2182.388 & 201.929 & 208.579 & 20.808 \\
\hline 9 & 1879.117 & 1250.072 & 1861.661 & 132.147 & 881184 & 7 & 0 & 9 & 1728.795 & 1394.955 & 2292.012 & 137.419 & 209.333 & 10.251 \\
\hline 10 & 1851.934 & 1343.630 & 2096.098 & 135.887 & 881132 & 52 & 0 & 10 & 1897.723 & 1409.310 & 2211.121 & 140.060 & 207.715 & 10.656 \\
\hline 11 & 1853.402 & 1242.876 & 1855.218 & 145.600 & 881134 & 5 & 7 & 11 & 1993.814 & 1397.318 & 2475.115 & 153.019 & 210.503 & 8.233 \\
\hline 12 & 1878.930 & 1329.713 & 2131.885 & 134.209 & 881518 & 16 & 400 & 12 & 1911.829 & 1446.683 & 2280.729 & 143.075 & 208.636 & 11.411 \\
\hline 13 & 1883.879 & 1331.258 & 1904.392 & 135.011 & 881618 & 2 & 102 & 13 & 1833.444 & 1434.646 & 2300.400 & 136.817 & 209.983 & 7.986 \\
\hline 14 & 1856.527 & 1327.615 & 1775.771 & 138.090 & 883547 & 202 & 2131 & 14 & 1701.047 & 1295.931 & 2489.464 & 151.905 & 210.005 & 13.943 \\
\hline 15 & 1583.828 & 1370.429 & 2146.060 & 135.751 & 883490 & 399 & 342 & 15 & 2016.578 & 1409.487 & 2175.459 & 143.323 & 211.867 & 12.134 \\
\hline
\end{tabular}

\section{A.2.2 WebDSL}

Average parse times for the WebDSL language, excluding version 0.

\begin{tabular}{|c|c|c|c|c|c|c|c|c|c|c|c|c|}
\hline Source & Standard & Elkhound & $\begin{array}{r}\text { Incremental } \\
\text { (no cache) }\end{array}$ & Incremental & $\begin{array}{r}\text { Size } \\
\text { (B) }\end{array}$ & $\begin{array}{r}\text { Removed } \\
\text { (B) }\end{array}$ & $\begin{array}{r}\text { Added } \\
\text { (B) }\end{array}$ & Source & Standard & Elkhound & $\begin{array}{r}\text { Incremental } \\
\text { (no cache) }\end{array}$ & Incremental \\
\hline Average & 233.029 & 175.540 & 244.903 & 23.681 & 101548 & 205 & 458 & Average & 241.125 & 185.905 & 303.368 & 27.597 \\
\hline builtin.app & 249.465 & 185.023 & 263.670 & 27.441 & 97843 & 475 & 174 & builtin.app & 267.130 & 196.868 & 334.089 & 31.030 \\
\hline YellowGrass & 360.934 & 270.288 & 375.227 & 34.731 & 167287 & 107 & 1060 & YellowGrass & 364.214 & 285.688 & 458.316 & 40.419 \\
\hline elib-utils & 88.690 & 71.310 & 95.813 & 8.871 & 39515 & 32 & 141 & elib-utils & 92.029 & 75.159 & 117.699 & 11.342 \\
\hline
\end{tabular}


Parse times for WebDSL source builtin.app.

\begin{tabular}{|c|c|c|c|c|c|c|c|c|c|c|c|c|}
\hline Version & Standard & Elkhound & $\begin{array}{r}\text { Incremental } \\
\text { (no cache) }\end{array}$ & Incremental & $\begin{array}{r}\text { Size } \\
(\mathrm{B})\end{array}$ & $\begin{array}{r}\text { Removed } \\
\text { (B) }\end{array}$ & $\begin{array}{r}\text { Added } \\
\text { (B) }\end{array}$ & Version & Standard & Elkhound & $\begin{array}{r}\text { Incremental } \\
\text { (no cache) }\end{array}$ & Incremental \\
\hline Average & 249.465 & 185.023 & 263.670 & 27.441 & 97843 & 475 & 174 & Average & 267.130 & 196.868 & 334.089 & 31.030 \\
\hline 1 & 266.187 & 191.839 & 278.880 & 270.626 & 100882 & - & - & 1 & 279.817 & 196.008 & 340.347 & 342.084 \\
\hline 2 & 262.139 & 185.533 & 277.219 & 25.195 & 97219 & 3663 & 0 & 2 & 268.534 & 202.189 & 324.073 & 26.479 \\
\hline 3 & 223.751 & 179.368 & 262.086 & 27.702 & 98047 & 0 & 828 & 3 & 279.992 & 202.742 & 344.977 & 38.476 \\
\hline 4 & 248.952 & 180.288 & 259.710 & 26.949 & 98231 & 10 & 194 & 4 & 263.362 & 187.933 & 352.829 & 28.644 \\
\hline 5 & 250.799 & 192.804 & 254.144 & 36.115 & 97627 & 604 & 0 & 5 & 261.398 & 191.781 & 332.648 & 45.938 \\
\hline 6 & 256.824 & 182.942 & 259.105 & 24.456 & 97683 & 0 & 56 & 6 & 265.544 & 191.989 & 317.260 & 25.824 \\
\hline 7 & 244.860 & 184.720 & 279.631 & 26.242 & 97721 & 0 & 38 & 7 & 271.876 & 201.561 & 351.438 & 28.053 \\
\hline 8 & 246.218 & 187.573 & 268.891 & 27.521 & 97935 & 0 & 214 & 8 & 262.419 & 196.931 & 326.250 & 29.291 \\
\hline 9 & 260.944 & 184.491 & 252.059 & 25.631 & 97956 & 0 & 21 & 9 & 264.730 & 202.284 & 333.181 & 27.165 \\
\hline 10 & 250.694 & 187.492 & 260.181 & 27.155 & 98172 & 1 & 217 & 10 & 266.317 & 194.397 & 324.146 & 29.401 \\
\hline
\end{tabular}


Parse times for WebDSL source YellowGrass.

\begin{tabular}{|c|c|c|c|c|c|c|c|c|c|c|c|c|}
\hline Version & Standard & Elkhound & $\begin{array}{r}\text { Incremental } \\
\text { (no cache) }\end{array}$ & Incremental & $\begin{array}{r}\text { Size } \\
\text { (B) }\end{array}$ & $\begin{array}{r}\text { Removed } \\
\text { (B) }\end{array}$ & $\begin{array}{r}\text { Added } \\
\text { (B) }\end{array}$ & Version & Standard & Elkhound & $\begin{array}{r}\text { Incremental } \\
\text { (no cache) }\end{array}$ & Incremental \\
\hline Average & 360.934 & 270.288 & 375.227 & 34.731 & 167287 & 107 & 1060 & Average & 364.214 & 285.688 & 458.316 & 40.419 \\
\hline 0 & 326.021 & 258.895 & 382.747 & 375.826 & 158975 & - & - & 0 & 322.583 & 270.795 & 429.477 & 431.878 \\
\hline 1 & 350.203 & 269.790 & 381.338 & 37.656 & 160568 & 121 & 1714 & 1 & 360.298 & 290.884 & 427.663 & 50.274 \\
\hline 2 & 349.087 & 268.371 & 374.891 & 43.228 & 159970 & 954 & 356 & 2 & 318.511 & 269.640 & 434.078 & 61.583 \\
\hline 3 & 350.056 & 256.040 & 348.459 & 32.232 & 160445 & 67 & 542 & 3 & 362.199 & 275.319 & 433.132 & 44.342 \\
\hline 4 & 349.585 & 268.767 & 361.169 & 40.465 & 161720 & 38 & 1313 & 4 & 359.778 & 277.303 & 421.927 & 55.434 \\
\hline 5 & 340.806 & 261.018 & 383.822 & 30.672 & 161716 & 4 & 0 & 5 & 368.950 & 278.087 & 466.042 & 31.967 \\
\hline 6 & 358.853 & 258.436 & 351.155 & 32.425 & 163359 & 0 & 1643 & 6 & 354.989 & 280.019 & 437.152 & 34.685 \\
\hline 7 & 371.666 & 264.268 & 347.987 & 28.763 & 163370 & 12 & 23 & 7 & 372.142 & 274.603 & 437.677 & 30.182 \\
\hline 8 & 371.762 & 274.609 & 393.565 & 40.464 & 168635 & 0 & 5265 & 8 & 335.676 & 286.318 & 469.427 & 44.186 \\
\hline 9 & 368.866 & 277.560 & 352.047 & 39.598 & 172696 & 0 & 4061 & 9 & 358.421 & 297.573 & 446.813 & 43.347 \\
\hline 10 & 369.962 & 280.473 & 399.384 & 29.453 & 172793 & 32 & 129 & 10 & 374.248 & 299.657 & 494.391 & 32.145 \\
\hline 11 & 373.611 & 280.305 & 376.083 & 31.326 & 172833 & 32 & 72 & 11 & 390.910 & 295.862 & 508.930 & 31.577 \\
\hline 12 & 373.763 & 262.900 & 370.201 & 31.007 & 172834 & 0 & 1 & 12 & 389.511 & 289.188 & 452.468 & 31.168 \\
\hline 13 & 331.360 & 275.490 & 408.462 & 31.922 & 172557 & 281 & 4 & 13 & 391.335 & 297.690 & 494.219 & 33.675 \\
\hline 14 & 382.947 & 270.806 & 409.803 & 30.669 & 172529 & 67 & 39 & 14 & 340.434 & 290.551 & 465.037 & 32.109 \\
\hline 15 & 371.483 & 285.486 & 370.034 & 41.082 & 173274 & 0 & 745 & 15 & 385.813 & 282.629 & 485.790 & 49.609 \\
\hline
\end{tabular}


Parse times for WebDSL source elib-utils.

\begin{tabular}{|c|c|c|c|c|c|c|c|c|c|c|c|c|}
\hline Version & Standard & Elkhound & $\begin{array}{r}\text { Incremental } \\
\text { (no cache) }\end{array}$ & Incremental & $\begin{array}{r}\text { Size } \\
(\mathrm{B})\end{array}$ & $\begin{array}{r}\text { Removed } \\
\text { (B) }\end{array}$ & $\begin{array}{r}\text { Added } \\
\text { (B) }\end{array}$ & Version & Standard & Elkhound & $\begin{array}{c}\text { Incremental } \\
\text { (no cache) }\end{array}$ & Incremental \\
\hline Average & 88.690 & 71.310 & 95.813 & 8.871 & 39515 & 32 & 141 & Average & 92.029 & 75.159 & 117.699 & 11.342 \\
\hline 0 & 82.423 & 70.155 & 89.681 & 95.225 & 38955 & - & - & 0 & 96.551 & 71.086 & 110.582 & 114.429 \\
\hline 1 & 87.218 & 68.698 & 98.440 & 7.603 & 38953 & 2 & 0 & 1 & 92.814 & 75.213 & 113.256 & 8.016 \\
\hline 2 & 82.132 & 69.742 & 91.284 & 9.371 & 39118 & 0 & 165 & 2 & 86.217 & 72.883 & 112.333 & 12.733 \\
\hline 3 & 89.226 & 69.606 & 98.881 & 10.049 & 39211 & 66 & 159 & 3 & 91.201 & 73.849 & 122.497 & 13.913 \\
\hline 4 & 89.641 & 71.085 & 87.965 & 8.119 & 39378 & 0 & 167 & 4 & 93.397 & 75.025 & 124.901 & 10.583 \\
\hline 5 & 90.753 & 71.517 & 93.305 & 8.514 & 39317 & 94 & 33 & 5 & 95.497 & 73.691 & 114.172 & 9.534 \\
\hline 6 & 90.943 & 72.859 & 98.665 & 8.662 & 39283 & 48 & 14 & 6 & 93.696 & 77.906 & 119.379 & 11.018 \\
\hline 7 & 92.183 & 70.864 & 95.146 & 8.058 & 39320 & 1 & 38 & 7 & 94.806 & 74.913 & 110.154 & 8.675 \\
\hline 8 & 82.246 & 71.391 & 94.134 & 8.571 & 39302 & 21 & 3 & 8 & 92.301 & 73.878 & 120.606 & 9.304 \\
\hline 9 & 91.406 & 70.044 & 93.005 & 8.001 & 39430 & 0 & 128 & 9 & 93.427 & 74.362 & 117.196 & 8.808 \\
\hline 10 & 90.550 & 72.145 & 94.159 & 8.697 & 39496 & 29 & 95 & 10 & 86.160 & 74.077 & 114.960 & 13.685 \\
\hline 11 & 86.594 & 72.144 & 94.190 & 9.121 & 39480 & 22 & 6 & 11 & 94.842 & 77.152 & 117.428 & 11.032 \\
\hline 12 & 89.672 & 71.642 & 100.388 & 8.367 & 39547 & 2 & 69 & 12 & 93.339 & 74.075 & 114.295 & 9.201 \\
\hline 13 & 83.455 & 71.824 & 93.306 & 8.454 & 39821 & 0 & 274 & 13 & 95.111 & 78.341 & 116.424 & 10.879 \\
\hline 14 & 91.709 & 72.245 & 102.869 & 12.852 & 40480 & 169 & 828 & 14 & 87.856 & 75.412 & 127.476 & 21.259 \\
\hline 15 & 92.622 & 73.845 & 101.460 & 8.625 & 40589 & 22 & 131 & 15 & 89.771 & 76.603 & 120.402 & 11.486 \\
\hline
\end{tabular}

\section{A.2.3 SDF3}

Average parse times for the SDF3 language, excluding version 0.

\begin{tabular}{|c|c|c|c|c|c|c|c|c|c|c|c|c|}
\hline Source & Standard & Elkhound & $\begin{array}{r}\text { Incremental } \\
\text { (no cache) }\end{array}$ & Incremental & $\begin{array}{r}\text { Size } \\
\text { (B) }\end{array}$ & $\begin{array}{r}\text { Removed } \\
\text { (B) }\end{array}$ & $\begin{array}{r}\text { Added } \\
\text { (B) }\end{array}$ & Source & Standard & Elkhound & $\begin{array}{r}\text { Incremental } \\
\text { (no cache) }\end{array}$ & Incremental \\
\hline Average & 149.127 & 118.824 & 160.888 & 20.784 & 57277 & 83 & 265 & Average & 156.514 & 127.480 & 201.835 & 25.719 \\
\hline NaBL & 248.609 & 194.725 & 268.438 & 33.179 & 98834 & 184 & 422 & $\mathrm{NaBL}$ & 254.829 & 209.901 & 330.948 & 38.750 \\
\hline DynSem & 26.006 & 21.133 & 28.662 & 5.145 & 9759 & 25 & 104 & DynSem & 27.797 & 22.963 & 37.408 & 7.857 \\
\hline FlowSpec & 35.278 & 29.035 & 39.252 & 8.577 & 12345 & 125 & 391 & FlowSpec & 37.772 & 31.233 & 50.636 & 13.299 \\
\hline Stratego & 231.338 & 183.947 & 251.471 & 26.424 & 79380 & 61 & 318 & Stratego & 246.166 & 195.066 & 308.393 & 31.794 \\
\hline WebDSL & 204.402 & 165.280 & 216.618 & 30.596 & 86067 & 22 & 92 & WebDSL & 216.004 & 178.237 & 281.788 & 36.896 \\
\hline
\end{tabular}


Parse times for SDF3 source NaBL.

\begin{tabular}{|c|c|c|c|c|c|c|c|c|c|c|c|c|}
\hline Version & Standard & Elkhound & $\begin{array}{r}\text { Incremental } \\
\text { (no cache) }\end{array}$ & Incremental & $\begin{array}{r}\text { Size } \\
\text { (B) }\end{array}$ & $\begin{array}{r}\text { Removed } \\
\text { (B) }\end{array}$ & $\begin{array}{r}\text { Added } \\
\text { (B) }\end{array}$ & Version & Standard & Elkhound & $\begin{array}{r}\text { Incremental } \\
\text { (no cache) }\end{array}$ & Incremental \\
\hline Average & 248.609 & 194.725 & 268.438 & 33.179 & 98834 & 184 & 422 & Average & 254.829 & 209.901 & 330.948 & 38.750 \\
\hline 0 & 250.544 & 192.285 & 271.866 & 249.973 & 96540 & - & - & 0 & 260.355 & 209.091 & 319.534 & 341.853 \\
\hline 1 & 251.955 & 194.495 & 251.967 & 30.628 & 96879 & 0 & 339 & 1 & 265.994 & 214.581 & 317.569 & 33.395 \\
\hline 2 & 240.936 & 193.368 & 255.114 & 28.513 & 96705 & 174 & 0 & 2 & 257.144 & 207.690 & 337.709 & 30.296 \\
\hline 3 & 244.266 & 191.811 & 253.297 & 27.862 & 96706 & 3 & 4 & 3 & 229.444 & 202.478 & 307.133 & 29.336 \\
\hline 4 & 244.303 & 198.509 & 259.455 & 33.187 & 98475 & 341 & 2110 & 4 & 269.891 & 211.170 & 341.943 & 36.723 \\
\hline 5 & 253.614 & 199.143 & 280.289 & 27.593 & 98471 & 4 & 0 & 5 & 263.225 & 203.466 & 342.024 & 28.480 \\
\hline 6 & 254.523 & 187.106 & 287.502 & 28.362 & 98540 & 0 & 69 & 6 & 236.581 & 212.924 & 344.119 & 29.309 \\
\hline 7 & 248.624 & 193.144 & 274.419 & 40.047 & 98556 & 0 & 16 & 7 & 232.504 & 220.040 & 351.583 & 54.574 \\
\hline 8 & 255.298 & 193.190 & 247.621 & 27.635 & 98731 & 0 & 175 & 8 & 237.903 & 211.939 & 303.800 & 29.732 \\
\hline 9 & 242.818 & 200.243 & 260.494 & 28.209 & 98742 & 0 & 11 & 9 & 232.127 & 209.990 & 321.503 & 29.496 \\
\hline 10 & 306.991 & 195.278 & 286.163 & 55.790 & 100108 & 1465 & 2831 & 10 & 270.070 & 209.106 & 352.497 & 73.302 \\
\hline 11 & 256.088 & 190.641 & 284.046 & 42.526 & 100110 & 104 & 106 & 11 & 260.686 & 212.197 & 339.839 & 56.342 \\
\hline 12 & 245.111 & 196.228 & 283.476 & 41.294 & 100104 & 248 & 242 & 12 & 270.553 & 207.202 & 313.593 & 57.086 \\
\hline 13 & 230.777 & 200.295 & 277.397 & 27.543 & 100115 & 0 & 11 & 13 & 273.302 & 205.698 & 349.267 & 28.707 \\
\hline 14 & 232.957 & 192.485 & 250.312 & 29.751 & 100148 & 191 & 224 & 14 & 272.789 & 209.727 & 325.196 & 31.764 \\
\hline 15 & 220.869 & 194.939 & 275.021 & 28.744 & 100115 & 224 & 191 & 15 & 250.227 & 210.304 & 316.438 & 32.708 \\
\hline
\end{tabular}


Parse times for SDF3 source DynSem.

\begin{tabular}{c|r|r|r|r} 
Version & Standard & Elkhound & $\begin{array}{r}\text { Incremental } \\
\text { (no cache) }\end{array}$ & Incremental \\
\hline Average & 26.006 & 21.133 & 28.662 & 5.145 \\
\hline 0 & 23.964 & 19.627 & 26.813 & 26.882 \\
1 & 25.837 & 20.681 & 28.228 & 6.442 \\
2 & 26.099 & 20.172 & 28.725 & 5.290 \\
3 & 25.055 & 20.832 & 27.904 & 4.302 \\
4 & 25.606 & 21.268 & 27.607 & 4.886 \\
5 & 25.833 & 21.062 & 27.942 & 4.962 \\
6 & 25.701 & 20.908 & 28.053 & 4.555 \\
7 & 24.829 & 20.894 & 28.100 & 6.013 \\
8 & 25.709 & 20.468 & 28.012 & 4.584 \\
9 & 24.915 & 21.236 & 27.862 & 4.118 \\
10 & 25.617 & 20.891 & 28.331 & 4.752 \\
11 & 26.959 & 21.202 & 28.452 & 6.502 \\
12 & 26.000 & 21.504 & 29.461 & 4.745 \\
13 & 27.729 & 21.020 & 30.324 & 5.536 \\
14 & 26.929 & 22.194 & 30.200 & 5.621 \\
15 & 27.276 & 22.670 & 30.726 & 4.874
\end{tabular}

\begin{tabular}{r|r|r}
$\begin{array}{r}\text { Size } \\
(\mathrm{B})\end{array}$ & $\begin{array}{r}\text { Removed } \\
(\mathrm{B})\end{array}$ & $\begin{array}{r}\text { Added } \\
(\mathrm{B})\end{array}$ \\
\hline 9759 & 25 & 104 \\
\hline 9066 & - & - \\
9540 & 0 & 474 \\
9550 & 0 & 10 \\
9546 & 4 & 0 \\
9548 & 0 & 2 \\
9544 & 4 & 0 \\
9470 & 78 & 4 \\
9652 & 56 & 238 \\
9759 & 0 & 107 \\
9550 & 209 & 0 \\
9711 & 0 & 161 \\
9968 & 27 & 284 \\
10027 & 0 & 59 \\
10027 & 2 & 2 \\
10248 & 0 & 221 \\
10248 & 2 & 2
\end{tabular}

\begin{tabular}{c|r|r|r|r} 
Version & Standard & Elkhound & $\begin{array}{r}\text { Incremental } \\
\text { (no cache) }\end{array}$ & Incremental \\
\hline Average & 27.797 & 22.963 & 37.408 & 7.857 \\
\hline 0 & 26.048 & 20.996 & 35.466 & 34.575 \\
1 & 26.480 & 22.778 & 34.985 & 11.515 \\
2 & 27.801 & 22.160 & 38.180 & 7.767 \\
3 & 26.280 & 21.637 & 35.854 & 5.443 \\
4 & 26.967 & 22.217 & 36.576 & 6.528 \\
5 & 27.043 & 22.704 & 36.470 & 7.190 \\
6 & 26.993 & 22.661 & 37.548 & 6.234 \\
7 & 27.228 & 22.747 & 35.500 & 9.673 \\
8 & 27.195 & 23.737 & 37.294 & 7.186 \\
9 & 27.946 & 21.747 & 37.103 & 5.161 \\
10 & 27.948 & 22.732 & 37.217 & 7.150 \\
11 & 28.708 & 23.794 & 37.248 & 11.343 \\
12 & 28.030 & 23.470 & 38.551 & 7.021 \\
13 & 28.903 & 23.261 & 38.458 & 8.613 \\
14 & 29.427 & 24.079 & 40.860 & 9.644 \\
15 & 30.001 & 24.714 & 39.279 & 7.395
\end{tabular}


Parse times for SDF3 source FlowSpec.

\begin{tabular}{|c|c|c|c|c|c|c|c|c|c|c|c|c|}
\hline Version & Standard & Elkhound & $\begin{array}{r}\text { Incremental } \\
\text { (no cache) }\end{array}$ & Incremental & $\begin{array}{r}\text { Size } \\
\text { (B) }\end{array}$ & $\begin{array}{r}\text { Removed } \\
\text { (B) }\end{array}$ & $\begin{array}{r}\text { Added } \\
\text { (B) }\end{array}$ & Version & Standard & Elkhound & $\begin{array}{r}\text { Incremental } \\
\text { (no cache) }\end{array}$ & Incremental \\
\hline Average & 35.278 & 29.035 & 39.252 & 8.577 & 12345 & 125 & 391 & Average & 37.772 & 31.233 & 50.636 & 13.299 \\
\hline 0 & 29.827 & 24.026 & 33.622 & 33.251 & 10166 & - & - & 0 & 31.252 & 25.072 & 41.035 & 41.284 \\
\hline 1 & 29.923 & 24.145 & 32.734 & 10.640 & 10398 & 76 & 308 & 1 & 31.045 & 25.964 & 40.726 & 16.745 \\
\hline 2 & 30.332 & 24.278 & 33.491 & 7.185 & 10584 & 0 & 186 & 2 & 31.887 & 26.411 & 40.593 & 10.672 \\
\hline 3 & 31.568 & 26.343 & 33.947 & 8.389 & 10931 & 31 & 378 & 3 & 33.944 & 28.244 & 43.793 & 14.631 \\
\hline 4 & 33.100 & 26.475 & 36.851 & 13.340 & 11530 & 46 & 645 & 4 & 35.116 & 30.201 & 49.271 & 27.023 \\
\hline 5 & 34.484 & 28.446 & 38.581 & 9.606 & 11891 & 0 & 361 & 5 & 36.672 & 30.289 & 49.327 & 15.180 \\
\hline 6 & 35.335 & 29.165 & 40.731 & 10.228 & 12522 & 17 & 648 & 6 & 38.860 & 31.028 & 53.332 & 16.547 \\
\hline 7 & 35.644 & 30.645 & 41.263 & 9.998 & 12580 & 72 & 130 & 7 & 38.130 & 31.224 & 51.229 & 14.669 \\
\hline 8 & 35.244 & 30.601 & 40.648 & 5.494 & 12551 & 29 & 0 & 8 & 38.145 & 31.944 & 54.883 & 6.419 \\
\hline 9 & 37.907 & 29.822 & 40.323 & 5.311 & 12582 & 0 & 31 & 9 & 39.160 & 32.925 & 49.874 & 7.507 \\
\hline 10 & 35.821 & 29.734 & 40.161 & 6.497 & 12620 & 0 & 38 & 10 & 39.768 & 32.681 & 54.161 & 9.018 \\
\hline 11 & 36.921 & 30.450 & 40.689 & 5.377 & 12659 & 0 & 39 & 11 & 38.310 & 31.982 & 51.647 & 6.029 \\
\hline 12 & 36.599 & 29.350 & 40.453 & 5.273 & 12654 & 8 & 3 & 12 & 39.467 & 33.015 & 51.115 & 7.454 \\
\hline 13 & 37.272 & 31.347 & 41.948 & 16.568 & 13540 & 1581 & 2467 & 13 & 41.886 & 33.651 & 53.122 & 23.345 \\
\hline 14 & 37.864 & 31.579 & 42.971 & 8.415 & 13980 & 0 & 440 & 14 & 41.807 & 34.052 & 57.361 & 15.078 \\
\hline 15 & 41.159 & 33.146 & 43.984 & 6.336 & 14154 & 14 & 188 & 15 & 42.385 & 34.883 & 59.102 & 9.165 \\
\hline
\end{tabular}

Parse times for SDF3 source Stratego.

\begin{tabular}{|c|c|c|c|c|c|c|c|c|c|c|c|c|}
\hline Version & Standard & Elkhound & $\begin{array}{r}\text { Incremental } \\
\text { (no cache) }\end{array}$ & Incremental & $\begin{array}{r}\text { Size } \\
(\mathrm{B}) \\
\end{array}$ & $\begin{array}{r}\text { Removed } \\
(\mathrm{B})\end{array}$ & $\begin{array}{r}\text { Added } \\
(\mathrm{B})\end{array}$ & Version & Standard & Elkhound & $\begin{array}{r}\text { Incremental } \\
\text { (no cache) }\end{array}$ & Incremental \\
\hline Average & 231.338 & 183.947 & 251.471 & 26.424 & 79380 & 61 & 318 & Average & 246.166 & 195.066 & 308.393 & 31.794 \\
\hline 9 & 226.619 & 180.479 & 248.538 & 248.358 & 78291 & - & - & 9 & 232.024 & 186.610 & 294.465 & 292.806 \\
\hline 10 & 235.082 & 179.032 & 246.124 & 20.951 & 78333 & 54 & 96 & 10 & 254.045 & 190.244 & 312.593 & 21.307 \\
\hline 11 & 228.249 & 180.503 & 242.531 & 35.282 & 79231 & 162 & 1060 & 11 & 242.430 & 201.798 & 307.034 & 47.601 \\
\hline 12 & 233.428 & 190.932 & 267.120 & 23.903 & 79378 & 0 & 147 & 12 & 245.755 & 191.530 & 300.525 & 25.607 \\
\hline 13 & 213.648 & 183.172 & 251.578 & 23.754 & 79672 & 18 & 312 & 13 & 245.527 & 196.365 & 297.620 & 31.827 \\
\hline 14 & 234.556 & 186.140 & 252.648 & 32.954 & 79832 & 129 & 289 & 14 & 242.960 & 194.322 & 311.118 & 42.320 \\
\hline 15 & 243.065 & 183.902 & 248.824 & 21.699 & 79833 & 2 & 3 & 15 & 246.280 & 196.138 & 321.467 & 22.102 \\
\hline
\end{tabular}


Parse times for SDF3 source WebDSL.

\begin{tabular}{|c|c|c|c|c|c|c|c|}
\hline Version & Standard & Elkhound & $\begin{array}{r}\text { Incremental } \\
\text { (no cache) }\end{array}$ & Incremental & $\begin{array}{r}\text { Size } \\
(\mathrm{B})\end{array}$ & $\begin{array}{r}\text { Removed } \\
\text { (B) }\end{array}$ & $\begin{array}{r}\text { Added } \\
(\mathrm{B})\end{array}$ \\
\hline Average & 204.402 & 165.280 & 216.618 & 30.596 & 86067 & 22 & 92 \\
\hline 0 & 200.194 & 165.581 & 215.281 & 227.837 & 85500 & - & - \\
\hline 1 & 204.197 & 166.431 & 207.700 & 26.615 & 85515 & 52 & 67 \\
\hline 2 & 189.313 & 164.352 & 220.025 & 27.370 & 85783 & 0 & 268 \\
\hline 3 & 197.230 & 164.831 & 207.987 & 39.591 & 85846 & 0 & 63 \\
\hline 4 & 200.683 & 163.307 & 215.856 & 31.261 & 85856 & 0 & 10 \\
\hline 5 & 199.334 & 163.215 & 230.702 & 31.604 & 85844 & 50 & 38 \\
\hline 6 & 207.198 & 166.165 & 218.716 & 29.377 & 85799 & 45 & 0 \\
\hline 7 & 208.579 & 166.837 & 211.050 & 33.062 & 85931 & 114 & 246 \\
\hline 8 & 208.245 & 164.092 & 207.910 & 36.819 & 86050 & 0 & 119 \\
\hline 9 & 206.942 & 164.201 & 209.997 & 26.767 & 86167 & 0 & 117 \\
\hline 10 & 206.028 & 166.243 & 213.583 & 31.967 & 86154 & 17 & 4 \\
\hline 11 & 206.340 & 167.478 & 234.758 & 30.051 & 86285 & 0 & 131 \\
\hline 12 & 215.316 & 163.458 & 209.008 & 27.314 & 86330 & 24 & 69 \\
\hline 13 & 206.024 & 165.222 & 234.109 & 26.943 & 86370 & 0 & 40 \\
\hline 14 & 199.102 & 169.170 & 213.681 & 26.868 & 86543 & 0 & 173 \\
\hline 15 & 211.497 & 164.197 & 214.195 & 33.323 & 86538 & 35 & 30 \\
\hline
\end{tabular}

\begin{tabular}{c|r|r|r|r} 
Version & Standard & Elkhound & $\begin{array}{r}\text { Incremental } \\
\text { (no cache) }\end{array}$ & Incremental \\
\hline Average & 216.004 & 178.237 & 281.788 & 36.896 \\
\hline 0 & 217.891 & 171.354 & 281.202 & 267.256 \\
1 & 200.142 & 175.458 & 295.214 & 27.340 \\
2 & 217.900 & 176.373 & 275.369 & 29.527 \\
3 & 218.666 & 184.393 & 274.712 & 49.673 \\
4 & 220.651 & 180.516 & 295.892 & 51.704 \\
5 & 217.303 & 178.121 & 295.963 & 41.833 \\
6 & 210.368 & 179.741 & 280.381 & 32.282 \\
7 & 209.642 & 178.047 & 275.611 & 44.323 \\
8 & 220.227 & 177.271 & 279.323 & 49.866 \\
9 & 219.935 & 178.604 & 277.716 & 29.116 \\
10 & 222.944 & 172.873 & 270.160 & 40.494 \\
11 & 216.337 & 179.912 & 269.427 & 29.112 \\
12 & 217.212 & 176.450 & 293.319 & 31.444 \\
13 & 222.033 & 177.199 & 270.401 & 27.974 \\
14 & 212.739 & 177.661 & 280.448 & 28.142 \\
15 & 213.956 & 180.937 & 292.892 & 40.610
\end{tabular}




\section{Appendix B}

\section{SPLASH Conference 2019 - ACM Student Research Competition}

In the week of 20-25 October 2019, I had the opportunity to present my work at the ACM Student Research Competition (SRC), during the SPLASH conference in Athens, Greece. ${ }^{1}$ In the SRC held at SPLASH, I achieved first place in the category of graduate (Master and $\mathrm{PhD}$ ) students. The competition consisted of several rounds, with several deliverables, which have been listed below.

Call for Submissions: Extended Abstract To participate in the SRC, participants had to submit an extended abstract of no more than 800 words and no more than 2 pages (excluding references). Appendix B.1 contains my submission, which is also published in the Proceedings Companion of SPLASH 2019. ${ }^{2}$ After review, eight participants were invited to present their work at the conference, of which four undergraduate and four graduate students.

First Round: Poster On Wednesday the 23rd, the eight participants presented a poster showing their work to the jury. The poster that I presented is included in Appendix B.2 and available online on the website of the Programming Languages research group. ${ }^{3}$ The jury selected six participants (three in each category) to advance to the next round.

Second Round: Presentation On Thursday the 24th, the six selected participants gave a presentation of ten minutes followed by a question session of five minutes. In each category, the winning participant advanced to the Grand Finals.

Grand Finals: Short Paper The winners of all SRCs throughout the year competed in the Grand Finals. A different panel of judges evaluated these winners against each other via the web. Three undergraduates and three graduates were chosen as the SRC Grand Finals winners. Finalists had to submit a short paper of no more than 4000 words and no more than 5 pages (excluding references). Appendix B.3 contains my submission, which is also available on the website of the ACM SRC. ${ }^{4}$ It was not selected as one of the winners but did receive one "weak accept" and two "accept" judgements.

\footnotetext{
${ }^{1}$ https://2019.splashcon.org/track/splash-2019-SRC

${ }^{2}$ https://dl.acm.org/doi/10.1145/3359061.3361085

${ }^{3}$ https://pl.ewi .tudelft.nl/posters/2019/10/22/incremental-scannerless-generalized-lr-parsing/

${ }^{4}$ https://src.acm.org/binaries/content/assets/src/2020/maarten-p.-sijm.pdf
} 


\title{
Incremental Scannerless Generalized LR Parsing
}

\author{
Maarten P. Sijm \\ Delft University of Technology \\ Delft, The Netherlands \\ mpsijm@acm.org
}

\begin{abstract}
We present the Incremental Scannerless Generalized LR (ISGLR) parsing algorithm, which combines the benefits of Incremental Generalized LR (IGLR) parsing and Scannerless Generalized LR (SGLR) parsing. The parser preprocesses the input by modifying the previously saved parse forest. This allows the input to the parser to be a stream of parse nodes, instead of a stream of characters. Scannerless parsing relies heavily on non-determinism during parsing, negatively impacting the incrementality of ISGLR parsing. We evaluated the ISGLR parsing algorithm using file histories from Git, achieving a speedup of up to 25 times over non-incremental SGLR.
\end{abstract}

CCS Concepts • Software and its engineering $\rightarrow$ Incremental compilers; Parsers.

Keywords incremental, scannerless, GLR, IGLR, SGLR, ISGLR, parsing, Spoofax

\section{ACM Reference Format:}

Maarten P. Sijm. 2019. Incremental Scannerless Generalized LR Parsing. In Proceedings of the 2019 ACM SIGPLAN International Conference on Systems, Programming, Languages, and Applications: Software for Humanity (SPLASH Companion '19), October 20-25, 2019, Athens, Greece. ACM, New York, NY, USA, 3 pages. https: //doi.org/10.1145/3359061.3361085

\section{Background}

Visser introduced Scannerless Generalized LR (SGLR) parsing, which combines the lexical and context-free phases of Generalized LR (GLR) parsing. [5] The terminals in the grammar are single characters instead of tokens. This has several advantages: it removes the need for a separate lexing (or scanning) phase, supports modelling the entire language syntax in one single grammar, and composing grammars for

Permission to make digital or hard copies of all or part of this work for personal or classroom use is granted without fee provided that copies are not made or distributed for profit or commercial advantage and that copies bear this notice and the full citation on the first page. Copyrights for components of this work owned by others than the author(s) must be honored. Abstracting with credit is permitted. To copy otherwise, or republish, to post on servers or to redistribute to lists, requires prior specific permission and/or a fee. Request permissions from permissions@acm.org. SPLASH Companion '19, October 20-25, 2019, Athens, Greece

(C) 2019 Copyright held by the owner/author(s). Publication rights licensed to ACM.

ACM ISBN 978-1-4503-6992-3/19/10 . .\$15.00

https://doi.org/10.1145/3359061.3361085 different languages. One notable disadvantage is that the SGLR parsing algorithm is a batch algorithm: it processes each input file in its entirety. This becomes a problem for software projects that have large files, as every small change requires the entire file to be parsed again.

Wagner [6] and TreeSitter [4], amongst others, have introduced Incremental Generalized LR (IGLR) parsing algorithms that improve upon batch GLR parsing by incrementally parsing changes to large files. However, these algorithms use a separate incremental lexical analysis phase which complicates the implementation of incremental parsing and does not directly allow language composition.

\section{Incremental Scannerless GLR Parsing}

We present the Incremental Scannerless Generalized LR (ISGLR) parsing algorithm, which combines the benefits of IGLR parsing and SGLR parsing. We implemented the algorithm as part of the Spoofax language workbench [2] as a modular extension to the Java implementation of SGLR (JSGLR2). [1] We will discuss the main ideas of our parsing algorithm.

Input Preprocessing After successfully parsing an input file, the parser saves both the input string and the resulting parse forest. When reparsing the same file, it calculates the difference between the previous and the new input strings. The changes can be deletions or insertions, or both at the same time. From the previous parse forest, the parser removes parse nodes that fall within a deleted region and creates a new (temporary) parse node for every inserted region, which contains the inserted characters as children. Changed parse nodes will no longer be valid, which will be fixed during parsing.

Parsing Instead of a stream of characters, the input to the parsing algorithm is a stream of parse nodes. These parse nodes can either be internal nodes (corresponding to grammar productions) or terminal nodes (corresponding to characters).

When parsing starts, the input stream consists only of the pre-processed parse forest and the end-of-file marker. When the parser encounters an invalid parse node in the input stream, it is broken down, meaning that its child nodes will become part of the input stream instead. Ultimately, the parser will break down all parse nodes on the spines from the root to the changed regions. An example of this is shown in Figure 1. 

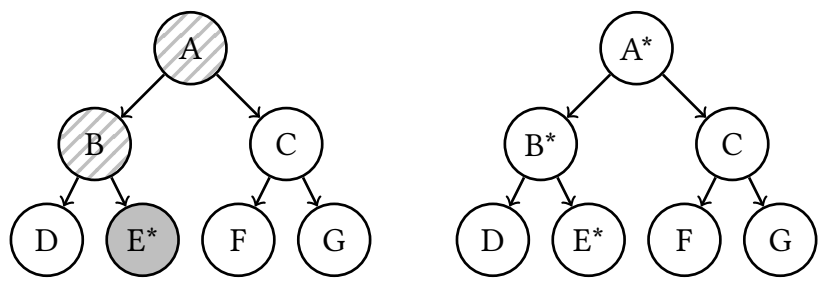

(a) Left: a preprocessed parse tree, where node $\mathrm{E}$ has been changed. Because of this, its ancestors A and B become invalid. Right: the resulting parse tree after reparsing.

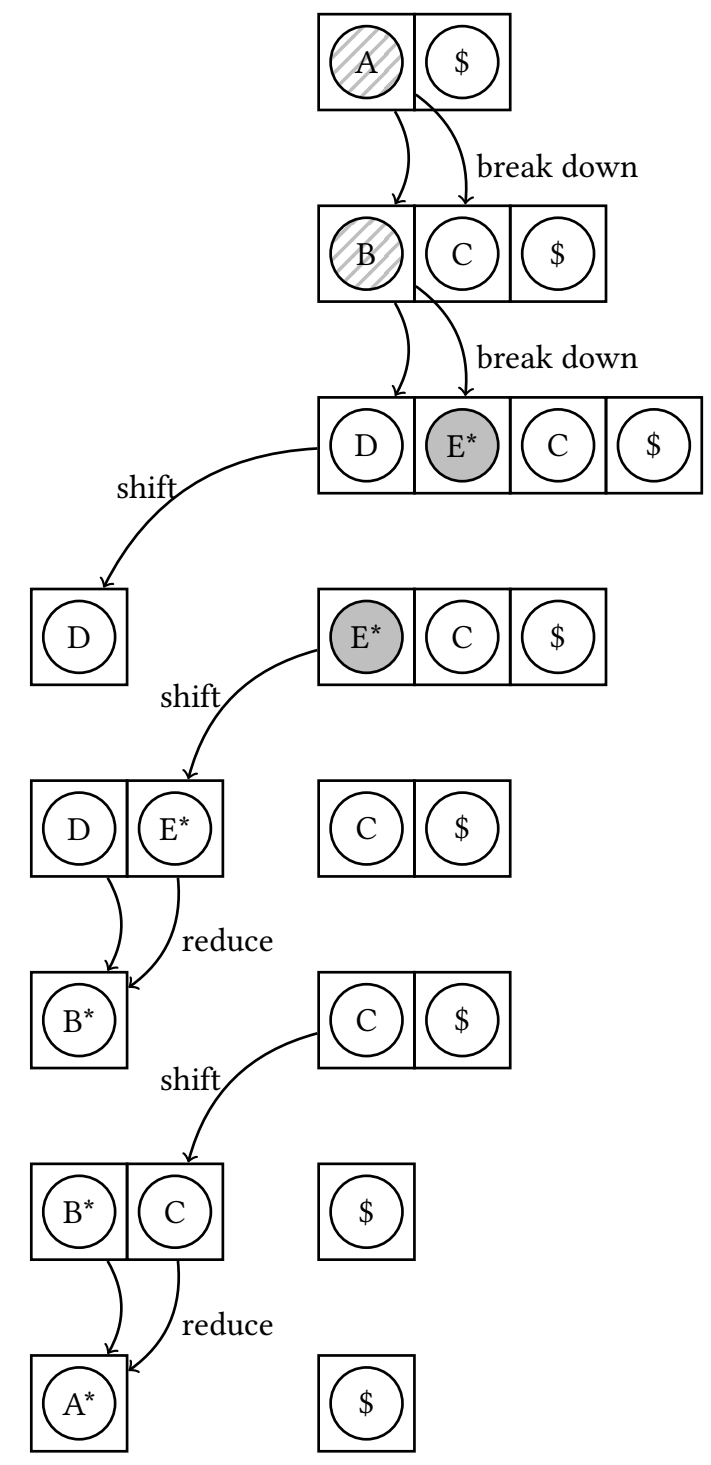

(b) The parse stack is on the left and the input stream is on the right. During parsing, the invalid nodes A and B are broken down. Node D and subtree C can be fully reused.

Figure 1. An example of how the input stream and parse stack are behaving during incremental parsing.
State Matching A state matching test decides whether an unchanged internal node from the input stream can be reused or not. The parser will store in all parse nodes the top-most state of the parse stack that it was pushed onto. If the current state of the parser is equal to the state stored in the next node of the input stream, it can be reused; else, it must be broken down.

Non-determinism When there are multiple possible actions, GLR parsers will split into multiple stacks and run the parsing algorithm concurrently on these stacks, synchronizing on shift actions. [3] Any stacks that have no applicable actions are discarded. As long as there are no ambiguities in the grammar, only one parse stack will remain. With a reparse, a change right after a non-deterministic region can cause a different parse stack to survive. As a result, any parse node that was created during non-deterministic parsing must be broken down.

SGLR parsing relies heavily on the fact that the parser is non-deterministic because character-level grammars frequently need arbitrary length lookahead. [5] Unfortunately, this means that the number of parse nodes that can be reused is a lot less than for IGLR parsing. It is not yet clear how to reduce non-determinism in character-level grammars.

\section{Evaluation}

We evaluated the ISGLR parsing algorithm with Git repositories, using the file differences between commits as input to the parser. Preliminary results show that the incremental parser is on average 13\% slower than the JSGLR2 parser when parsing a file from scratch, but achieves a speed-up when parsing the files incrementally. The speedup of ISGLR over JSGLR2 ranges from $15 \%$ faster (for parsing all versions of all files in a repository ${ }^{1}$ ) to 25 times faster (for a single file of 90 kilobytes that has changes averaging 700 bytes $^{2}$ ).

\section{Conclusion}

Our main contribution is the ISGLR algorithm, which combines SGLR parsing with IGLR parsing. An open challenge for this algorithm is that typically fewer parse nodes can be reused than with IGLR parsing. However, in typical use cases, the ISGLR parsing algorithm will still perform better than the non-incremental variant.

\section{References}

[1] Jasper Denkers. 2018. A Modular SGLR Parsing Architecture for Systematic Performance Optimization. Master's thesis. Delft University of Technology, Delft, The Netherlands. Advisor(s) Eelco Visser, Michael Steindorfer, Eduardo de Souza Amorim. http://resolver.tudelft.nl/uuid: 7d9f9bcc-117c-4617-860a-4e3e0bbc8988

$\overline{{ }^{1} \text { https://github.com/metaborg/mb-rep/tree/ }}$ e33de52a766a1df6cbef79f069c3ebab822ef6e0

${ }^{2}$ https://github.com/AnySoftKeyboard/AnySoftKeyboard/blob/ 16570810a492188687ad074679c74a9114291aa2/app/src/main/java/ com/anysoftkeyboard/keyboards/views/AnyKeyboardViewBase.java 
[2] Lennart C.L. Kats and Eelco Visser. 2010. The Spoofax Language Workbench: Rules for Declarative Specification of Languages and IDEs. In Proceedings of the ACM International Conference on Object Oriented Programming Systems Languages and Applications (OOPSLA '10). ACM, New York, NY, USA, 444-463. https://doi.org/10.1145/1869459.1869497

[3] Jan G Rekers. 1992. Parser generation for interactive environments. Ph.D. Dissertation. University of Amsterdam.
[4] TreeSitter. 2019. TreeSitter Documentation. Retrieved May 23, 2019 from http://tree-sitter.github.io

[5] Eelco Visser et al. 1997. Scannerless generalized-LR parsing. Universiteit van Amsterdam. Programming Research Group.

[6] Tim A Wagner. 1997. Practical algorithms for incremental software development environments. Ph.D. Dissertation. University of California, Berkeley. 


\section{Incremental Scannerless Generalized LR Parsing}

Maarten P. Sijm*

*Delft University of Technology, Programming Languages group mpsijm@acm.org
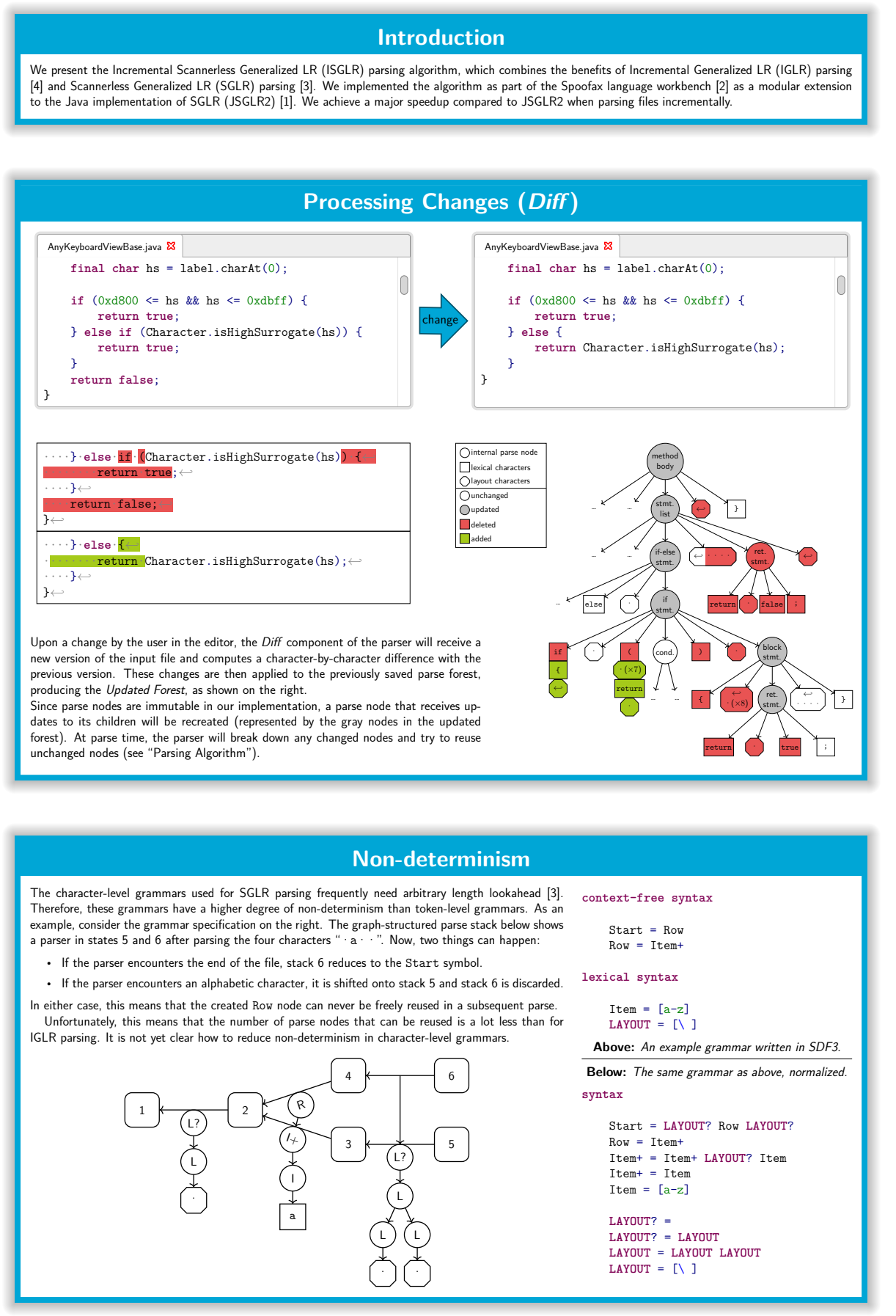

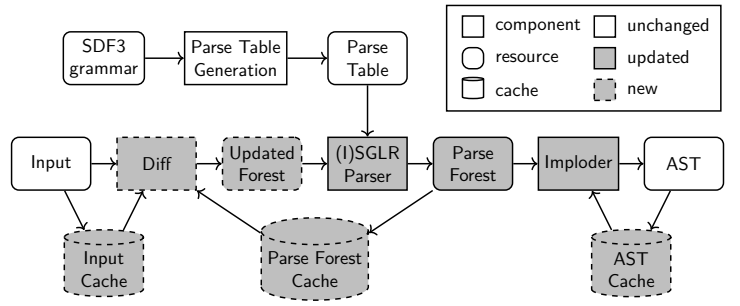

The incremental parsing pipeline architecture.

Top row: executed during language development.

Middle row: executed every time that a file is parsed.

Bottom row: caches that are maintained between parses.
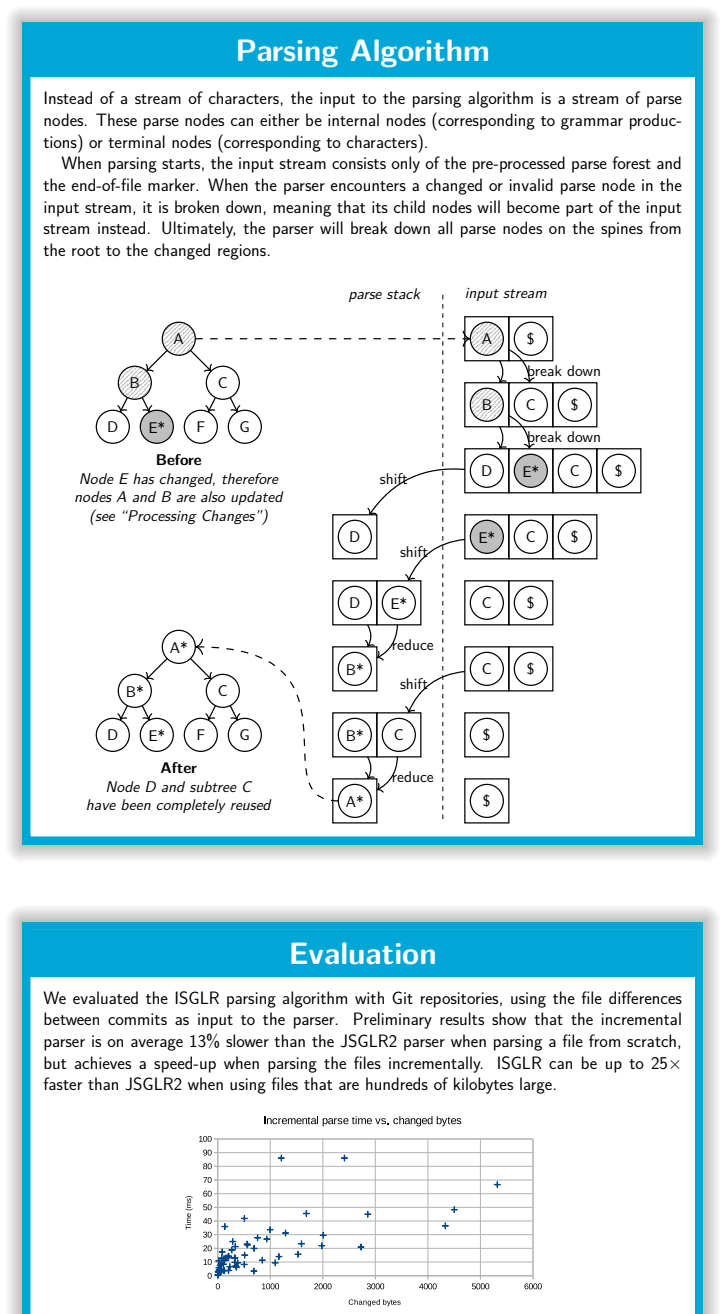

The same incremental parsing times as in the plot below, showing the relation between change sizes and incremental parsing time.
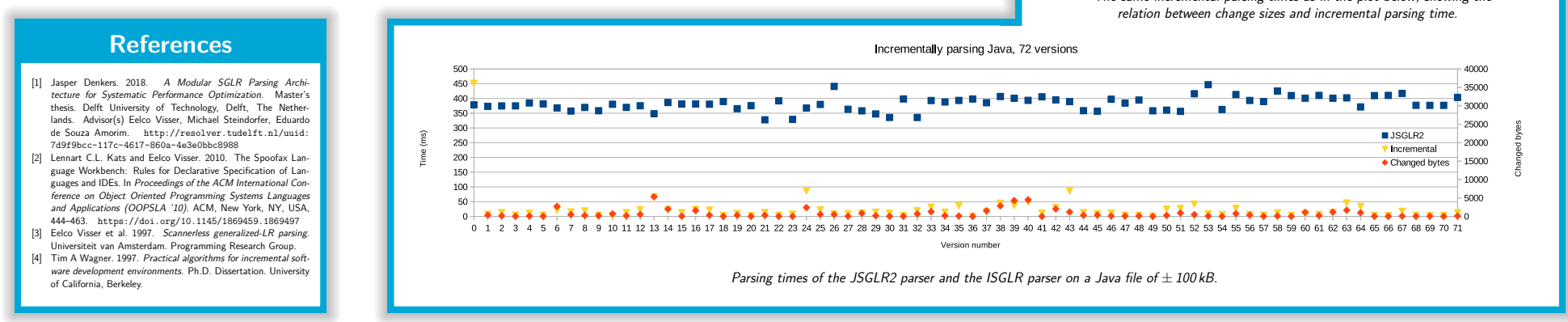


\title{
SPLASH: G: Incremental Scannerless Generalized LR Parsing
}

\author{
Maarten P. Sijm \\ Delft University of Technology \\ Delft, The Netherlands \\ mpsijm@acm.org
}

\begin{abstract}
We present the Incremental Scannerless Generalized LR (ISGLR) parsing algorithm, which combines the benefits of Incremental Generalized LR (IGLR) parsing and Scannerless Generalized LR (SGLR) parsing. The ISGLR parser can reuse parse trees from unchanged regions in the input and thus only needs to parse changed regions. We also present incremental techniques for imploding the parse tree to an Abstract Syntax Tree (AST) and syntax highlighting. Scannerless parsing relies heavily on non-determinism during parsing, negatively impacting the incrementality of ISGLR parsing. We evaluated the ISGLR parsing algorithm using file histories from Git, achieving a speedup of up to 25 times over non-incremental SGLR.
\end{abstract}

CCS Concepts • Software and its engineering $\rightarrow$ Incremental compilers; Parsers.

Keywords incremental, scannerless, parsing, GLR, IGLR, SGLR, ISGLR, imploding, syntax, Spoofax

\section{ACM Reference Format:}

Maarten P. Sijm. 2020. SPLASH: G: Incremental Scannerless Generalized LR Parsing. In ACM Student Research Competition Grand Finals, 2020. ACM, New York, NY, USA, 5 pages.

\section{Introduction}

Background Scannerless Generalized LR (SGLR) parsing combines the lexical and context-free phases of parsing. The terminals in the grammar are single characters instead of tokens. This has several advantages: it removes the need for

Permission to make digital or hard copies of all or part of this work for personal or classroom use is granted without fee provided that copies are not made or distributed for profit or commercial advantage and that copies bear this notice and the full citation on the first page. Copyrights for components of this work owned by others than the author(s) must be honored. Abstracting with credit is permitted. To copy otherwise, or republish, to post on servers or to redistribute to lists, requires prior specific permission and/or a fee. Request permissions from permissions@acm.org. ACM Student Research Competition, Grand Finals, 2020

(C) 2020 Copyright held by the owner/author(s). Publication rights licensed to ACM. a separate lexing (or scanning) phase, supports modelling the entire language syntax in one single grammar, and allows composition of grammars for different languages. One notable disadvantage is that the SGLR parsing algorithm of Visser [9] is a batch algorithm, meaning that it must process each entire file in one pass. This becomes a problem for software projects that have large files, as every small change requires the entire file to be parsed again.

Incremental Generalized LR (IGLR) parsing is an improvement over batch Generalized LR (GLR) parsing. Amongst others, Wagner [10] and TreeSitter [8] have created parsing algorithms that allow rapid parsing of changes to large files. However, these algorithms use a separate incremental lexical analysis phase which complicates the implementation of incremental parsing [11] and does not directly allow language composition.

Contributions In Section 2, we present the Incremental Scannerless Generalized LR (ISGLR) parsing algorithm that combines the benefits of incremental and scannerless GLR parsing. The algorithm only considers changed parts of the input and includes a test that prevents unchanged parse nodes from being reused incorrectly when a change in the context would require them to be parsed differently.

We explain the impact of non-determinism on the ISGLR parsing algorithm in Section 3. This effect explains that the combined algorithm cannot reuse as much from previous results as the non-scannerless IGLR parsing algorithm.

In Section 4, we discuss the integration of the ISGLR parsing algorithm in the Spoofax language workbench [3]. It is implemented as an extension to the Java implementation of SGLR (JSGLR2) [2]. Specifically, we focus on imploding to an Abstract Syntax Tree (AST) and on syntax highlighting.

We have evaluated the algorithm on input from the Git version control system, as shown in Section 5. Regardless of the non-determinism issue, the ISGLR parsing algorithm performs up to 25 times faster than batch parsing for small changes to large files. 


\section{Incremental Scannerless GLR Parsing}

We present the ISGLR parsing algorithm, which combines the benefits of IGLR parsing and SGLR parsing. We will discuss the main ideas of our parsing algorithm below.

Input Preprocessing The input to the ISGLR parser consists of two parts: a list of changes between the previous and the current input strings, and the parse tree that resulted from the previous parse. The changes can be deletions or insertions, and having both at the same time represents a replacement. From the previous parse tree, the parser removes parse nodes that fall within a deleted region and creates a new (temporary) parse node for every inserted region, which contains the inserted characters as children.

Parse nodes store the width of their subtree to make this preprocessing step efficient. The width corresponds to the number of characters of the input represented by the subtree. With the exact position of a change, the subtrees requiring changes can be found using a traversal from the root node and recursively picking the child that contains this position.

Any new parse nodes created in this process are marked as irreusable, signalling to the parser that they are not valid for reuse. This includes the parse nodes created because of insertions and replacements. In addition, all ancestors of any changed nodes are marked irreusable, simply because they have one or more descendants that cannot be reused, like the nodes $\mathrm{A}$ and $\mathrm{B}$ in the example of Figure $1 \mathrm{a}$.

Parsing Instead of a stream of characters, the input to the parsing algorithm is a stream of parse nodes. These parse nodes can either be internal nodes (corresponding to grammar productions) or terminal nodes (corresponding to characters).

When parsing starts, the input stream consists only of the preprocessed parse tree and the end-of-file marker. When the parser encounters an irreusable parse node in the input stream, it is broken down, meaning that its child nodes will become part of the input stream instead. Ultimately, the parser will break down all parse nodes on the spines from the root to the changed regions. An example of this is shown in Figure 1b.

State Matching The state matching test is an extra check to decide whether an unchanged internal node from the input stream can be reused or not. This prevents the parser from blindly reusing a parse node that needs to be parsed differently because a part of the input has changed before the current position.

To accomplish this, the parser will store in each parse node the topmost state of the parse stack that it was pushed onto, so that it can be used for the state matching test during a subsequent incremental parse. During parsing, if the current state of the parser is equal to the state stored in the next node of the input stream, this node can be reused; else, it must be broken down like other irreusable parse nodes.
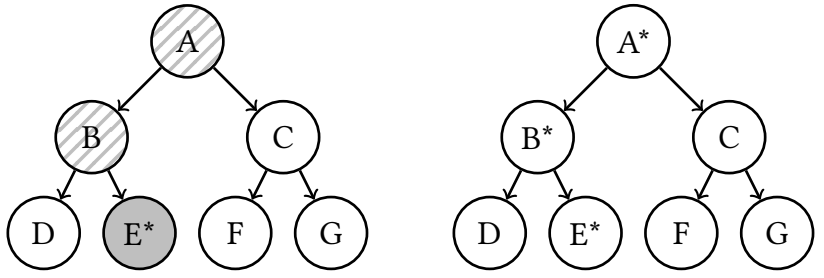

(a) Left: a preprocessed parse tree, where node $\mathrm{E}$ has been changed. Because of this, its ancestors A and B are irreusable.

Right: the resulting parse tree after reparsing.

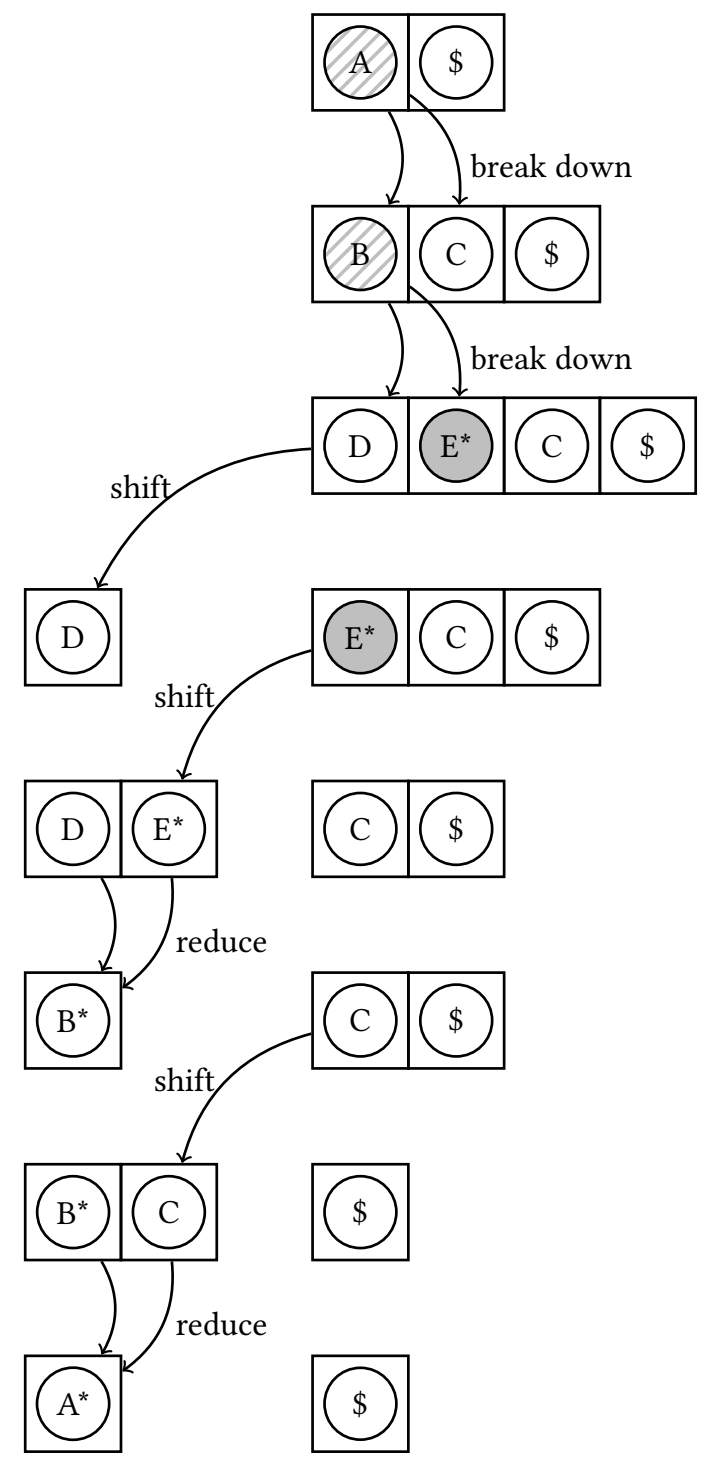

(b) The parse stack is on the left and the input stream is on the right. During parsing, the invalid nodes A and B are broken down. Node D and subtree $\mathrm{C}$ can be fully reused.

Figure 1. An example of how the input stream and parse stack are behaving during incremental parsing. 

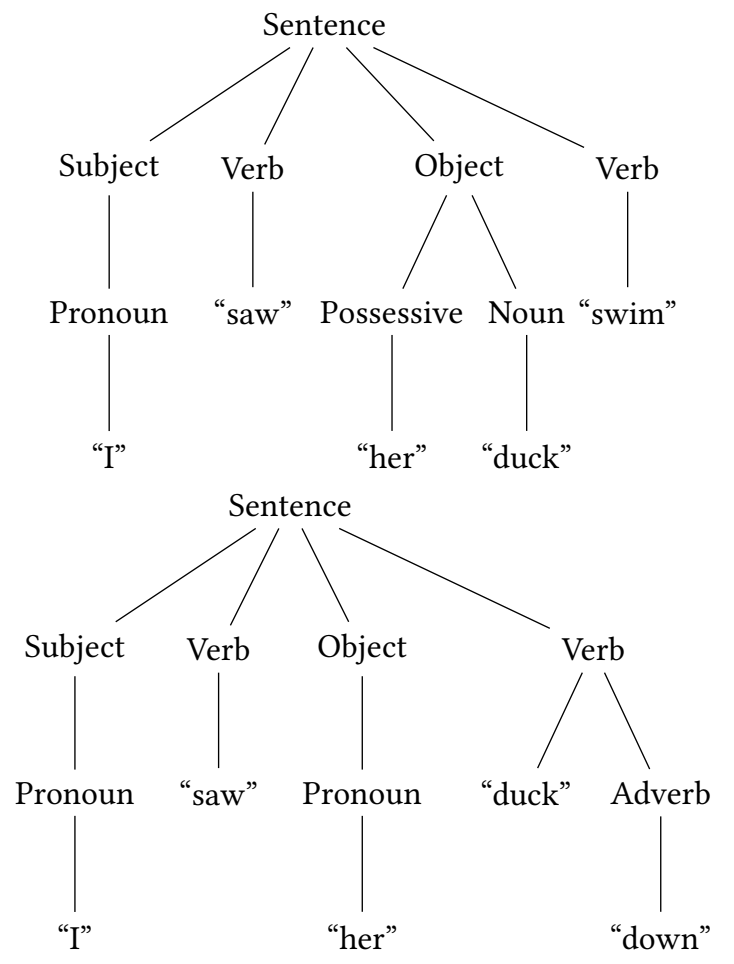

Figure 2. An example where non-determinism is used during parsing. In both sentences "I saw her duck swim" and "I saw her duck down", there is no ambiguity in the final result, but the word "her" has a different interpretation depending on the final word of the sentence. Therefore, the parser must explore both possibilities until it encounters the disambiguating final word.

\section{Non-determinism}

When a GLR parser reaches a point where multiple actions are possible, it will split the parse stack into multiple stacks and run the parsing algorithm concurrently on these stacks, synchronizing on shift actions [6]. Any stacks that have no applicable actions are discarded. As long as there are no ambiguities in the grammar, only one parse stack will remain.

Example To illustrate this, consider the example in Figure 2, showing two parse trees for two slightly different English sentences: "I saw her duck swim" and "I saw her duck down". Both sentences are not ambiguous in their meaning, but the words "her" and "duck" do have a different interpretation depending on the last word of the sentence. For the simplicity of this example, assume that these two sentences are the only possible valid sentences in English.

If these sentences were parsed using a GLR parser, the parser would split the parse stack when encountering the word "her". One parse stack would explore the possibility of "her" being a possessive pronoun, while the other would try to parse "her" as being a regular pronoun. This signals the start of a non-deterministic region in the input sentence: the parser can not directly know which interpretation is the correct one, so it explores all possibilities. Only when the parser reaches the final word of the sentence, the parser can discard the parse stack that has the incorrect interpretation and it continues with the single remaining parse stack, which ends the non-deterministic region in the input.

Impact on Incrementality Consider the previous example in an incremental setting, parsing one sentence after the other using an incremental parse. The changed word right after the non-deterministic region causes a different parse stack to survive. Even though the words "her" and "duck" have not changed, an incremental parser cannot blindly reuse the result of the previous parse.

As a result, any parse node that is created during nondeterministic parsing must be marked as irreusable, so that it will be broken down during the incremental parse even when it is unchanged. This forces the parser to explore all possible interpretations again. In the cases where, after reparsing, the parser chooses the same interpretation as before, it has effectively wasted some time reparsing that part.

Impact on Scannerless Parsing SGLR parsing relies heavily on the fact that the parser is non-deterministic because character-level grammars frequently need arbitrary length lookahead [9]. Unfortunately, this means that the number of parse nodes that ISGLR parsing can reuse is a lot less than for IGLR parsing. It is not yet clear how to reduce nondeterminism in character-level grammars.

According to our measurements, about one-third of all parse nodes are marked as irreusable when parsing Java source code. However, on average only $2 \%$ of all parse nodes (of both kinds) were broken down during the experiment of Figures 4 and 5 in Section 5. The reason for this is as follows: while some irreusable nodes are exposed along the spine between the root and the changed regions, the majority of the irreusable parse nodes are not exposed and therefore the parser also does not need to break them down.

\section{Editor Integration}

Code editors can use the incremental result of an ISGLR parser to incrementally calculate the results of type checking and compilation, amongst others. In this research project, we focus on imploding the parse tree to an AST and on syntax highlighting.

We implemented the ISGLR parser in the Spoofax language workbench [3]. In Spoofax, language designers can specify programming languages declaratively by describing their syntax, static and dynamic semantics, and transformations. Spoofax then generates an editing environment for the language, including syntax highlighting and error checking. Our incremental parser adds to other recent work that 
incrementalizes the Spoofax pipeline, such as an incremental type checker [1], an incremental build system [4], and incremental compilation [7].

We implemented the ISGLR parsing algorithm as an extension to the JSGLR2 parsing algorithm [2]. The modular nature of JSGLR2 helped to only extend those parts of the parser that required changes to allow incremental parsing, resulting in about 1000 added lines of Java code.

Imploding The parse tree that the parser generates contains many details about the input program that are not relevant for further processing. Examples include whitespace and literal keywords (like if or return). The keywords are redundant information because they are always the same for their corresponding grammar rule and whitespace is redundant because it only contributes to the layout of the program, not the meaning of it.

In Spoofax, imploding is the post-processing step after parsing that removes this redundant information from the parse tree, producing an Abstract Syntax Tree (AST) that can be used in further processing. The baseline algorithm works in a top-down fashion: after processing a parse node, it processes the children of this node recursively.

In the design of the incremental imploding algorithm, we make use of two things. Firstly, we know that the parser only changed a small part of the parse nodes. All new parse nodes are reachable from the root of the parse tree via other changed parse nodes. Put differently, if a parse node is not changed, we can be certain that all its descendants are also not changed. Secondly, imploding is an operation that happens locally on parse nodes: no information of the parent node or any of the child nodes is required to process the current parse node. Because of this, we can store the resulting AST for each imploded parse node.

The incremental imploding algorithm also processes a parse tree recursively from the top down, with one key difference from the baseline algorithm: when encountering a parse node that already has a resulting AST, we can directly reuse this result. This ensures that only the parse nodes that are changed by the incremental parser are processed.

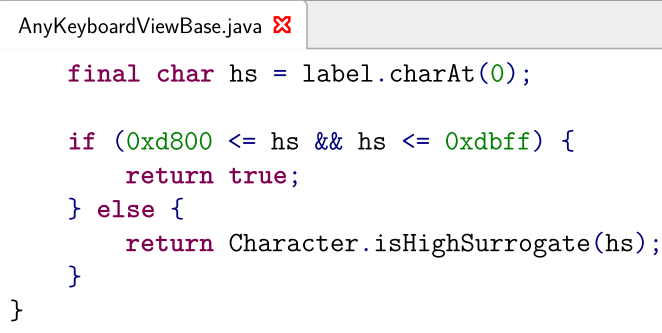

Figure 3. An editor showing syntax highlighting.

Syntax highlighting In most code editors, a program is displayed to the user using colours to give a visual indication about the different program elements in the code, as shown in Figure 3. This is also the case in Spoofax. Language designers can indicate which program elements get which colour and Spoofax will show the right colours in the editor.

Spoofax transforms the parse tree that resulted from the parser into a list of editor tokens, each given the correct colour based on the grammar rule that the parse node was created with. It might seem odd to create tokens when one of the features of scannerless parsing was that no tokens are required before parsing. However, there is one key difference with regular tokenization: for these tokens, information from the parser can be used to determine their type and colour. This means that the exact same word can be coloured differently based on context. Regular tokenizers like Lex only partially support this by allowing custom $C$ code to be executed and having a mechanism called start conditions [5]. However, this can never be as powerful as a full context-free parser, simply because then a parser would be unnecessary.

For the incremental syntax highlighter, we store the tokens as leaves to the AST and link them together to allow iterating over the tokens in linear time. Updating tokens in updated parts of the AST is done in a way similar to imploding: subtrees that did not change can be directly reused and changed subtrees require reprocessing. In the case of reprocessing, the links between tokens on the boundaries of the change must be updated to make sure that iterating over all tokens uses the most recent tokens.

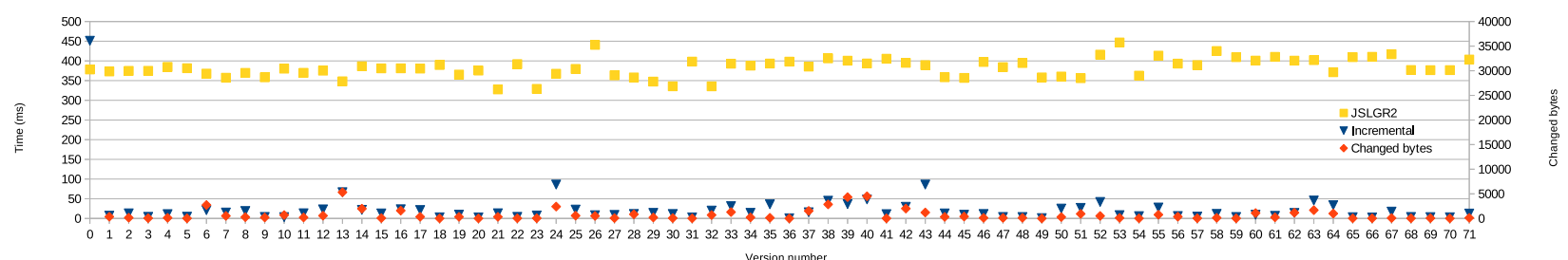

Figure 4. Parsing times of the JSGLR2 parser and the ISGLR parser on a Java file of almost $100 \mathrm{kB}$. The yellow squares and blue triangles indicate the parsing times (left y-axis) for these two parsers, respectively. The red diamonds indicate the number of changed bytes between each version (right y-axis). 


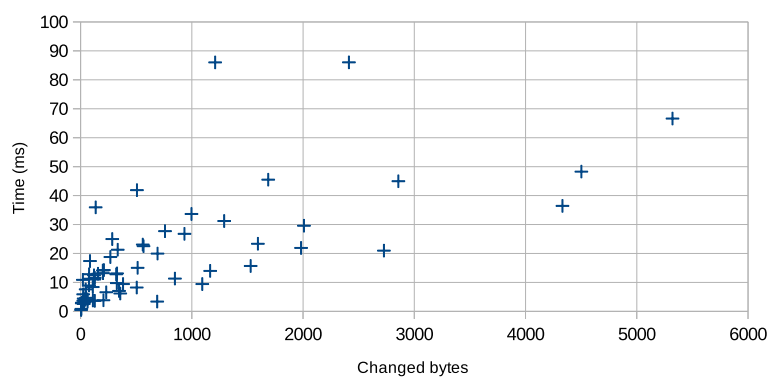

Figure 5. The same incremental parsing times as in Figure 4, showing the relation between change sizes and incremental parsing time.

\section{Evaluation}

We evaluated the runtime performance of the ISGLR parsing algorithm. As input to the parser, we use the file differences between commits in Git repositories. This experiment models how the parser would be used by a developer in their editor in the case that they would switch between commits in their local clone of the repository, for example, when they pull the latest changes committed by other developers. We are still working on experiments where user input is simulated as they would be working in the editor. The differences recorded in individual commits vary greatly in size and therefore cannot be directly used for this scenario.

It is important to distinguish between two types of results: those for batch parsing (where the full file is parsed from scratch) and for incremental parsing (where a new version of the file with a small change is parsed).

Preliminary results show that the ISGLR parser is on average 13\% slower than the JSGLR2 parser when performing a batch parse. This slight slowdown can be attributed to the need to store more data to perform incremental updates later.

However, for incremental parses, the ISGLR parser has a speedup over JSGLR2 ranging from 15\% faster (for parsing all versions of all files in a repository ${ }^{1}$ ) to 25 times faster (for a single file of 90 kilobytes that has changes averaging 700 bytes, with a standard deviation of 1100 bytes $^{2}$ ). The results for the last experiment are shown in Figures 4 and 5 . These figures show a slight correlation between the size of a change and the time needed by the ISGLR parser to perform an incremental parse on this change.

So far, our experiments have focused on evaluating just the parsing algorithm. In upcoming experiments, we will also evaluate the performance of the incremental imploding algorithm and incremental syntax highlighting.

\footnotetext{
${ }^{1}$ https : / / github . com / metaborg / mb - rep / tree / e33de52a766a1df6cbef79f069c3ebab822ef6e0

${ }^{2}$ https : / / github . com / AnySoftKeyboard / AnySoftKeyboard / blob / 16570810a492188687ad074679c74a9114291aa2/app/src/main/java/com/ anysoftkeyboard/keyboards/views/AnyKeyboardViewBase.java
}

\section{Conclusion}

Our main contribution is the ISGLR algorithm, which combines SGLR parsing with IGLR parsing. An open challenge for this algorithm is that typically fewer parse nodes can be reused than with IGLR parsing due to the non-deterministic nature of scannerless parsing. However, in typical use cases, the ISGLR parsing algorithm will still perform better than the non-incremental variant.

Within this research project, we implemented incremental algorithms for imploding the parse tree to an AST and for syntax highlighting. We are still in the process of evaluating the performance of these editor integration services.

\section{References}

[1] Taico Aerts. 2019. Incrementalizing Statix: A Modular and Incremental Approach for Type Checking and Name Binding using Scope Graphs. Master's thesis. Delft University of Technology, Delft, The Netherlands. Advisor(s) Eelco Visser, Hendrik van Antwerpen, Neil Yorke-Smith, Robbert Krebbers. http://resolver.tudelft.nl/uuid:3e0ea516-3058-4b8cbfb6-5e846c4bd982

[2] Jasper Denkers. 2018. A Modular SGLR Parsing Architecture for Systematic Performance Optimization. Master's thesis. Delft University of Technology, Delft, The Netherlands. Advisor(s) Eelco Visser, Michael Steindorfer, Eduardo de Souza Amorim. http://resolver.tudelft.nl/uuid: 7d9f9bcc-117c-4617-860a-4e3e0bbc8988

[3] Lennart C.L. Kats and Eelco Visser. 2010. The Spoofax Language Workbench: Rules for Declarative Specification of Languages and IDEs. In Proceedings of the ACM International Conference on Object Oriented Programming Systems Languages and Applications (OOPSLA '10). ACM, New York, NY, USA, 444-463. https://doi.org/10.1145/1869459.1869497

[4] Gabriël Konat, Michael J Steindorfer, Sebastian Erdweg, and Eelco Visser. 2018. PIE: A Domain-Specific Language for Interactive Software Development Pipelines. Art, Science and Engineering of Programming 2, 3 (2018), 1-31. https : / / doi . org / 10 . 22152 / programming journal.org/2018/2/9

[5] M. E. Lesk. 1975. Lex-A Lexical Analyzer generator. Technical Report CS-39. AT\&T Bell Laboratories, Murray Hill, N.J.

[6] Jan G Rekers. 1992. Parser generation for interactive environments. Ph.D. Dissertation. University of Amsterdam.

[7] Jeff Smits, Gabriël DP Konat, and Eelco Visser. 2020. Constructing Hybrid Incremental Compilers for Cross-Module Extensibility with an Internal Build System. The Art, Science, and Engineering of Programming 4, 3 (2020), 16-1.

[8] TreeSitter. 2019. TreeSitter Documentation. Retrieved May 23, 2019 from http://tree-sitter.github.io

[9] Eelco Visser et al. 1997. Scannerless generalized-LR parsing. Universiteit van Amsterdam. Programming Research Group.

[10] Tim A Wagner. 1997. Practical algorithms for incremental software development environments. Ph.D. Dissertation. University of California, Berkeley.

[11] Tim A. Wagner and Susan L. Graham. 1997. General Incremental Lexical Analysis. (1997). 


\section{Appendix C}

\section{Spoofax Enhancements}

The Spoofax language workbench simplifies the development of programming languages by generating many of the common features for a language based on declarative specifications. During my thesis, I have worked on several enhancements of Spoofax that were not directly related to my research on incremental parsing, but rather served as "side quests". Some of these enhancements were significant enough that I did not want to leave them unmentioned.

Unicode Support Before this enhancement, languages created in Spoofax were constrained to use characters in the ASCII range $([\backslash 0-\backslash 255])$. The EOF value was hardcoded to have value $\backslash 256$, which hindered the full adoption of Unicode in JSGLR and SDF. In a series of pull requests, ${ }^{1}$ I refactored the EOF value to be -1 instead and adapted the parser (generator) code to work with characters wider than 16 bits. The parse table generator now explicitly handles the EOF as a separate entity, while before, it was just appended to the end of the character class ranges. In addition, SDF3 now supports binary (e.g., \@b101010), octal (e.g., $\backslash 052$ ), and hexadecimal (e.g., $\backslash 0 \times 2 a$ ) escape codes in character classes, to aid in specifying Unicode characters with high code point values. An example of a Spoofax language that uses Unicode characters is shown in Figure C.1.

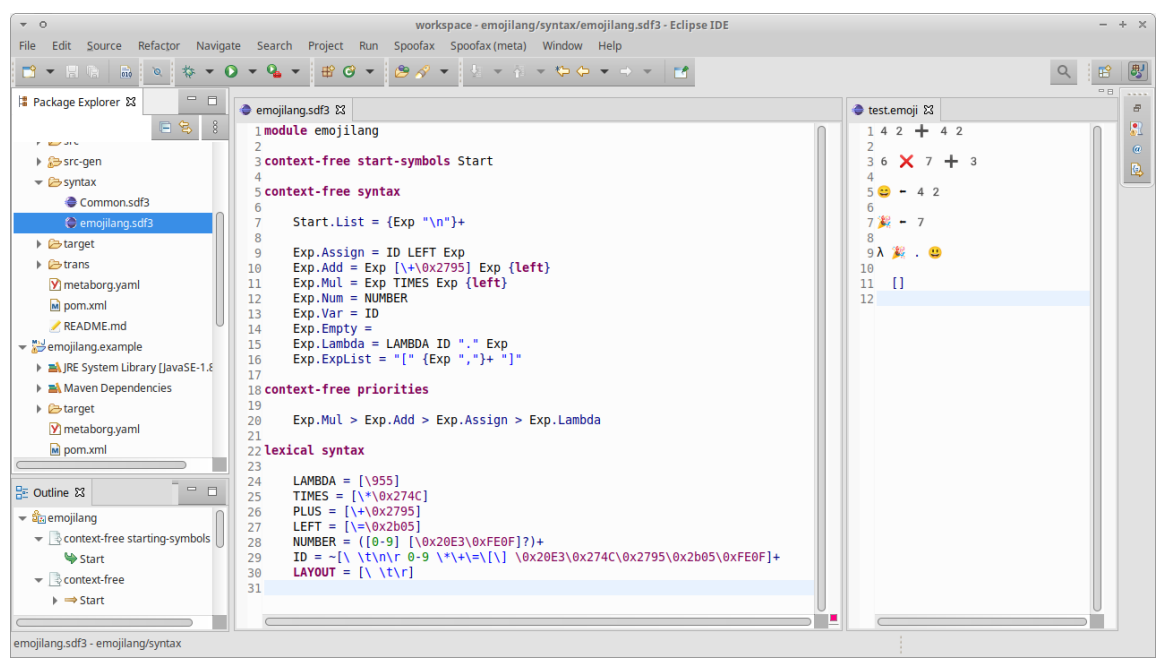

Figure C.1: A screenshot of Spoofax in Eclipse, showing a language that uses Unicode characters. The language supports the lambda symbol, emoji for addition and multiplication symbols and the left-pointing arrow, and numbers with combining code points that turn them into emoji. In this language, identifiers can consist of any characters that are not already used in the language.

\footnotetext{
${ }^{1}$ https://github.com/metaborg/jsglr/pull/72, https://github.com/metaborg/sdf/pull/38, and more
} 
Spoofax Syntax Highlighting in LaTeX Typesetting larger snippets of code in LaTeX with syntax highlighting is usually done with the listings or minted package. However, these packages typically only support the most-used General-Purpose Languages (GPLs). Using them to typeset a Domain-Specific Language (DSL) generally involves a bunch of workarounds, including manually listing keywords or manually overriding colours for certain parts of the displayed code. Therefore, Chiel Bruin and I have automated the typesetting of languages that are built with Spoofax. ${ }^{2}$ The implementation is a plugin for the Python package pygments, which is used by the minted package to generate syntax highlighting in LaTeX. Our plugin wraps the JSGLR2 command-line JAR ${ }^{3}$ and converts the resulting tokens and associated colours to a format that pygments understands. This thesis also makes use of this tool to typeset code snippets of SDF3, a DSL that is also defined in Spoofax, for example in Figures 2.7 and 3.1.

Spoofax Dark Theme Many text editors for programming languages (and many other applications as well) include a dark theme, to reduce eye strain from looking at a bright screen for a long period. The Spoofax language workbench is implemented as a plugin in Eclipse and IntelliJ, two major IDEs that have both light and dark themes. However, the languages generated by Spoofax are fixed to a single colouring scheme, defined in the ESV metalanguage. ${ }^{4}$ This colouring scheme is usually defined to have dark letters on a light background, so setting Eclipse to use a dark theme makes Spoofax languages unreadable. To resolve this, I devised a workaround (also known as "hack") that inverts the lightness of the colours defined in ESV when Eclipse is using a dark theme, ${ }^{5}$ which can be used until ESV changes the way it uses colour schemes. I preferred this over simply inverting the colours since that results in very bright text colours for most Spoofax languages. An example of a Spoofax instance in Eclipse with a dark theme is shown in Figure C.2.

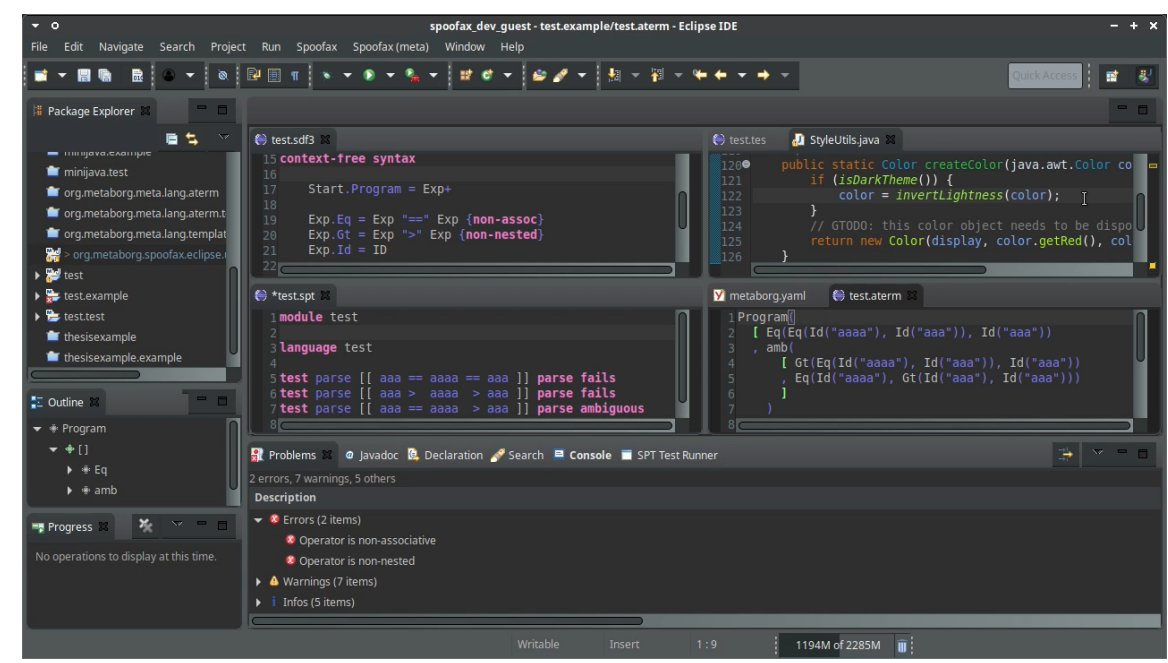

Figure C.2: A screenshot of Spoofax in Eclipse, using a dark theme. Top-left: SDF3. Top-right: Java (default Eclipse colours, not the Spoofax colours). Bottom-left: the Spoofax testing language (SPT). Bottom-right: the ATerm language, which describes ASTs.

\footnotetext{
${ }^{2}$ https://github.com/ChielBruin/spoofax-latex-tools/\#spoofax-pygments

${ }^{3}$ https://github.com/metaborg/jsglr/tree/master/org.spoofax.jsglr2.cli

${ }^{4}$ https://www. metaborg.org/en/latest/source/langdev/meta/lang/esv.html\#syntax-highlighting

${ }^{5}$ https://github.com/metaborg/spoofax-eclipse/pull/19
} 
SLR(1) Parse Table Generation As an unfinished experiment, I have implemented an SLR(1) parse table generator in SDF3. ${ }^{6}$ While SLR(1) parse table generation works for most grammars, it currently does not yet work for layout-sensitive grammars. In addition, it still needs a configuration option in the properties file of Spoofax languages (metaborg.yaml). I have used this experimental branch to test if it improves the performance of the ISGLR parser, but its impact did not look promising enough to finish the implementation. However, it did help to provide me insight on the different parsing behaviour between $\operatorname{LR}(0)$ and $(S) \operatorname{LR}(1)$ parse tables, which I refer to in Sections 3.2 and 3.3.

${ }^{6}$ https://github.com/metaborg/sdf/pull/27 